Simulação numérica de equações de conservação usando esquemas "upwind"

Juliana Bertoco 

Data de Depósito:

Assinatura:

\section{Simulação numérica de equações de conservação usando esquemas "upwind"}

\section{Juliana Bertoco}

Orientador: Prof. Dr. Valdemir Garcia Ferreira

Dissertação apresentada ao Instituto de Ciências Matemáticas e de Computação - ICMC-USP, como parte dos requisitos para obtenção do título de Mestre em Ciências - Ciências de Computação e Matemática Computacional. VERSÃO REVISADA

USP - São Carlos

Junho de 2012 
Ficha catalográfica elaborada pela Biblioteca Prof. Achille Bassi e Seção Técnica de Informática, ICMC/USP, com os dados fornecidos pelo(a) autor(a)

\begin{tabular}{|c|c|}
\hline \multirow[t]{3}{*}{ B482s } & $\begin{array}{l}\text { Bertoco, Juliana } \\
\quad \text { Simulação numérica de equações de conservação usandd } \\
\text { esquemas \"upwind " / Juliana Bertoco; orientador } \\
\text { Valdemir Garcia Ferreira. -- São Carlos, } 2012 \text {. } \\
\quad 139 \text { p. }\end{array}$ \\
\hline & $\begin{array}{l}\text { Dissertação (Mestrado - Programa de Pós-Graduação en } \\
\text { Ciências de Computação e Matemática Computacional) -- } \\
\text { Instituto de Ciências Matemáticas e de Computação, } \\
\text { Universidade de São Paulo, } 2012 \text {. }\end{array}$ \\
\hline & $\begin{array}{l}\text { 1. Mecânica dos fluidos. I. Ferreira, Valdemir } \\
\text { Garcia, orient. II. Título. }\end{array}$ \\
\hline
\end{tabular}


"É preciso deixar marcas de eterno por onde passamos e com quem convivemos". - Pe. LÉO 


\section{Agradecimentos}

Primeiramente, agradeço à Deus, meu criador e amigo, pelo dom de minha vida e por Sua presença à iluminar meu caminho ao longo deste trabalho.

Aos meus queridos pais, Oscar e Maria Marta, pelas orações, paciêcia, compreensão e pelo grande apoio ao longo de meus estudos. A minha irmã Joice, ao meu irmão Danilo e aos meus cunhados Mauro e Edivânia por todo carinho, apoio, torcida. E ainda, de maneira especial, aos meus sobrinhos Lara, Heitor e Eloá por todos os momentos de risos e sapequices que me transbordam de alegria.

Ao meu orientador Prof. Dr. Valdemir Garcia Ferreira pela disponibilidade, paciência, apoio e principalmente por seus ensinamentos.

À minha amiga Gabriela Aparecida dos Reis por sua amizade, paciência, carinho, apoio e por todos os bons momentos.

Aos amigos do LMACC-USP por toda a ajuda, ensinamentos, paciência e trabalho em equipe.

Aos meus irmãos de GOU, por todas as orações e pelos intensos momentos de fé e alegria.

Aos funcionários do ICMC-USP por toda dedicação e apoio, em especial à Leonardo Martinussi pela prontidão e paciência.

À todos os professores do ICMC-USP pelos ensinamentos e disponibilidade ao longo de todos estes anos de estudo.

À CAPES pelo suporte financeiro no desenvolvimento deste projeto de pesquisa.

Enfim, à todos que direta ou indiretamente contribuíram para a realização deste trabalho. 


\section{Resumo}

Uma família de esquemas upwind denominada FUS-RF (Family of Upwind Scheme via Rational Functions), que é derivada via funções racionais e dependentes de parâmetros, é proposta para o cálculo de soluções aproximadas de equações de conservação. A fim de ilustrar a capacidade dos novos esquemas, vários resultados computacionais para sistemas hiperbólicos de leis de conservação são apresentados. Esses testes mostram a influência dos parâmetros escolhidos sobre a qualidade dos resultados numéricos. Fazendo o uso de alguns testes padrões, comparação dos novos limitadores de fluxo correspondentes com o esquema bem estabelecido van Albada e esquema atual EPUS (Eight-degree Polynomial Upwind Scheme) é também realizada. Os testes numéricos realizados em transporte de escalares e problemas de dinâmica dos gases confirmam que alguns esquemas da família FUS-RF são não oscilatórios e fornecem resultados confiáveis quando perfis descontínuos são transportados. Um esquema particular dessa nova família de esquemas upwind é então selecionado e utilizado para resolver escoamentos complexos com superfícies livres móveis.

Palavras-chave: Equações de conservação; fluidodinâmica computacional; EDPs predominantemente convectivas; esquemas upwind. 


\section{Abstract}

A family of upwind schemes named as FUS-RF (Family of Upwind Scheme via Rational Functions), which is derived via rational functions and dependent of parameters, is proposed for computing approximated solutions of conservation equations. In order to illustrate the capability of the new schemes, several computational results for system of hyperbolic conservation laws are presented. These results clarify the influence of the chosen parameters on the quality of the numerical calculations. Using some standard test cases, comparison of the new corresponding limiters with the well established van Albada and the recently introduced EPUS (Eight-degree Polynomial Upwind Scheme) limiters is also done. Numerical tests on both scalar and gas dynamics problems confirm that some schemes of the FUS-RF family are non-oscillatory and yield sharp results when solving profiles with discontinuities. A particular upwind scheme of this new family is then selected and used for solving complex incompressible moving free surface flows.

key-words: Conservation equations; computational fluid dynamics; PDEs predominantly convective; upwind schemes. 



\section{Sumário}

1 Introdução 1

1.1 Motivação e objetivos . . . . . . . . . . . . . . . . . 2

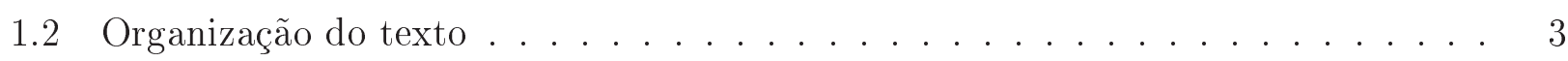

2 Formulação matemática $\quad 5$

2.1 Equações de conservação hiperbólicas . . . . . . . . . . . . . . . 5

2.1 .1 Equação linear de advecção . . . . . . . . . . . . . . . . . 5 5

2.1.2 Equação não linear de Burgers . . . . . . . . . . . . . . . . 6

2.1 .3 Equações de águas rasas . . . . . . . . . . . . . . . . . . 6

2.1.4 Equações de Euler . . . . . . . . . . . . . . . . . . . . 7

2.2 Equações de Navier-Stokes . . . . . . . . . . . . . . . . . . . . 7

3 Interpolação upwind e otimização de gradientes via mínimos quadrados pon$\begin{array}{ll}\text { derados } & \mathbf{9}\end{array}$

3.1 Motivação . . . . . . . . . . . . . . . . . . . . . 9

3.2 Critérios de limitação e exemplos de esquemas upwind . . . . . . . . . . . . . . 14

4 Derivação de novos esquemas upwind $\quad 23$

4.1 Derivação de esquemas upwind via forma usual: Esquema 1 . . . . . . . . . . 23

4.2 Derivação de esquemas upwind via mínimos quadrados ponderados: os Esquemas

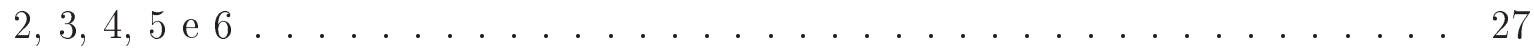

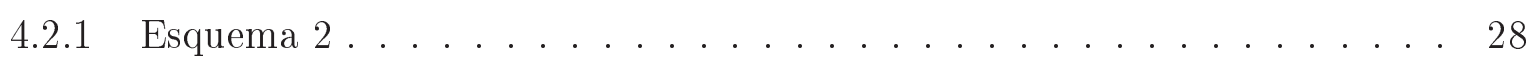

4.2 .2 Esquema $3 \ldots \ldots \ldots \ldots \ldots$

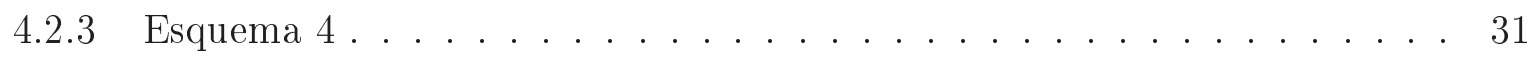

4.2 .4 Esquema 5 . . . . . . . . . . . . . . . . . . 33

4.2 .5 Esquema $6 \ldots \ldots \ldots \ldots \ldots \ldots$ 
5 Modelagem computacional $\quad 41$

5.1 Equações hiperbólicas . . . . . . . . . . . . . . . . . . . . . . . . 41

5.2 Equações de Navier-Stokes . . . . . . . . . . . . . . . . . . . . . 42

6 Resultados numéricos para leis de conservação hiperbólicas 49

6.1 Resultados para advecção de escalares . . . . . . . . . . . . . . . . . . . . 49

6.2 Resultados para equação de Burgers . . . . . . . . . . . . . . . . . . 56 56

6.3 Resultados para equação de águas rasas 1D . . . . . . . . . . . . . . . 65

6.4 Resultados para as equações de Euler 1D . . . . . . . . . . . . . . . . . . 72

6.5 Resultados para equações de águas rasas 2D . . . . . . . . . . . . . . . . . 84

6.6 Resultados para as equações de Euler 2D . . . . . . . . . . . . . . . . 86

7 Aplicação do Esquema 1 às equações de Navier-Stokes $\quad 101$

7.1 Simulação de problemas 2D e 2.5D . . . . . . . . . . . . . . . . . . 101

7.2 Simulação de problemas 3D . . . . . . . . . . . . . . . . 112

8 Conclusões e Trabalhos Futuros $\quad 123$

A Equações hiperbólicas na forma quase-linear $\quad 125$

A.1 Equação de Advecção . . . . . . . . . . . . . . . . . . . . . . . . . 125

A.2 Equação de Burgers . . . . . . . . . . . . . . . . . . . . . 126

A.3 Equações de águas rasas . . . . . . . . . . . . . . . . . . . . 126

A.4 Equações de Euler . . . . . . . . . . . . . . . . . . . . . . . 127

$\begin{array}{ll}\text { B Discretização dos termos convectivos } & 131\end{array}$

$\begin{array}{ll}\text { Referências Bibliográficas } & 134\end{array}$ 


\section{Lista de Figuras}

3.1 Definição dos pontos de interpolação $D, U$ e $R$ com respeito à interface $f=i+1 / 2$ e o sinal da velocidade de convecção $u_{(i+1 / 2, j)} \ldots \ldots \ldots$. . . . . . . . . . 11

3.2 Comparações das soluções numéricas em várias malhas (com o esquema FOU) e solução analítica para advecção de um escalar. (a) malha 200; (b) malha 400; (c) malha 800; e (d) malha 6400 .

3.3 Comparação das soluções numérica (com o esquema QUICK) e analítica numa malha computacional de 2000 pontos. . . . . . . . . . . . . . . . . . . . 13

3.4 Características NV dos esquemas convectivos FOU e QUICK; e diagrama de variáveis normalizadas, mostrando a região hachurada CBC. . . . . . . . . . . . 13

3.5 Plano $\psi(r) \perp r$ mostrando a região de extremos $\left(r_{f} \leq 0\right)$, a região de monotonicidade (vizinhança de $r_{f}=1$ ) e as regiões de alta curvatura $\left(r_{f}>>1\right.$ e $r_{f}<<1$ ). 16

3.6 Região TVD de Sweby. . . . . . . . . . . . . . . . . . . . . . . 17

3.7 Região TVD em variáveis normalizadas. . . . . . . . . . . . . . . . . . 19

4.1 Esquema 1 em variáveis normalizadas para vários valores do parâmetro $\beta$. . . 27

4.2 Limitador de fluxo correspondente do Esquema $1\left(r_{f} \geq 0\right)$ para vários valores do

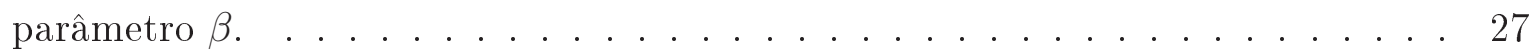

4.3 Esquema 2 em variáveis normalizadas para vários valores do parâmetro $\epsilon$. . . . 29

4.4 Limitador de fluxo correspondente do Esquema $2\left(\forall r_{f}\right)$ para vários valores do

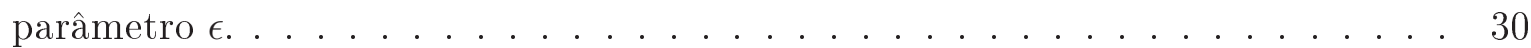

4.5 Esquema 3 em variáveis normalizadas para vários valores do parâmetro $\epsilon$. . . . 31

4.6 Limitador de fluxo correspondente do Esquema $3\left(\forall r_{f}\right)$ para vários valores do

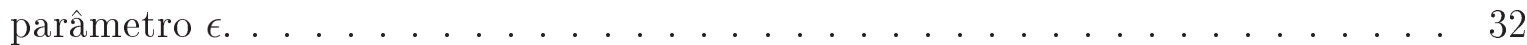

4.7 Esquema 4 em variáveis normalizadas para vários valores do parâmetro $\epsilon$. . . . 34

4.8 Limitador de fluxo correspondente do Esquema $4\left(\forall r_{f}\right)$ para vários valores do

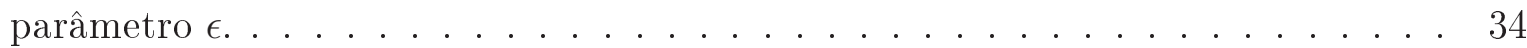

4.9 Esquema 5 em variáveis normalizadas para vários valores do parâmetro $\epsilon$. . . . 36 
4.10 Limitador de fluxo correspondente do Esquema $5\left(\forall r_{f}\right)$ para vários valores do

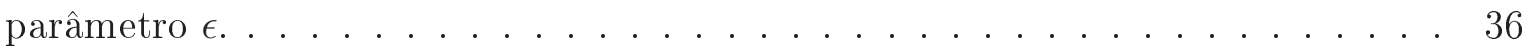

4.11 Esquema 6 em variáveis normalizadas para vários valores do parâmetro $\epsilon$. . . . 39

4.12 Limitador de fluxo correspondente do Esquema $6\left(\forall r_{f}\right)$ para vários valores do parâmetro $\epsilon \ldots \ldots \ldots \ldots \ldots \ldots \ldots$

5.1 Exemplo de uma célula computacional 3D, mostrando onde as variáveis são avaliadas.

6.1 Solução exata e resultados obtidos com os esquemas FUS-RF para advecção de um escalar. (a) Esquema 1 utilizando vários valores do parâmetro $\beta$; (b)-(f) Esquemas $2-6$ utilizando vários valores do parâmetro $\epsilon$. . . . . . . . . . . 50

6.2 Solução exata e resultados numéricos (Esquemas 1-6 com os melhores parâmetros e referências van Albada e EPUS) para advecção de um escalar - Teste 1, mostrando ampliação da região de alto gradiente. . . . . . . . . . . . . . . 5

6.3 Constante do erro para os esquemas van Albada, EPUS e Esquemas 1-6 com os melhores parâmetros, aplicados ao Teste 1.

6.4 Solução exata e resultados numéricos (Esquemas 1-6 com os melhores parâmetros e referências van Albada e EPUS) para advecção de um escalar - Teste 2, mostrando regiões de ampliações (Zoom 1-4). . . . . . . . . . . . . . . . . 55

6.5 Continuação da Figura 6.4. . . . . . . . . . . . . . . . . . . 56

6.6 Variação total para o Teste 2 com respeito ao tempo. . . . . . . . . . . 57

6.7 Evolução no tempo da solução numérica para equação de Burgers usando o esquema MC . . . . . . . . . . . . . . . . . . . . 57

6.8 Solução exata e resultados obtidos com os esquemas FUS-RF para equação de Burgers. (a) Esquema 1 utilizando vários valores do parâmetro $\beta$; (b)-(f) Esquemas $2-6$ utilizando vários valores do parâmetro $\epsilon$.

6.9 Comparação (antes e depois do choque) entre a solução exata e as soluções numéricos (Esquemas 1-6 com os melhores parâmetros e referências van Albada e EPUS) para equação de Burgers - Teste 3, mostrando regiões de ampliação. . 60

6.10 Continuação da Figura 6.9. . . . . . . . . . . . . . . . . . 60

6.11 Constante de erro $C$ na norma $L_{1}$ para o Teste 3 antes e depois do choque. . . 64

6.12 Comparação das soluções numéricas e aolução exata para o Teste 4 usando os esquema van Albada, EPUS e Esquemas 1-6 com os melhores parâmetros, mostrando regiões de ampliação. . . . . . . . . . . . . . . . . . . . . . . 64

6.13 Continuação da Figura 6.12. . . . . . . . . . . . . . . . . . . . . . 64

6.14 Variação total para o Teste 4 com respeito ao tempo. . . . . . . . . . . . . 65 
6.15 Comparação das soluções numéricas e aolução de referência para a altura $h$ do fluido no Teste 5 usando os esquema van Albada, EPUS e Esquemas 1-6 com os melhores parâmetros, mostrando regiões de ampliação. . . . . . . . . . . . . 66

6.16 Continuação da Figura 6.15. . . . . . . . . . . . . . . . . . 66

6.17 Comparação das soluções numéricas e solução de referência para a vazão $h u$ do fluido no Teste 5 usando os esquema van Albada, EPUS e Esquemas 1-6 com os melhores parâmetros, mostrando regiões de ampliação. . . . . . . . . . . . . . 67

6.18 Continuação da Figura 6.17. . . . . . . . . . . . . . . . . . . 67

6.19 Variação total em várias malhas para o Teste 5 com respeito ao tempo. . . . . . 68

6.20 Comparação das soluções numéricas e solução de referência para a vazão $h u$ do fluido no Teste 6 usando os esquema van Albada, EPUS e Esquemas 1-6 com os melhores parâmetros, mostrando regiões de ampliação. . . . . . . . . . . . . . 69

6.21 Continuação da Figura 6.20 . . . . . . . . . . . . . . . . . . . . . . . . . 70

6.22 Comparação das soluções numéricas e solução de referência para a altura $h$ do fluido no Teste 6 usando os esquema van Albada, EPUS e Esquemas 1-6 com os melhores parâmetros, mostrando regiões de ampliação. . . . . . . . . . . . . . 70

6.23 Continuação da Figura 6.22. . . . . . . . . . . . . . . . . . . . . . 71

6.24 Comparação das soluções numéricas e a solução de referência para a pressão $p$ no Teste 7, usando os esquema van Albada, EPUS e Esquemas 1-6 com os melhores parâmetros, mostrando regiões de ampliação. . . . . . . . . . . . . . . . . 72

6.25 Continuação da Figura 6.24. . . . . . . . . . . . . . . . . . . 73

6.26 Comparação das soluções numéricas e a solução de referência para a densidade $\rho$ no Teste 7, usando os esquema van Albada, EPUS e Esquemas 1-6 com os melhores parâmetros, mostrando regiões de ampliação. . . . . . . . . . . . . 73

6.27 Continuação da Figura 6.26. . . . . . . . . . . . . . . . . . . . . 73

6.28 Comparação das soluções numéricas e a solução de referência para a velocidade $u$ no Teste 7, usando os esquema van Albada, EPUS e Esquemas 1-6 com os melhores parâmetros, mostrando regiões de ampliação. . . . . . . . . . . . . . . 74

6.29 Continuação da Figura 6.28. . . . . . . . . . . . . . . . . . . . . . . . . . . . 74

6.30 Comparação das soluções numéricas e a solução de referência para a energia $E$ no Teste 8, usando os esquema van Albada, EPUS e Esquemas 1-6 com os melhores parâmetros, mostrando regiões de ampliação. . . . . . . . . . . . . . . . . 77

6.31 Continuação da Figura 6.30 . . . . . . . . . . . . . . . . . . . 77

6.32 Comparação das soluções numéricas e a solução de referência para a velocidade $u$ no Teste 8, usando os esquema van Albada, EPUS e Esquemas 1-6 com os melhores parâmetros, mostrando regiões de ampliação. . . . . . . . . . . . 78

6.33 Continuação da Figura 6.32. . . . . . . . . . . . . . . . . . . . . . . 78 
6.34 Comparação das soluções numéricas e a solução de referência para a densidade $\rho$ no Teste 8, usando os esquema van Albada, EPUS e Esquemas 1-6 com os melhores parâmetros, mostrando regiões de ampliação. . . . . . . . . . . . . . . 79

6.35 Continuação da Figura 6.34. . . . . . . . . . . . . . . . . . . . . . . . 79

6.36 Variação total da variável conservada $E$ para o Teste 8 em várias malhas com respeito ao tempo. . . . . . . . . . . . . . . . . . 8

6.37 Comparação das soluções numéricas e a solução de referência para a pressão $p$ no Teste 9, usando os esquema van Albada, EPUS e Esquemas 1-6 com os melhores parâmetros, mostrando regiões de ampliação. . . . . . . . . . . . . . . . . . 81

6.38 Continuação da Figura 6.37. . . . . . . . . . . . . . . . . . . . . . . . 81

6.39 Comparação das soluções numéricas e a solução de referência para a densidade $\rho$ no Teste 9, usando os esquema van Albada, EPUS e Esquemas 1-6 com os melhores parâmetros, mostrando regiões de ampliação. . . . . . . . . . . . . . . 82

6.40 Continuação da Figura 6.39. . . . . . . . . . . . . . . . . . . . . . 82

6.41 Comparação das soluções numéricas e a solução de referência para a velocidade $u$ no Teste 9, usando os esquema van Albada, EPUS e Esquemas 1-6 com os melhores parâmetros, mostrando regiões de ampliação. . . . . . . . . . . . . . 83

6.42 Continuação da Figura 6.41. . . . . . . . . . . . . . . . . . . . . 83

6.43 Comportamento da altura $h$ da porção de fluido nos instantes $t=0$ e $t=0.25$. Figura extraída de LeVeque $[21] \ldots \ldots$. . . . . . . . . . . . . . . 84

6.44 Solução de referência e resultados obtidos com os esquemas FUS-RF sob a reta $y=0$. (a) Esquema 1 utilizando vários valores do parâmetro $\beta$; (b)-(f) Esquemas $2-6$ utilizando vários valores do parâmetro $\epsilon$. . . . . . . . . . . . . . .

6.45 Solução de referência e resultados numéricos (Esquemas 1-6 com os melhores parâmetros, van Albada e EPUS) para o Teste 10, mostrando região de ampliação. 86

6.46 Perfil para a altura $h$ do fluido no Teste 10. (a) van Albada; (b) EPUS; (c)-(h) Esquemas 1-6 com os melhores parâmetros. . . . . . . . . . . . . . . 87

6.47 Continuação da Figura 6.46. . . . . . . . . . . . . . . . . . . . 88

6.48 Solução exata e resultados obtidos com os esquemas FUS-RF para as equações de Euler - Teste 11. (a) Esquema 1 utilizando vários valores do parâmetro $\beta$; (b)-(f) Esquemas $2-6$ utilizando vários valores do parâmetro $\epsilon$. . . . . . . . . 90

6.49 Comparação entre a solução exata e as soluções numéricos (Esquemas 1-6 com os melhores parâmetros e referências van Albada e EPUS) para as equações de Euler - Teste 11, mostrando região de ampliação. . . . . . . . . . . . . . . . . . 91

6.50 Solução de referência e resultados obtidos com os esquemas FUS-RF sob a reta $x=y$ com regiões de ampliação . (a) Esquema 1 utilizando vários valores do parâmetro $\beta$; (b)-(f) Esquemas $2-6$ utilizando vários valores do parâmetro $\epsilon . \quad$. 94 
6.51 Solução de referência e resultados numéricos (Esquemas 1-6 com os melhores parâmetros, van Albada e EPUS) para o Teste 12, mostrando região de ampliação (zoom 1 - zoom 4). . . . . . . . . . . . . . . . . . . . 95

6.52 Continuação da Figura 6.51. . . . . . . . . . . . . . . . . . . 95

6.53 Perfil para a densidadde $\rho$ do fluido no Teste 12. (a) van Albada; (b) EPUS; (c)-(h) Esquemas 1-6 com os melhores parâmetros. . . . . . . . . . . . . . 96

6.54 Continuação da Figura 6.53. . . . . . . . . . . . . . . . . . . . 97

6.55 Perfil para a densidadde $\rho$ do fluido no Teste 13. (a) WENO5 (b) van Albada; (c) EPUS; (d)-(i) Esquemas 1-6 com os melhores parâmetros na malha 240 $\times 960$ (grossa) . . . . . . . . . . . . . . . . . . .

6.56 Perfil para a densidade $\rho$ do fluido no Teste 13. (a) WENO5 (b) van Albada; (c) EPUS; (d)-(i) Esquemas 1-6 com os melhores parâmetros na malha $480 \times 1920$ (fina).

7.1 Esquematização de um jato livre incidindo perpendicularmente sobre uma superfície rígida impermeável . . . . . . . . . . . . . . . . . 102

7.2 Contornos da velocidade em x, em y e da pressão para o problema do jato livre em diferentes tempos. . . . . . . . . . . . . . . . . . . 103

7.3 Comparação entre a solução analítica de Watson [51] e a solução numérica para diferentes malhas. . . . . . . . . . . . . . . . . . . . 104

7.4 Esquematização do problema de colapso de uma coluna de fluido 2D. . . . . . . 104

7.5 Comparação entre as soluções experimentais, numéricas e teóricas para o fenômeno do colapso de uma coluna de fluido. . . . . . . . . . . . . . 105

7.6 Contornos da velocidade em x, em y e da pressão para o problema do colapso de uma coluna de fluido em 2D para diferentes tempos. . . . . . . . . . . . 106

7.7 Esquematização para o experimento de Taylor. . . . . . . . . . . . . . . 107

7.8 Ilustração do experimento de Taylor em diferentes tempos. (a) e (c) solução experimental; (b) e (d) solução numérica. . . . . . . . . . . . . . . . . . 108

7.9 Ilustração da solução para o experimento de Taylor em diferentes tempos. . . . . 109

7.10 Ilustração do experimento de Taylor em $T=7 \mathrm{~s}$ e corte transversal evidenciando a formação de estruturas complexas. . . . . . . . . . . . . . . . 109

7.11 Esquematização para o fenômeno do ressalto hidráulico circular. . . . . . . . . . 110

7.12 Comparação entre a solução analítica e a solução numérica para o ressalto hidráulico circular, considerando diferentes malhas. . . . . . . . . . . . . . 111

7.13 Ilustração do ressalto hidráulico circular. (a) solução experimental de Rai [34] (b) solução numérica. . . . . . . . . . . . . . . . . . . . . . . . . . . . 111

7.14 Esquematização para o colapso de fluido 3D . . . . . . . . . . . . . . . . 112

7.15 Comparação entre as soluções experimentais, numéricas e teóricas para o fenômeno do colapso de uma coluna de fluido em 3D. 
7.16 Campos de pressão e de velocidade em x e z para o problema do colapso de uma coluna de fluido em 3D para diferentes tempos. . . . . . . . . . . . . . . 114

7.17 Esquematização para o problema do jato planar 3D . . . . . . . . . . . . . 115

7.18 Ilustração da solução para o problema do jato planar em 3D para diferentes tempos. 116

7.19 Esquematização para o problema do jato circular em $3 D$. . . . . . . . . . . . 117

7.20 Ilustração da solução para o problema do jato circular em 3D para diferentes tempos-Caso 1. . . . . . . . . . . . . . . . . . . . . 118

7.21 Ilustração do jato circular oscilante em 3D para diferentes tempos - Caso 2. (a) solução experimental; (b) solução numérica. . . . . . . . . . . . . . . . . . . . . 119

7.22 Ilustração do fenômeno do ressalto hidráulico circular- Caso 1. (a) solução experimental de Rai [34] ; (b) solução numérica. . . . . . . . . . . . . . . . . . 120

7.23 Ilustração da solução para o fenômeno do ressalto hidráulico em diferentes tempos para $R_{e}=250-$ Caso 1. . . . . . . . . . . . . . . . . . . 121

7.24 Ilustração do fenômeno do ressalto hidráulico circular- Caso 2. (a) solução experimental de Ellergard [9] ; (b) solução numérica. . . . . . . . . . . . . . . . 122

7.25 Ilustração da solução para o fenômeno do ressalto hidráulico em diferentes tempos para $R_{e}=1000-$ Caso 2. . . . . . . . . . . . . . . 122 


\section{Lista de Tabelas}

6.1 Comparação dos erros nas normas $L_{1}, L_{2}$ e $L_{\infty}$ para o Teste 1 em várias malhas, mostrando os ordens observadas e as constantes do erro. Os resultados são para os esquemas van Albada, EPUS e os Esquemas 1-6 com os melhores parâmetros. 53

6.2 Continuação da Tabela 6.1. . . . . . . . . . . . . . . . . . . . 54

6.3 Comparação dos erros na norma $L_{2}$ para o Teste 3 em várias malhas, mostrando (antes e depois do choque) as ordens observadas e as constantes do erro. Os resultados são para os esquemas van Albada, EPUS e os Esquemas 1-6 com vários parâmetros. . . . . . . . . . . . . . . . . . 6 61

6.4 Continuação da Tabela 6.3. . . . . . . . . . . . . . . . . . . . 62

6.5 Continuação da Tabela 6.4. . . . . . . . . . . . . . . . . . . . 63

6.6 Comparação dos erros e ordens observadas nas normas $L_{1}, L_{2}$ e $L_{\infty}$ para o Teste 6, usando os esquemas van Albada, EPUS e os Esquemas 1-6 com os melhores parâmetros. . . . . . . . . . . . . . . . . 71

6.7 Comparação dos erros e ordens observadas nas normas $L_{1}, L_{2}$ e $L_{\infty}$ para o Teste 7, usando os esquemas van Albada, EPUS e os Esquemas 1-6 com vários parâmetros. . . . . . . . . . . . . . . . . . 75

6.8 Continuação da Tabela 6.7. . . . . . . . . . . . . . . . . . . . 76

6.9 Comparação dos erros e ordens observadas nas normas $L_{1}, L_{2}$ e $L_{\infty}$ para o Teste 11, usando os esquemas van Albada, EPUS e os Esquemas 1-6 com vários parâmetros. . . . . . . . . . . . . . . . . . . . . 9 91

6.10 Continuação da Tabela 6.9. . . . . . . . . . . . . . . . . . . . . 92

6.11 Continuação da Tabela 6.10 . . . . . . . . . . . . . . . . . . . . . 93 
CAPÍTULO

\section{1}

\section{Introdução}

Muitos problemas em fluidodinâmica computacional (ou dinâmica dos fluidos computacional) podem ser modelados por equações diferenciais à derivadas parciais (EDPs), comumente conhecidas como equações de conservação. Exemplos representativos são as equações de Euler (para a dinâmica de gases) e Navier-Stokes (para fluidos viscosos) em condições adversas de convecção. Soluções clássicas para essas EDPs contêm descontinuidades (ou choques), camadas limite e não unicidade, características essas, principalmente nos casos não lineares predominantemente convectivos, que fazem da simulação computacional um trabalho bastante complexo.

Muitas técnicas numéricas consagradas na literatura falham, mesmo em EDPs lineares com caráter convectivo moderado. A extensão aos casos multidimensionais não lineares com caráter convectivo acentuado torna-se um trabalho muito mais complexo, uma vez que podem aparecer instabilidades (físicas/não físicas) acarretando não convergência ou violação de entropia. Devido a essas dificuldades, uma parte substancial da comunidade científica, principalmente em fluidodinâmica computacional, tem se dedicado ao desenvolvimento e teste de métodos numéricos capazes de resolver EDPs de caráter predominantemente convectivo. Tais métodos devem, em particular, fornecer soluções fisicamente corretas, com boa resolução nas vizinhanças de descontinuidades e sem introduzir oscilações numéricas ou suavizações.

A fim de derivar métodos computacionais que atendam às condições de estabilidade e convergência, sem introduzir oscilações espúrias e com pouca suavização, Harten [14] introduziu o conceito de esquemas de alta resolução TVD (Total Variation Diminishing), os quais foram posteriormente estendidos, admitindo-se esquemas completamente discretos ou semi-discretos, por Swebby, Chakravarthy-Osher, Shu-Osher, Jameson, Schmidt, Turkel, entre outros (ver [21]). Jin e Xin [15], por exemplo, em 1995 propuseram esquemas de relaxação reduzindo as equações de conservação à sistemas de conservações lineares hiperbólicos com termo fonte. Em combinação à restrição TVD, Leonard [19] propôs uma normalização de variáveis e forneceu 
condições para se obter estabilidade não linear. As condições de Leonard agregadas à definição de limitadores de fluxo (ver [41]) e ao critério de limitação CBC (Convection Boundedness Criterion) de Gaskell e Lau [12] possibilitaram a derivação de esquemas upwind que atendam às necessidades de estabilidade, de limitação e de convergência.

Uma característica importante das equações de conservação com caráter predominantemente convectivo (principalmente as leis de conservação hiperbólicas) é que as informções físicas se propagam por meio das características. Métodos numéricos que herdam esta propriedade (comumente chamados esquemas convectivos ou upwind) são, portanto, os mais indicados nesses casos. Vários esquemas convectivos upwind foram produzidos nas últimas décadas, dentre eles destacam-se o esquema HLPA (Hybrid Linear Parabolic Approximation) de Zhu [55], o SMART (Sharp and Monotonic Algorithm for Realistic Transport) de Gaskell e Lau [12], o WACEB (Weighted-Average Coefficient Ensuring Boundedness) de Song et al. [40]. Mais recentemente, Alves et al. [24] propuseram o esquema CUBISTA (Convergent and Universally Bounded Interpolation Scheme for the Treatment of advection), Ferreira et al. [10] desenvolveram o esquema ADBQUICKEST (ADaptative Bounded QUICKEST), uma versão limitada do esquema QUICKEST (QUICK with Estimated Streaming Terms) de Leonard [17]; e Ferreira et al. [33] intoduziram o esquema polinomial TOPUS (Third-Order Polynomial Upwind Scheme) para simular problemas compressíveis e incompressíveis.

\subsection{Motivação e objetivos}

Nos últimos anos os pesquisadores do LMACC (Laboratório de Matemática Aplicada e Computação Científica) do ICMC/USP têm se dedicado ao desenvolvimento de métodos numéricos em dinâmica dos fluidos computacional para simular escoamentos incompressíveis a uma ampla faixa do número de Reynolds. Em particular, várias técnicas inovadoras foram desenvolvidas e inseridas no contexto dos projetos temáticos da FAPESP, como Solução numérica das equações de Navier-Stokes e Mecânica dos fluidos não estacionária: aplicação em aeronáutica e em reologia, dentre elas destacam-se:

- Desenvolvimento, análise e implementação de esquemas upwind de alta resolução para leis de conservação hiperbólicas gerais;

- Estudo, análise e implementação de esquemas upwind de alta resolução para escoamentos 2D e 3D de fluidos newtonianos no regime laminar e com superfícies livres móveis;

- Implementação da modelagens da turbulência, tais como $\kappa-\varepsilon$ e RNG;

- Implementação de modelos reológicos, tais como Maxell, PTT, Oldroyd-B, dentre outros.

Estes avanços foram parcialmente incorporados ao código Freeflow [4], permitindo a simulação de escoamentos com superfícies livres móveis (ver [10], [11], [37]) a vários números 
de Reynolds e de escoamentos reológicos tridimensionais de grande complexidade e interesse tecnológico na indústria de polímeros (ver, por exemplo, [29], [25]).

Apesar dos resultados satisfatórios gerados pelo sistema Freeflow, algumas dificuldades ainda impedem a simulação de alguns fenômenos em dinâmica dos fluidos para problemas de interesse acadêmico/tecnológico. Desta forma, considera-se imprescindível, para aumentar a aplicabilidade das ferramentas desenvolvidas, um esforço de integração e uma metodologia numérica única para simular escoamentos laminares a altos Reynolds e turbulentos de fluidos newtonianos com superfícies livres móveis.

Dentro deste cenário, propõe-se neste trabalho uma família de funções racionais denominada FUS-RF (Family of Upwind Schemes via Rational Functions), para a simulação computacional de equações de conservação com caráter predominante convectivo. A família FUS-RF é composta de seis esquemas upwind dependentes de parâmetros, que foi desenvolvida com base nos critérios de estabilidade TVD/CBC e na técnica de minimização mínimos quadrados ponderados.

A família FUS-RF é utilizada inicialmente para simular leis de conservação hiperbólicas, à saber, equação de advecção, equação não linear de Burgers, águas rasas e equações de Euler nos casos 1D e 2D. Para a simulação desses problemas, utiliza-se o pacote computacional CLAWPACK (Conservation LAWs PACKage) desenvolvido por LeVeque [21]. O código, implementado em linguagem Fortran, resolve numericamente uma variedade de sistemas hiperbólicos, desde advecção de escalares até sistemas hiperbólicos tridimensionais. Este código usa o método de volumes finitos, permitindo resolver problemas de Riemann com condições iniciais e de contorno bem definidas. No software serão incluídos os limitadores de fluxo FUS-RF desenvolvidos neste trabalho. Os resultados numéricos serão comparados com os resulatdos obtidos com os limitadores de fluxo do bem estabelecido esquema van Albada [46] e do atual EPUS [7]. Os esquemas racionais serão classificados e, então, o mais robusto será utilizado na simulação computacional de escoamentos laminares de fluidos newtonianos incompressíveis com superfícies livres móveis. O ambiente Freeflow de Castelo et al. [4] é o código base para as simulações dos problemas de escoamento de fluidos viscosos. Este código utiliza o método de diferenças finitas sobre malhas diferenciadas. A metodologia numérica de solução a ser utilizada é uma variante do método de projeção de Chorin [5] proposto originalmente por Harlow e Welch [13] (Método MAC) e bem discutido por Peyret e Taylor [31].

\subsection{Organização do texto}

Os capítulos desta dissertação estão dispostos da seguinte maneira:

- O capítulo 2 apresenta a formulação matemática de equações de conservação lineares e não lineares consideradas neste trabalho. São apresentadas também as condições inicias e de contorno adotadas; 
- No capítulo 3 é feita uma contextualização da proposta e são apresentadas condições para determinação de esquemas upwind nos contextos TVD e CBC. Apresenta-se o conceito de minimização via técnica de mínimos quadrados ponderados para a derivação de limitadores de fluxo otimizados.

- No capítulo 4 são propostos novos esquemas upwind da família FUS-RF. Uma análise das propriedades descritas no capítulo 3 é também apresentada;

- No capítulo 5 apresenta-se a técnica computacional para aproximação dos termos convectivos das equações consideradas neste trabalho. Apresentam-se também a estrutura de resolução do software CLAWPACK equipado com novos limitadores de fluxo, e a estrutura do ambiente de simulação Freeflow adaptado com os novos esquemas da família FUS-RF.

- O capítulo 6 contempla os resultados computacionais para leis de conservação hiperbólicas 1D e 2D. Apresenta-se também uma análise comparativa dos novos esquemas e os esquemas van Albada e EPUS.

- No sétimo capítulo um esquema particular dessa nova família de esquemas upwind é utilizado para resolver escoamentos incompressíveis complexos com superfícies livres móveis.

- As conclusões deste trabalho, bem como os planos para o futuro, são apresentadas no capítulo 8. 
CAPÍTULO

\section{Formulação matemática}

Neste capítulo apresentam-se as equações matemáticas consideradas no presente trabalho.

\subsection{Equações de conservação hiperbólicas}

Equações diferenciais parciais (EDPs) do tipo hiperbólico modelam uma grande variedade de fenômenos físicos que envolvem transporte de substâncias ou movimento de ondas. Estas são geralmente não lineares e dependentes do tempo. O caso $\mathrm{n}$ dimensional na forma quase-linear é representado por

$$
\frac{\partial \phi}{\partial t}+A_{1} \frac{\partial \phi}{\partial x_{1}}+A_{2} \frac{\partial \phi}{\partial x_{2}}+\cdots+A_{n} \frac{\partial \phi}{\partial x_{n}}=\zeta
$$

onde $\phi\left(x_{1}, x_{2}, \cdots, x_{n}, t\right): \mathbb{R}^{n} \times \mathbb{R} \rightarrow \mathbb{R}^{m}$ é o vetor das incógnitas do problema (por exemplo, pressão, velocidade, ...) a ser determinado, e $A_{i_{m \times m}}$ uma matriz real, diagonalizável e com autovalores reais (ver LeVeque, [21]); $\zeta$ são termos fonte. Em variáveis conservadas, o sistema (2.1) é escrito como

$$
\frac{\partial \phi}{\partial t}+\frac{\partial F_{i}(\phi)}{\partial x_{i}}=\zeta, \quad i=1, \ldots, n,
$$

onde $F_{i}(\phi)=F_{i}\left(\phi\left(x_{1}, x_{2}, \cdots, x_{n}, t\right)\right): \mathbb{R}^{m} \rightarrow \mathbb{R}^{m}$ são as funções fluxo.

Alguns casos particulares das equações (2.2) são apresentados na sequência.

\subsubsection{Equação linear de advecção}

A equação linear de advecção 1D é o caso mais simples de leis de conservação hiperbólicas e modela o transporte de um escalar com uma velocidade prescrita. Nesta equação a variável conservada $\phi$ é transportada com velocidade advectiva $u$, e o termo fonte é assumido nulo, isto 
é

$$
\frac{\partial \phi}{\partial t}+\frac{\partial F_{1}(\phi)}{\partial x}=0, \quad x \in\left[x_{L}, x_{R}\right], \quad t \in[0, T],
$$

onde $F_{1}(\phi)=u \phi$. A solução exata deste problema é um deslocamento da solução inicial $\phi_{0}$ dada por

$$
\phi(x, t)=\phi_{0}(x-u t)
$$

Condições de contorno periódicas são comumente utilizadas neste caso.

\subsubsection{Equação não linear de Burgers}

A equação não linear de Burgers possui características comuns às equações que modelam fluidos em movimento (equações Navier-Stokes), porém, por ser mais simples que estas últimas, é utilizada frequentemente em estudos numéricos como teste preliminar. No caso 1D, a equação (2.2) torna-se

$$
\frac{\partial \phi}{\partial t}+\frac{\partial F_{1}(\phi)}{\partial x}=\zeta, \quad x \in\left[x_{L}, x_{R}\right], \quad t \in[0, T]
$$

onde $F_{1}(\phi)=\frac{\phi^{2}}{2}$. Assume-se, neste caso, $\zeta=0$ (caso não viscoso) ou $\zeta=\nu \frac{\partial^{2} \phi}{\partial x^{2}}$ (caso viscoso), $\nu$ sendo o coeficiente de viscosidade (constante).

\subsubsection{Equações de águas rasas}

As equações de águas rasas modelam fenômenos meteorológicos e oceanográficos envolvendo ondas de superfície, em que a altura da onda é pequena comparada com o seu comprimento. No caso 1D, o sistema de equações (2.2) torna-se

$$
\frac{\partial \phi}{\partial t}+\frac{\partial F_{1}(\phi)}{\partial x}=0, \quad x \in\left[x_{L}, x_{R}\right], \quad t \in[0, T]
$$

onde $\phi=[h, h u]^{T}$ e $F_{1}=\left[h u, h u^{2}+\frac{1}{2} g h^{2}\right]^{T}$; sendo $h=h(x, t)$ a profundidade do fluido num canal, $u$ a velocidade horizontal do fluido, $h u$ a vazão e $g=9.81 \mathrm{~m} / \mathrm{s}^{2}$ a constante gravitacional. Para condições de contorno, em geral, usa-se extrapolação de ordem zero (ver, por exemplo, [21]). No caso 2D, o sistema de equações (2.2) é

$$
\frac{\partial \phi}{\partial t}+\frac{\partial F_{1}(\phi)}{\partial x}+\frac{\partial F_{2}(\phi)}{\partial y}=0, \quad x \in\left[x_{L}, x_{R}\right], \quad y \in\left[y_{B}, y_{T}\right], \quad t \in[0, T]
$$

onde $\phi=[h, h u, h v]^{T}, F_{1}=\left[h u, h u^{2}+\frac{1}{2} g h^{2}, h u v\right]^{T}$ e $F_{2}=\left[h v, h u v, h v^{2}+\frac{1}{2} g h^{2}\right]^{T}$; sendo $v$ a velocidade vertical. Como no caso 1D, usa-se extrapolação de ordem zero no contorno. 


\subsubsection{Equações de Euler}

As equações de Euler modelam a dinâmica de gases. No caso 1D, o sistema de equações (2.2) torna-se

$$
\frac{\partial \phi}{\partial t}+\frac{\partial F_{1}(\phi)}{\partial x}=\zeta, \quad x \in\left[x_{L}, x_{R}\right], \quad t \in[0, T],
$$

onde $\phi=[\rho, \rho u, E]^{T}$ e $F_{1}=\left[\rho u, \rho u^{2}+p, u(E+p)\right]^{T}$; sendo $\rho$ a densidade, $p$ a pressão, $\rho u$ a quantidade de movimento e $E$ a energia total. A equação de gás ideal $p=(\gamma-1)\left(E-\frac{1}{2} \rho u^{2}\right)$ é usada para fechar o problema, em que $\gamma$ é a razão do calor específico. As condições de contorno comumente usadas são extrapolação de ordem zero. No caso 2D, o sistema de equações (2.2) torna-se

$$
\frac{\partial \phi}{\partial t}+\frac{\partial F_{1}(\phi)}{\partial x}+\frac{\partial F_{2}(\phi)}{\partial y}=\zeta, \quad x_{1} \in\left[x_{L}, x_{R}\right], \quad y \in\left[y_{B}, y_{T}\right], \quad t \in[0, T]
$$

em que $\phi=[\rho, \rho u, \rho v, E]^{T}, F_{1}=\left[\rho u, \rho u^{2}+p, \rho u v, u(E+p)\right]^{T}$ e $F_{2}=\left[\rho v, \rho u v, \rho v^{2}+p, v(E+p)\right]^{T}$; sendo a pressão $p$ definida em função de $E$ pela relação de fechamento para gás ideal $p=$ $(\gamma-1)\left(E-\frac{1}{2} \rho\left(u^{2}+v^{2}\right)\right)$. As condições de contorno comumente usadas são extrapolação de ordem zero.

\subsection{Equações de Navier-Stokes}

As equações de Navier-Stokes, em coordenadas cartesianas, que modelam escoamentos incompressíveis de fluidos newtonianos são, na forma adimensional, dadas pela equação de conservação da massa

$$
\nabla \cdot \mathbf{u}=\mathbf{0}
$$

e a conservação da quantidade de movimento

$$
\frac{\partial \mathbf{u}}{\partial t}+\nabla \cdot \mathbf{u} \mathbf{u}=-\nabla \cdot p+\frac{1}{R_{e}} \nabla^{2} \mathbf{u}+\frac{1}{F_{r}^{2}} \mathbf{g}
$$

em que $\mathbf{u}$ é campo de velocidades, $\mathbf{u}=[u, v]^{T}$ para o caso $2 \mathrm{D}$ e $\mathbf{u}=[u, v, w]^{T}$ para o caso $3 \mathrm{D}$, onde $u, v$ e $w$ são as velocidades em $x, y$ e $z$ respectivamente. Os números adimensionais de Reynolds e Froude são, respectivamente, definidos como $R_{e}=L_{0} U_{0} / \nu$ e $F_{r}=U_{0} / \sqrt{L_{0}|\mathbf{g}|}$; sendo $L_{0}$ e $U_{0}$ as escalas de comprimento e velocidade, respectivamente. Outras variáveis e constantes foram definidas previamente.

As equações de Navier-Stokes em coordenadas cilíndricas são dadas por

$$
\begin{gathered}
\frac{\partial u}{\partial t}+\frac{1}{r} \frac{\partial(r u u)}{\partial r}+\frac{\partial(u v)}{\partial z}=-\frac{\partial p}{\partial r}+\frac{1}{R_{e}} \frac{\partial}{\partial z}\left(\frac{\partial u}{\partial z}-\frac{\partial v}{\partial r}\right)+\frac{1}{F_{r}^{2}} g_{r}, \\
\frac{\partial v}{\partial t}+\frac{1}{r} \frac{\partial(r v u)}{\partial r}+\frac{\partial(v v)}{\partial z}=-\frac{\partial p}{\partial z}+\frac{1}{R_{e}} \frac{1}{r} \frac{\partial}{\partial r}\left(r\left(\frac{\partial u}{\partial z}-\frac{\partial v}{\partial r}\right)\right)+\frac{1}{F_{r}^{2}} g_{z},
\end{gathered}
$$




$$
\frac{1}{r} \frac{\partial(r u)}{\partial r}+\frac{\partial v}{\partial z}=0
$$

em que $u=u(r, z, t)$ e $v=v(r, z, t)$ são os componentes do campo de velocidades; $r$ e $z$ representam, respectivamente, a coordenada radial e o eixo de simetria.

As condições de contorno aplicadas para resolver as equações de Navier-Stokes são, em geral, como segue:

- Injetor: Nesse contorno são prescritas as velocidades normal ao contorno $\left(u_{n}\right)$ e tangencial ao contorno $\left(u_{t}\right)$ (Condição de Dirichlet)

$$
u_{n}=U_{0}, \quad u_{t}=0
$$

- Ejetor: Nesse contorno considera-se a condição homogênea de Neumann

$$
\frac{\partial u_{n}}{\partial n}=\frac{\partial u_{t}}{\partial n}=0
$$

- Contorno rígido: Neste contorno são aplicadas as condições de não-escorregamento

$$
u_{n}=u_{t}=0
$$

ou escorregamento

$$
u_{n}=0, \frac{\partial u_{t}}{\partial n}=0
$$

- Superfície livre: Na superfície livre assume-se que o fluido está em uma atmosfera passiva, isto é

$$
\begin{aligned}
& n \cdot(\sigma \cdot n)=0, \\
& m_{1} \cdot(\sigma \cdot n)=0, \\
& m_{2} \cdot(\sigma \cdot n)=0,
\end{aligned}
$$

onde $n$ é o vetor normal à superfície, $m_{1}$ e $m_{2}$ os vetores tangenciais e $\sigma$ é o tensor das tensões dado por

$$
\sigma=-p I+2 \nu\left(\nabla u+(\nabla u)^{t}\right) .
$$




\section{Interpolação upwind e otimização de gradientes via mínimos quadrados ponderados}

Neste capítulo apresentam-se relações importantes para o desenvolvimento de métodos upwind de alta resolução. Objetiva-se aproximar termos convectivos (lineares e não lineares) de leis de conservação gerais. É apresentado também uma forma de se estimar o gradiente de forma otimizada, por meio da técnica mínimos quadrados ponderados.

\subsection{Motivação}

Por simplicidade, e sem perda de generalidade, considera-se a equação (2.3) avaliada em um ponto $\left(x_{i}, t_{j}\right)=\left(i \delta_{x}, j \delta_{t}\right)=(i, j)$ da malha, onde $\delta_{x}$ é o espaçamento de malha (assumido neste trabalho como constante ao longo do domínio), e $\delta_{t}$ é o tamanho do passo temporal, assim

$$
\left(\frac{\partial \phi}{\partial t}+\frac{\partial(u \phi)}{\partial x}\right)_{(i, j)}=\left(\frac{\partial \phi}{\partial t}\right)_{(i, j)}+\left(\frac{\partial(u \phi)}{\partial x}\right)_{(i, j)}=0
$$

Uma aproximação para a derivada temporal pode ser feita utilizando-se algum método numérico para EDO, como Euler ou Runge-Kutta. Para o termo espacial, usa-se a seguinte aproximação

$$
\left(\frac{\partial(u \phi)}{\partial x}\right)_{(i, j)} \approx \frac{(u \phi)_{(i-1 / 2, j)}-(u \phi)_{(i+1 / 2, j)}}{\delta x}=\frac{1}{\delta x}\left\{u_{(i-1 / 2, j)} \phi_{(i-1 / 2, j)}-u_{(i+1 / 2, j)} \phi_{(i+1 / 2, j)}\right\} .
$$

Note que para avaliar o termo espacial no ponto $(i, j)$ é necessário conhecer a velocidade de convecção $u$, bem como a variável transportada $\phi$, nos pontos (não de malha) $(i-1 / 2, j$ ) e $(i+1 / 2, j)$. As velocidades de convecção $u$ nesses pontos (ou nas faces $g=(i-1 / 2)$ e 
$f=(i+1 / 2))$ são calculadas por meio da média simples

$$
u_{(i-1 / 2, j)}=\frac{1}{2}\left(u_{(i, j)}+u_{(i-1, j)}\right), \quad u_{(i+1 / 2, j)}=\frac{1}{2}\left(u_{(i+1, j)}+u_{(i, j)}\right) .
$$

Os valores da variável $\phi$ nos pontos $(i-1 / 2, j)$ e $(i+1 / 2, j)$ (que são chamados fluxos numéricos nas faces $g$ e $f$ ) não são conhecidos, e portanto precisam ser determinados por alguma técnica de interpolação. Uma maneira interessante e útil de se obter aproximações para esses fluxos é fazer uma interpolação levando em conta o sinal da velocidade de convecção nessas faces. Tal procedimento é conhecido como interpolação upwind. De posse do sinal da velocidade de convecção $u$ perpendicular a uma interface da célula computacional, por exemplo $f=i+1 / 2$, a interpolação upwind da variável $\phi$ é feita em função dos três pontos vizinhos de malha mais próximos, a saber: D (Downstream), ponto à jusante da interface $f$; U ( Upstream), ponto à montante da interface $f$; e $\mathrm{R}$ (Remote upstream), ponto mais à montante de $f$. Em outras palavras, a variável convectada $\phi$ na interface $f$ é uma função da forma

$$
\phi_{f}=\phi\left(\phi_{D}, \phi_{U}, \phi_{R}, \operatorname{sinal}\left(u_{f}\right)\right)
$$

em que $\phi_{D}, \phi_{U}, \phi_{R}$ denotam a variável $\phi$ avaliada nas posições $D, U$, e $R$, respectivamente.

A partir deste ponto, considera-se apenas a face $f=i+1 / 2$ em que a velocidade de convecção é positiva $\left(u_{(i+1 / 2, j)}>0\right)$. Os outros casos seguem procedimentos similares. Como exemplo, considera-se o ponto $(i+1 / 2, j)$, com interface $f=i+1 / 2$; neste caso a interpolação upwind para o fluxo numérico em $(i+1 / 2, j)$ é como segue

$$
\phi_{(i+1 / 2, j)}=\phi_{(i+1 / 2, j)}\left(\phi_{(i+1, j)}, \phi_{(i, j)}, \phi_{(i-1, j)}, \operatorname{sinal}\left(u_{(i+1 / 2, j)}\right)\right)
$$

com os pontos $D=(i+1, j), U=(i, j)$ e $R=(i-1, j)$ dispostos na Figura 3.1(a); caso a velocidade de convecção seja negativa, isto é $u_{(i+1 / 2, j)}<0$, a disposição desses pontos são como ilustrado na Figura 3.1(b).

Como foi visto anteriormente, o valor da variável transportada $\phi$ na interface $f$ é obtido em função dos pontos $D, U$ e $R$ e de maneira upwind, isto é, $\phi_{f}=\phi\left(\phi_{D}, \phi_{U}, \phi_{R}\right.$, sinal $\left.\left(u_{f}\right)\right)$. Com o objetivo de reduzir o número de variáveis envolvidas nessa interpolação, uma transformação é feita de tal forma que a função $\phi=\phi_{f}$ dependa apenas da informação atrasada (upwind). Esta transformação, denotada por $\hat{\phi}_{f}$ e definida como

$$
\hat{\phi}_{f}=\frac{\phi_{f}-\phi_{R}}{\phi_{D}-\phi_{R}}
$$

Esta é a representação da função $\phi_{f}$ em variável normalizada (NV) de Leonard [19]. Note que, como $\hat{\phi}_{D}=1$ e $\hat{\phi}_{R}=0$, a relação (3.4) dependerá apenas de $\hat{\phi}_{U}$. Na sequência apresentam-se esquemas convectivos convencionais em suas representações normalizadas e não normalizadas. 


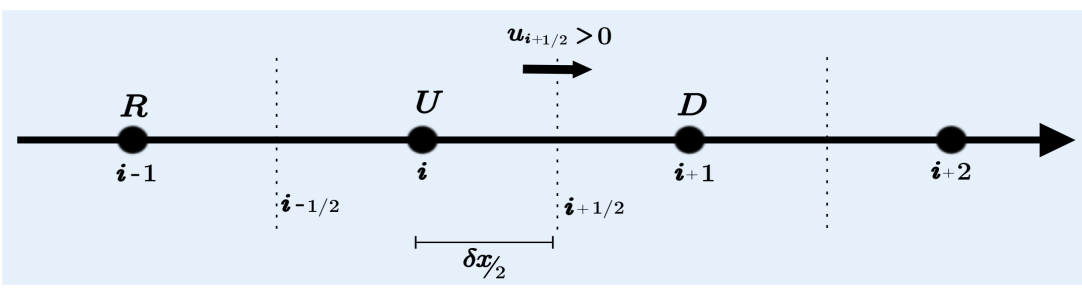

(a) Velocidade de convecção positiva.

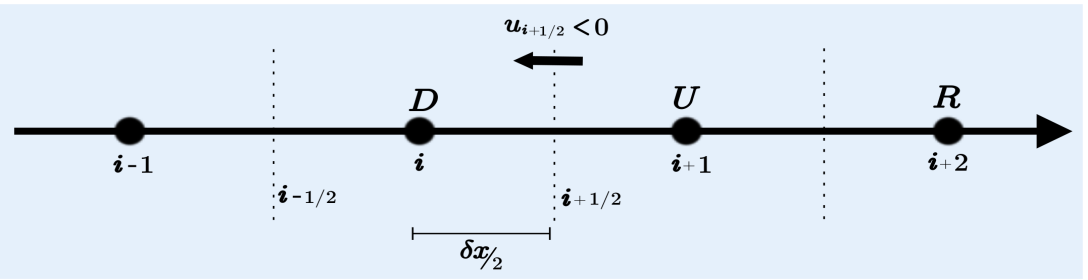

(b) Velocidade de convecção negativa.

Figura 3.1: Definição dos pontos de interpolação $D, U$ e $R$ com respeito à interface $f=i+1 / 2$ e o sinal da velocidade de convecção $u_{(i+1 / 2, j)}$.

O esquema FOU (First-Order Upwind) é um exemplo clássico de esquema convectivo de primeira ordem. Sua representação, na forma não normalizada, é dada por $\phi_{f}=\phi_{(i, j)}=\phi_{U}$, e, na forma normalizada, por $\hat{\phi}_{f}=\hat{\phi}_{U}$. A Figura 3.2 mostra o desempenho desse esquema em várias malhas computacionais com um número de Courant $C F L=0.1$, aplicado ao problema de advecção de uma onda quadrada. Vê-se claramente nesta figura que para se ter uma boa solução numérica com este esquema deve-se utilizar uma malha computacional suficientemente refinada. Observa-se também que o esquema FOU é bastante difusivo, isto é ele suaviza sobremaneira a solução nas regiões próximas às descontinuidades (ver Figura 3.2(a)).

O esquema convectivo QUICK (Quadratic Upstream Interpolation for Convective Kinematics) é outro exemplo convencional de esquemas upwind, representado em forma não normalizada por

$$
\phi_{f}=\frac{1}{2}\left(\phi_{D}+\phi_{U}\right)-\frac{1}{8}\left(\phi_{D}-2 \phi_{U}+\phi_{R}\right)
$$

e na forma normalizada por

$$
\hat{\phi}_{f}=\frac{3}{4} \hat{\phi}_{U}+\frac{3}{8}
$$

Ainda que atinja terceira ordem de precisão (ver [18]), o esquema QUICK possui características indesejáveis em aplicações: as comumente chamadas oscilações não físicas. Essas oscilações podem ser vistas na Figura 3.3, onde apresentam-se as soluções numérica e analítica para o transporte de uma onda quadrada via equação de advecção 1D.

Ambos esquemas (FOU e QUICK) dependem linearmente de $\hat{\phi}_{U}$. Esta dependência é ilustrada na Figura 3.4, em que as curvas mostradas são chamadas de características NV. A região indicada como CBC nesta figura corresponde ao diagrama de variáveis normalizadas CBC (Convection Boundedness Criterion) de Gaskell e Lau [12]. 
(a)

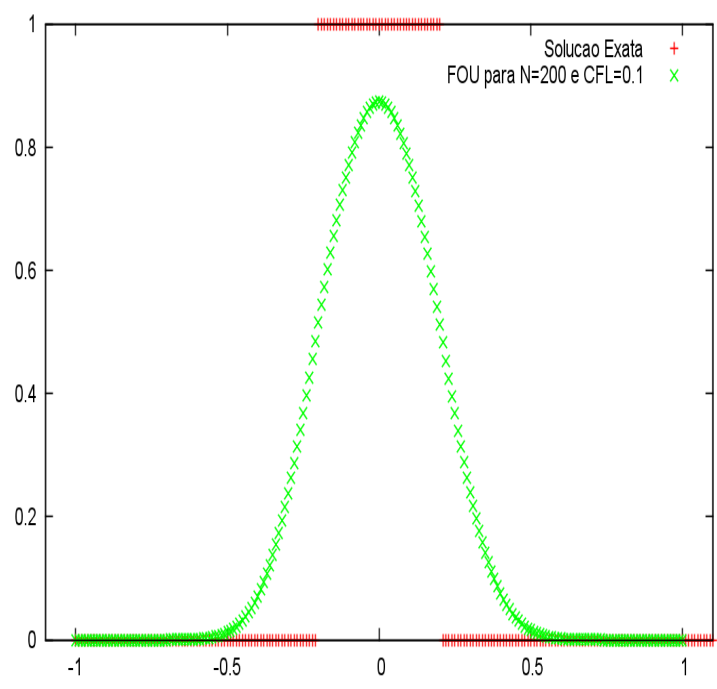

(c)

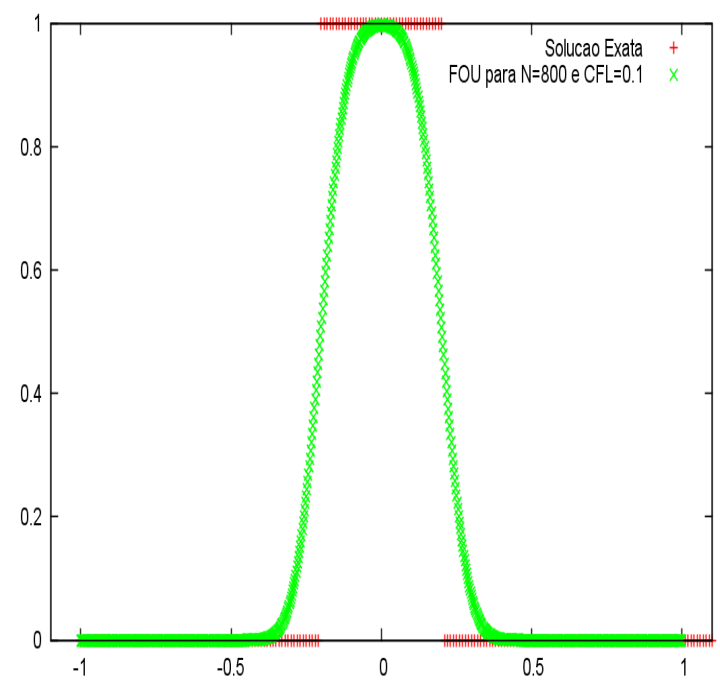

(b)

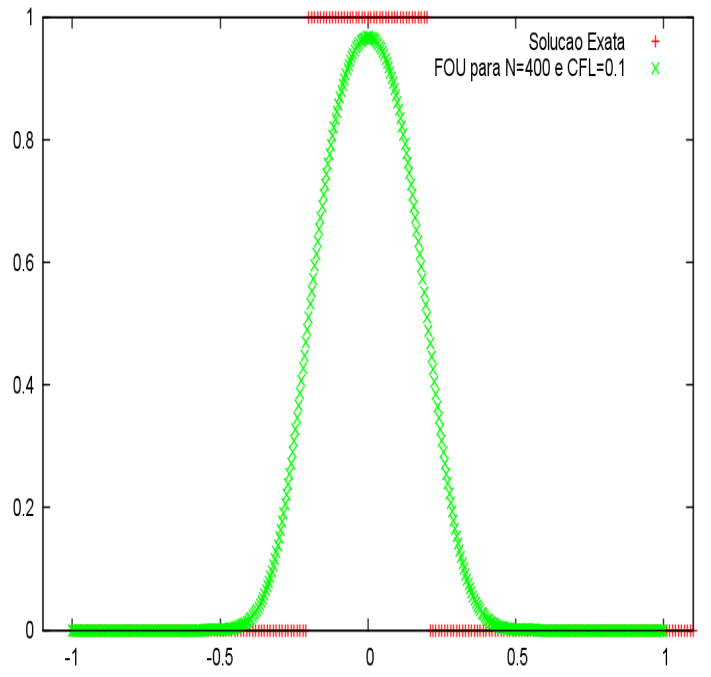

(d)

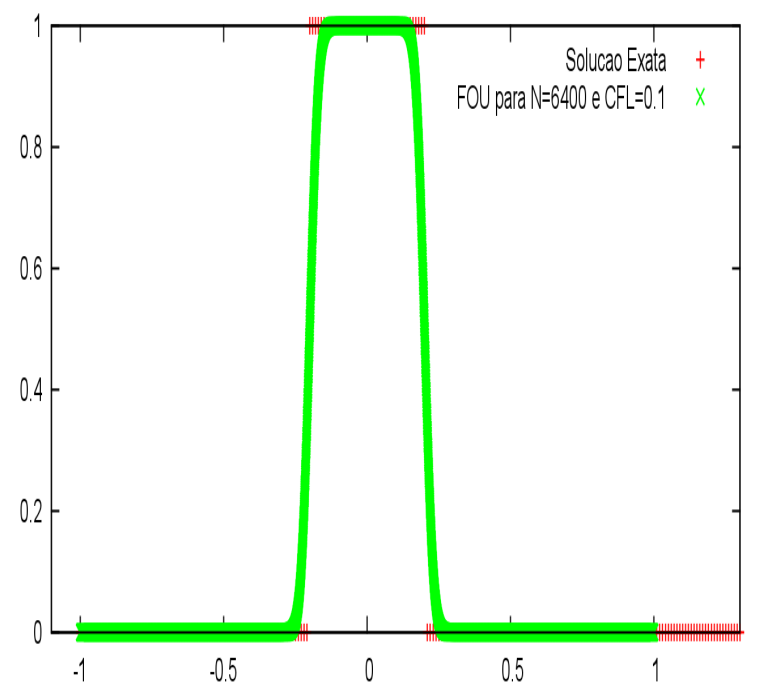

Figura 3.2: Comparações das soluções numéricas em várias malhas (com o esquema FOU) e solução analítica para advecção de um escalar. (a) malha 200; (b) malha 400; (c) malha 800; e (d) malha 6400. 


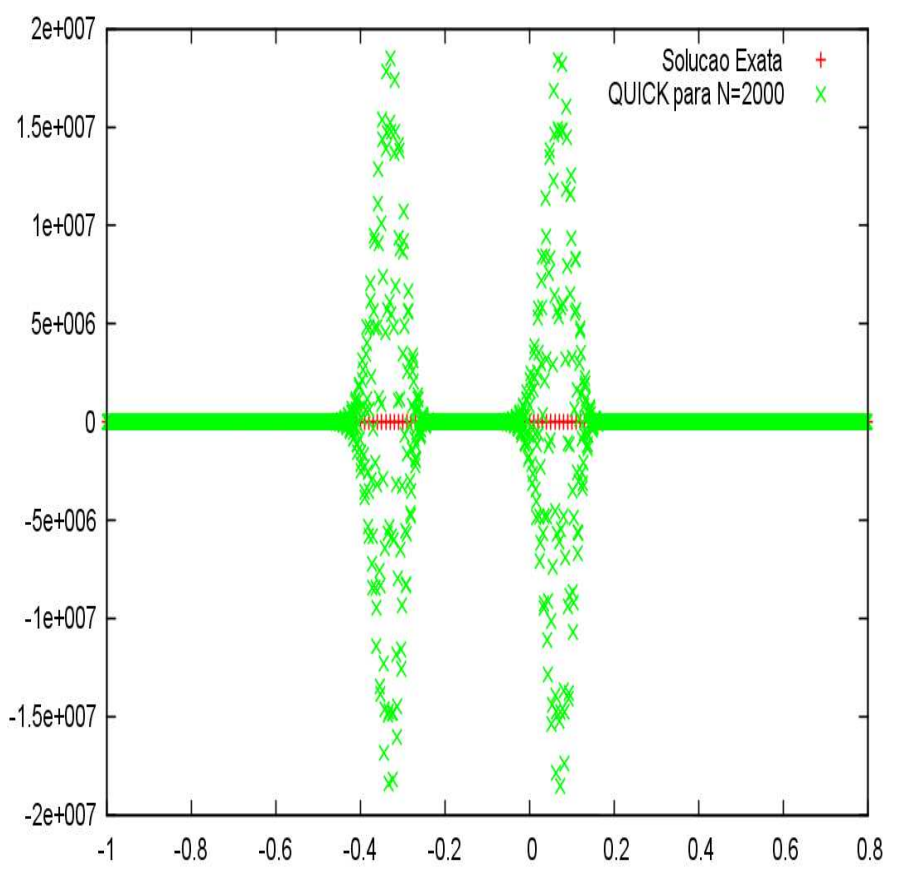

Figura 3.3: Comparação das soluções numérica (com o esquema QUICK) e analítica numa malha computacional de 2000 pontos.

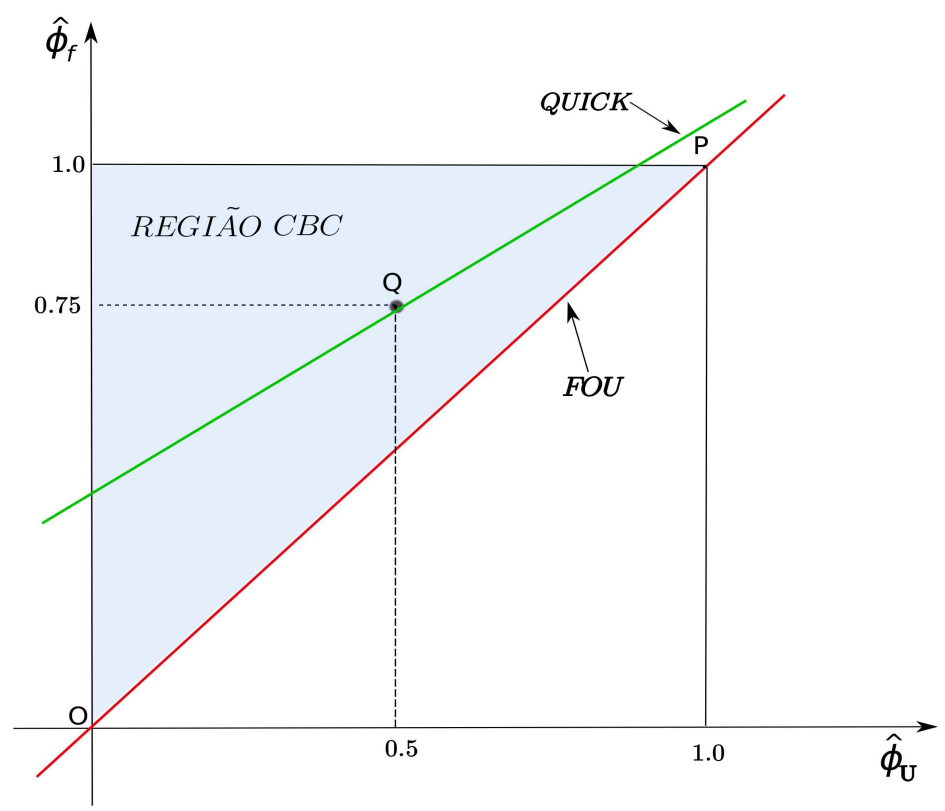

Figura 3.4: Características NV dos esquemas convectivos FOU e QUICK; e diagrama de variáveis normalizadas, mostrando a região hachurada CBC. 
Usando série de Taylor, pode-se mostrar (ver [19]) que, para qualquer esquema baseado em características NV (lineares ou não lineares), valem as seguintes propriedades:

- Passar no ponto $\left(\frac{1}{2}, \frac{3}{4}\right)$ do diagrama NV alcança segunda ordem de precisão local;

- Ter inclinação $\frac{3}{4}$, enquanto passa no ponto $\left(\frac{1}{2}, \frac{3}{4}\right)$, atinje terceira ordem de precisão local.

Leonard observou também que esquemas que passam em $O=(0,0)$ e $P=(1,1)$ são livres de oscilações numéricas. Vê-se claramente que o esquema FOU é de primeira ordem e o esquema QUICK é de terceira ordem. O esquema FOU, apesar da baixa ordem, se comporta de maneira estável, mas suaviza a solução (difusão). O esquema QUICK, por outro lado, sendo de ordem mais alta (precisão melhor), produz oscilações não físicas (dispersão).

Para superar esses defeitos, vários esquemas upwind de alta resolução têm aparecido na literatura tais como SMART (Sharp and Monotonic Algorithm for Realistic Transport) [12], VONOS (Variable-Order Non-Oscillatory Scheme) [47], SHARP (Simple High Accuracy Resolution Program) [20], WACEB (Weighted-Average Coefficient Ensuring Boundedness) [39], CUBISTA (Convergent and Universally Bounded Interpolation Scheme for the Treatment of Advection) [1], entre muito outros. O objetivo principal desses métodos é resolver problemas propensos a oscilações e, ao mesmo tempo, melhorar a precisão local. É importante notar, entretanto, que esses esquemas (alguns deles ao menos), embora funcionando bem em alguns problemas, podem não ser limitados em situações envolvendo descontinuidades (choques) em escoamentos compressíveis (ver, por exemplo, [16], [22]) e/ou escoamentos incompressíveis viscoelásticos com equações constitutivas hiperbólicas (ver, por exemplo, [54]). Lin e Chieng [23] e Lin e Lin [22], por exemplo, observaram que os esquemas SMART e SHARP, embora alcancem alta ordem de convergência, produzem oscilações espúrias em problemas com descontinuidades (como por exemplo, o problema do tubo de choque). Alves et al [1] usando esquemas upwind de alta ordem, fizeram uma série de testes para simular fluidos viscoelásticos e observaram dificuldades de convergência quando a malha era refinada, com uma forte tendência a oscilar.

As características indesejáveis associadas a ambos os esquemas clássicos FOU e QUICK e os problemas de limitação com esquemas de alta resolução citados motivam o estudo de novos esquemas convectivos com boa ordem de precisão $(\geq 2)$, ao mesmo tempo livres de oscilações não físicas, que resolvam bem problemas complexos.

\subsection{Critérios de limitação e exemplos de esquemas upwind}

Durante a simulação computacional de problemas complexos, é imprescindível que as soluções obtidas não ultrapassem os valores físicos do processo que está sendo simulado. Segundo Gaskell e Lau [12], dada uma característica NV contínua (ou contínua por partes) $F: \hat{\phi}_{U} \rightarrow \hat{\phi}_{f}$, o es- 
quema correspondente preserva monotonicidade se e somente se

$$
\begin{cases}\hat{\phi}_{U} \leq F\left(\hat{\phi}_{U}\right) \leq 1, & \text { se } \quad \hat{\phi}_{U} \in[0,1] \\ F\left(\hat{\phi}_{U}\right)=\hat{\phi}_{U}, & \text { se } \hat{\phi}_{U} \notin[0,1]\end{cases}
$$

Este critério de limitação é representado geometricamente na Figura 3.4 e define a região de estabilidade do processo numérico.

Um esquema convectivo upwind pode ser desenvolvido considerando a aproximação geral

$$
\phi_{f}=\phi_{U}+B\left[\left.\left(\frac{\partial \phi}{\partial x}\right)\right|_{f},\left.\left(\frac{\partial \phi}{\partial x}\right)\right|_{g}, \delta x\right]
$$

onde a função $B$ é um termo anti-difusivo. Na literatura, é comum tomar a função $B$ como

$$
B=\left.\frac{1}{2} \delta x\left(\frac{\partial \phi}{\partial x}\right)\right|_{f}
$$

O esquema convectivo upwind (3.10) pode ser então aproximado por

$$
\phi_{f} \approx \phi_{U}+\left.\frac{1}{2} \delta x\left(\frac{\partial \phi}{\partial x}\right)\right|_{f} \approx \phi_{U}+\frac{1}{2}\left(\phi_{D}-\phi_{U}\right)
$$

o qual é propenso à formação de oscilações não físicas (similar ao que foi visto com o uso do esquema QUICK). Para corrigir essas oscilações, introduz-se uma função $\psi$ (chamada limitador de fluxo) na formulação (3.12). Isto permite manter a solução numérica dentro de regiões estáveis, isto é,

$$
\phi_{f} \approx \phi_{U}+\frac{1}{2} \psi\left(r_{f}\right)\left(\phi_{D}-\phi_{U}\right)
$$

onde $r_{f}$ é um sensor de suavidade da solução definido como

$$
r_{f}=r_{(i+1 / 2, j)}=\frac{\left.\left(\frac{\partial \phi}{\partial x}\right)\right|_{g}}{\left.\left(\frac{\partial \phi}{\partial x}\right)\right|_{f}} \approx \frac{\Delta \phi_{2}}{\Delta \phi_{1}}=\frac{\phi_{U}-\phi_{R}}{\phi_{D}-\phi_{U}}=\frac{\phi_{(i, j)}-\phi_{(i-1, j)}}{\phi_{(i+1, j)}-\phi_{(i, j)}}
$$

Alguns exemplos de limitadores são como segue:

- $\psi=0 \rightarrow$ FOU;

- $\psi=1 \rightarrow$ Diferença Central;

- $\psi=r_{f} \rightarrow$ upwind linear. 
Segundo Harten [14], um bom limitador de fluxo é aquele que para $r_{f}<0$ se transforme no esquema FOU e para $r_{f}>0$ esteja restrito às regiões de monotonicidade, apresentando comportamento assintótico nesta região. Esse objetivo pode ser alcançado por meio do critério de limitação TVD (Total Variation Diminishing) de Harten [14] que garante soluções numéricas fisicamente aceitáveis, livres de oscilações e com boa ordem de precisão. Em NV, o esquema upwind (3.13) é escrito como

$$
\hat{\phi}_{f} \approx \hat{\phi}_{U}+\frac{1}{2} \psi\left(r_{f}\right)\left(1-\hat{\phi}_{U}\right)
$$

$\mathrm{ou}$

$$
\hat{\phi}_{(1+1 / 2, j)} \approx \hat{\phi}_{(i, j)}+\frac{1}{2} \psi\left(r_{f}\right)\left(1-\hat{\phi}_{(i, j)}\right)
$$

onde

$$
r_{f}=r_{(i+1 / 2, j)}=\frac{\hat{\phi}_{U}}{1-\hat{\phi}_{U}}=\frac{\hat{\phi}_{(i, j)}}{1-\hat{\phi}_{(i, j)}} .
$$

Como comentado anteriormente, o parâmetro $r_{f}$ funciona como um detector de suavidade da solução. Com base nos valores de $r_{f}$, várias regiões de interesse podem ser identificadas no plano $\psi\left(r_{f}\right) \perp r_{f}$. A reta $r_{f}=0$, como mostrado na Figura 3.5, separa regiões de oscilações ou extremos $\left(r_{f}<0\right)$ de regiões de soluções monotônicas $\left(r_{f}>0\right)$. Dentro dessas últimas, $r_{f} \approx 1$ corresponde a regiões de soluções suaves $\left(r_{f}=1\right.$ - variação linear). Regiões de altas curvaturas podem ser identificadas para $r_{f} \leq 0$ e $r_{f}<<1$ (curvatura negativa) ou $r_{f}>>1$ (curvatura positiva).

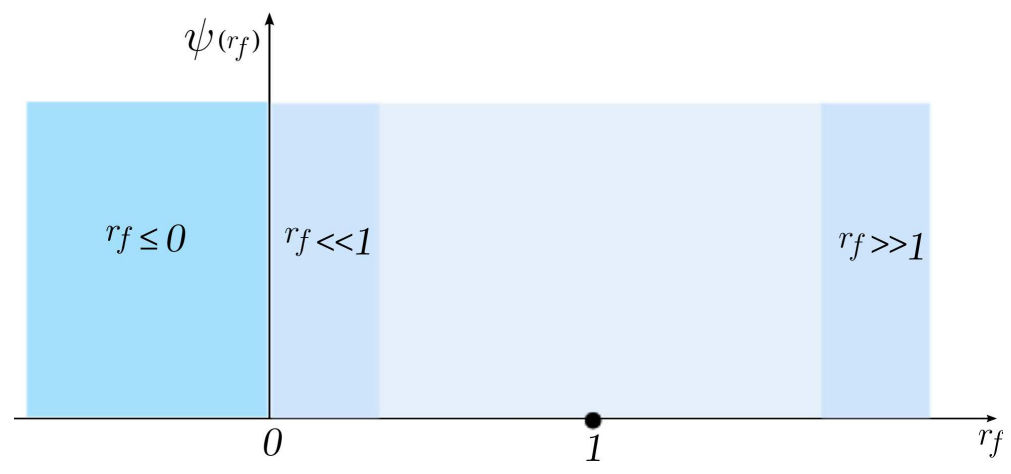

Figura 3.5: Plano $\psi(r) \perp r$ mostrando a região de extremos $\left(r_{f} \leq 0\right)$, a região de monotonicidade (vizinhança de $r_{f}=1$ ) e as regiões de alta curvatura $\left(r_{f}>>1\right.$ e $r_{f}<<1$ ).

A variação total $(T V)$ no nível de tempo $t_{j}=j \delta_{t}$ é definida como

$$
T V\left(\phi\left(t_{j}\right)\right)=T V\left(\phi^{j}\right)=\sum_{i}\left|\phi_{(i+1, j)}-\phi_{(i, j)}\right|
$$

Um esquema é TVD se 


$$
T V\left(\phi^{j+1}\right) \leq T V\left(\phi^{j}\right)
$$

O termo Diminishing refere-se ao fato de que a variação total num dado tempo pode ser no máximo a variação total no tempo anterior, não crescente com a marcha no tempo. A propriedade TVD é uma propriedade global de um esquema advectivo e previne a amplificação espúria de extremos. Harten[14] provou que um perfil (inicialmente) monotônico $\phi$ permanece monotônico após ser advectado por um esquema TVD. Entretanto, a propriedade TVD não exclui a possibilidade de que um extremo possa crescer enquanto outro diminui.

No caso de problemas com soluções descontínuas, faz-se necessário empregar métodos monotônicos para que não haja oscilações nas vizinhanças das descontinuidades. LeVeque [21] demonstrou que se o esquema é TVD, ele preserva monotonicidade. Sweby [41] aplicou o conceito de Harten [14] aos limitadores de fluxo, e propôs a restrição

$$
\begin{cases}0 \leq \psi\left(r_{f}\right) \leq \min \left(2 r_{f}, 2\right), & \text { se } \quad r_{f} \geq 0 \\ \psi\left(r_{f}\right)=0, & \text { se } \quad r_{f} \leq 0\end{cases}
$$

a qual define a comumente chamada região TVD (ver Figura 3.6) para os limitadores de fluxo.

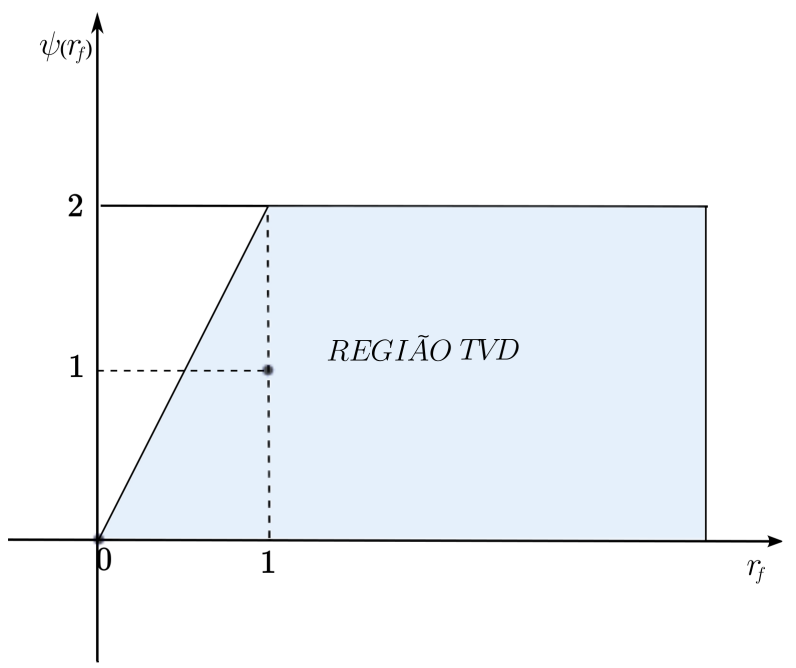

Figura 3.6: Região TVD de Sweby.

É mostrado a seguir que qualquer limitador de fluxo $\psi$ que satisfaz $\psi(1)=1$ (princípio de Sweby [41]), e que faz com que (3.13) seja de segunda ordem de precisão deve satisfazer a seguinte condição de equivalência:

$$
\left.\frac{d \psi}{d r_{f}}\right|_{r_{f}=1}=\frac{1}{4} \Leftrightarrow \text { o esquema (3.13) tem terceira ordem de preciso local. }
$$

De fato, pois: 
$(\Leftarrow)$ Levando os desenvolvimentos, em série de Taylor em torno do ponto $(i+1 / 2, j)$, $\operatorname{de} \phi_{(i-1, j)}=\phi_{([i+1 / 2]-3 / 2, j)}, \phi_{(i, j)}=\phi_{([i+1 / 2]-1 / 2, j)}$ e $\phi_{(i+1, j)}=\phi_{([i+1 / 2]+1 / 2, j)}$, mais $\psi\left(r_{f}\right) \approx$ $\psi(1)+\left.\left(r_{f}-1\right) \frac{d \psi}{d r_{f}}\right|_{r_{f}=1}$, na expressão $(3.13)$, resulta $\left.\frac{d \psi}{d r_{f}}\right|_{r_{f}=1}=\frac{1}{4}$.

$(\Rightarrow)$ A derivada da expressão (3.16) em relação a $\hat{\phi}_{(i, j)}$ dá

$$
\begin{aligned}
& \frac{d \hat{\phi}_{(i+1 / 2, j)}}{d \hat{\phi}_{(i, j)}}=1+\frac{1}{2} \frac{d}{d \hat{\phi}_{(i, j)}}\left[\psi\left(r_{f(i+1 / 2, j)}\right)\left(1-\hat{\phi}_{(i, j)}\right)\right] \\
& =1+\frac{1}{2}\left[\frac{d \psi}{d r_{f(i+1 / 2, j)}} \cdot \frac{d r_{f(i+1 / 2, j)}}{d \hat{\phi}_{(i, j)}} \cdot\left(1-\hat{\phi}_{(i, j)}\right)-\psi\left(r_{f(i+1 / 2, j)}\right)\right] \\
& =1+\frac{1}{2}\left[\frac{d \psi}{d r_{f(i+1 / 2, j)}} \cdot \frac{1}{\left(1-\hat{\phi}_{(i, j)}\right)^{2}} \cdot\left(1-\hat{\phi}_{(i, j)}\right)-\psi\left(r_{f(i+1 / 2, j)}\right)\right] \text {. }
\end{aligned}
$$

Avaliando a expressão $(3.22)$ em $\hat{\phi}_{(i, j)}=\frac{1}{2}$ (o que equivale a $r_{f(i+1 / 2, j)}=1$ ) obtém-se

$$
\frac{d \hat{\phi}_{(i+1 / 2, j)}}{d \hat{\phi}_{(i, j)}}=\frac{3}{4} .
$$

Essa é a condição de Leonard [19] para esquemas upwind alcançarem terceira ordem de precisão.

Utilizando-se da idéia de monotonicidade, Sweby [41] definiu o princípio de monotonicidade para limitadores de fluxo, em que

$$
\psi^{\prime}\left(r_{f}\right)=2 \quad \text { se } \quad r_{f} \rightarrow 0
$$

Combinando as relações entre $\phi_{U}$ e $\phi_{f}$, e considerando as relações (3.12), (3.14), (3.15), obtém-se a região para as características upwind NV (ver Figura 3.7) que satisfazem a restrição TVD, isto é

$$
\begin{cases}\hat{\phi}_{U} \leq \hat{\phi}_{f} \leq \min \left(1,2 \hat{\phi}_{U}\right), & \text { se } 0<\hat{\phi}_{U}<1 \\ \hat{\phi}_{f}=\hat{\phi}_{U}, & \text { se } \hat{\phi}_{U} \leq 0 \text { ou } \hat{\phi}_{U} \geq 1\end{cases}
$$

Os esquemas apresentados a seguir são exemplos representativos de esquemas TVD de alta resolução diferenciáveis, e que serão utilizados para comparações com os esquemas upwind TVD desenvolvidos neste trabalho.

- Esquema de van Albada [46]

$$
\hat{\phi}_{f}= \begin{cases}\frac{4 \hat{\phi}_{U}^{3}-5 \hat{\phi}_{U}^{2}+3 \hat{\phi}_{U}}{4 \hat{\phi}_{U}^{2}-4 \hat{\phi}_{U}+2}, & \text { se } 0 \leq \hat{\phi}_{U} \leq 1 \\ \hat{\phi}_{U}, & \text { se } \quad \hat{\phi}_{U} \notin[0,1]\end{cases}
$$




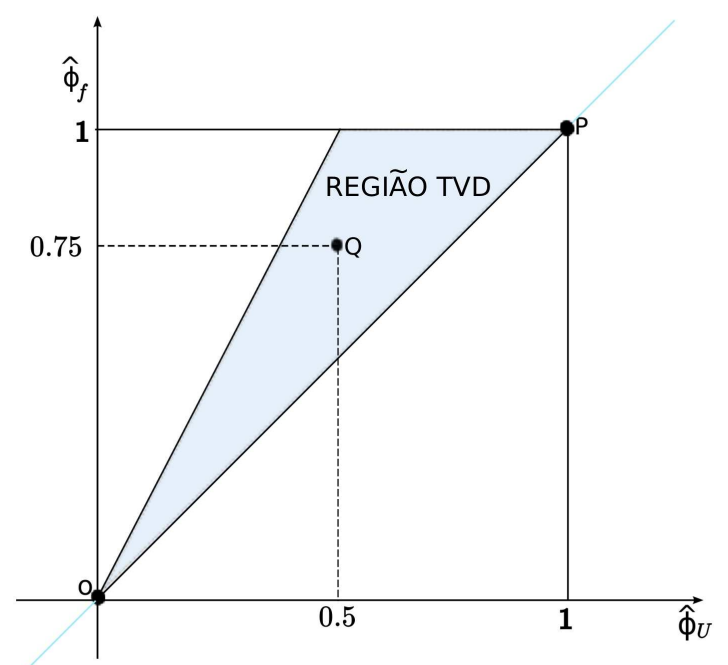

Figura 3.7: Região TVD em variáveis normalizadas.

cujo limitador de fluxo correspondente é

$$
\psi\left(r_{f}\right)=\frac{r_{f}^{2}+r_{f}}{r_{f}^{2}+1}
$$

- Esquema EPUS de Corrêa [7]

$$
\hat{\phi}_{f}= \begin{cases}-4(\lambda-24) \hat{\phi}_{U}^{8}+16(\lambda-23) \hat{\phi}_{U}^{7}+(528-25 \lambda) \hat{\phi}_{U}^{6}+ & \\ (19 \lambda-336) \hat{\phi}_{U}^{5}+(80-7 \lambda) \hat{\phi}_{U}^{4}+\lambda \hat{\phi}_{U}^{3}+\hat{\phi}_{U}, & \text { se } \hat{\phi}_{U} \in[0,1], \\ \hat{\phi}_{U}, & \text { se } \quad \hat{\phi}_{U} \notin[0,1] .\end{cases}
$$

$\lambda \in[16,95]$, cujo limitador de fluxo correspondente é

$$
\psi\left(r_{f}\right)=\max \left\{0, \frac{0.5\left(\left|r_{f}\right|+r_{f}\right)(2 \lambda-32) r_{f}^{4}+(160-4 \lambda) r_{f}^{3}+2 \lambda r_{f}^{2}}{\left(1+\left|r_{f}\right|\right)^{7}}\right\} .
$$

Muito embora os limitadores van Albada e EPUS (e muitos outros esquemas upwind presentes na literatura) sejam diferenciáveis, na prática (principalmente em problemas incompressíveis) o usuário pode enfrentar dificuldades com o uso desses esquemas no que diz respeito à convergência do processo numérico global. Segundo Venkatakrishnan [48] a razão para esse comportamento parece ser que limitadores desse tipo atuam nas oscilações em escalas pequenas nas regiões suaves, introduzindo assim efeitos não lineares. Isso faz com que o resíduo sofra 
do incômodo problema de estagnação. Com o objetivo de evitar esse problema de estagnação, utiliza-se a técnica dos mínimos quadrados ponderados descrita a seguir.

Uma forma de estimar o limitador de fluxo $\psi\left(r_{f}\right)$ dado em (3.13) pode ser feita através da técnica de minimização de erros denominada mínimos quadrados ponderados. Tomando-se a molécula computacional de três pontos dada na Figura 3.1, constrói-se o sistema indeterminado através do truncamento da série de Taylor. Por simplicidade, restringindo-se ao caso em que a velocidade $v_{f}$ é positiva (o caso negativo é análogo), e o domínio é 1D (a extensão para outras dimensões é trivial, e apenas altera a dimensão do sistema), tem-se:

$$
\left\{\begin{array}{l}
\phi_{D}=\phi_{U}+\left.\delta_{x}\left(\frac{d \phi}{d_{x}}\right)\right|_{U} \Rightarrow \phi_{D}-\phi_{U}=\Delta \phi_{1}=\left.\delta_{x}\left(\frac{d \phi}{d_{x}}\right)\right|_{U} \\
\phi_{R}=\phi_{U}-\left.\delta_{x}\left(\frac{d \phi}{d_{x}}\right)\right|_{U} \Rightarrow \phi_{U}-\phi_{R}=\Delta \phi_{2}=\left.\delta_{x}\left(\frac{d \phi}{d_{x}}\right)\right|_{U}
\end{array}\right.
$$

Em forma matricial o sistema (3.28) torna-se

$$
\Delta \phi=S d_{\phi}
$$

onde $\Delta \phi=\left[\Delta \phi_{1}, \Delta \phi_{2}\right]^{T}, S=\left[\delta_{x}, \delta_{x}\right]^{T}$ e $d_{\phi}=\left.\left(\frac{d \phi}{d_{x}}\right)\right|_{U}$. Admitindo-se que exista um $b$ ótimo, tal que $d_{\phi}=b$, estima-se este valor por meio da técnica de mínimos quadrados. Neste caso o erro $E(b)$ é dado por

$$
E(b)=\sum_{j=1}^{2}\left(\Delta \phi_{j}-S_{j} b\right)^{2}
$$

de maneira que o processo de minimização leva a

$$
S^{T} \Delta \phi=S^{T} S b
$$

cuja melhor aproximação é

$$
b=\left(S^{T} S\right)^{-1} S^{T} \Delta \phi
$$

A forma ponderada do limitador de fluxo (chamada técnica dos mínimos quadrados ponderados) é obtida inserindo-se a matriz $W=\left[w_{1}, w_{2}\right]^{T}$, com $w_{1}$ e $w_{2}$ pesos prescritos, na expressão (3.32), isto é

$$
b=\left(S^{T} W S\right)^{-1} S^{T} W \Delta \phi
$$

e o diferencial $d_{\phi}$ é calculado por meio da expressão

$$
d_{\phi}=\frac{\Delta \phi_{1} w_{1}+\Delta \phi_{2} w_{2}}{\left(w_{1}+w_{2}\right) \delta_{x}}
$$

Desta forma, da expansão em série de Taylor de $\phi$ em torno da face $f=i+\frac{1}{2}$, obtém-se

$$
\phi_{f}=\phi_{(i+1 / 2, j)}=\phi_{U}+\frac{1}{2} \frac{\Delta \phi_{1} w_{1}+\Delta \phi_{2} w_{2}}{\left(w_{1}+w_{2}\right)} .
$$


O limitador resultante é então dado por

$$
\psi\left(r_{f}\right)=\text { Pond } \cdot \frac{\Delta \phi_{1} w_{1}+\Delta \phi_{2} w_{2}}{\left(w_{1}+w_{2}\right) \Delta \phi_{1}},
$$

onde Pond é uma constante arbitrária. É comum introduzir um parâmetro pequeno $\epsilon$ na expressão (3.36) (veja, por exemplo, [48] ou [26]) para prevenir indeterminação em regiões de altos gradientes; e o resultado final do limitador de fluxo é

$$
\psi\left(r_{f}\right)=\text { Pond } \cdot \frac{\Delta \phi_{1}\left(w_{1}+\epsilon\right)+\Delta \phi_{2}\left(w_{2}+\epsilon\right)}{\left(w_{1}+w_{2}+2 \epsilon\right) \Delta \phi_{1}} .
$$




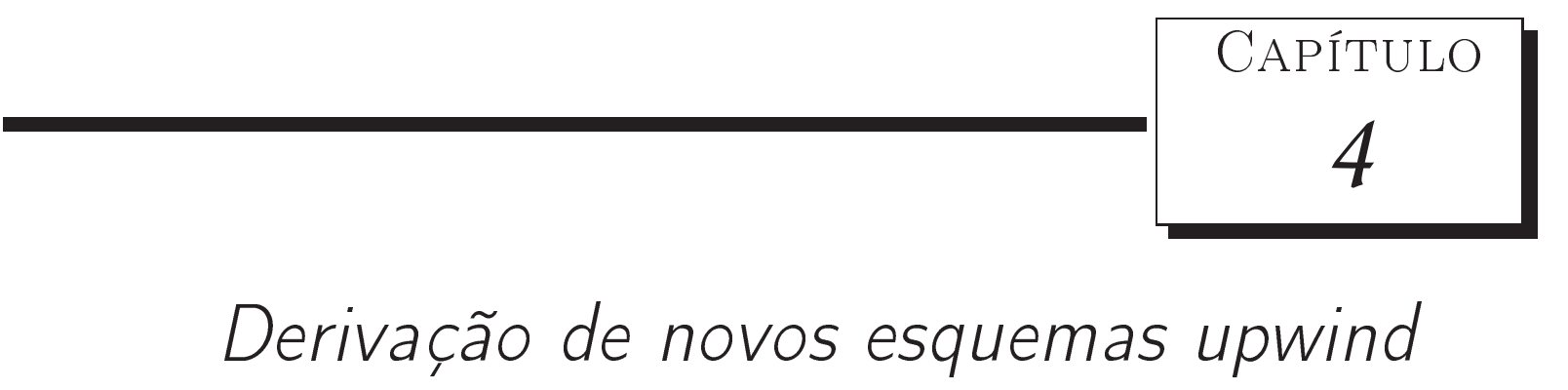

Neste capítulo apresentam-se novos esquemas upwind do tipo funções racionais, cujos desenvolvimentos são baseados nas condições apresentadas no capítulo 3. Os esquemas estão agrupados em uma família, denominada FUS-RF (Family of Upwind Scheme via Rational Functions). Em particular, seis representantes desta família são apresentados neste estudo, os quais são denominados Esquema 1, Esquema 2, Esquema 3, Esquema 4, Esquema 5 e Esquema 6. O Esquema 1 é desenvolvido de maneira usual, isto é, com base nos critérios de estabilidade TVD/CBC. Os Esquemas 2 a 6 são construídos utilizando-se da aproximação dos gradientes via técnica de minimização mínimos quadrados ponderada.

\subsection{Derivação de esquemas upwind via forma usual: Es- quema 1}

A ideia por trás do desenvolvimento do Esquema 1 é derivar uma função racional como inversa de um polinômio de grau cinco em $\hat{\phi}_{U}$, subtraida uma constante $\beta$, a qual passa pelos pontos $(0,0),(1,1)$ e $\left(\frac{1}{2}, \frac{3}{4}\right)$ com inclinação $\frac{3}{4}$ em $\left(\frac{1}{2}, \frac{3}{4}\right)$, ou seja

$$
\hat{\phi}_{f}=\left\{\begin{array}{lll}
\frac{1}{\alpha_{0}+\alpha_{1} \hat{\phi}_{U}+\alpha_{2} \hat{\phi}_{U}^{2}+\alpha_{3} \hat{\phi}_{U}^{3}+\alpha_{4} \hat{\phi}_{U}^{4}+\alpha_{5} \hat{\phi}_{U}^{5}}-\beta, & \text { se } \quad 0 \leq \hat{\phi}_{U} \leq 1, \\
\hat{\phi}_{U}, & \text { se } \quad \hat{\phi}_{U} \notin[0,1]
\end{array}\right.
$$

em que $\alpha_{i}, i=0, \cdots, 5$ são constantes a serem determinadas. A inclusão do parâmetro $\beta$ em (4.1) é imprescindível para que o esquema passe na origem. Impondo as quatro condições acima, mais a imposição de que o esquema (4.1) tenha a mesma inclinação do FOU em $(0,0)$, $(1,1)$ (classe $C^{1}$ ), obtém-se o sistema linear de equações

- $\hat{\phi}_{f}(0)=0 \quad \Rightarrow \quad \alpha_{0}=\frac{1}{\beta}$; 
- $\hat{\phi}_{f}(1)=1 \quad \Rightarrow \quad \alpha_{0}+\alpha_{1}+\alpha_{2}+\alpha_{3}+\alpha_{4}+\alpha_{5}=\frac{1}{1+\beta}$;

- $\hat{\phi}_{f}\left(\frac{1}{2}\right)=\frac{3}{4} \quad \Rightarrow \quad \alpha_{0}+\frac{1}{2} \alpha_{1}+\frac{1}{4} \alpha_{2}+\frac{1}{8} \alpha_{3}+\frac{1}{16} \alpha_{4}+\frac{1}{32} \alpha_{5}=\frac{4}{3+4 \beta}$;

- $\hat{\phi}_{f}^{\prime}\left(\frac{1}{2}\right)=\frac{3}{4} \quad \Rightarrow \quad-4 \alpha_{1}-4 \alpha_{2}-3 \alpha_{3}-2 \alpha_{4}-\frac{5}{4} \alpha_{5}=3\left(\frac{4}{3+4 \beta}\right)^{2}$;

- $\hat{\phi}_{f}^{\prime}(0)=1 \quad \Rightarrow \quad \alpha_{1}=-\left(\frac{1}{\beta}\right)^{2}$;

- $\hat{\phi}_{f}^{\prime}(1)=1 \quad \Rightarrow \quad-\alpha_{1}-2 \alpha_{2}-\alpha_{3}-4 \alpha_{4}-5 \alpha_{5}=\left(\frac{1}{1+\beta}\right)^{2}$.

A imposição de diferenciabilidade nos pontos $(0,0)$ e $(1,1)$ tem o propósito de evitar problemas de convergência do método numérico global em malhas grosseiras, em analogia ao que foi proposto por Lin e Chieng [23]. Definindo

$$
\lambda_{1}=\frac{1}{\beta}, \quad \lambda_{2}=\frac{1}{1+\beta}, \quad \lambda_{3}=\frac{4}{3+4 \beta},
$$

o sistema anterior torna-se

$$
\left[\begin{array}{cccccc}
1 & 0 & 0 & 0 & 0 & 0 \\
0 & -1 & 0 & 0 & 0 & 0 \\
1 & 1 & 1 & 1 & 1 & 1 \\
1 & \frac{1}{2} & \frac{1}{4} & \frac{1}{8} & \frac{1}{16} & \frac{1}{32} \\
0 & -1 & -2 & -3 & -4 & -5 \\
0 & -4 & -4 & -3 & -2 & -\frac{5}{4}
\end{array}\right]\left[\begin{array}{l}
\alpha_{0} \\
\alpha_{1} \\
\alpha_{2} \\
\alpha_{3} \\
\alpha_{4} \\
\alpha_{5}
\end{array}\right]=\left[\begin{array}{c}
\lambda_{1} \\
\lambda_{1}^{2} \\
\lambda_{2} \\
\lambda_{3} \\
\lambda_{2}^{2} \\
3 \lambda_{3}^{2}
\end{array}\right] .
$$

Resolvendo este sistema via MAPLE tem-se

$$
\begin{aligned}
& \alpha_{0}=\lambda_{1}, \\
& \alpha_{1}=-\left(\lambda_{1}\right)^{2}, \\
& \alpha_{2}=6\left(\lambda_{1}\right)^{2}-23 \lambda_{1}+\left(\lambda_{2}\right)^{2}+7 \lambda_{2}+6\left(\lambda_{3}\right)^{2}+16 \lambda_{3}, \\
& \alpha_{3}=-13\left(\lambda_{1}\right)^{2}+66 \lambda_{1}-5\left(\lambda_{2}\right)^{2}-34 \lambda_{2}-24\left(\lambda_{3}\right)^{2}-32 \lambda_{3}, \\
& \alpha_{4}=12\left(\lambda_{1}\right)^{2}-68 \lambda_{1}+8\left(\lambda_{2}\right)^{2}+52 \lambda_{2}+30\left(\lambda_{3}\right)^{2}+16 \lambda_{3}, \\
& \alpha_{5}=-4\left(\lambda_{1}\right)^{2}+24 \lambda_{1}-4\left(\lambda_{2}\right)^{2}-24 \lambda_{2}-12\left(\lambda_{3}\right)^{2},
\end{aligned}
$$

que em variáveis normalizadas é dado por

$$
\hat{\phi}_{f}=\left\{\begin{array}{c}
{\left[\lambda_{1}-\lambda_{1}^{2} \hat{\phi}_{U}+\left\{6 \lambda_{1}^{2}-23 \lambda_{1}+\lambda_{2}^{2}+7 \lambda_{2}+6 \lambda_{3}^{2}+16 \lambda_{3}\right\} \hat{\phi}_{U}^{2}+\right.} \\
\left\{-13 \lambda_{1}^{2}+66 \lambda_{1}-5 \lambda_{2}^{2}-34 \lambda_{2}-24 \lambda_{3}^{2}-32 \lambda_{3}\right\} \hat{\phi}_{U}^{3}+ \\
\left\{12 \lambda_{1}^{2}-68 \lambda_{1}+8 \lambda_{2}^{2}+52 \lambda_{2}+30 \lambda_{3}^{2}+16 \lambda_{3}\right\} \hat{\phi}_{U}^{4}+ \\
\left.\left\{-4 \lambda_{1}^{2}+24 \lambda_{1}-4 \lambda_{2}^{2}-24 \lambda_{2}-12 \lambda_{3}^{2}\right\} \hat{\phi}_{U}^{5}\right]^{-1}, \quad \text { se } 0 \leq \hat{\phi}_{U} \leq 1, \\
\hat{\phi}_{U}, \quad \text { se } \hat{\phi}_{U} \notin[0,1] .
\end{array}\right.
$$


Este esquema é mostrado na Figura 4.1 para vários valores de $\beta$.

O limitador de fluxo correspondente para o Esquema 1 é obtido de (3.15), isto é

$$
\psi\left(r_{f}\right) \approx 2 \frac{\hat{\phi}_{f}-\hat{\phi}_{U}}{1-\hat{\phi}_{U}}
$$

Da expressão (4.5), escreve-se $\psi\left(r_{f}\right)$ como

$$
\psi\left(r_{f}\right)=\frac{a}{b}
$$

em que

$$
\begin{array}{r}
a=-2 \alpha_{5} \hat{\phi}_{u}^{6}+\left(-2 \alpha_{4}-2 \beta \alpha_{5}\right) \hat{\phi}_{u}^{5}+\left(-2 \alpha_{3}-2 \beta \alpha_{4}\right) \hat{\phi}_{u}^{4}+\left(-2 \alpha_{2}-2 \beta \alpha_{3}\right) \hat{\phi}_{u}^{3}+ \\
\left(-2 \alpha_{1}-2 \beta \alpha_{2}\right) \hat{\phi}_{u}^{2}+\left(-2 \alpha_{0}-2 \beta \alpha_{1}\right) \hat{\phi}_{u}-2 \beta \alpha_{0}+2, \\
b=-\alpha_{5} \hat{\phi}_{u}^{6}+\left(-\alpha_{4}+\alpha_{5}\right) \hat{\phi}_{u}^{5}+\left(-\alpha_{3}+\alpha_{4}\right) \hat{\phi}_{u}^{4}+\left(-\alpha_{1}+\alpha_{2}\right) \hat{\phi}_{u}^{2}+ \\
\left(-\alpha_{2}+\alpha_{3}\right) \hat{\phi}_{u}^{3}+\left(-\alpha_{0}+\alpha_{1}\right) \hat{\phi}_{u}+\alpha_{0} .
\end{array}
$$

Observando que

$$
\hat{\phi}_{U}=\hat{\phi}_{U}\left(r_{f}\right)=\frac{r_{f}}{1+r_{f}}
$$

o limitador de fluxo do Esquema 1 torna-se

$$
\psi\left(r_{f}\right)=\frac{\gamma_{6} r_{f}^{6}+\gamma_{5} r_{f}^{5}+\gamma_{4} r_{f}^{4}+\gamma_{3} r_{f}^{3}+\gamma_{2} r_{f}^{2}+\gamma_{1} r_{f}+\left(2-2 \beta \alpha_{0}\right)}{c},
$$

em que $c$ é dado por

$$
\begin{array}{r}
c=\left(\alpha_{0}+\alpha_{1}+\alpha_{2}+\alpha_{3}+\alpha_{4}+\alpha_{5}\right) r_{f}^{5}+\left(5 \alpha_{0}+4 \alpha_{1}+3 \alpha_{2}+2 \alpha_{3}+\alpha_{4}\right) r_{f}^{4}+ \\
\left(10 \alpha_{0}+6 \alpha_{1}+3 \alpha_{2}+\alpha_{3}\right) r_{f}^{3}+\left(10 \alpha_{0}+4 \alpha_{1}+3 \alpha_{2}\right) r_{f}^{2}+\left(5 \alpha_{0}+\alpha_{1}\right) r_{f}+\alpha_{0},
\end{array}
$$

e $\gamma_{i}, i=1, \cdots, 6$ por 


$$
\begin{aligned}
& \gamma_{1}=(-2-12 \beta) \alpha_{0}+(-2 \beta) \alpha_{1}+12, \\
& \gamma_{2}=(-10-30 \beta) \alpha_{0}+(-2-10 \beta) \alpha_{1}+(-2 \beta) \alpha_{2}+30, \\
& \gamma_{3}=(-20-40 \beta) \alpha_{0}+(-8-20 \beta) \alpha_{1}+(-2-8 \beta) \alpha_{2}+(-2 \beta) \alpha_{3}+40, \\
& \gamma_{4}=(-30-20 \beta) \alpha_{0}+(-12-20 \beta) \alpha_{1}+(-6-12 \beta) \alpha_{2}+(-2-6 \beta) \alpha_{3}+(-2 \beta) \alpha_{4}+30, \\
& \gamma_{5}=(-10-12 \beta) \alpha_{0}+(-8-10 \beta) \alpha_{1}+(-6-8 \beta) \alpha_{2}+(-4-6 \beta) \alpha_{3}+(-2-4 \beta) \alpha_{4}+(-2 \beta) \alpha_{5}+12, \\
& \gamma_{6}=(-2-2 \beta) \alpha_{0}+(-2-2 \beta) \alpha_{1}+(-2-2 \beta) \alpha_{2}+(-2-2 \beta) \alpha_{3}+(-2-2 \beta) \alpha_{4}+(-2-2 \beta) \alpha_{5}+2 .
\end{aligned}
$$

Nota-se que a equação para $\psi\left(r_{f}\right)$ é válida para $r_{f} \geq 0$, uma vez que de (4.1), tem-se $\hat{\phi}_{U} \in[0,1]$ e, desta maneira

$$
\hat{\phi}_{U}=\frac{r_{f}}{1+r_{f}} \quad \Rightarrow \quad 0 \leq \frac{r_{f}}{1+r_{f}} \leq 1 \quad \Rightarrow \quad r_{f} \geq 0
$$

Por outro lado, se $\hat{\phi}_{U} \notin[0,1]$, então $\hat{\phi}_{f}=\hat{\phi}_{U}$ e, neste caso, tem-se $\psi\left(r_{f}\right)=0$. Assim, o limitador de fluxo é dado por

$$
\psi\left(r_{f}\right)=\frac{0.5\left(\left|r_{f}\right|+r_{f}\right)\left[\gamma_{6} r_{f}^{5}+\gamma_{5} r_{f}^{4}+\gamma_{4} r_{f}^{3}+\gamma_{3} r_{f}^{2}+\gamma_{2} r_{f}+\gamma_{1}+\left(2-2 \beta \alpha_{0}\right) r_{f}^{-1}\right]}{c}
$$

O Esquema 1 satisfaz as restrições TVD (3.24) para $\beta \in[0.1545, \infty]$. Observa-se também que o valor $\beta=0.1545$ fornece um limitante superior para as características NV, enquanto $\beta=0.4045$ fornece um limitante inferior para as características (ver Figura 4.1). No estudo apresentado neste trabalho, tomou-se 10 valores do parâmetro $\beta \in[0.1545,0.4045]$. Vê-se também que em virtude do principio de Sweby e de (3.21), o Esquema 1 pode alcançar até terceira ordem de precisão; de fato, pois

$$
\psi(1)=\frac{(3+4 \beta)^{2}-\left(16 \beta^{2}+20 \beta+6\right)}{3+4 \beta}=1, \quad \forall \beta,
$$

$\mathrm{e}$

$$
\psi^{\prime}(1)=\frac{27+108 \beta+144 \beta^{2}+64 \beta^{3}}{108+432 \beta+576 \beta^{2}+256 \beta^{3}}=\frac{1}{4}, \quad \forall \beta .
$$

Na Figura 4.2, apresenta-se o limitador de fluxo do Esquema 1 no plano $\psi_{f} \perp r_{f}$ para vários valores do parâmetro $\beta$. 


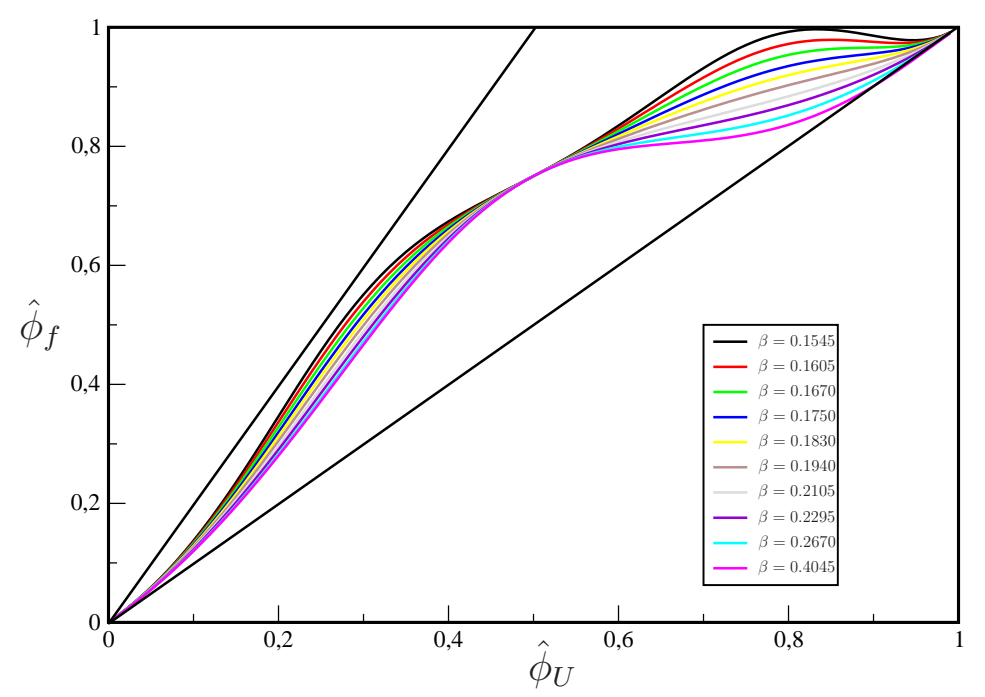

Figura 4.1: Esquema 1 em variáveis normalizadas para vários valores do parâmetro $\beta$.

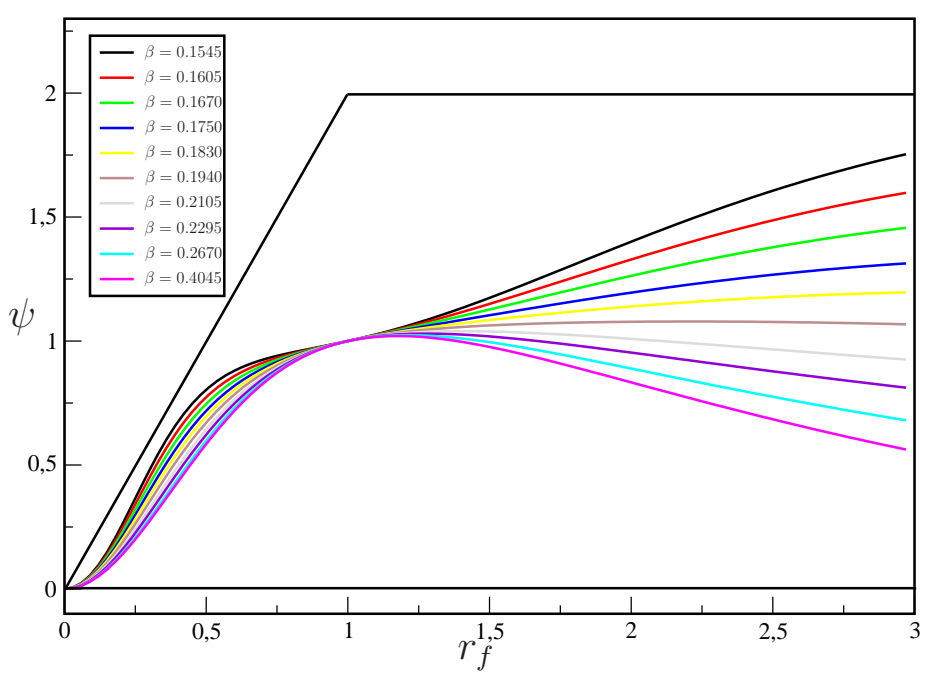

Figura 4.2: Limitador de fluxo correspondente do Esquema $1\left(r_{f} \geq 0\right)$ para vários valores do parâmetro $\beta$.

\subsection{Derivação de esquemas upwind via mínimos quadrados ponderados: os Esquemas 2, 3, 4, 5 e 6}

Nesta seção são desenvolvidos os Esquemas 2, 3, 4, 5 e 6 da família FUS-RF. Inicialmente, são desenvolvidos os limitadores de fluxo via técnica mínimos quadrados ponderados; e então os correspondentes esquemas no contexto NV são derivados. Como apresentado no capítulo 3, 
limitadores de fluxo podem ser construídos de forma otimizada, por meio da relação (3.37), isto é

$$
\psi\left(r_{f}\right)=\operatorname{Pond}\left(\frac{\left(w_{1}+\epsilon\right) \Delta \phi_{1}+\left(w_{2}+\epsilon\right) \Delta \phi_{2}}{\left(w_{1}+w_{2}+2 \epsilon\right) \Delta \phi_{1}}\right) .
$$

\subsubsection{Esquema 2}

O Esquema 2 é obtido definindo-se os pesos $w_{1}=r_{f}^{8}+a r_{f}^{3}$ e $w_{2}=b r_{f}+c$, onde os coeficientes Pond, $a, b$ e $c$ são determinados pela imposição das restrições de estabilidade (ver Deconinck [50] ou Sweby [41])

- $\psi(1)=1 \quad \Rightarrow \quad$ Pond $=1$;

- $\psi^{\prime}(1)=\frac{1}{4} \Rightarrow \operatorname{Pond}\left(\frac{(b+c)}{1+a+b+c}\right)=\frac{1}{4}$

mais as condições para simetria

- $\psi(-1)=1 \quad \Rightarrow \quad$ Pond $\left(\frac{(1-a+b-c)}{1-a-b+c}\right)=1$

- $\psi^{\prime}(-1)=-\frac{1}{4} \Rightarrow$

$$
\operatorname{Pond}\left(\frac{(-8+3 a-2 b+c)(1-a-b+c)-(1-a+b-c)(-8+3 a+b)}{(1-a-b+c)^{2}}\right)=-\frac{1}{4} \text {. }
$$

Resolvendo o sistema não linear dado pelas equações (4.15) e (4.16) via MAPLE obtém-se Pond $=1, a=-\frac{1}{7}, b=\frac{1}{7}$ e $c=\frac{1}{7}$. E em síntese, o limitador de fluxo para o Esquema 2, com a inclusão do parâmetro livre $\epsilon$ para prevenir indeterminações, é

$$
\psi\left(r_{f}\right)=\frac{r_{f}^{8}-\frac{1}{7} r_{f}^{3}+\frac{1}{7} r_{f}^{2}+\left(\frac{1}{7}+\epsilon\right) r_{f}+\epsilon}{r_{f}^{8}-\frac{1}{7} r_{f}^{3}+\frac{1}{7} r_{f}+\frac{1}{7}+2 \epsilon}, \quad \forall r_{f} .
$$

O Esquema 2 upwind em NV correspondente é obtido recorrendo-se à equação (3.15), cujo resultado é

$$
\hat{\phi}_{f}=\frac{P}{Q}, \quad \forall \hat{\phi}_{U},
$$

onde

$$
\begin{array}{r}
P=(8+28 \epsilon) \hat{\phi}_{U}^{9}-(217) \hat{\phi}_{U}^{8}+(26+728 \epsilon) \hat{\phi}_{U}^{7}-(63+1372 \epsilon) \hat{\phi}_{U}^{6}+(100+1568 \epsilon) \hat{\phi}_{U}^{5}- \\
(101+1078 \epsilon) \hat{\phi}_{U}^{4}+(62+392 \epsilon) \hat{\phi}_{U}^{3}-(21+28 \epsilon) \hat{\phi}_{U}^{2}+(3-28 \epsilon) \hat{\phi}_{U}+7 \epsilon,
\end{array}
$$


e

$$
\begin{array}{r}
Q=(16+28 \epsilon) \hat{\phi}_{U}^{9}-(12+224 \epsilon) \hat{\phi}_{U}^{7}+(34+784 \epsilon) \hat{\phi}_{U}^{6}-(62+1568 \epsilon) \hat{\phi}_{U}^{5}+(80+1960 \epsilon) \hat{\phi}_{U}^{4}- \\
(72+1568 \epsilon) \hat{\phi}_{U}^{3}+(42+1784 \epsilon) \hat{\phi}_{U}^{2}-(14+224 \epsilon) \hat{\phi}_{U}+28 \epsilon+2 .
\end{array}
$$

Usando a equação (4.18) pode-se derivar as relações

$$
\begin{gathered}
\hat{\phi}_{f}(0)=\frac{7 \epsilon}{2+28 \epsilon} \longrightarrow 0, \text { quando } \epsilon \rightarrow 0 ; \\
\hat{\phi}_{f}(1)=1, \quad \forall \epsilon ; \\
\hat{\phi}_{f}\left(\frac{1}{2}\right)=\frac{3(7 \epsilon+4)}{4(7 \epsilon+4)}, \quad \forall \epsilon ; \\
\hat{\phi}_{f}^{\prime}\left(\frac{1}{2}\right)=\frac{12+49 \epsilon+49 \epsilon^{2}}{16+56 \epsilon+49 \epsilon^{2}} \longrightarrow \frac{3}{4}, \text { quando } \epsilon \rightarrow 0 .
\end{gathered}
$$

As Figuras 4.3 e 4.4 ilustram, respectivamente, o Esquema 2 em NV e o limitador de fluxo correspondente para vários valores de $\epsilon$. Nota-se por essas figuras que os métodos (esquema e limitador) upwind $\operatorname{com} \epsilon=0.1$ e $\epsilon=0.01$ não são TVD (nas regiões em que esta é válida).

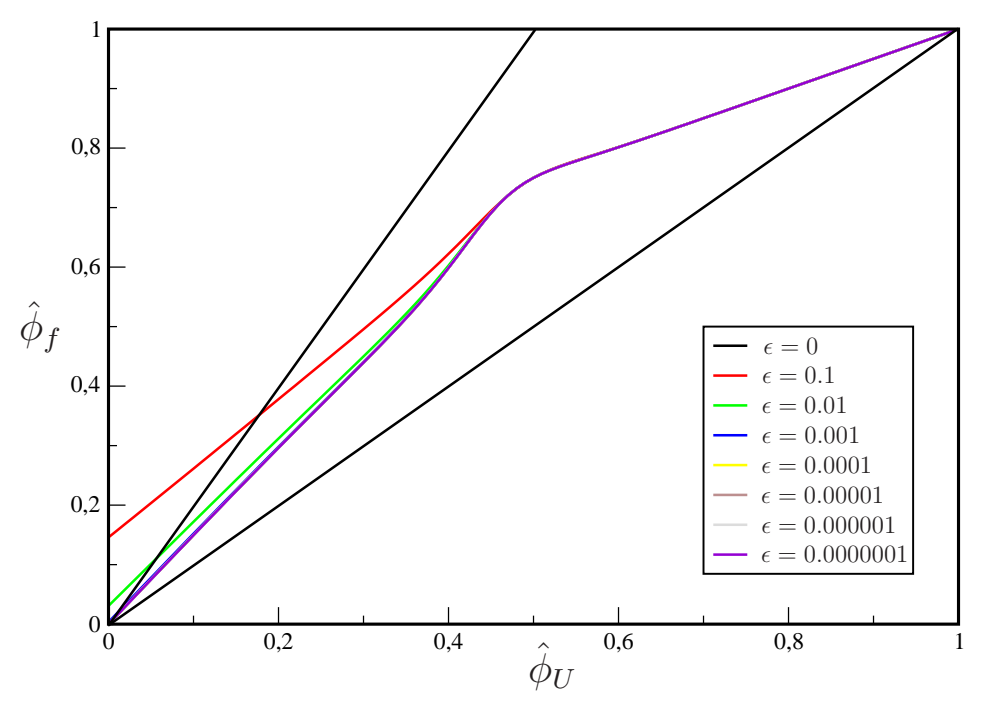

Figura 4.3: Esquema 2 em variáveis normalizadas para vários valores do parâmetro $\epsilon$.

\subsubsection{Esquema 3}

Para derivar o Esquema 3 definem-se os pesos $w_{1}=r_{f}^{4}+a r_{f}^{3}$ e $w_{2}=b$. Assim como feito para o Esquema 2, impõe-se as restrições de estabilidade 


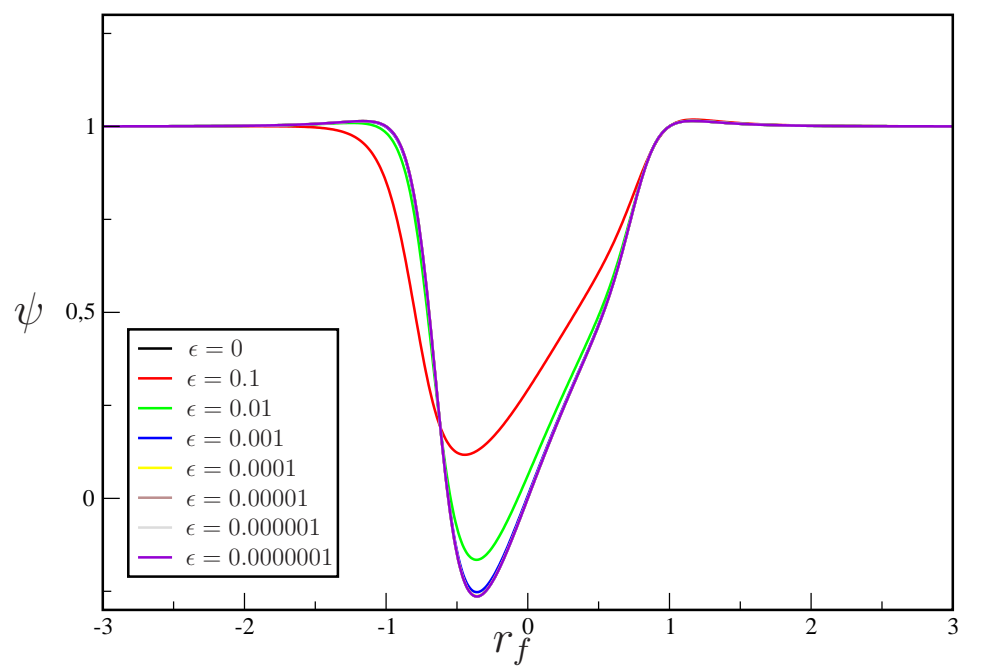

Figura 4.4: Limitador de fluxo correspondente do Esquema $2\left(\forall r_{f}\right)$ para vários valores do parâmetro $\epsilon$.

- $\psi(1)=1 \Rightarrow$ Pond $=1$;

- $\psi^{\prime}(1)=\frac{1}{4} \Rightarrow \operatorname{Pond}\left(\frac{b}{1+a+b}\right)=\frac{1}{4}$

associadas à restrição para a derivada em $r_{f}=-1$

- $\psi^{\prime}(-1)=-\frac{1}{4} \Rightarrow \operatorname{Pond}\left(\frac{(-4+3 a+b)(1-a+b)-(1-a-b)(-4+3 a)}{(1-a+b)^{2}}\right)=-\frac{1}{4}$.

Após resolver o sistema não linear obtém-se Pond $=1, a=-\frac{985}{1291}$ e $b=\frac{102}{1291}$. Assim, o limitador de fluxo do Esquema 3 é

$$
\psi\left(r_{f}\right)=\frac{1291 r_{f}^{4}-985 r_{f}^{3}+(102+1291 \epsilon) r_{f}+1291 \epsilon}{1291 r_{f}^{4}-985 r_{f}^{3}+102+2582 \epsilon}, \quad \forall r_{f} .
$$

O respectivo Esquema 3 em NV é

$$
\hat{\phi}_{f}=\frac{P}{Q}, \quad \forall \hat{\phi}_{U},
$$

onde tem-se

$$
\begin{array}{r}
P=(2582+5164 \epsilon) \hat{\phi}_{U}^{5}+(67-19365 \epsilon) \hat{\phi}_{U}^{4}+(851+25820 \epsilon) \hat{\phi}_{U}^{3}-(1224+12910 \epsilon) \hat{\phi}_{U}^{2}+ \\
(306) \hat{\phi}_{U}+1291 \epsilon,
\end{array}
$$


e

$$
\begin{aligned}
& Q=(4756+5164 \epsilon) \hat{\phi}_{U}^{4}-(2786+20656 \epsilon) \hat{\phi}_{U}^{3}+(1224+30984 \epsilon) \hat{\phi}_{U}^{2}-(816+20656 \epsilon) \hat{\phi}_{U}+ \\
& 5164 \epsilon+204 .
\end{aligned}
$$

Considerando-se a equação (4.26), pode-se aferir algumas relações importantes:

$$
\begin{gathered}
\hat{\phi}_{f}(0)=\frac{1291 \epsilon}{204+5164 \epsilon} \longrightarrow 0, \quad \text { quando } \epsilon \quad \longrightarrow \quad 0 ; \\
\hat{\phi}_{f}(1)=1, \quad \forall \epsilon ; \\
\hat{\phi}_{f}\left(\frac{1}{2}\right)=\frac{3(1291 \epsilon+204)}{4(1291 \epsilon+204)}, \quad \forall \epsilon ; \\
\hat{\phi}_{f}^{\prime}\left(\frac{1}{2}\right)=\frac{31212+460887 \epsilon+1666681 \epsilon^{2}}{41616+526728 \epsilon+1666681 \epsilon^{2}} \longrightarrow \frac{3}{4}, \text { quando } \epsilon \quad \longrightarrow \quad 0 .
\end{gathered}
$$

As Figuras 4.5 e 4.6 apresentam, respectivamente, o esquema 3 em NV e o correspondente limitador de fluxo. Nota-se que o comportamento das características NV para $\epsilon=0.1$ e $\epsilon=0.01$ mostra que para estes parâmetros o esquema não é TVD.

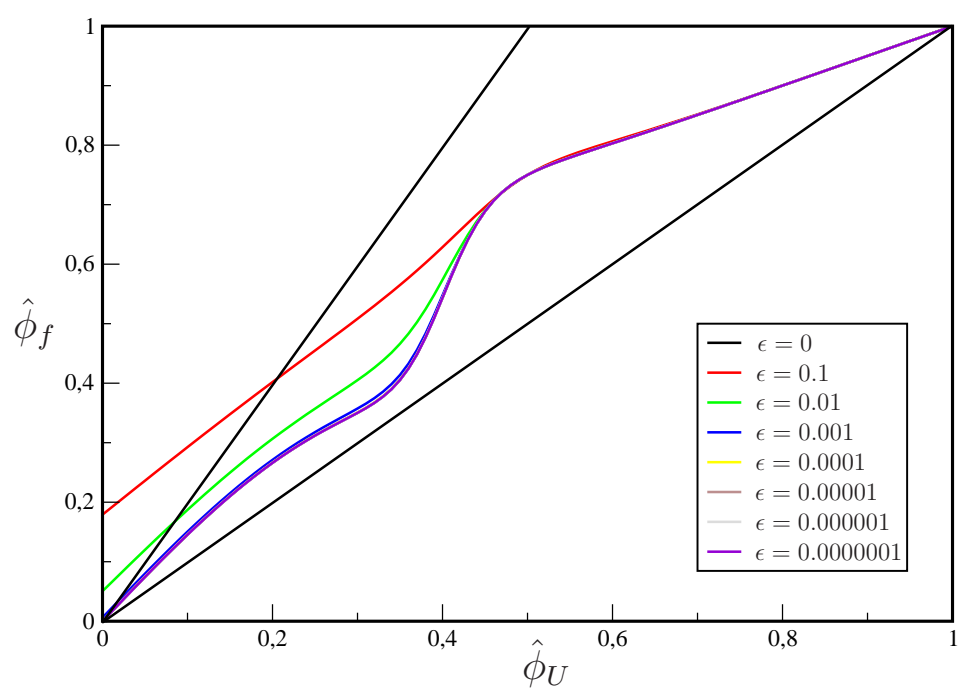

Figura 4.5: Esquema 3 em variáveis normalizadas para vários valores do parâmetro $\epsilon$.

\subsubsection{Esquema 4}

No Esquema 4 definem-se os pesos a fim de se obter um limitador racional de grau 8 então, $w_{1}=r_{f}^{8}+a r_{f}^{6}+r_{f}^{5}+b r_{f}^{3}$ e $w_{2}=r_{f}+c$. 


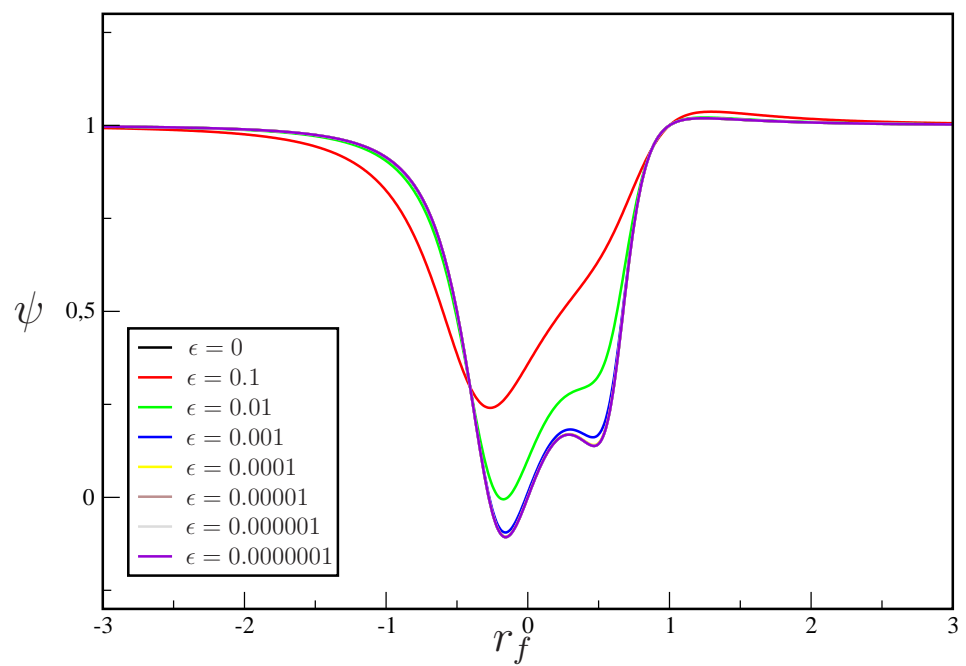

Figura 4.6: Limitador de fluxo correspondente do Esquema $3\left(\forall r_{f}\right)$ para vários valores do parâmetro $\epsilon$.

Para determinar os valores de Pond, $a, b$ e $c$ são impostas as condições de estabilidade para limitadores de fluxo, ou seja,

- $\psi(1)=1 \quad \Rightarrow \quad$ Pond $=1$;

- $\psi^{\prime}(1)=\frac{1}{4} \Rightarrow \operatorname{Pond}\left(\frac{1+c}{3+a+b+c}\right)=\frac{1}{4}$

associadas às restrições de simetria

- $\psi(-1)=1 \Rightarrow \operatorname{Pond}\left(\frac{1+a-b-c}{-1+a-b+c}\right)=1$;

- $\psi^{\prime}(-1)=-\frac{1}{4} \Rightarrow$

$$
\operatorname{Pond}\left(\frac{(-5-6 a+3 b+c)(-1+a-b+c)-(1+a-b-c)(-2-6 a+3 b)}{(-1+a-b+c)^{2}}\right)=-\frac{1}{4} \text {. }
$$

Resolvendo o sistema obtém-se Pond $=1, a=6, b=-2$ e $c=1$.

O limitador de fluxo para o Esquema 4, com a inclusão do parametro $\epsilon$ é

$$
\psi\left(r_{f}\right)=\frac{r_{f}^{8}+6 r_{f}^{6}+r_{f}^{5}-2 r_{f}^{3}+r_{f}^{2}+(1+\epsilon) r_{f}+\epsilon}{r_{f}^{8}+6 r_{f}^{6}+r_{f}^{5}-2 r_{f}^{3}+r_{f}+2 \epsilon+1}, \quad \forall r_{f}
$$

e o respectivo esquema upwind em NV é

$$
\hat{\phi}_{f}=\frac{P}{Q}, \quad \forall \hat{\phi}_{U},
$$


onde tem-se o numerador

$$
\begin{array}{r}
P=(8+4 \epsilon) \hat{\phi}_{U}^{9}-(14+31 \epsilon) \hat{\phi}_{U}^{8}+(25+104 \epsilon) \hat{\phi}_{U}^{7}-(59+196 \epsilon) \hat{\phi}_{U}^{6}+(96+224 \epsilon) \hat{\phi}_{U}^{5}- \\
(97+154 \epsilon) \hat{\phi}_{U}^{4}+(61+56 \epsilon) \hat{\phi}_{U}^{3}-(21+4 \epsilon) \hat{\phi}_{U}^{2}+(3-4 \epsilon) \hat{\phi}_{U}+\epsilon,
\end{array}
$$

e o denominador

$$
\begin{array}{r}
Q=(16+4 \epsilon) \hat{\phi}_{U}^{8}-(40+32 \epsilon) \hat{\phi}_{U}^{7}+(60+112 \epsilon) \hat{\phi}_{U}^{6}-(80+224 \epsilon) \hat{\phi}_{U}^{5}+(90+280 \epsilon) \hat{\phi}_{U}^{4}- \\
(74+224 \epsilon) \hat{\phi}_{U}^{3}+(42+112 \epsilon) \hat{\phi}_{U}^{2}-(14+32 \epsilon) \hat{\phi}_{U}+4 \epsilon+2 .
\end{array}
$$

Em vista de analisar o comportamento do Esquema 4, tem-se

$$
\begin{gathered}
\hat{\phi}_{f}(0)=\frac{\epsilon}{2+4 \epsilon} \longrightarrow 0, \quad \text { quando } \epsilon \rightarrow 0 ; \\
\hat{\phi}_{f}(1)=1, \quad \forall \epsilon ; \\
\hat{\phi}_{f}\left(\frac{1}{2}\right)=\frac{3(\epsilon+4)}{4(\epsilon+4)}, \quad \forall \epsilon ; \\
\hat{\phi}_{f}^{\prime}\left(\frac{1}{2}\right)=\frac{12+7 \epsilon+\epsilon^{2}}{16+8 \epsilon+\epsilon^{2}} \longrightarrow \frac{3}{4}, \quad \text { quando } \epsilon \rightarrow 0 .
\end{gathered}
$$

As Figuras 4.7 e 4.8 apresentam, respectivamente, o Esquema 4 em NV e o correspondente limitador de fluxo. Nota-se que apenas para $\epsilon=0.1$ as características não são TVD.

\subsubsection{Esquema 5}

O Esquema 5 é definido impondo-se os pesos $w_{1}=r_{f}^{8}+a r_{f}^{7}+b r_{f}^{6}+c r_{f}^{5}$ e $w_{2}=1 r_{f}^{3}+e r_{f}^{2}+$ $f r_{f}+1$. Os coeficientes destes pesos são determinados através das restrições

- $\psi(1)=1 \quad \Rightarrow \quad$ Pond $=1$;

- $\psi^{\prime}(1)=\frac{1}{4} \Rightarrow$ Pond $\left(\frac{e+f+2}{a+b+c+e+f+3}\right)=\frac{1}{4}$;

associadas às restrições

- $\psi(-1)=1 \Rightarrow$ Pond $\left(\frac{1-a+b-c-e+f}{1-a+b-c+e-f}\right)=1$;

- $\psi^{\prime}(-1)=-\frac{1}{4} \Rightarrow$ Pond $\left(\frac{7 a-6 b+5 c+3 e-2 f+11}{1-a+b-c+e-f}\right)-$ 


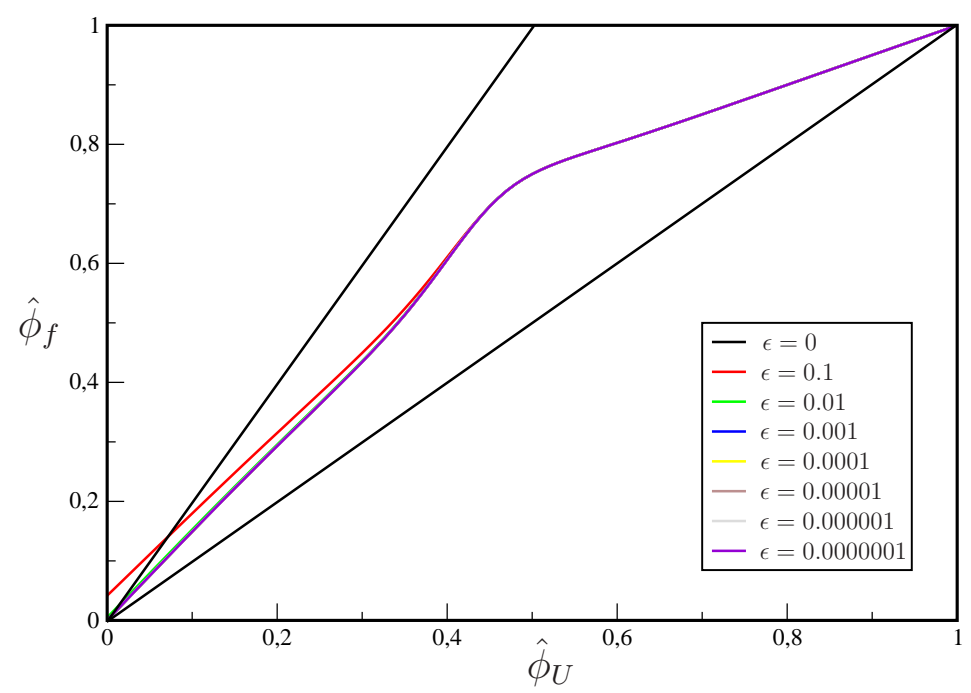

Figura 4.7: Esquema 4 em variáveis normalizadas para vários valores do parâmetro $\epsilon$.

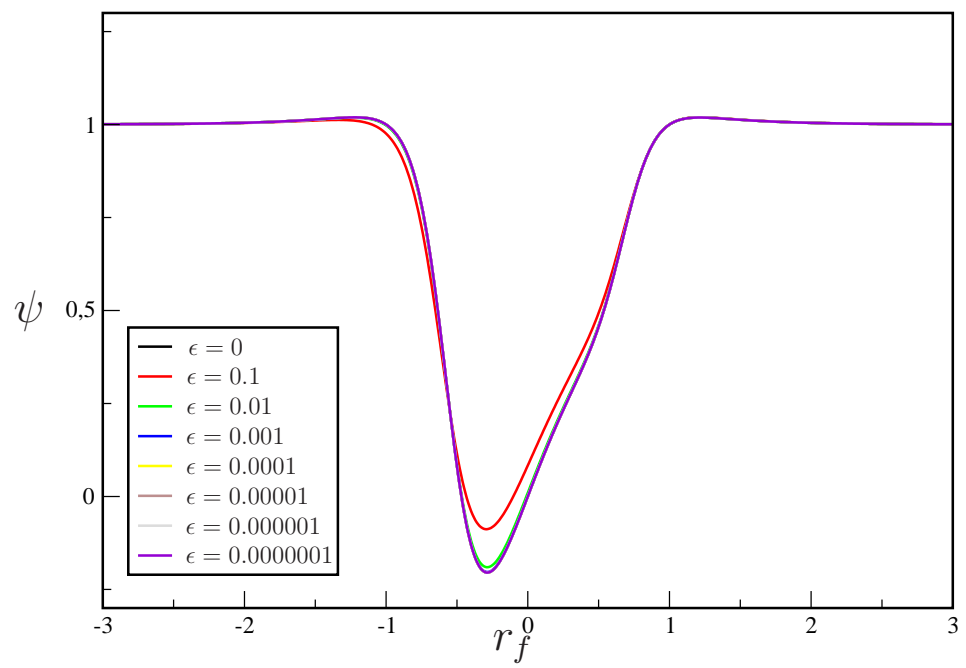

Figura 4.8: Limitador de fluxo correspondente do Esquema $4\left(\forall r_{f}\right)$ para vários valores do parâmetro $\epsilon$.

$$
\operatorname{Pond}\left(\frac{(-5+7 a-6 b+5 c-2 e+f)(1-a+b-c-e+f)}{(1-a+b-c+e-f)^{2}}\right)=-\frac{1}{4}
$$

- $\psi\left(\frac{1}{2}\right)=\frac{1}{2} \Rightarrow$ Pond $\left(\frac{\frac{1}{256}+\frac{1}{128} a+\frac{1}{64} b+\frac{1}{32} c+\frac{1}{8} e+\frac{1}{4} f+\frac{9}{16}}{\frac{1}{256}+\frac{1}{128} a+\frac{1}{64} b+\frac{1}{32} c+\frac{1}{4} e+\frac{1}{2} f++\frac{9}{8}}\right)=\frac{1}{2}$ 
- $\psi\left(-\frac{1}{2}\right)=0 \Rightarrow$ Pond $\left(\frac{\frac{1}{256}-\frac{1}{128} a+\frac{1}{64} b-\frac{1}{32} c-\frac{1}{8} e+\frac{1}{4} f+\frac{9}{16}}{\frac{1}{256}-\frac{1}{128} a+\frac{1}{64} b-\frac{1}{32} c-+\frac{1}{4} e+\frac{1}{2} f+\frac{7}{8}}\right)=0$.

Resolvendo o sistema não linear via MAPLE obtém-se Pond $=1, a=-\frac{29}{9}, b=\frac{169}{12}$, $c=-\frac{229}{36}, e=-\frac{1}{12}$ e $f=-\frac{1}{12}$.

O limitador de fluxo do Esquema 5 é

$$
\psi\left(r_{f}\right)=\frac{r_{f}^{8}-\frac{29}{9} r_{f}^{7}+\frac{169}{12} r_{f}^{6}-\frac{229}{36} r_{f}^{5}+r_{f}^{4}-\frac{1}{12} r_{f}^{3}-\frac{1}{12} r_{f}^{2}+(1+\epsilon) r+\epsilon}{r_{f}^{8}-\frac{29}{9} r_{f}^{7}+\frac{169}{12} r_{f}^{6}-\frac{229}{36} r_{f}^{5}+r_{f}^{3}-\frac{1}{12} r_{f}^{2}-\frac{1}{12} r_{f}+2 \epsilon+1}, \quad \forall \quad r_{f}
$$

Em NV, o Esquema 5 é

$$
\hat{\phi}_{f}=\frac{P}{Q}, \quad \forall \quad \hat{\phi}_{U}
$$

no qual

$$
\begin{array}{r}
P=(888+144 \epsilon) \hat{\phi}_{U}^{9}-(1262+1116 \epsilon) \hat{\phi}_{U}^{8}+(1375=3744 \epsilon) \hat{\phi}_{U}^{7}-(4138+7056 \epsilon) \hat{\phi}_{U}^{6}+ \\
(6971+8064 \epsilon) \hat{\phi}_{U}^{5}-(6075+5544 \epsilon) \hat{\phi}_{U}^{4}+(3078+2016 \epsilon) \hat{\phi}_{U}^{3}-(873+144 \epsilon) \hat{\phi}_{U}^{2}+ \\
(108-144 \epsilon) \hat{\phi}_{U}+36 \epsilon
\end{array}
$$

e o denominador

$$
\begin{array}{r}
Q=(1776+144 \epsilon) \hat{\phi}_{U}^{8}-(3856+1152 \epsilon) \hat{\phi}_{U}^{7}+(3720+4032 \epsilon) \hat{\phi}_{U}^{6}-(3860+8064 \epsilon) \hat{\phi}_{U}^{5}+ \\
(4800+10080 \epsilon) \hat{\phi}_{U}^{4}-(4050+8064 \epsilon) \hat{\phi}_{U}^{3}+(2052+4032 \epsilon) \hat{\phi}_{U}^{2}-(582+1152 \epsilon) \hat{\phi}_{U}+144 \epsilon+72 .
\end{array}
$$

Considerando-se a equação (4.42), pode-se verificar algumas relações importantes;

$$
\begin{gathered}
\hat{\phi}_{f}(0)=\frac{36 \epsilon}{72+144 \epsilon} \longrightarrow 0, \quad \text { quando } \epsilon \rightarrow 0 ; \\
\hat{\phi}_{f}(1)=1, \quad \forall \epsilon ; \\
\hat{\phi}_{f}\left(\frac{1}{2}\right)=\frac{3(33 \epsilon+9)}{4(33 \epsilon+9)}, \quad \forall \epsilon ;
\end{gathered}
$$




$$
\hat{\phi}_{f}^{\prime}\left(\frac{1}{2}\right)=\frac{3267+2079 \epsilon+324 \epsilon^{2}}{4356+2376 \epsilon+324 \epsilon^{2}} \longrightarrow \frac{3}{4}, \text { quando } \epsilon \quad \longrightarrow \quad 0 .
$$

O Esquema 5 em NV e o limitador de fluxo deste esquema estão ilustrados, respectivamente, nas Figuras 4.9 e 4.10 onde vê-se claramente que para $\epsilon=0.1$ o esquema não é TVD.

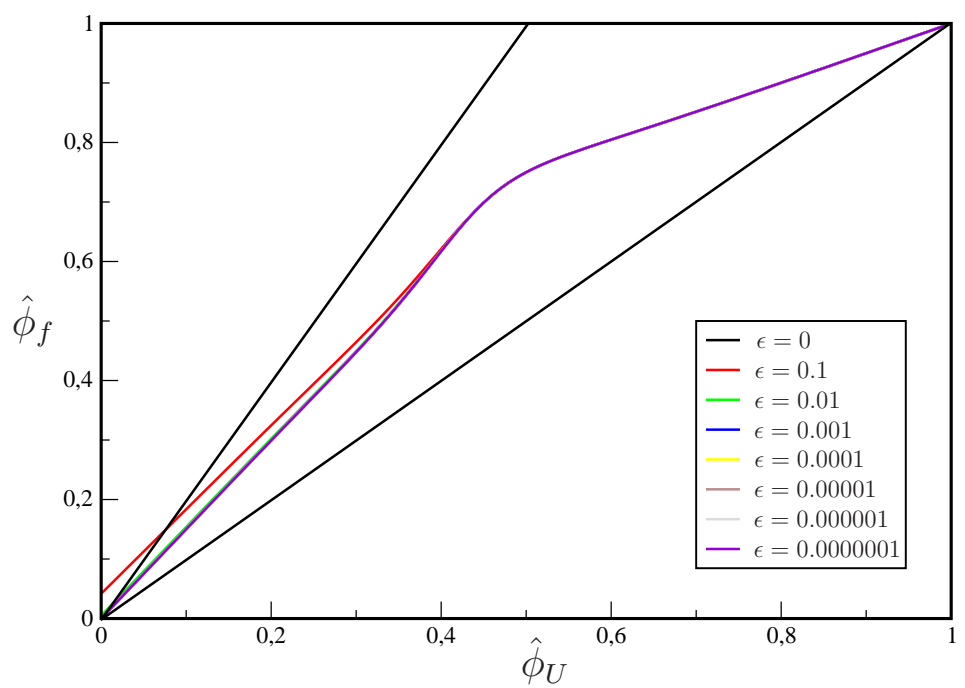

Figura 4.9: Esquema 5 em variáveis normalizadas para vários valores do parâmetro $\epsilon$.

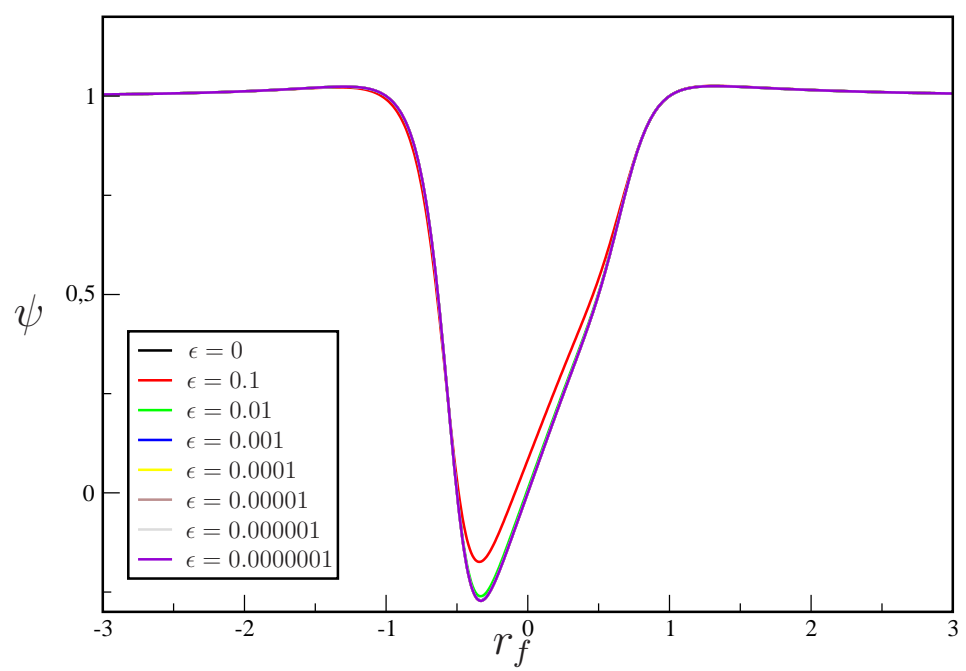

Figura 4.10: Limitador de fluxo correspondente do Esquema $5\left(\forall r_{f}\right)$ para vários valores do parâmetro $\epsilon$. 


\subsubsection{Esquema 6}

Para derivar o Esquema 6 assumem-se os pesos $w_{1}=r_{f}^{8}+r_{f}^{5}+b r_{f}^{3}$ e $w_{2}=c r_{f}+1$. Para determinar os coeficientes $b$ e $c$ recorre-se à equação (3.15), ou seja,

$$
\hat{\phi}_{f}\left(\hat{\phi}_{U}\right)=\hat{\phi}_{U}+\frac{1}{2} \psi\left(r_{f}\right)\left(1-\hat{\phi}_{U}\right)
$$

O que resulta no esquema

$\hat{\phi}_{f}\left(\hat{\phi}_{U}\right)=\phi_{U}+\frac{\text { Pond }}{2}\left(\frac{\left.\hat{\phi}_{U}^{8}\left(1-\hat{\phi}_{U}\right)+\hat{\phi}_{U}^{5}\left(1-\hat{\phi}_{U}\right)^{4}+b \hat{\phi}_{U}^{3}\left(1-\hat{\phi}_{U}\right)^{6}\right)+c \hat{\phi}_{U}^{2}\left(1-\hat{\phi}_{U}\right)^{7}+\hat{\phi}_{U}\left(1-\hat{\phi}_{U}\right)^{8}}{\left.\hat{\phi}_{U}^{8}+\hat{\phi}_{U}^{5}\left(1-\hat{\phi}_{U}\right)^{3}+b \hat{\phi}_{U}^{3}\left(1-\hat{\phi}_{U}\right)^{5}\right)+c \hat{\phi}_{U}\left(1-\hat{\phi}_{U}\right)^{7}+\left(1-\hat{\phi}_{U}\right)^{8}}\right)$.

As restrições $\hat{\phi}_{f}(0)=0$ e $\hat{\phi}_{f}(1)=1$ são satisfeitas para qualquer $b$ ou $c$, assim, considerando Pond $=1$, são impostas as restrições

$$
\begin{aligned}
& \text { - } \phi_{f}\left(\frac{1}{2}\right)=\frac{3}{4} \Rightarrow \frac{1}{2}+\frac{\text { Pond }}{2}\left(\frac{\frac{3}{512}+\frac{1}{512} b+\frac{1}{512} c}{\frac{3}{256}+\frac{1}{256} b+\frac{1}{256} c}\right)=\frac{3}{4} \\
& \text { - } \phi^{\prime}\left(\frac{1}{2}\right)=\frac{3}{4} \Rightarrow 1+\frac{\text { Pond }}{2}\left(\frac{\left(\frac{3}{256}+\frac{1}{256} b+\frac{1}{256} c\right)\left(\frac{1}{256}-\frac{3}{256} b-\frac{5}{256} c\right)}{\left(\frac{3}{256}+\frac{1}{256} b+\frac{1}{256} c\right)^{2}}\right) \\
& -\frac{\text { Pond }}{2}\left(\frac{\left(\frac{3}{512}+\frac{1}{512} b+\frac{1}{512} c\right)\left(\frac{1}{64}-\frac{1}{64} b-\frac{3}{64} c\right)}{\left(\frac{3}{256}+\frac{1}{256} b+\frac{1}{256} c\right)^{2}}\right)=\frac{3}{4} .
\end{aligned}
$$

Resolvendo o sistema não linear obtém-se $b=-\frac{361}{273}$ e $c=-\frac{634}{819}$. Assim, o Esquema $6 \mathrm{em}$ NV é

$$
\hat{\phi}_{f}=\frac{P}{Q}, \quad \forall \quad \hat{\phi}_{U}
$$

onde

$$
\begin{array}{r}
P=(5442+3276 \epsilon) \hat{\phi}_{U}^{9}-(34845+25389 \epsilon) \hat{\phi}_{U}^{8}+(114153+85176 \epsilon) \hat{\phi}_{U}^{7}-(205800+160524 \epsilon) \hat{\phi}_{U}^{6}+ \\
(233964+183456 \epsilon) \hat{\phi}_{U}^{5}-(173202+126126 \epsilon) \hat{\phi}_{U}^{4}+(81017+45864 \epsilon) \hat{\phi}_{U}^{3}+ \\
(21558-3276 \epsilon) \hat{\phi}_{U}^{2}+(2457-3276 \epsilon) \hat{\phi}_{U}+819 \epsilon,
\end{array}
$$


$\mathrm{e}$

$$
\begin{array}{r}
Q=(5072+3276 \epsilon) \hat{\phi}_{U}^{8}-(27896+26208 \epsilon) \hat{\phi}_{U}^{7}+(89238+91728 \epsilon) \hat{\phi}_{U}^{6}-(156130+183456 \epsilon) \hat{\phi}_{U}^{5}+ \\
(169870+229320 \epsilon) \hat{\phi}_{U}^{4}-(120522+183456 \epsilon) \hat{\phi}_{U}^{3}+(54740+91728 \epsilon) \hat{\phi}_{U}^{2}- \\
(14372+26208 \epsilon) \hat{\phi}_{U}+3276 \epsilon+1638 .
\end{array}
$$

O respectivo limitador de fluxo é

$$
\psi\left(r_{f}\right)=\frac{r_{f}^{8}+r_{f}^{5}-\frac{361}{273} r_{f}^{3}-\frac{634}{819} r_{f}^{2}+(1+\epsilon) r_{f}+\epsilon}{r_{f}^{8}+r_{f}^{5}-\frac{361}{273} r_{f}^{3}-\frac{634}{819} r_{f}+2 \epsilon+1}, \quad \forall r_{f} .
$$

Algumas relações são observadas quando se impõe o parâmetro $\epsilon$ para prevenir indeterminações, ou seja,

$$
\begin{gathered}
\hat{\phi}_{f}(0)=\frac{819 \epsilon}{1638+3676 \epsilon} \longrightarrow 0, \quad \text { quando } \epsilon \quad \longrightarrow \quad 0 ; \\
\hat{\phi}_{f}(1)=1, \quad \forall \epsilon ; \\
\hat{\phi}_{f}\left(\frac{1}{2}\right)=\frac{3(819 \epsilon+370)}{4(819 \epsilon+370)}, \quad \forall \epsilon ; \\
\hat{\phi}_{f}^{\prime}\left(\frac{1}{2}\right)=\frac{410700+2121210 \epsilon+2683044 \epsilon^{2}}{547600+2424240 \epsilon+2683044 \epsilon^{2}} \longrightarrow \frac{3}{4}, \text { quando } \quad \longrightarrow \quad \longrightarrow \quad 0 .
\end{gathered}
$$

A Figura 4.11 ilustra o comportamento do esquema upwind 6 e a Figura 4.12 apresenta o comportamento do limitador de fluxo correspondente. Ambas aferem que para $\epsilon$ pequeno $(\epsilon \longrightarrow 0)$ satisfaz-se a propriedade TVD. 


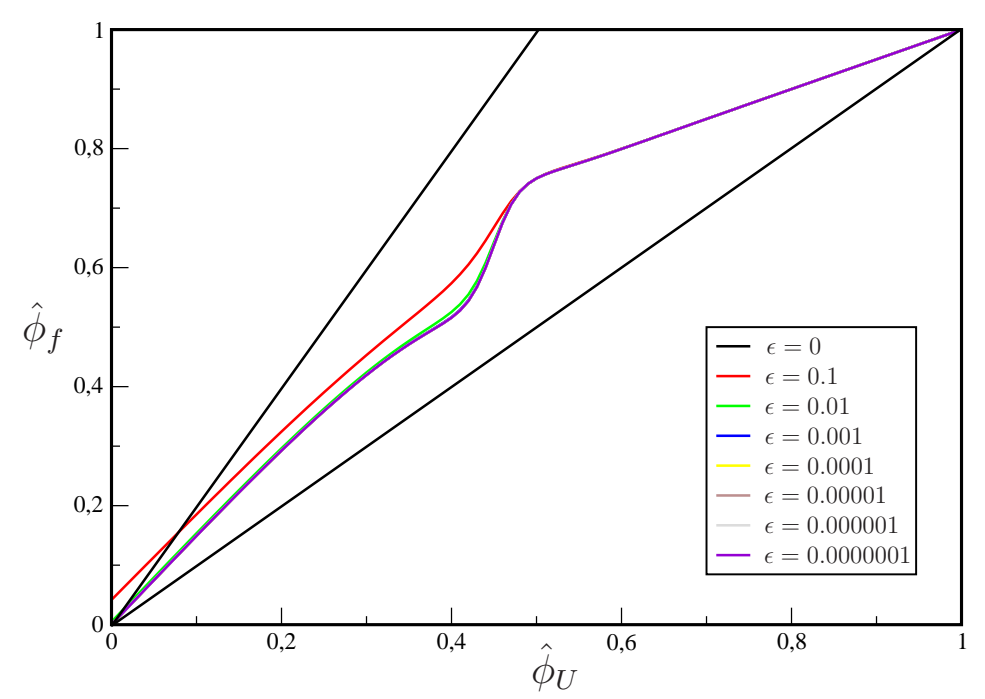

Figura 4.11: Esquema 6 em variáveis normalizadas para vários valores do parâmetro $\epsilon$.

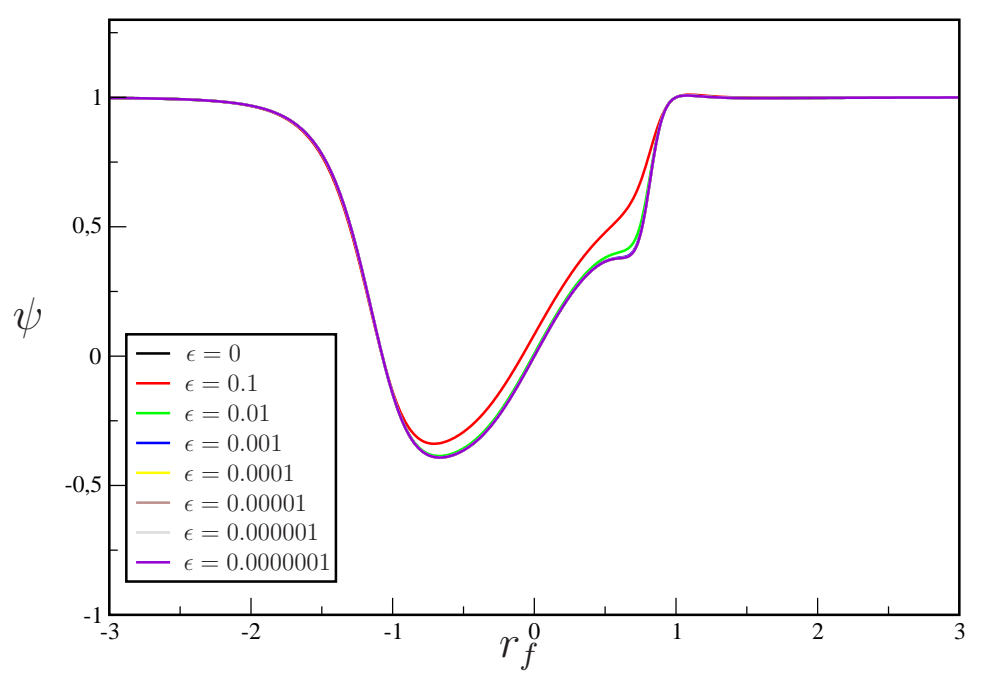

Figura 4.12: Limitador de fluxo correspondente do Esquema $6\left(\forall r_{f}\right)$ para vários valores do parâmetro $\epsilon$. 
CAPÍTUlO

\section{Modelagem computacional}

Neste capítulo apresentam-se a discretização das equações descritas no capítulo 2 e os métodos computacionais para resolvê-las. Discute-se também como (e onde) são implementados os limitadores de fluxo e os esquemas em NV associados.

\subsection{Equações hiperbólicas}

As equações de advecção de escalares, de Burgers, de águas rasas e de Euler foram resolvidas numericamente utilizando-se o ambiente de simulação CLAWPACK (Conservation LAW PACKage) de LeVeque [21], que implementa leis de conservação no contexto da metodologia padrão de volumes finitos. Neste software, as equações são consideradas na forma quase-linear, como em (2.1), cuja transformação para esta forma estão apresentadas no Apêndice A. Por simplicidade, e sem perda de generalidade, considera-se o caso 1D do sistema geral (2.1)

$$
\frac{\partial \phi}{\partial t}+A \frac{\partial \phi}{\partial x}=\zeta
$$

onde $A=A(\phi, t)$ é a matriz jacobiana associada. A reconstrução para a variável $\phi$ (ver [21]) implementada no código CLAWPACK é dada por

$$
\phi_{(i, j+1)}=\phi_{(i, j)}-\frac{\delta_{t}}{\delta_{x}}\left(A^{-} \Delta \phi_{1}+A^{+} \Delta \phi_{2}\right)-\frac{\delta_{t}}{\delta_{x}}\left(\widetilde{F}_{i+\frac{1}{2}}-\widetilde{F}_{i-\frac{1}{2}}\right)
$$

onde $\widetilde{F}_{i+\frac{1}{2}}$ e $\widetilde{F}_{i-\frac{1}{2}}$ são termos de correção de alta resolução. As flutuações (à direita) $A^{-} \Delta \phi_{1}$ e (à esquerda) $A^{+} \Delta \phi_{2}$ são definidas como

$$
A^{-} \Delta \phi_{1}=\sum_{q=1}^{m}\left(s_{i+\frac{1}{2}}^{q}\right)^{-} W_{i-\frac{1}{2}}^{q}
$$


$\mathrm{e}$

$$
A^{+} \Delta \phi_{2}=\sum_{q=1}^{m}\left(s_{i-\frac{1}{2}}^{q}\right)^{+} W_{i-\frac{1}{2}}^{q},
$$

nas quais $m$ é o número de onda; $W_{[]}^{q}$ é uma espécie de salto da $q$-ésima onda com velocidade $s^{q} ;(s)^{-}=\min (s, 0)$ e $(s)^{+}=\max (s, 0)$. No CLAWPACK, resolvedores de Riemann calculam inicialmente as ondas e as velocidades, e depois, com estes dados, calculam as flutuações. Finalmente, para avaliar a reconstrução (5.2), calculam-se os corretores de fluxo, onde, por exemplo,

$$
\widetilde{F}_{i-\frac{1}{2}}=\frac{1}{2} \sum_{q=1}^{m}\left|s_{1-\frac{1}{2}}^{q}\right|\left(1-\frac{\delta t}{\delta x}\left|s_{1-\frac{1}{2}}^{q}\right|\right) \widetilde{W}_{i-\frac{1}{2}}^{q},
$$

em que $\widetilde{W}_{i-\frac{1}{2}}^{q}$ é uma versão limitada de $W_{i-\frac{1}{2}}^{q}$ (ver [21]) obtida como

$$
\widetilde{W}_{i-\frac{1}{2}}^{q}=\widetilde{\alpha}_{i-\frac{1}{2}} \theta^{q}
$$

onde $\theta^{q}$ é o $q$-ésimo autovetor de $A$ e $\widetilde{\alpha}_{i-\frac{1}{2}}$ é um coeficiente dado por

$$
\widetilde{\alpha}_{i-\frac{1}{2}}=\Theta^{-1}\left[\phi_{(i, j)}-\phi_{(i-1, j)}\right]^{T} \psi\left(r_{i-\frac{1}{2}}^{q}\right) .
$$

Na equação (5.7), $\Theta$ é a matriz de autovetores de $A$; e $\psi\left(r_{i-\frac{1}{2}}^{q}\right)$ é um limitador de fluxo, com o sensor de suavidade $r_{i-\frac{1}{2}}^{q}$ dado por

$$
r_{i-\frac{1}{2}}^{q}=\frac{\alpha_{I-\frac{1}{2}}}{\alpha_{i-\frac{1}{2}}}, \quad I=\left\{\begin{array}{cc}
i-1, & s_{i-\frac{1}{2}}^{q}<0, \\
i+1, & s_{i+\frac{1}{2}}^{q}>0 .
\end{array}\right.
$$

O cálculo para $\widetilde{F}_{i+\frac{1}{2}}$ segue procedimento similar.

Neste trabalho, os limitadores de fluxo discutidos anteriormente (ver capítulos 3 e 4) foram implementados no código CLAWPACK via equação (5.7). O método numérico para resolver as equações de conservação hiperbólicas é explícito, e sua estabilidade é governada pela condição CFL (ver [2]). A equação de reconstrução (5.2) pode ser estendida facilmente aos casos multidimensionais (ver detalhes em [21]).

\subsection{Equações de Navier-Stokes}

As equações de conservação para escoamentos incompressíveis (Navier-Stokes - equações ((2.10) e (2.11) ) foram resolvidas com o código Freeflow de Castelo et al. [4] (ver também [30]). Neste código, está implementada a metodologia GENSMAC (Generalized simplied marker and cell) de Tomé [44], uma variante do método de projeção de Chorin [5] e desenvolvido por Harlow e Welch, que baseia-se na ideia de representação do fluido por partículas marcadoras, as quais são passivamente transportadas pelo fluido. Nesta metodologia, utiliza-se uma malha 
deslocada (staggered grid), na qual as variáveis escalares (como a pressão, por exemplo) são avaliadas no centro das células computacionais e os campos vetoriais (como a velocidade) são definidas nas faces destas células, conforme ilustra a Figura 5.1 para o caso 3D.

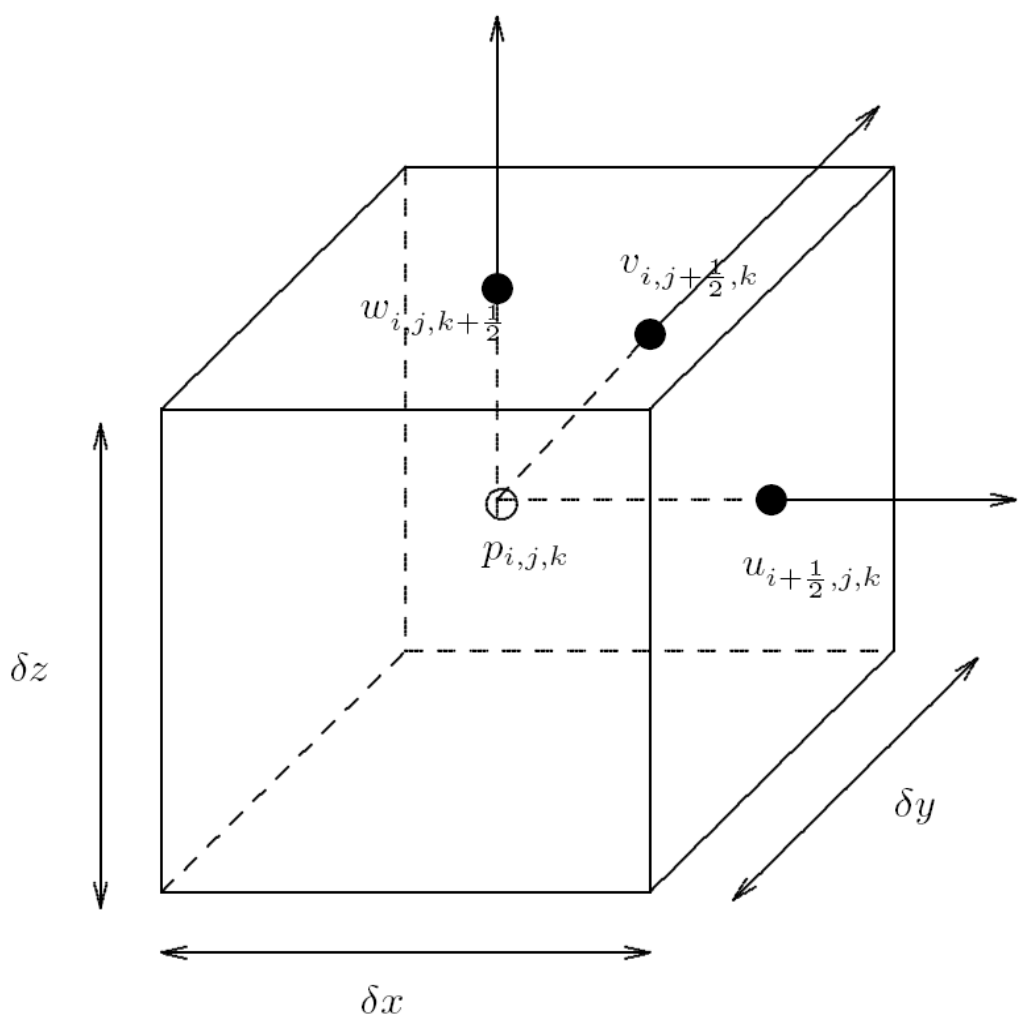

Figura 5.1: Exemplo de uma célula computacional 3D, mostrando onde as variáveis são avaliadas.

Para os termos convectivos das equações completas de Navier-Stokes (2.10) e (2.11) (os procedimentos são similares para os casos 2D e com simetria radial), a aplicação dos esquemas convectivos em NV é feita como segue. Para a malha deslocada usada neste trabalho, a face $f$ para a discretização pode ser uma das seguintes faces do volume de controle ilustrado na Figura 5.1:

$$
\left(i+\frac{1}{2}, j, k\right), \quad\left(i, j+\frac{1}{2}, k\right) \quad \text { ou } \quad\left(i, j, k+\frac{1}{2}\right) .
$$

A variável convectada $\phi$ calculada por um dos esquema upwind vistos anteriormente pode ser uma das componentes $u, v$ ou $w$. Por simplicidade, será apresentada somente a discretização do termo de advecção não linear da componente $u$ (conservação do momento na direção $x$ ) das equações (2.11). A discretização dos outros termos convectivos segue ideias semelhantes. $\mathrm{Na}$ posição $\left(i+\frac{1}{2}, j, k\right)$ da malha 3D (ver Figura 5.1), esse termo advectivo é aproximado pelo esquema numérico 


$$
\begin{aligned}
\left.\left(\frac{\partial(u u)}{\partial x}+\frac{\partial(u v)}{\partial y}+\frac{\partial(u w)}{\partial z}\right)\right|_{i+\frac{1}{2}, j, k} & \approx \frac{\bar{u}_{i+1, j, k} \cdot u_{i+1, j, k}-\bar{u}_{i, j, k} \cdot u_{i, j, k}}{\delta_{x}} \\
+ & \frac{\bar{v}_{i+\frac{1}{2}, j+\frac{1}{2}, k} \cdot u_{i+\frac{1}{2}, j+\frac{1}{2}, k}-\bar{v}_{i+\frac{1}{2}, j-\frac{1}{2}, k} \cdot u_{i+\frac{1}{2}, j-\frac{1}{2}, k}}{\delta_{y}} \\
+ & \frac{\bar{w}_{i+\frac{1}{2}, j, k+\frac{1}{2}} \cdot u_{i+\frac{1}{2}, j, k+\frac{1}{2}}-\bar{w}_{i+\frac{1}{2}, j, k-\frac{1}{2}} \cdot u_{i+\frac{1}{2}, j, k-\frac{1}{2}}}{\delta_{z}},
\end{aligned}
$$

onde $\bar{u}_{i+1, j, k}, \bar{u}_{i, j, k}, \bar{v}_{i+\frac{1}{2}, j+\frac{1}{2}, k}, \bar{v}_{i+\frac{1}{2}, j-\frac{1}{2}, k}, \bar{w}_{i+\frac{1}{2}, j, k+\frac{1}{2}}$ e $\bar{w}_{i+\frac{1}{2}, j, k-\frac{1}{2}}$ são os fluxos de massa os quais são obtidos usando-se médias simples. As velocidades convectadas na equação (5.9) (tais como $u_{i+1, j, k}, u_{i, j, k}, u_{i+\frac{1}{2}, j+\frac{1}{2}, k}$, etc) são calculadas usando-se um esquema upwind em NV, como por exemplo os da classe FUS-RF. Para exemplificação, apresenta-se a seguir o cálculo da variável convectada $u$ no ponto $(i+1, j, k)$, isto é $u_{i+1, j, k}$.

Inicia-se o processo determinando o sinal da velocidade de convecção $\bar{u}_{i+1, j, k}$. Com essa informação do fluxo, definem-se, automaticamente, as posições à jusante $D$, à montante $U$ e à mais a montante $R$. As aproximações para $u_{i+1, j, k}$, usando por exemplo o Esquema 1, são obtidas então como segue (as aproximações usando-se os Esquemas 2, 3, 4, 5 e 6 seguem ideias similares):

- Quando $\bar{u}_{i+1, j, k}>0$ tem-se $\hat{u}_{i+\frac{1}{2}, j, k}=\frac{u_{i+\frac{1}{2}, j, k}-u_{i-\frac{1}{2}, j, k}}{u_{i+\frac{3}{2}, j, k}-u_{i-\frac{1}{2}, j, k}}$, e assim

$$
u_{i+1, j, k}=\left\{\begin{array}{lll}
u_{R}+\left(u_{D}-u_{R}\right) \hat{\phi}_{f}\left(\hat{u}_{U}\right), & \text { se } & \hat{u}_{U} \in[0,1], \\
u_{U}, & \text { se } & \hat{u}_{U} \notin[0,1]
\end{array}\right.
$$

onde

$$
D=\left(i+\frac{3}{2}, j, k\right), \quad U=\left(i+\frac{1}{2}, j, k\right), \quad R=\left(i-\frac{1}{2}, j, k\right) .
$$

- Quando $\bar{u}_{i+1, j, k}<0$ tem-se $\hat{u}_{i+\frac{3}{2}, j, k}=\frac{u_{i+\frac{3}{2}, j, k}-u_{i+\frac{5}{2}, j, k}}{u_{i+\frac{1}{2}, j, k}-u_{i+\frac{5}{2}, j, k}}$ e assim

$$
u_{i+1, j, k}=\left\{\begin{array}{lll}
u_{R}+\left(u_{D}-u_{R}\right) \hat{\phi}_{f}\left(\hat{u}_{U}\right), & \text { se } & \hat{u}_{U} \in[0,1], \\
u_{U}, & \text { se } & \hat{u}_{U} \notin[0,1]
\end{array}\right.
$$

na qual

$$
D=\left(i+\frac{1}{2}, j, k\right), \quad U=\left(i+\frac{3}{2}, j, k\right), \quad R=\left(i+\frac{5}{2}, j, k\right) .
$$

Aproximações para todos os termos da equação de Navier-Stokes estão descritos no Apêndice B. 
O seguinte critério foi utilizado para selecionar o tamanho de passo no tempo (os casos 2D e com simetria radial são similares)

$$
\delta_{t}=\min \left\{F A C T_{1} \cdot \delta_{t \mathrm{CFL}}, F A C T_{2} \cdot \delta_{t \mathrm{VISC}}\right\},
$$

onde $F A C T_{1}$ e $F A C T_{2}$ são constantes escolhidas para assegurar que os cálculos são estáveis com

$$
\delta_{t \mathrm{CFL}}=\max \left\{\frac{\delta_{x}}{|u|}, \frac{\delta_{y}}{|v|}, \frac{\delta_{z}}{|w|}\right\} \quad \text { e } \quad \delta_{\mathrm{tVISC}}=\frac{\mathrm{R}_{\mathrm{e}}}{2} \frac{\delta_{\mathrm{x}}^{2} \delta_{\mathrm{y}}^{2} \delta_{\mathrm{z}}^{2}}{\delta_{\mathrm{x}}^{2} \delta_{\mathrm{y}}^{2}+\delta_{\mathrm{x}}^{2} \delta_{\mathrm{z}}^{2}+\delta_{\mathrm{y}}^{2} \delta_{\mathrm{z}}^{2}} .
$$

Com base na metodologia GENSMAC [44], as equações (2.10) e (2.11) são resolvidas como segue. Supõe-se que no tempo $t=t_{0}$ se conhece a configuração do escoamento do fluido, isto é, sabe-se as posições das partículas marcadoras representando o fluido no domínio de solução. Admite-se também que, nesse mesmo instante de tempo, $\tilde{p}$, uma pseudo-pressão arbitrária satisfazendo a condição sobre superfícies livres, é conhecida. Este campo de pressão é construído empregando-se as condições corretas na superfície livre e é escolhido de forma arbitrária (mas fixada, por exemplo $\tilde{p}=0$ ) no interior do domínio. Considera-se ainda conhecido $\tilde{\mathbf{u}}$, um campo de velocidades aproximado não necessariamente satisfazendo a restrição (2.10). Define-se a partir das equações de Navier-Stokes

$$
\frac{\partial \tilde{\mathbf{u}}}{\partial t}=-\nabla \cdot(\mathbf{u u})-\nabla \tilde{p}+\frac{1}{R_{e}} \nabla^{2} \mathbf{u}+\frac{1}{F_{r}^{2}} \mathbf{g} .
$$

Desde que no tempo $t=t_{0}, \mathbf{u}(\mathbf{x}, t)$ e $p(\mathbf{x}, t)$ também satisfazem essas equações, tem-se, nesse tempo, após subtrair (5.10) de (2.11), que

$$
\frac{\partial}{\partial t}(\mathbf{u}-\tilde{\mathbf{u}})=-\nabla(p-\tilde{p})
$$

A aplicação do rotacional em ambos os lados de (5.11) leva a

$$
\frac{\partial}{\partial t}(\nabla \times(\mathbf{u}-\tilde{\mathbf{u}}))=0
$$

Aproximando a derivada anterior por diferenças avançadas e lembrando que em $t=t_{0}, \mathbf{u}=\tilde{\mathbf{u}}$, tem-se num tempo posterior $t$

$$
\nabla \times(\mathbf{u}-\tilde{\mathbf{u}}) \approx 0
$$

O significado físico de (5.13) é que as vorticidades associadas a u e $\tilde{\mathbf{u}}$, no tempo t, são muito próximas. Ainda mais, o escoamento com respeito ao campo $(\mathbf{u}-\tilde{\mathbf{u}})$ é praticamente irrotacional. Isso leva a admitir a existência de uma função escalar $\Upsilon$, chamada potencial de velocidades de 
maneira que no tempo $t$

$$
\mathbf{u}-\tilde{\mathbf{u}}=-\nabla \Upsilon
$$

Aplicando a divergência em ambos os lados de (5.14) e impondo conservação de massa, obtém-se a equação de Poisson no tempo t para o potencial auxiliar $\Upsilon$

$$
\nabla^{2} \Upsilon(\mathbf{x}, t)=\nabla \cdot \tilde{\mathbf{u}}(\mathbf{x}, t)
$$

Acontece que no tempo atual $t$ não se conhece a configuração do fluido no domínio de solução. Uma alternativa é resolver a equação de Poisson no tempo $t=t_{0}$, argumentando que o tamanho de passo $\delta t$ é muito pequemo, e portanto as configurações do fluido em $t_{0}$ e $t$ diferem muito pouco.

Para a simulação de escoamentos de fluidos, admite-se que, num dado instante de tempo, por exemplo $t_{0}$, as variáveis dependentes são conhecidas e as condições de fronteira associadas estão completamente especificadas. As equações de Navier-Stokes são resolvidas de uma maneira explícita (método de Euler para a marcha no tempo). Mais precisamente, um ciclo computacional consiste em atualizar as variáveis discretas, a partir do tempo inicial $t_{0}$, no tempo $\mathrm{t}=t_{0}+\delta t$, utilizando-se os passos descritos a seguir:

- PASSO 1: No tempo inicial, ou num ciclo prévio, computar um campo de velocidade tentativo $\tilde{\mathbf{u}}(\mathbf{x}, t)$ em $t_{0}+\delta t$, com as mesmas condições iniciais e de contorno de $\mathbf{u}$, utilizando-se $(5.10)$;

- PASSO 2: Resolver a equação de Poisson para o potencial auxiliar $\Upsilon$, a partir de (5.15), com as seguintes condições de fronteira: em contornos rígidos Neumann homogênea; e em fronteiras livres Dirichlet homogênea. No caso dos contornos rígidos, toma-se a condição $\nabla \Upsilon=0$ de modo a satisfazer a condição (5.14), fornecendo assim os valores corretos para u nestas fronteiras;

- PASSO 3: Corrigir o campo de velocidade a partir de (5.14);

- PASSO 4: Atualizar a pressão da seguinte forma: aproximando a derivada temporal em (5.11) por diferença progressiva, lembrando que em $t=t_{0}, \tilde{\mathbf{u}}=\mathbf{u}$ e utilizando (5.14), obtem-se

$$
p\left(x_{i}, t\right)=\tilde{p}\left(x_{i}, t\right)+\frac{\Upsilon\left(x_{i}, t\right)}{\delta t}
$$

- PASSO 5: Determinar, no caso de problemas com superfícies livres, as novas posições das partículas marcadoras virtuais pela representação lagrangeana do movimento $\left(\frac{d \mathbf{x}}{d t}=\mathbf{u}\right)$; 
- PASSO 6: Atualizar as condições de fronteira necessárias para o próximo ciclo e retornar ao PASSO 1. 


\section{Resultados numéricos para leis de conservação hiperbólicas}

Neste capítulo, investiga-se o comportamento dos esquemas upwind da classe FUS-RF em sistemas de leis de conservação hiperbólicas. Em particular, são simulados alguns problemas modelados pela equação linear de advecção e pelas equações não lineares de Burgers, Euler e águas rasas; nos casos 1D e 2D. É feita uma análise da variação do parâmetro livre $\beta$ no Esquema 1 e da perturbação $\epsilon$ nos Esquemas 2, 3, 4, 5 e 6 . As soluções numéricas obtidas são comparadas com às derivadas dos esquemas convencional van Albada [46] e atual EPUS [7]. São estimadas também para as várias EDPs a TV, a ordem de convergência observada e a constante do erro em regime assintótico.

\subsection{Resultados para advecção de escalares}

Resultados para advecção de escalares modelada por (2.3) com condições iniciais suaves e descontínuas são apresentados. Nos testes apresentados a seguir, a velocidade advectiva $u$ é definida como sendo constante e igual a 1. O termo convectivo da equação é aproximado pelos esquemas van Albada, EPUS, e pela família de esquemas upwind FUS-RF.

Teste 1: Neste teste é utilizada a condição inicial suave

$$
\phi_{0}(x)=\exp \left(-100(x-0.4)^{2}\right) / 10, \quad x \in[-2,2] .
$$

Para a resolução desse problema, é considerada uma malha de 300 células computacionais, um tempo final de simulação $T=0.2$ e número de Courant $C F L=0.5$. Na Figura 6.1 são apresentados a solução exata e os resultados obtidos com os esquemas FUS-RF, sendo 
o Esquema 1 utilizado com os valores $\beta=0.1545, \beta=0.1605, \beta=0.1670, \beta=0.1750$, $\beta=0.1830, \beta=0.1940, \beta=0.2105, \beta=0.2295, \beta=0.2670$ e $\beta=0.4045$; e os demais esquemas (Esquemas 2, 3, 4, 5 e 6) utilizados com os valores da perturbação no intervalo $\epsilon=0$ à $\epsilon=10^{-5}$.

(a) Esquema 1

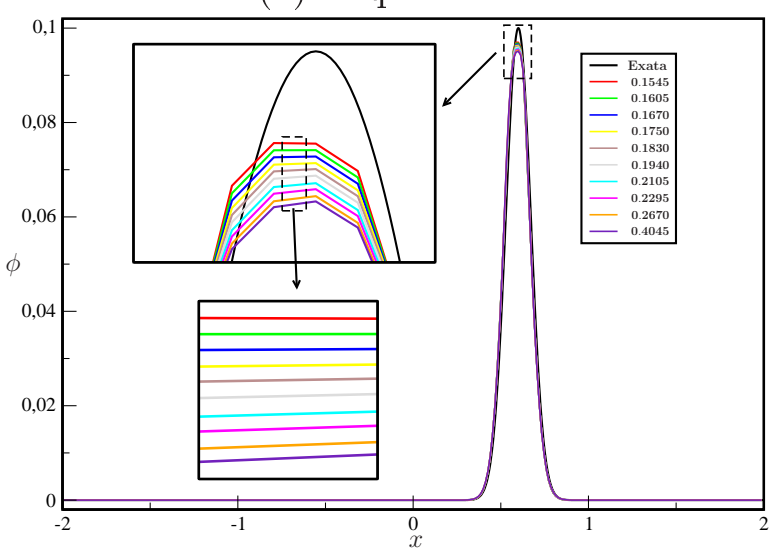

(c) Esquema 3

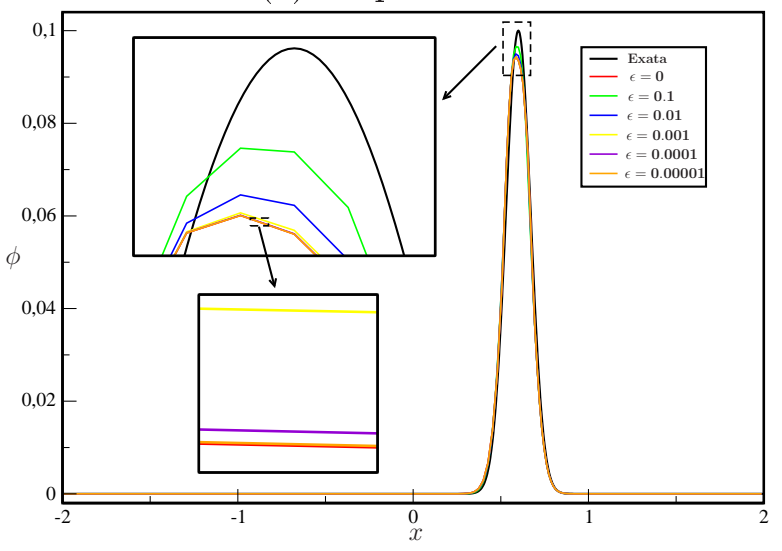

(e) Esquema 5

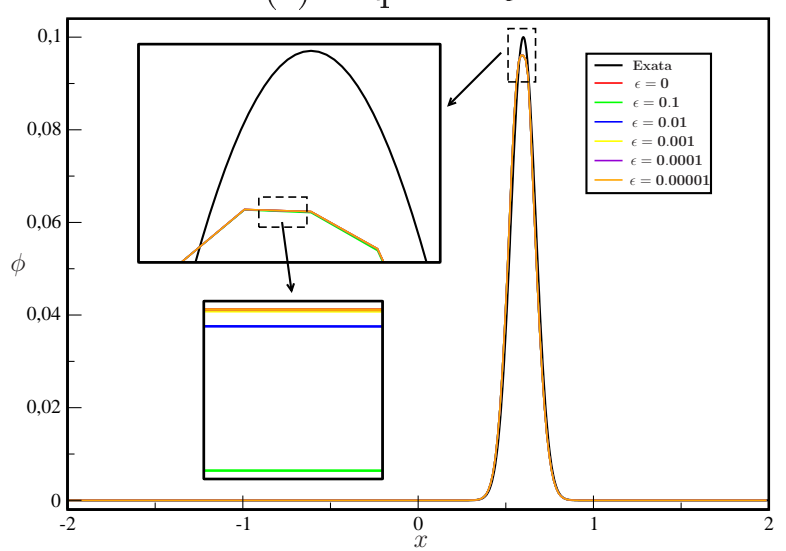

(b) Esquema 2

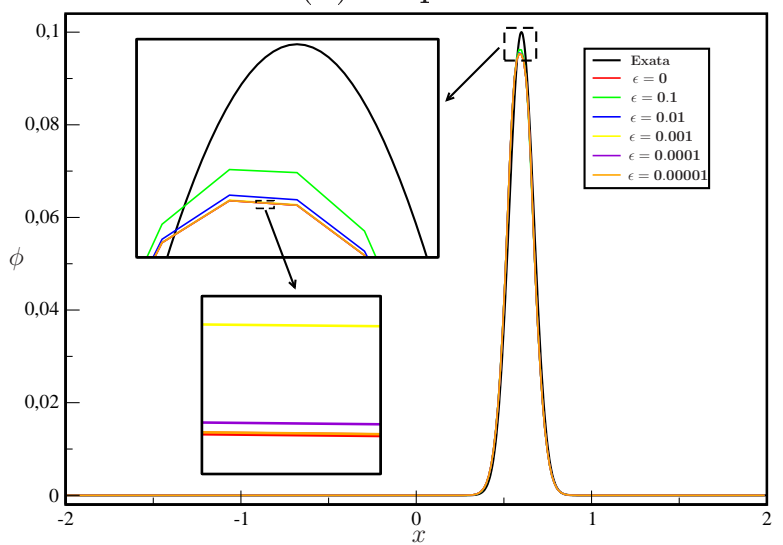

(d) Esquema 4

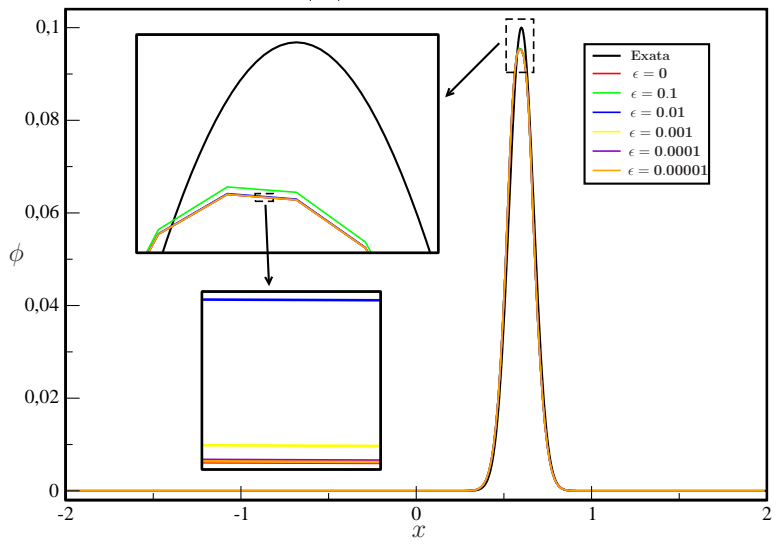

(f) Esquema 6

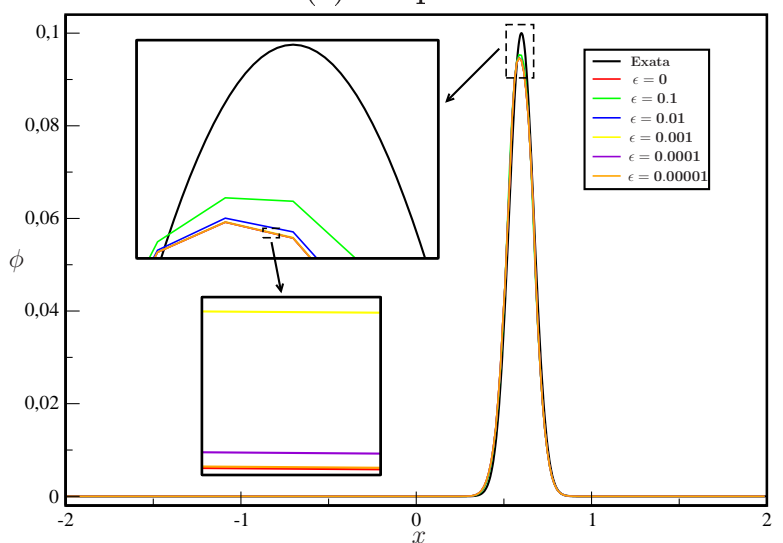

Figura 6.1: Solução exata e resultados obtidos com os esquemas FUS-RF para advecção de um escalar. (a) Esquema 1 utilizando vários valores do parâmetro $\beta$; (b)-(f) Esquemas $2-6$ utilizando vários valores do parâmetro $\epsilon$. 


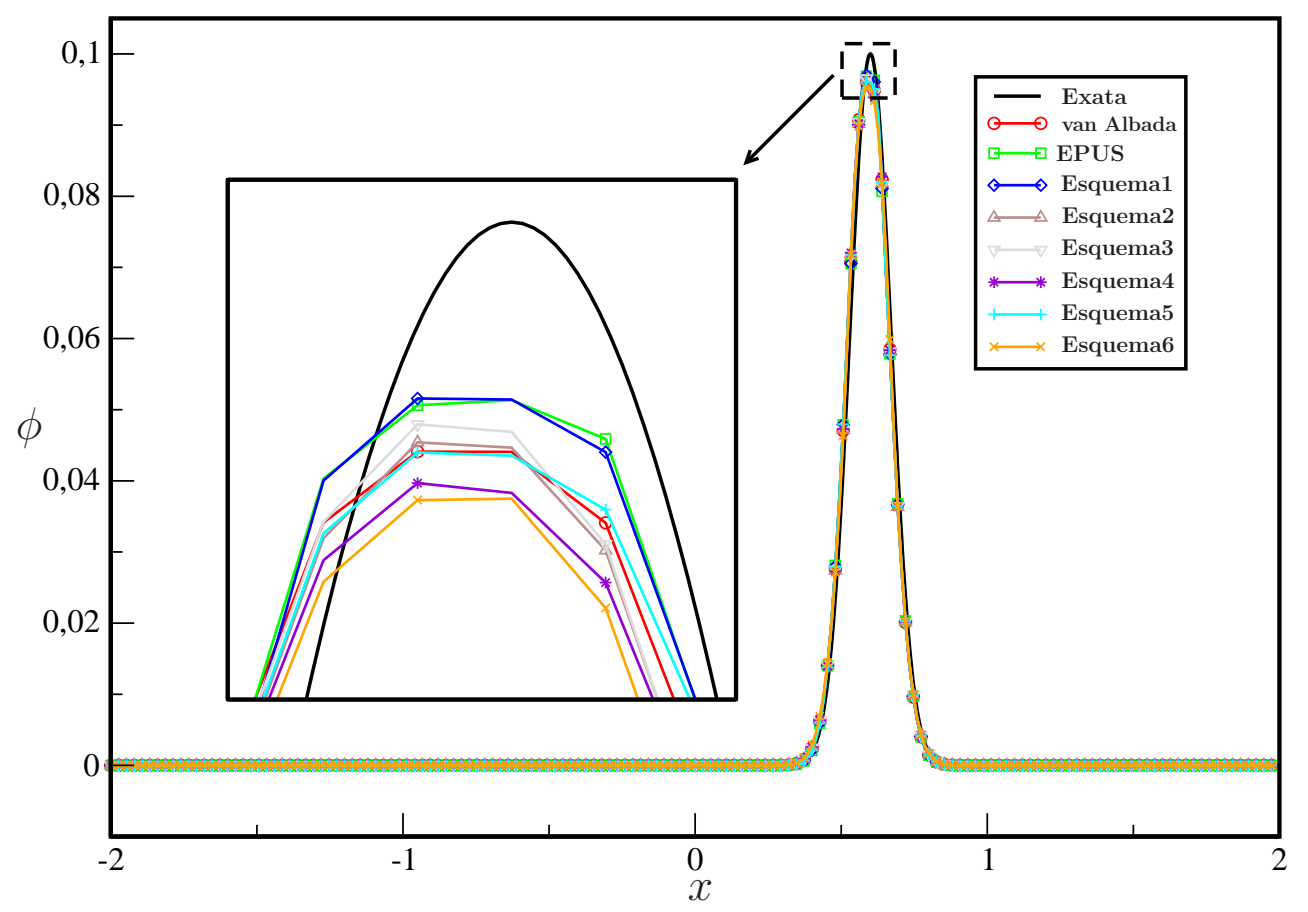

Figura 6.2: Solução exata e resultados numéricos (Esquemas 1-6 com os melhores parâmetros e referências van Albada e EPUS) para advecção de um escalar - Teste 1, mostrando ampliação da região de alto gradiente.

À partir da Figura 6.1, observa-se que o Esquema 1 fornece o melhor resultado para o caso em que $\beta=0.1545$. Os Esquemas 2, 3, 4 e 6 fornecem uma solução melhor para o caso $\epsilon=10^{-1}$. O Esquema 5 não apresentou mudanças significativas quanto a variação do parâmetro $\epsilon$. A Figura 6.2 mostra as comparações das melhores soluções numéricas (com respeito aos parâmetros $\beta$ e $\epsilon$ ), mais as soluções com os esquemas van Albada e EPUS, e a solução exata do problema. Vê-se por essa figura que o Esquema 1 apresentou o melhor resultado e mostrou ser bastante comparável ao esquema EPUS. Os Esquemas 2, 3 e 5 apresentaram também resultados satisfatórios, tendo o mesmo comportamento do van Albada.

A seguir faz-se uma análise quantitativa dos esquemas pela quantificação dos erros, nas normas $L_{1}, L_{2}$ e $L_{\infty}$, e extrai-se estimativas para a ordem de convergência $q$ e a constante do erro $C$. Para tanto, as seguintes definições são utilizadas.

$$
\begin{gathered}
\left\|E_{h}\right\|_{L_{1}}=\frac{\sum_{i=1}^{N}\left|\phi_{\text {exata }[i]}-\phi_{\text {numérica }[i]}\right|}{\sum_{i=1}^{N}\left|\phi_{\text {exata }[i]}\right|}, \\
\left\|E_{h}\right\|_{L_{2}}=\sqrt{\frac{\sum_{i=1}^{N}\left(\phi_{\text {exata }[i]}-\phi_{\text {numérica }[i]}\right)^{2}}{\sum_{i=1}^{N}\left(\phi_{\text {exata }[i]}\right)^{2}}},
\end{gathered}
$$




$$
\left\|E_{h}\right\|_{L_{\infty}}=\frac{\max _{1 \leq i \leq N}\left|\phi_{\text {exata }[i]}-\phi_{\text {numérica }[i]}\right|}{\max _{1 \leq i \leq N}\left|\phi_{\text {exata }[i]}\right|}
$$

onde $N$ é o número de pontos do domínio e o índice $h$ em $E_{h}$ corresponde ao espaçamento $\delta_{x}$ entre os pontos. A ordem de convergência dos métodos numéricos é estimada de maneira usual (ver, por exemplo, [36]) usando-se a fórmula

$$
\left\|E_{h}\right\| \approx C h^{q}+\text { termos de alta ordem. }
$$

Assumindo que a solução está no regime assintótico e que três tamanhos de malha satisfazem $h 2 / h 1=h 3 / h 2$, com $h 3<h 2<h 1$, a ordem observada é obtida como

$$
q=\frac{\log || E_{h 2}|| /\left\|E_{h 1}\right\|}{\log (h 2 / h 1)} .
$$

A constante do erro $C$ é estimada usando a expressão

$$
C \approx\left\|E_{h}\right\| / h^{q}
$$

As Tabelas 6.1 e 6.2 apresentam os erros e estimativas para ordem de convergência e constante do erro, quando os vários esquemas FUS-RF e os esquemas van Albada e EPUS são usados para resolver o problema. Pode-se observar por estas tabelas que os erros nas três normas $L_{1}$, $L_{2}$ e $L_{\infty}$ decrescem com o aumento do número de pontos de malha (refinamento de malha), indicando convergência (globalmente de primeira ordem ) com o uso dos esquemas selecionados. Observa-se também que a constante do erro para os esquemas Esquema 1, van Albada e EPUS são praticamente as mesmas; ao passo que os cálculos com os outros esquemas forneceram uma constante do erro maior (mantendo a mesma malha e a mesma ordem). A análise da precisão apresentada aqui parece indicar que o erro dominante é o da aproximação temporal.

O gráfico da constante de erro $C$ em função da malha $N$ é apresentado na Figura 6.3, onde nota-se que ao fazer o refinamento de malha, a constante do erro para todos os esquemas tendem à um valor constante, indicando que a solução numérica está no regime assintótico. E mais uma vez observa-se uma diferença substancial na constante do erro, quando se usa os esquemas van Albada, EPUS e o Esquema 1.

Teste 2: Neste segundo teste é transportada por advecção uma condição inicial com descontinuidades, as quais oferecem dificuldades aos esquema numéricos (ver Toro [45] ou Wei e Gu [52]). O problema é definido para valores de $x$ em [-1,1], e os seguintes dados foram utilizados: uma malha de 500 células computacionais; número de Courant $\theta=0.2$; e tempo final de simulação $T=0.2$. 
Tabela 6.1: Comparação dos erros nas normas $L_{1}, L_{2}$ e $L_{\infty}$ para o Teste 1 em várias malhas, mostrando os ordens observadas e as constantes do erro. Os resultados são para os esquemas van Albada, EPUS e os Esquemas 1-6 com os melhores parâmetros.

\begin{tabular}{|c|c|c|c|c|c|c|c|c|c|c|}
\hline & \multirow[t]{2}{*}{$\bar{N}$} & \multicolumn{3}{|c|}{$L_{1}$} & \multicolumn{3}{|c|}{$L_{2}$} & \multicolumn{3}{|c|}{$\overline{L_{\infty}}$} \\
\hline & & Erro & $q$ & $C$ & Erro & $q$ & $C$ & Erro & $q$ & $C$ \\
\hline Van & 20 & 0.584809 & - & - & 0.225778 & & - & 0.407075 & & \\
\hline \multirow[t]{7}{*}{ Albada } & 40 & 0.283698 & 1.0436 & 3.1366 & 0.067540 & 1.7410 & 3.7208 & 0.247954 & 0.7152 & 1.2870 \\
\hline & 80 & 0.148639 & 0.9325 & 2.4288 & 0.017431 & 1.9540 & 6.0757 & 0.144884 & 0.7751 & 1.4775 \\
\hline & 160 & 0.073407 & 1.0178 & 3.1358 & 0.004208 & 2.0503 & 8.1083 & 0.065060 & 1.1550 & 4.6107 \\
\hline & 320 & 0.036049 & 1.0259 & 3.2311 & 0.001009 & 2.0598 & 8.3974 & 0.026975 & 1.2701 & 7.0493 \\
\hline & 640 & 0.017861 & 1.0131 & 3.0550 & 0.000247 & 2.0293 & 7.3468 & 0.013415 & 1.0077 & 2.2325 \\
\hline & 1280 & 0.008873 & 1.0092 & 2.9955 & 0.000061 & 2.0116 & 6.7164 & 0.006702 & 1.0012 & 2.1596 \\
\hline & 2560 & 0.004421 & 1.0048 & 2.9197 & 0.000015 & 2.0043 & 6.4364 & 0.003350 & 1.0001 & 2.1463 \\
\hline \multirow[t]{8}{*}{ EPUS } & 20 & 0.584028 & - & - & 0.220115 & - & - & 0.389471 & - & - \\
\hline & 40 & 0.284844 & 1.0358 & 3.0936 & 0.068603 & 1.6819 & 3.2980 & 0.272993 & 0.5126 & 0.8888 \\
\hline & 80 & 0.146306 & 0.9611 & 2.6048 & 0.018823 & 1.8657 & 5.0360 & 0.143881 & 0.9239 & 2.2915 \\
\hline & 160 & 0.072756 & 1.0078 & 2.9957 & 0.004427 & 2.0880 & 9.8017 & 0.059244 & 1.2801 & 6.6600 \\
\hline & 320 & 0.035835 & 1.0216 & 3.1525 & 0.001053 & 2.0708 & 9.1978 & 0.028636 & 1.0488 & 2.8377 \\
\hline & 640 & 0.017817 & 1.0080 & 2.9700 & 0.000254 & 2.0486 & 8.3482 & 0.013835 & 1.0494 & 2.8454 \\
\hline & 1280 & 0.008873 & 1.0057 & 2.9354 & 0.000062 & 2.0288 & 7.5493 & 0.006808 & 1.0230 & 2.4879 \\
\hline & 2560 & 0.004423 & 1.0041 & 2.9084 & 0.000015 & 2.0156 & 6.9960 & 0.003377 & 1.0115 & 2.3282 \\
\hline \multirow{8}{*}{$\begin{array}{l}\text { Esq } 1 \\
\beta=0.1545\end{array}$} & 20 & 0.572588 & - & - & 0.216481 & - & - & 0.388652 & - & - \\
\hline & 40 & 0.280348 & 1.0302 & 3.0059 & 0.062838 & 1.7845 & 3.8259 & 0.255981 & 0.6024 & 1.0248 \\
\hline & 80 & 0.143467 & 0.9664 & 2.5953 & 0.017663 & 1.8309 & 4.2573 & 0.137096 & 0.9008 & 2.0372 \\
\hline & 160 & 0.072051 & 0.9936 & 2.8149 & 0.004330 & 2.0279 & 7.6834 & 0.060121 & 1.1892 & 4.8334 \\
\hline & 320 & 0.035684 & 1.0137 & 3.0319 & 0.001047 & 2.0477 & 8.2656 & 0.028635 & 1.0700 & 3.1142 \\
\hline & 640 & 0.017780 & 1.0049 & 2.9177 & 0.000 & 2.0418 & 8.0518 & 0.013835 & 1.0493 & 2.8445 \\
\hline & 1280 & 0.008 & 1.0038 & 2.9004 & 0.000062 & 2.0271 & 7.4759 & 0.006808 & 1.0230 & 2.4883 \\
\hline & 2560 & 0.004424 & 1.0030 & 2.8877 & 0.000015 & 2.0154 & 6.9844 & 0.003377 & 1.0115 & 2.3285 \\
\hline \multirow{8}{*}{$\begin{array}{l}\text { Esq 2 } \\
\epsilon=0.1\end{array}$} & 20 & 1.125584 & - & - & 0.622912 & - & - & 0.719825 & - & - \\
\hline & 40 & 0.651442 & 0.7889 & 6.9237 & 0.272877 & 1.1907 & 9.6650 & 0.421198 & 0.7731 & 4.2694 \\
\hline & 80 & 0.310524 & 1.0689 & 16.017 & 0.082374 & 1.7279 & 48.320 & 0.270361 & 0.6396 & 2.8618 \\
\hline & 160 & 0.154502 & 1.0070 & 12.749 & 0.019602 & 2.0711 & 171.33 & 0.147882 & 0.8704 & 6.7055 \\
\hline & 320 & 0.075 & 1.0382 & 14.613 & 0.00 & 2.0874 & 184.03 & 0.07 & 1.0292 & 13.445 \\
\hline & 640 & 0.036684 & 1.0361 & 14.464 & 0.001072 & 2.1052 & 201.44 & 0.028375 & 1.3525 & 69.378 \\
\hline & 1280 & 0.018030 & 1.0247 & 13.541 & 0.000256 & 2.0648 & 159.54 & 0.013799 & 1.0400 & 211.44 \\
\hline & 2560 & 0.008924 & 1.0145 & 12.676 & 0.000062 & 2.0345 & 131.22 & 0.006793 & 1.0223 & 10.202 \\
\hline \multirow{8}{*}{$\begin{array}{l}\text { Esq 3 } \\
\epsilon=0.1\end{array}$} & 20 & 1.137281 & - & - & 0.630589 & - & - & 0.722403 & - & - \\
\hline & 40 & 0.673677 & 0.7554 & 6.4763 & 0.292848 & 1.1065 & 8.0591 & 0.430972 & 0.7452 & 4.0178 \\
\hline & 80 & & 1.0716 & 16.700 & & 1.7393 & 53.647 & 0.28 & 0.6163 & 2.7308 \\
\hline & 160 & & 1.0481 & 15.3 & & 2.1274 & 224.59 & & 0.8997 & 7.7699 \\
\hline & 320 & 0.075306 & 1.0414 & 14.868 & 0.004585 & 2.1302 & 227.30 & 0.070769 & 1.0902 & 17.904 \\
\hline & 640 & 0.036602 & 1.0408 & 14.823 & 0.001059 & 2.1132 & 208.55 & 0.028001 & 1.3376 & 62.818 \\
\hline & 1280 & 0.018004 & 1.0236 & 13.421 & 0.000253 & 2.0609 & 154.27 & 0.013684 & 1.0329 & 10.837 \\
\hline & 2560 & 0.008914 & 1.0140 & 12.618 & 0.000062 & 2.0297 & 126.04 & 0.006767 & 1.0157 & 9.6984 \\
\hline \multirow{8}{*}{$\begin{array}{l}\text { Esq } 4 \\
\epsilon=0.1\end{array}$} & 20 & 1.114156 & - & - & 0.626132 & - & - & 0.724268 & - & - \\
\hline & 40 & 0.650327 & 0.7767 & 6.6628 & 0.275454 & 1.1846 & 9.5790 & 0.441556 & 0.7139 & 3.7482 \\
\hline & 80 & 0.305981 & 1.0877 & 16.915 & & 1.8069 & 61.802 & 0.257132 & 0.7800 & 4.5698 \\
\hline & 160 & 0.154477 & 0.9860 & 11.625 & 0.018776 & 2.0677 & 161.74 & 0.147127 & 0.8054 & 5.0180 \\
\hline & 320 & 0.0749 & 1.0434 & 14.948 & 0.004612 & 2.0254 & 134.36 & 0.073677 & 0.9977 & 11.655 \\
\hline & 640 & 0.036694 & 1.0303 & 13.987 & 0.001081 & 2.0919 & 188.31 & 0.028610 & 1.3646 & 75.035 \\
\hline & 1280 & 0.018040 & 1.0243 & 13.509 & 0.000258 & 2.0670 & 163.09 & 0.013852 & 1.0463 & 11.964 \\
\hline & 2560 & 0.008941 & 1.0127 & 12.536 & 0.000062 & 2.0391 & 136.17 & 0.006806 & 1.0251 & 10.428 \\
\hline
\end{tabular}


Tabela 6.2: Continuação da Tabela 6.1.

\begin{tabular}{l|r|cc|c|c|c|c|c|c|c|c}
\hline \hline & $N$ & \multicolumn{4}{|c|}{$L_{1}$} & \multicolumn{4}{c|}{$L_{2}$} & \multicolumn{3}{|c}{$L_{\infty}$} \\
\cline { 3 - 11 } & & Erro & $q$ & $C$ & Erro & $q$ & $C$ & Erro & $q$ & $C$ \\
\hline \hline Esq 5 & 20 & 1.106949 & - & - & 0.623336 & - & - & 0.724062 & - & - \\
$\epsilon=0$ & 40 & 0.647287 & 0.7741 & 6.5802 & 0.270362 & 1.2051 & 9.9962 & 0.435558 & 0.7332 & 3.9176 \\
& 80 & 0.304438 & 1.0882 & 16.863 & 0.078552 & 1.7831 & 56.480 & 0.273676 & 0.6703 & 3.2452 \\
& 160 & 0.152903 & 0.9935 & 11.890 & 0.020682 & 1.9252 & 95.390 & 0.157584 & 0.7963 & 5.1645 \\
& 320 & 0.074601 & 1.0353 & 14.281 & 0.004795 & 2.1086 & 213.07 & 0.070006 & 1.1705 & 26.619 \\
& 640 & 0.036417 & 1.0345 & 14.226 & 0.001105 & 2.1167 & 222.04 & 0.029465 & 1.2484 & 39.527 \\
& 1280 & 0.018016 & 1.0152 & 12.726 & 0.000261 & 2.0803 & 179.97 & 0.014049 & 1.0684 & 13.997 \\
& 2560 & 0.008938 & 1.0112 & 12.401 & 0.000063 & 2.0472 & 145.32 & 0.006857 & 1.0347 & 11.253 \\
\hline Esq 6 & 20 & 1.060884 & - & - & 0.570793 & - & - & 0.696716 & - & - \\
$\epsilon=0.1$ & 40 & 0.584963 & 0.8588 & 7.6650 & 0.227461 & 1.3273 & 12.129 & 0.410652 & 0.7626 & 4.0337 \\
& 80 & 0.286833 & 1.0281 & 12.728 & 0.069793 & 1.7044 & 37.537 & 0.234275 & 0.8097 & 4.6444 \\
& 160 & 0.152095 & 0.9152 & 8.3924 & 0.017169 & 2.0232 & 121.67 & 0.126944 & 0.8840 & 6.1087 \\
& 320 & 0.074339 & 1.0327 & 14.046 & 0.004316 & 1.9918 & 106.01 & 0.072913 & 0.7999 & 4.2265 \\
& 640 & 0.036669 & 1.0195 & 13.134 & 0.001054 & 2.0335 & 131.00 & 0.028889 & 1.3356 & 64.074 \\
& 1280 & 0.018060 & 1.0217 & 13.303 & 0.000255 & 2.0423 & 137.80 & 0.013806 & 1.0652 & 13.468 \\
& 2560 & 0.008939 & 1.0145 & 12.700 & 0.000062 & 2.0299 & 127.25 & 0.006790 & 1.0236 & 10.293 \\
\hline \hline
\end{tabular}

(a) $L_{1}$

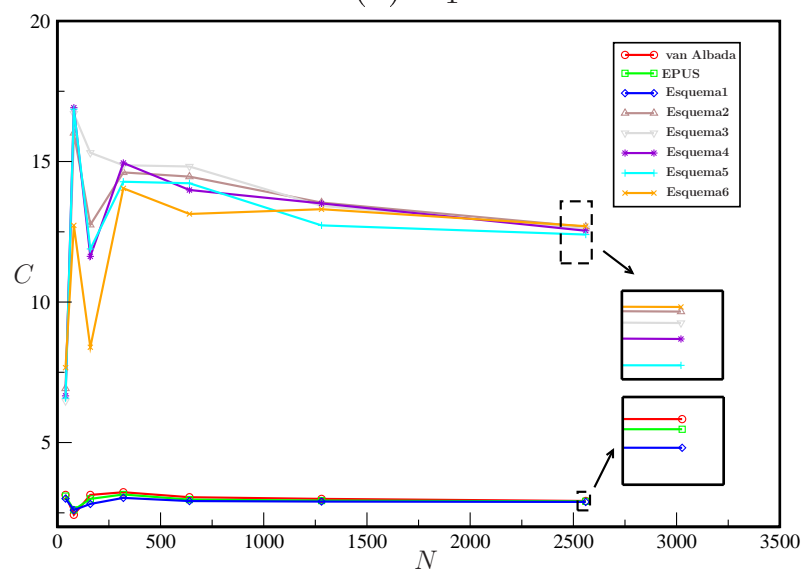

(b) $L_{2}$

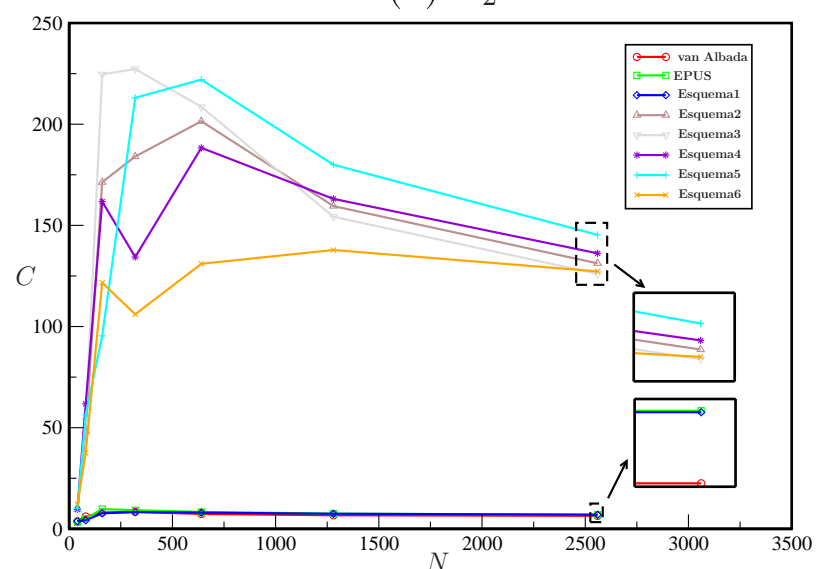

(c) $L_{\infty}$

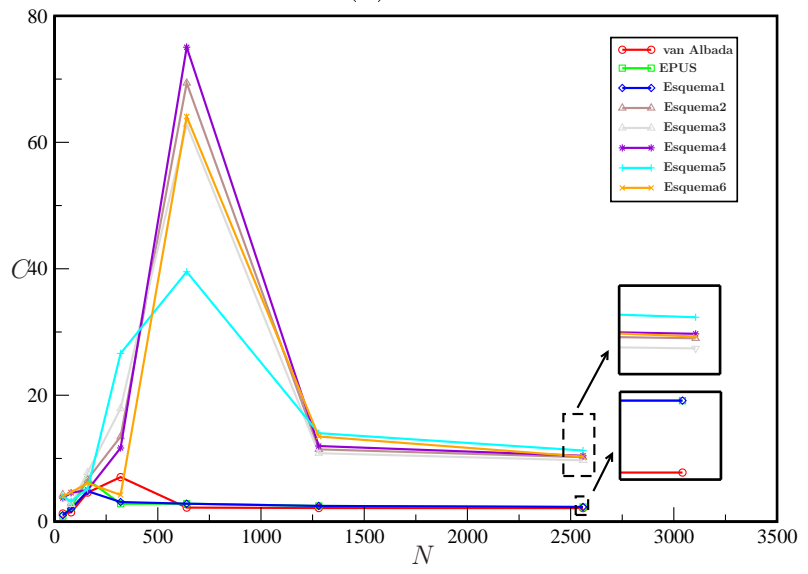

Figura 6.3: Constante do erro para os esquemas van Albada, EPUS e Esquemas 1-6 com os melhores parâmetros, aplicados ao Teste 1 . 
A condição inicial escolhida é

$$
\phi_{0}(x)= \begin{cases}-\operatorname{sen}(\pi)+\frac{7}{10} x, & -0.9 \leq x<0, \\ 1, & 0 \leq x \leq 0.2, \\ 4 x-\frac{3}{5}, & 0.2<x \leq 0.4, \\ -4 x+\frac{13}{5}, & 0.4<x \leq 0.6, \\ 1, & 0.6<x \leq 0.8, \\ 0, & \text { caso contrário. }\end{cases}
$$

Os esquemas FUS-RF foram testados nesse problema, com solução inicial descontínua, usando-se os mesmos valores das constantes $\beta$ e $\epsilon$ usados no problema Teste $\mathbf{1}$.

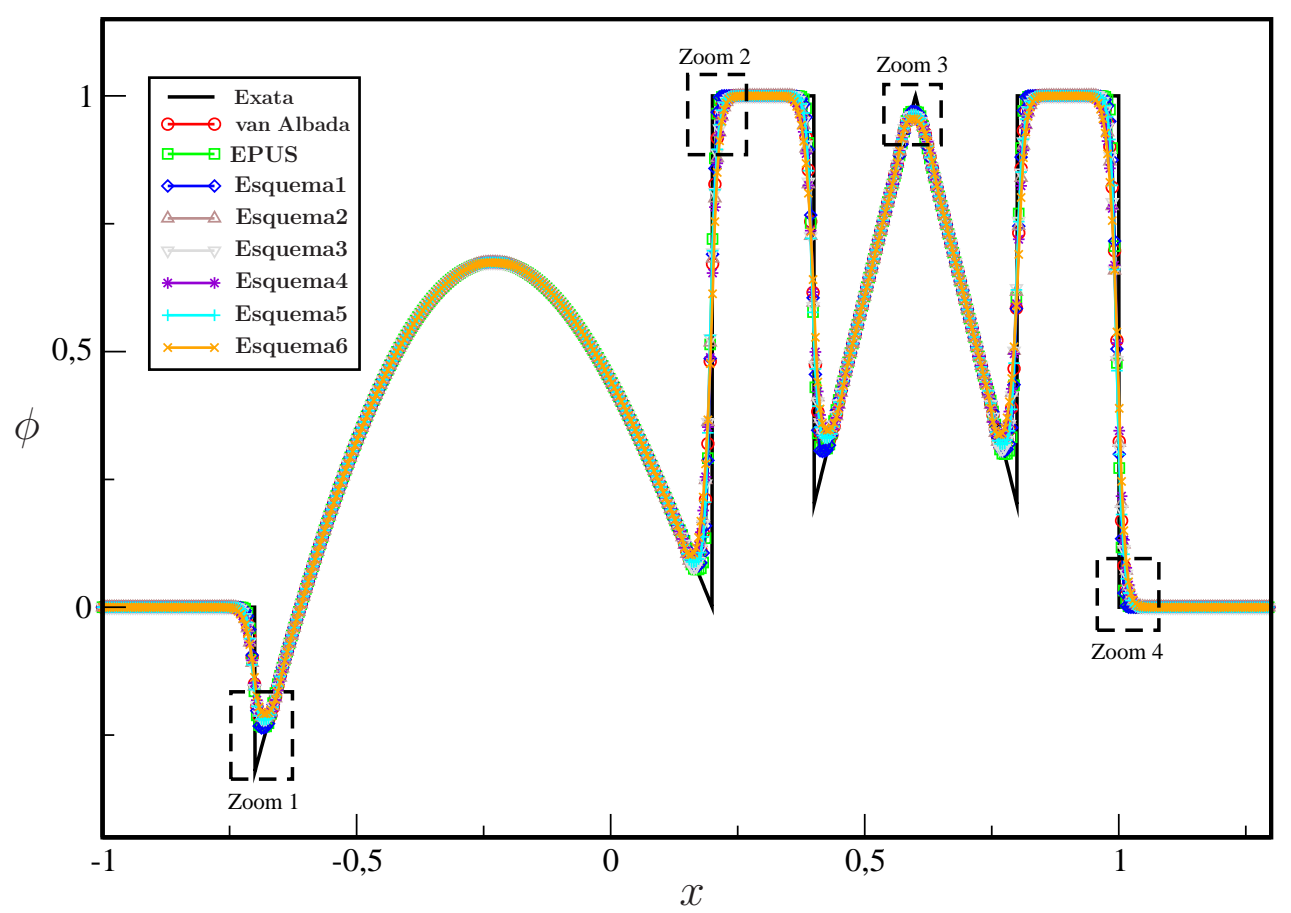

Figura 6.4: Solução exata e resultados numéricos (Esquemas 1-6 com os melhores parâmetros e referências van Albada e EPUS) para advecção de um escalar - Teste 2, mostrando regiões de ampliações (Zoom 1-4).

A Figura 6.4 apresenta as melhores soluções numéricas com respeito aos parâmetros $\beta$ e $\epsilon$ $(\beta=0.1545$ para o Esquema $1, \epsilon=0.1$ para os Esquemas 2, 3, 4 e 6 e $\epsilon=0.01$ para o Esquema $5)$, mais as soluções com os esquemas van Albada e EPUS, em comparação com solução exata. Vê-se que nenhum dos esquemas estudados conseguiu aproximar de maneira correta a solução exata em virtude das descontinuidades. Na Figura 6.5 apresenta-se as ampliações $(Z o o m)$ destacadas na Figura 6.4, onde pode-se observar mais detalhadamente os resultados numéricos e verificar (qualitativamente) o erro cometido. Observa-se também que os picos e vales não foram bem resolvidos com o uso dos esquemas; porém percebe-se que o Esquema 1 e o esquema 
EPUS resolveram melhor esses extremos.

(a) Zoom 1

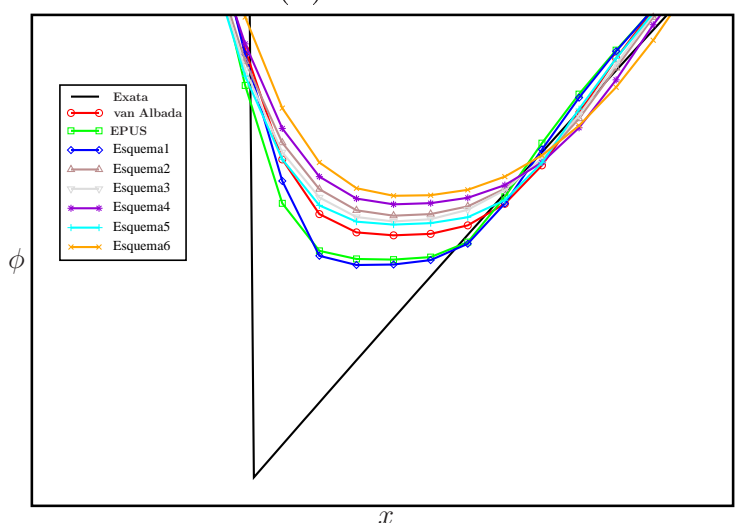

(c) Zoom 3

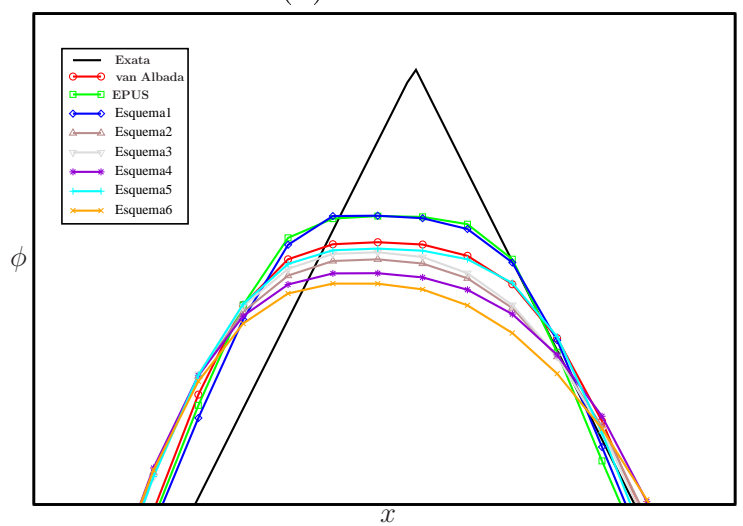

(b) Zoom 2

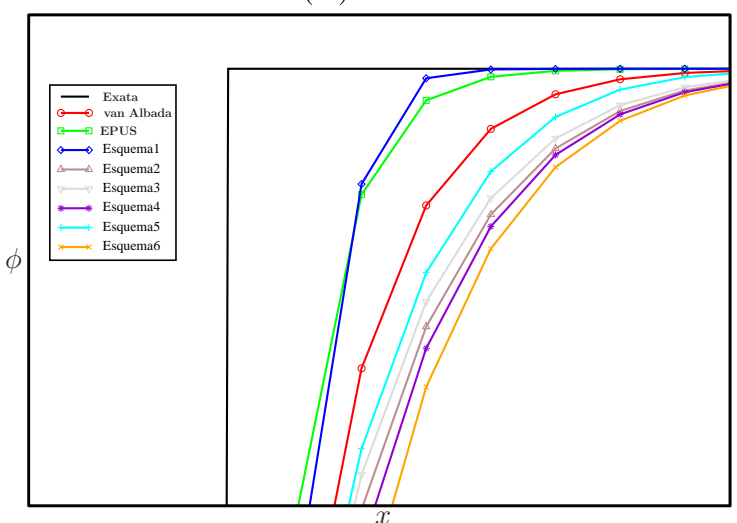

(d) Zoom 4

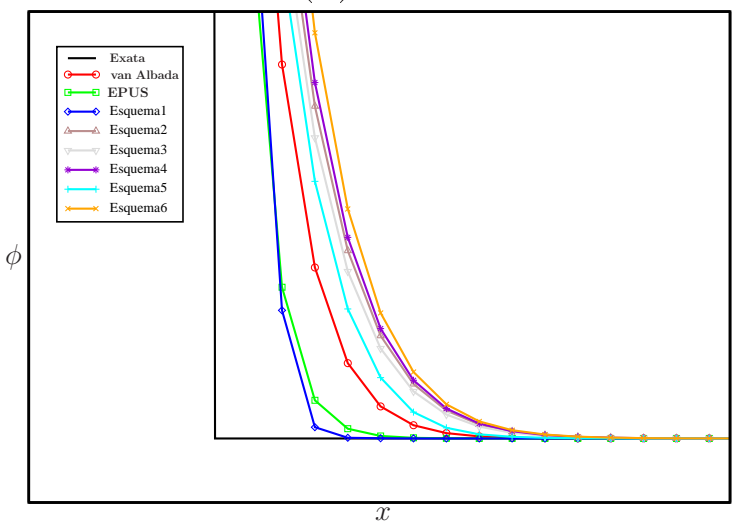

Figura 6.5: Continuação da Figura 6.4.

A Figura 6.6 apresenta a variação total TV em função do tempo $t$ usando a malha de 500 pontos. É possível notar que todos os esquemas satisfazem este conceito de estabilidade quando se avança no tempo, pois a TV da solução decresce para todos os esquemas da classe FUS-RF e também para os esquemas van Albada e EPUS.

\subsection{Resultados para equação de Burgers}

O desempenho dos esquemas upwind estudados neste trabalho é investigado agora para o caso da equação não linear de Burgers (2.5), nos casos sem viscosidade $(\nu=0)$ ou com o coeficiente de viscosidade $\nu=0.05$ (caso viscoso).

Teste 3 (caso não viscoso): Neste teste a condição inicial adotada é suave e dada por

$$
\phi_{0}(x)=1+\frac{1}{2} \sin (\pi x), \quad x \in[-1,1] .
$$

A solução exata deste problema é dada (implicitamente) como (ver [43]) 


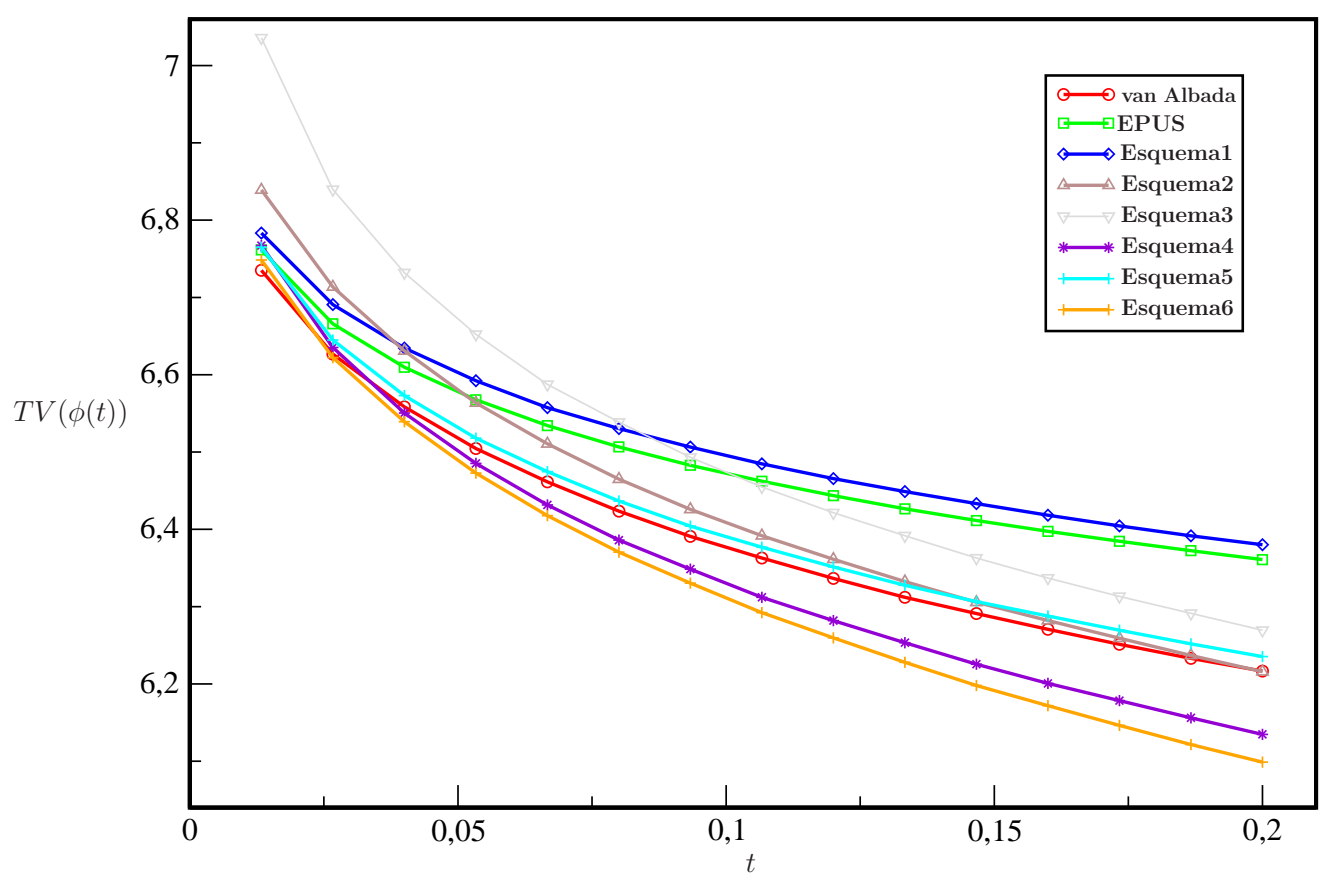

Figura 6.6: Variação total para o Teste 2 com respeito ao tempo.

$$
\phi(x, t)=1+\frac{1}{2} \sin (\pi(x-\phi t)), \quad x \in[-1,1] .
$$

Neste problema não linear, a condição inicial suave evolui para uma descontinuidade (choque), como mostrado na Figura (6.7) usando o limitador MC (Monotonized Central) de LeVeque [21].

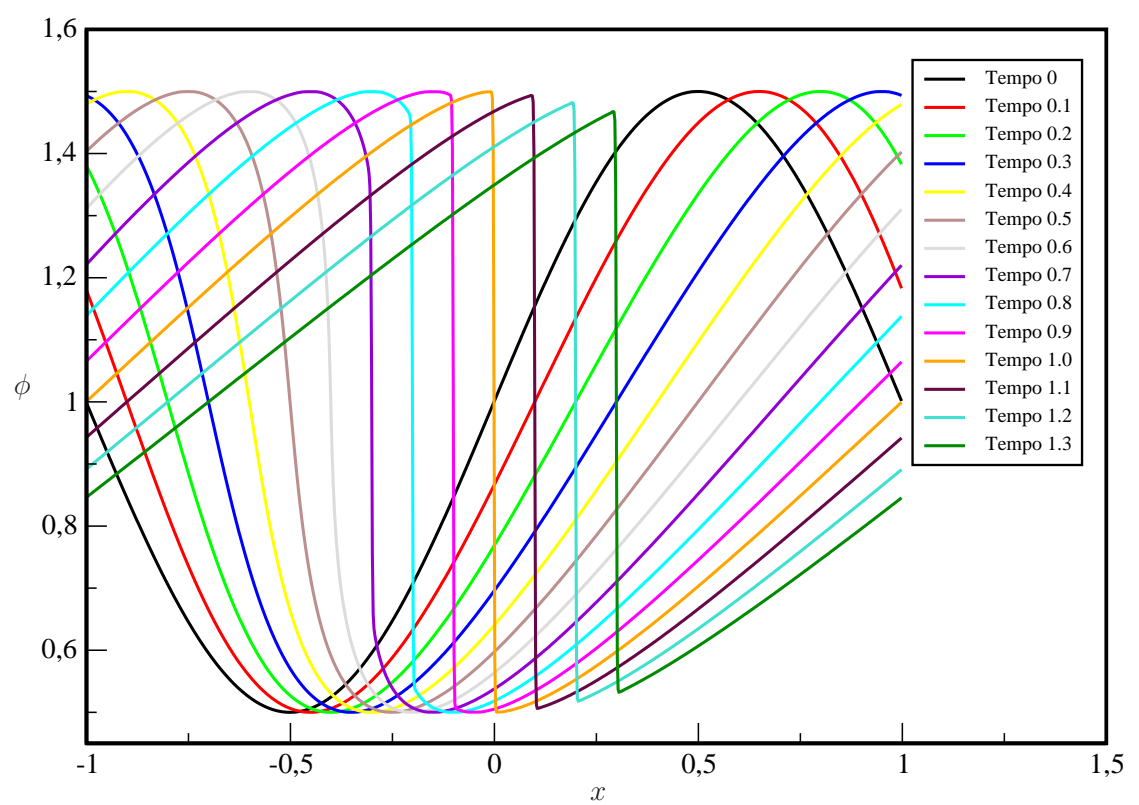

Figura 6.7: Evolução no tempo da solução numérica para equação de Burgers usando o esquema MC. 
Da mesma forma como foi feito para advecção de escalares, os esquemas FUS-RF são testados com diversos valores de $\beta$ (ver Teste $\mathbf{1}$ ) e para os valores de $\epsilon$ variando de 0 à $10^{-2}$. A Figura 6.8 exibe a solução encontrada pelos novos esquemas antes (no tempo $t=0.5$ ) e depois $(t=1.5)$ do choque. Os Esquemas 2 e 3 apresentaram ligeiras oscilações não físicas no ponto $x=0.5$ para o caso $\epsilon=0.1$ (isto já era de se esperar uma vez que, para este valor de $\epsilon$, os esquemas não são TVD); porém para outros valores de $\epsilon$ os cálculos são livres de oscilações. $\mathrm{Na}$ Figura 6.9 estão comparados os resultados numéricos com os vários esquemas e a solução exata. Nas regiões de ampliação (ver Figura 6.10), nota-se que antes do choque (solução suave) todos os esquemas fornecem resultados semelhantes, comparando-se (no global) com a solução exata. No entanto, próximo ao choque, todos os esquemas numéricos apresentaram um certo grau de dissipação, com destaque para a região 3, onde o esquema EPUS aproximou-se mais da solução exata. Na região 4, o Esquema 1 foi o que mais se aproximou da solução exata.

O erro, a ordem observada e a constante do erro (na $L_{2}$, antes e depois do choque) para esse problema utilizando-se 7 refinamentos $(\operatorname{com} h 2 / h 1=h 3 / h 2=h 4 / h 3=h 5 / h 4=h 6 / h 5=$ $h 7 / h 6=2)$ e três valores dos parâmetros $\beta$ e $\epsilon(\beta=0.1545,0.1605$ e $0.1670 ; \epsilon=0,0.1$ e 0.01 , parâmetros estes selecionados dos melhores resultados obtidos na Figura 6.8) estão mostrados nas Tabelas 6.3-6.5. Observa-se claramente por essas tabelas que as ordens antes do choque atingiram o valor $\approx 2$ para todos os esquemas; e depois do choque $\approx 1$. A constante do erro teve comportamento análogo. A Figura 6.11 ilustra o comportamento da constante do erro na norma $L_{1}$ para as soluções antes e depois do choque. O Esquema 1 apresentou a menor constante do erro em ambas simulações.

Teste 4 (caso viscoso): Neste teste considera-se a equação de Burgers para $x \in[-2,3]$ com viscosidade $\nu=0.05$. A condição inicial, proposta por LeVeque (ver [21]), é dada por

$$
\phi_{0}(y)= \begin{cases}1, & y<-20 \\ \frac{1}{2}(1-\tanh y), & -20 \leq y \leq 20 \\ 0, & y>20\end{cases}
$$

onde $y=\frac{x}{4 \nu}$. A solução exata deste problema é

$$
\phi(x)=1-\frac{1}{2}\left(1-\tanh \left(\frac{-x+0.5 t}{4 \nu}\right)\right) .
$$

O problema foi resolvido usando-se todos os esquemas estudados anteriormente a $C F L=0.9$, tempo final $T=1.0$, com os parâmetros $\beta=0.1545 ; \epsilon=0.1$ para os Esquemas 2, 3 e 6; $\epsilon=0.01$ para o Esquema 4; e $\epsilon=0$ para o esquema 5. As Figuras 6.12 e 6.13 mostram as soluções numéricas comparadas com a solução exata, onde pode-se ver que, no geral, os esquemas aproximam-se bem da solução exata, com o EPUS e o Esquema 1 fornecendo os melhores resultados. 
(a) Esquema 1

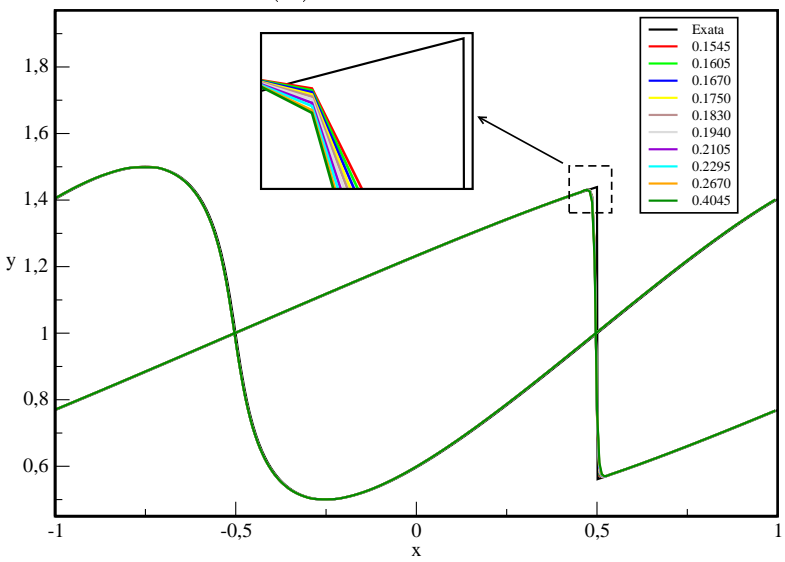

(c) Esquema 3

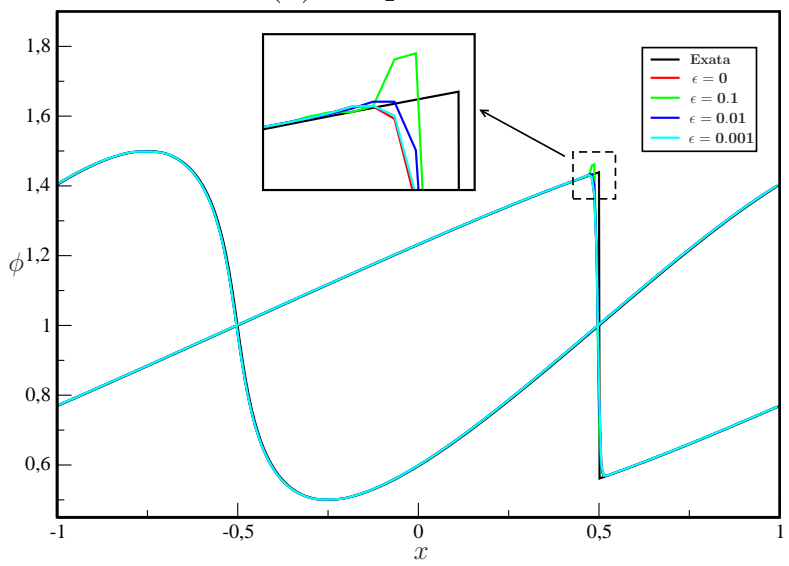

(e) Esquema 5

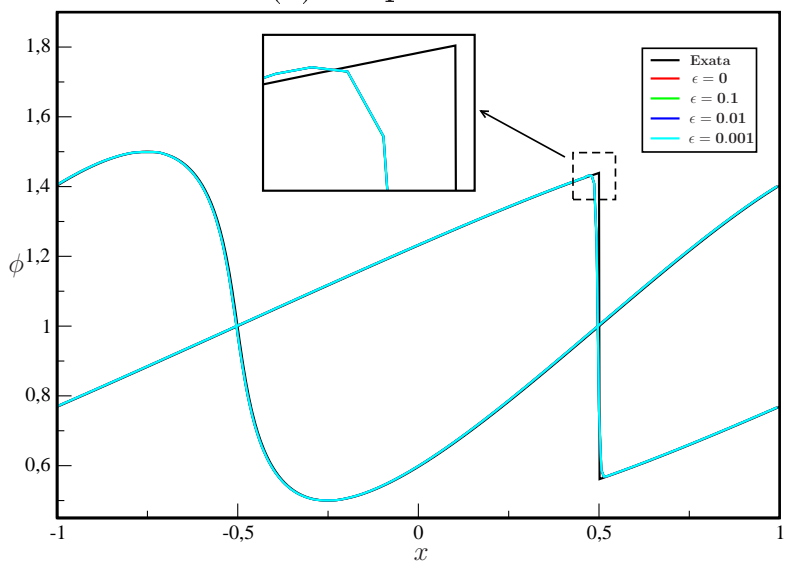

(b) Esquema 2

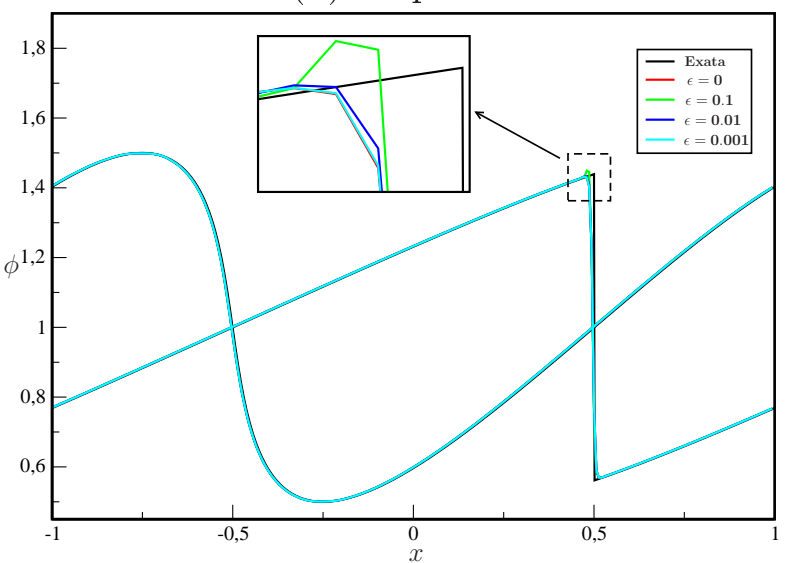

(d) Esquema 4

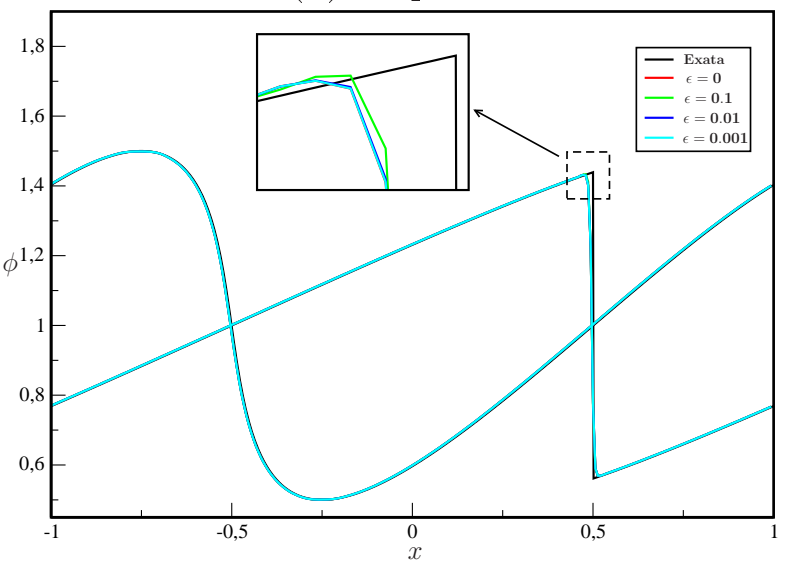

(f) Esquema 6

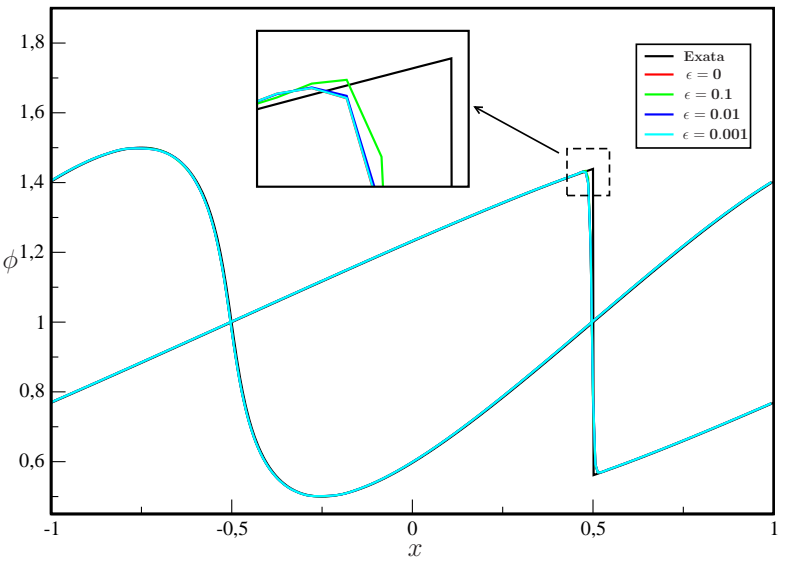

Figura 6.8: Solução exata e resultados obtidos com os esquemas FUS-RF para equação de Burgers. (a) Esquema 1 utilizando vários valores do parâmetro $\beta$; (b)-(f) Esquemas $2-6$ utilizando vários valores do parâmetro $\epsilon$. 


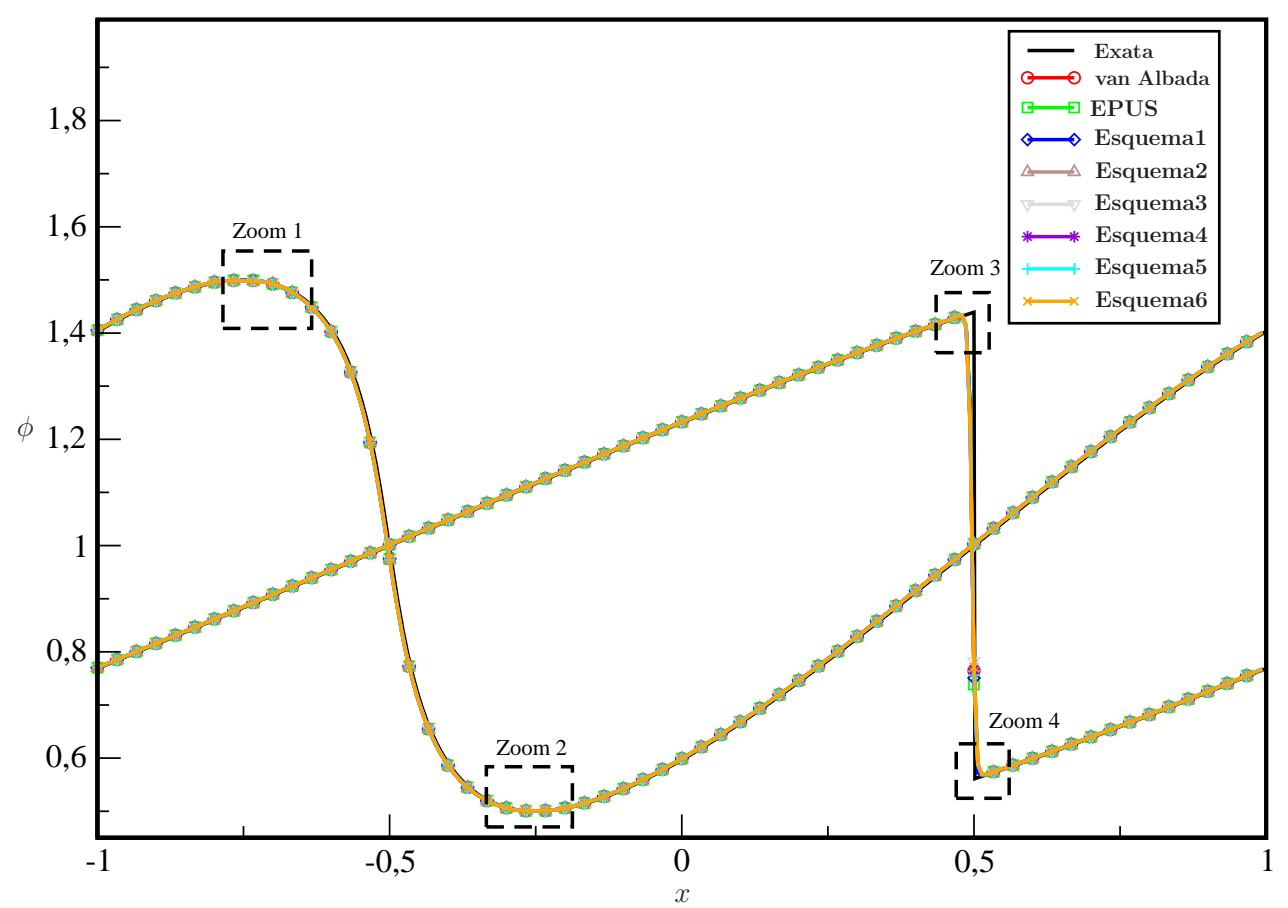

Figura 6.9: Comparação (antes e depois do choque) entre a solução exata e as soluções numéricos (Esquemas 1-6 com os melhores parâmetros e referências van Albada e EPUS) para equação de Burgers - Teste 3, mostrando regiões de ampliação.

(a) Zoom 1

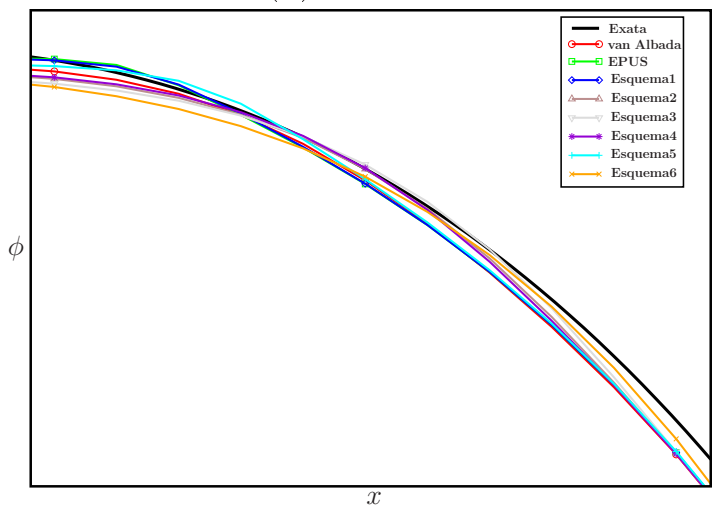

(c) Zoom 3

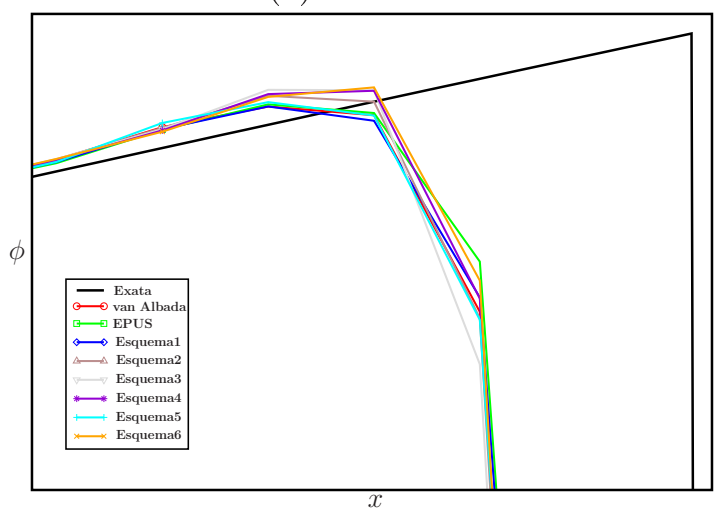

(b) Zoom 2

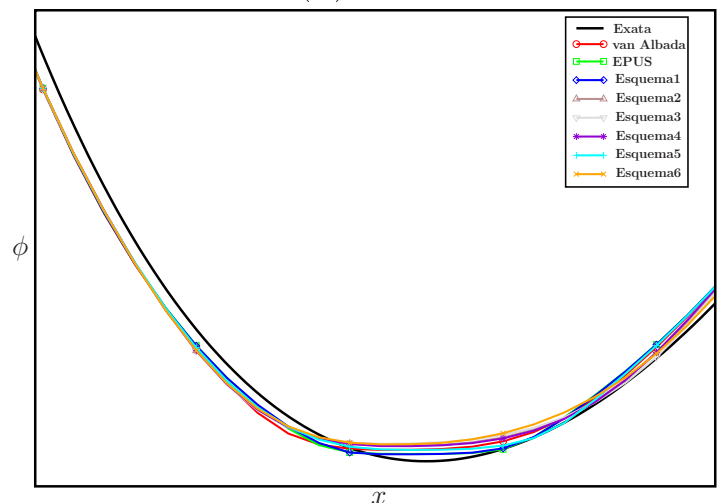

(d) Zoom 4

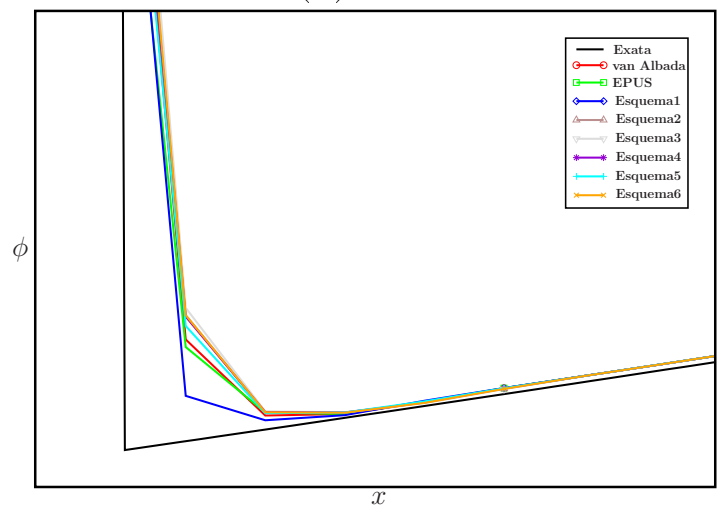

Figura 6.10: Continuação da Figura 6.9. 
Tabela 6.3: Comparação dos erros na norma $L_{2}$ para o Teste 3 em várias malhas, mostrando (antes e depois do choque) as ordens observadas e as constantes do erro. Os resultados são para os esquemas van Albada, EPUS e os Esquemas 1-6 com vários parâmetros.

\begin{tabular}{|c|c|c|c|c|c|c|c|}
\hline \multirow[t]{2}{*}{ Esquema } & \multirow[t]{2}{*}{$N$} & \multicolumn{3}{|c|}{ Antes do choque } & \multicolumn{3}{|c|}{ Depois do choque } \\
\hline & & Erro & $q$ & $C$ & Erro & $q$ & $C$ \\
\hline \multirow[t]{7}{*}{ van Albada } & 20 & 0.00331413 & - & - & 0.02208255 & - & - \\
\hline & 40 & 0.00073411 & 2.174548 & 0.4953 & 0.01135879 & 0.959097 & 0.2009 \\
\hline & 80 & 0.00017569 & 2.062980 & 0.3546 & 0.00570734 & 0.992919 & 0.2224 \\
\hline & 160 & 0.00004325 & 2.022124 & 0.3050 & 0.00286341 & 0.995083 & 0.2241 \\
\hline & 320 & 0.00001075 & 2.008354 & 0.2871 & 0.00144286 & 0.988801 & 0.2181 \\
\hline & 640 & 0.00000268 & 2.003258 & 0.2798 & 0.00071998 & 1.002895 & 0.2342 \\
\hline & 1280 & 0.00000066 & 2.001334 & 0.2767 & 0.00036177 & 0.992857 & 0.2210 \\
\hline \multirow[t]{7}{*}{$\overline{\mathrm{EPUS}}$} & 20 & 0.00326155 & - & - & 0.02345930 & - & - \\
\hline & 40 & 0.00074417 & 2.131848 & 0.4418 & 0.01195722 & 0.972277 & 0.2200 \\
\hline & 80 & 0.00017846 & 2.060024 & 0.3563 & 0.00601381 & 0.991532 & 0.2331 \\
\hline & 160 & 0.00004362 & 2.032292 & 0.3216 & 0.00303915 & 0.984608 & 0.2272 \\
\hline & 320 & 0.00001080 & 2.013759 & 0.2965 & 0.00152271 & 0.997029 & 0.2399 \\
\hline & 640 & 0.00000268 & 2.006420 & 0.2857 & 0.00075866 & 1.005103 & 0.2500 \\
\hline & 1280 & 0.00000067 & 2.003130 & 0.2803 & 0.00038065 & 0.995002 & 0.2358 \\
\hline \multirow{7}{*}{$\begin{array}{l}\text { Esq1 } \\
\beta=0.1545\end{array}$} & 20 & 0.00319855 & - & - & 0.02265672 & - & - \\
\hline & 40 & 0.00073447 & 2.122625 & 0.4242 & 0.01158606 & 0.967549 & 0.2102 \\
\hline & 80 & 0.00017777 & 2.046659 & 0.3378 & 0.00582414 & 0.992271 & 0.2264 \\
\hline & 160 & 0.00004359 & 2.027804 & 0.3151 & 0.00292539 & 0.993418 & 0.2273 \\
\hline & 320 & 0.00001080 & 2.012873 & 0.2952 & 0.00147186 & 0.990983 & 0.2249 \\
\hline & 640 & 0.00000268 & 2.006257 & 0.2854 & 0.00072976 & 1.012143 & 0.2504 \\
\hline & 1280 & 0.00000067 & 2.003108 & 0.2803 & 0.00036743 & 0.989923 & 0.2203 \\
\hline \multirow{7}{*}{$\begin{array}{l}\text { Esq1 } \\
\beta=0.1605\end{array}$} & 20 & 0.00324089 & - & - & 0.02261251 & - & - \\
\hline & 40 & 0.00073675 & 2.137134 & 0.4444 & 0.01156819 & 0.966958 & 0.2095 \\
\hline & 80 & 0.00017793 & 2.049850 & 0.3421 & 0.00580149 & 0.995667 & 0.2283 \\
\hline & 160 & 0.00004361 & 2.028476 & 0.3162 & 0.00290560 & 0.997588 & 0.2300 \\
\hline & 320 & 0.00001080 & 2.013248 & 0.2958 & 0.00146239 & 0.990503 & 0.2229 \\
\hline & 640 & 0.00000268 & 2.006406 & 0.2857 & 0.00072585 & 1.010573 & 0.2468 \\
\hline & 1280 & 0.00000067 & 2.003158 & 0.2804 & 0.00036546 & 0.989940 & 0.2191 \\
\hline \multirow{7}{*}{$\begin{array}{l}\text { Esq1 } \\
\beta=0.1670\end{array}$} & 20 & 0.00328745 & - & - & 0.02259870 & - & - \\
\hline & 40 & 0.00073988 & 2.151606 & 0.4660 & 0.01156253 & 0.966782 & 0.2093 \\
\hline & 80 & 0.00017816 & 2.054076 & 0.3479 & 0.00578391 & 0.999340 & 0.2307 \\
\hline & 160 & 0.00004363 & 2.029553 & 0.3178 & 0.00289012 & 1.000917 & 0.2321 \\
\hline & 320 & 0.00001080 & 2.013754 & 0.2966 & 0.0014530 & 0.992003 & 0.2232 \\
\hline & 640 & 0.00000268 & 2.006602 & 0.2860 & 0.00072286 & 1.007325 & 0.2413 \\
\hline & 1280 & 0.00000067 & 2.003227 & 0.2805 & 0.00036396 & 0.989929 & 0.2182 \\
\hline \multirow{7}{*}{$\begin{array}{l}\text { Esq2 } \\
\epsilon=0\end{array}$} & 20 & 0.00360485 & - & - & 0.02207070 & - & - \\
\hline & 40 & 0.00077554 & 2.216656 & 0.5936 & 0.01133594 & 0.961228 & 0.2018 \\
\hline & 80 & 0.00017965 & 2.109999 & 0.4313 & 0.00565460 & 1.003405 & 0.2290 \\
\hline & 160 & 0.00004381 & 2.035837 & 0.3280 & 0.00281708 & 1.005227 & 0.2305 \\
\hline & 320 & 0.00001082 & 2.016578 & 0.3015 & 0.00140630 & 1.002293 & 0.2276 \\
\hline & 640 & 0.00000269 & 2.008159 & 0.2889 & 0.00070248 & 1.001370 & 0.2265 \\
\hline & 1280 & 0.00000067 & 2.003996 & 0.2820 & 0.00035155 & 0.998701 & 0.2231 \\
\hline \multirow{7}{*}{$\begin{array}{l}\text { Esq2 } \\
\epsilon=0.1\end{array}$} & 20 & 0.00352865 & - & - & 0.02512272 & - & - \\
\hline & 40 & 0.00076433 & 2.216656 & 0.5943 & 0.01287012 & 0.964967 & 0.2317 \\
\hline & 80 & 0.00017914 & 2.109985 & 0.4289 & 0.00638589 & 1.011064 & 0.2660 \\
\hline & 160 & 0.00004370 & 2.035821 & 0.3204 & 0.00318906 & 1.001754 & 0.2570 \\
\hline & 320 & 0.00001081 & 2.016578 & 0.3108 & 0.00159208 & 1.002222 & 0.2576 \\
\hline & 640 & 0.00000268 & 2.008545 & 0.2890 & 0.00079617 & 0.999749 & 0.2544 \\
\hline & 1280 & 0.00000067 & 2.003891 & 0.2816 & 0.00039755 & 1.001928 & 0.2576 \\
\hline
\end{tabular}


Tabela 6.4: Continuação da Tabela 6.3.

\begin{tabular}{|c|c|c|c|c|c|c|c|}
\hline \multirow[t]{2}{*}{ Esquema } & \multirow[t]{2}{*}{$N$} & \multicolumn{3}{|c|}{ Antes do choque } & \multicolumn{3}{|c|}{ Depois do choque } \\
\hline & & Erro & $q$ & $C$ & Erro & $q$ & $C$ \\
\hline Esq2 & 20 & 0.00358347 & & - & 0.02256244 & & \\
\hline \multirow[t]{6}{*}{$\epsilon=0.01$} & 40 & 0.00077290 & 2.213000 & 0.5852 & 0.01162179 & 0.957090 & 0.2043 \\
\hline & 80 & 0.00017954 & 2.105926 & 0.4246 & 0.00578521 & 1.006392 & 0.2369 \\
\hline & 160 & 0.00004379 & 2.035630 & 0.3276 & 0.00287777 & 1.007415 & 0.2378 \\
\hline & 320 & 0.00001082 & 2.016286 & 0.3010 & 0.00144024 & 0.998641 & 0.2288 \\
\hline & 640 & 0.00000269 & 2.007975 & 0.2885 & 0.00071767 & 1.004907 & 0.2362 \\
\hline & 1280 & 0.00000067 & 2.003906 & 0.2818 & 0.00035891 & 0.999675 & 0.2292 \\
\hline Esq3 & 20 & 0.00393579 & - & - & 0.01946166 & - & - \\
\hline \multirow[t]{6}{*}{$\epsilon=0$} & 40 & 0.00083461 & 2.237475 & 0.6799 & 0.01007991 & 0.949152 & 0.1731 \\
\hline & 80 & 0.00018260 & 2.192385 & 0.5940 & 0.00511737 & 0.978008 & 0.1887 \\
\hline & 160 & 00004396 & 2.054229 & 0.3568 & 0.00253917 & 1.011041 & 0.2132 \\
\hline & 320 & 0.00001085 & 2.018076 & 0.3045 & 0.00125876 & 1.012356 & 0.2144 \\
\hline & 640 & 0.00000269 & 2.010154 & 0.2925 & 0.00062875 & 1.001445 & 0.2028 \\
\hline & 1280 & 0.00000067 & 2.005005 & 0.2840 & 0.00031306 & 1.006033 & 0.2083 \\
\hline \multirow{7}{*}{$\begin{array}{l}\text { Esq3 } \\
\epsilon=0.1\end{array}$} & 20 & 0.00351657 & - & - & 0.02633534 & - & \\
\hline & 40 & 0.00075924 & 2.211537 & 0.5723 & 0.01337499 & 0.977461 & 0.2500 \\
\hline & 80 & & 2.089 & 0.3969 & 6952 & 1.003882 & 0.2706 \\
\hline & 160 & 358 & 2.033160 & 0.3225 & 2468 & 1.004367 & 0.2711 \\
\hline & 320 & 079 & 2.013653 & 0.2961 & 0.00165975 & 1.002243 & 0.2686 \\
\hline & 640 & 0.00000268 & 2.006081 & 0.2850 & 0.00082992 & 0.999928 & 0.2654 \\
\hline & 1280 & 0.00000067 & 2.002812 & 0.2796 & 0.00041571 & 0.997363 & 0.2615 \\
\hline \multirow{7}{*}{$\begin{array}{l}\text { Esq3 } \\
\epsilon=0.01\end{array}$} & 20 & 0.00371874 & & & 0.02181224 & & \\
\hline & 40 & & 2.221 & 6199 & 002 & 928 & 0.1961 \\
\hline & 80 & & 2.14 & 4942 & 6076 & 0.992140 & 0.2199 \\
\hline & 160 & 04386 & 2.037419 & 0.3307 & 0.00281657 & 1.007055 & 0.2324 \\
\hline & 320 & 0.00001083 & 2.017482 & 0.3030 & 0.00140726 & 1.001048 & 0.2263 \\
\hline & 640 & & 2.008681 & 0.2898 & 112 & 1.005156 & 0.2311 \\
\hline & 1280 & 0.00000067 & 2.004190 & 0.2824 & 0.00035032 & 1.000967 & 0.2256 \\
\hline \multirow{7}{*}{$\begin{array}{l}\text { Esq } 4 \\
\epsilon=0\end{array}$} & 20 & & & & & - & - \\
\hline & 40 & & 2.223 & 6061 & & 0.960106 & 0.2015 \\
\hline & 80 & & 2.109 & 0.4307 & 4690 & 1.007705 & 0.2323 \\
\hline & 160 & 0.00004382 & 2.034970 & 0.3269 & 0.00281106 & 1.006346 & 0.2312 \\
\hline & 320 & & $2.016 \mathrm{~s}$ & 3021 & 281 & 1.002791 & 0.2276 \\
\hline & 640 & & 2.008262 & 890 & 124 & 1.000331 & 0.2248 \\
\hline & 1280 & 0.00000067 & 2.003994 & 0.2820 & 0.00 & 0.999231 & 0.2234 \\
\hline \multirow{7}{*}{$\begin{array}{l}\text { Esq4 } \\
\epsilon=0.1\end{array}$} & 20 & & - & - & 5160 & 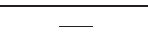 & - \\
\hline & 40 & & 2.217 & 5922 & & .960593 & 0.2087 \\
\hline & 80 & & 2.103833 & 0.4211 & 0.00582933 & 1.010932 & 0.2427 \\
\hline & 160 & 4379 & 2.034790 & 0.3264 & 0.00289905 & 1.007747 & 0.2399 \\
\hline & 320 & 0.00001082 & 2.016508 & 0.3013 & 0.00145093 & 0.998598 & 0.2305 \\
\hline & 640 & 0.00000269 & 2.007998 & 0.2885 & 00072230 & 1.006305 & 0.2396 \\
\hline & 1280 & 00000067 & 2.003864 & 0.2817 & 0.00036120 & 0.999807 & 0.2308 \\
\hline \multirow{7}{*}{$\begin{array}{l}\text { Esq4 } \\
\epsilon=0.01\end{array}$} & 20 & & - & - & 7259 & - & - \\
\hline & 40 & & 2.223000 & 0.6044 & 0.01140038 & 0.959695 & 0.2020 \\
\hline & 80 & & 2.109028 & 0.4295 & 0.00566739 & 1.008326 & 0.2337 \\
\hline & 160 & 382 & 2.034945 & 0.3268 & 0.00282076 & 1.006597 & 0.2322 \\
\hline & 320 & 00001082 & 2.016910 & 0.3020 & 0014 & 1.002403 & 0.2280 \\
\hline & 640 & & 2.0082 & 0.2890 & 3364 & 1.000764 & 0.2261 \\
\hline & 1280 & 0.00000067 & 2.003980 & 0.2820 & 0.00035231 & 0.997978 & 0.2225 \\
\hline
\end{tabular}


Tabela 6.5: Continuação da Tabela 6.4.

\begin{tabular}{|c|c|c|c|c|c|c|c|}
\hline \multirow[t]{2}{*}{ Esquema } & \multirow[t]{2}{*}{$N$} & \multicolumn{3}{|c|}{ Antes do choque } & \multicolumn{3}{|c|}{ Depois do choque } \\
\hline & & Erro & $q$ & $C$ & Erro & $q$ & $C$ \\
\hline Esq5 & 20 & 0.00352576 & - & - & 0.02383964 & - & - \\
\hline \multirow[t]{6}{*}{$\epsilon=0$} & 40 & 0.00076334 & 2.207531 & 0.5685 & 0.01204513 & 0.984912 & 0.2302 \\
\hline & 80 & 0.00018109 & 2.075565 & 0.3829 & 0.00592297 & 1.024056 & 0.2589 \\
\hline & 160 & 0.00004398 & 2.041825 & 0.3380 & 0.00294531 & 1.007900 & 0.2439 \\
\hline & 320 & 0.00001084 & 2.019695 & 0.3068 & 0.00147598 & 0.996747 & 0.2322 \\
\hline & 640 & 0.00000269 & 2.009395 & 0.2912 & 0.00073156 & 1.012611 & 0.2517 \\
\hline & 1280 & 0.00000067 & 2.004552 & 0.2831 & 0.00036710 & 0.994786 & 0.2271 \\
\hline \multirow{7}{*}{$\begin{array}{l}\text { Esq5 } \\
\epsilon=0.1\end{array}$} & 20 & 0.00352348 & - & - & 0.02376092 & - & - \\
\hline & 40 & 0.00076271 & 2.207787 & 0.5685 & 0.01202393 & 0.982682 & 0.2283 \\
\hline & 80 & 0.00018095 & 2.075476 & 0.3824 & 0.00591362 & 1.023794 & 0.2582 \\
\hline & 160 & 0.00004396 & 2.041194 & 0.3370 & 0.0029397 & 1.008366 & 0.2439 \\
\hline & 320 & 0.00001084 & 2.019447 & 0.3064 & 0.00147335 & 0.996574 & 0.2316 \\
\hline & 640 & 0.00000269 & 2.009136 & 0.2903 & 0.00073049 & 1.012158 & 0.2507 \\
\hline & 1280 & 0.00000067 & 2.004371 & 0.2871 & 0.00036659 & 0.994685 & 0.2267 \\
\hline \multirow{7}{*}{$\begin{array}{l}\text { Esq5 } \\
\epsilon=0.01\end{array}$} & 20 & 0.00352553 & - & - & 0.02383145 & - & 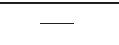 \\
\hline & 40 & 0.00076327 & 2.207560 & 0.5685 & 0.01204295 & 0.984678 & 0.2300 \\
\hline & 80 & 0.00018108 & 2.075551 & 0.3828 & 0.00592200 & 1.024032 & 0.2588 \\
\hline & 160 & 0.00004397 & 2.041761 & 0.3379 & 0.00294473 & 1.007950 & 0.2439 \\
\hline & 320 & 0.00001084 & 2.019672 & 0.3068 & 0.00147570 & 0.996728 & 0.2322 \\
\hline & 640 & 0.00000269 & 2.009382 & 0.2911 & 0.00073145 & 1.012566 & 0.2516 \\
\hline & 1280 & 0.00000067 & 2.004549 & 0.2831 & 0.00036705 & 0.994774 & 0.2271 \\
\hline Esq6 & 20 & 0.00366154 & - & - & 0.02042666 & 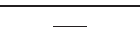 & 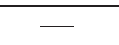 \\
\hline \multirow{6}{*}{$\epsilon=0$} & 40 & 0.00082883 & 2.143299 & 0.5092 & 0.01052053 & 0.957246 & 0.1851 \\
\hline & 80 & 0.00018808 & 2.139686 & 0.5038 & 0.00537703 & 0.968325 & 0.1913 \\
\hline & 160 & 0.00004418 & 2.089708 & 0.4189 & 0.00272116 & 0.982587 & 0.2017 \\
\hline & 320 & 0.00001083 & 2.028129 & 0.3198 & 0.00135770 & 1.003058 & 0.2206 \\
\hline & 640 & 0.00000269 & 2.006155 & 0.2861 & 0.00068340 & 0.990349 & 0.2068 \\
\hline & 1280 & 0.00000067 & 2.005074 & 0.2843 & 0.00034074 & 1.004037 & 0.2238 \\
\hline \multirow{7}{*}{$\begin{array}{l}\text { Esq6 } \\
\epsilon=0.1\end{array}$} & 20 & 0.00354060 & - & - & 0.02214019 & - & - \\
\hline & 40 & 0.00078016 & 2.182138 & 0.5385 & 0.01141913 & 0.955214 & 0.1997 \\
\hline & 80 & 0.00018048 & 2.111905 & 0.4363 & 0.00575452 & 0.988686 & 0.2207 \\
\hline & 160 & 0.00004372 & 2.045247 & 0.3412 & 0.00288791 & 0.994669 & 0.2256 \\
\hline & 320 & 0.00001081 & 2.015054 & 0.2989 & 0.00144362 & 1.000332 & 0.2313 \\
\hline & 640 & 0.00000269 & 2.007303 & 0.2874 & 0.00072173 & 1.000150 & 0.2311 \\
\hline & 1280 & 0.00000067 & 2.003759 & 0.2815 & 0.00036056 & 1.001195 & 0.2325 \\
\hline \multirow{7}{*}{$\begin{array}{l}\text { Esq6 } \\
\epsilon=0.01\end{array}$} & 20 & 0.00363434 & - & - & 0.02067840 & - & - \\
\hline & 40 & 0.00081893 & 2.149876 & 0.5132 & 0.01067733 & 0.953573 & 0.1858 \\
\hline & 80 & 0.00018607 & 2.137839 & 0.4950 & 0.00544911 & 0.970459 & 0.1954 \\
\hline & 160 & 0.00004398 & 2.080772 & 0.4010 & 0.00274145 & 0.991080 & 0.2109 \\
\hline & 320 & 0.00001083 & 2.021551 & 0.3093 & 0.00137210 & 0.998546 & 0.2179 \\
\hline & 640 & 0.00000269 & 2.006974 & 0.2873 & 0.00068823 & 0.995420 & 0.2144 \\
\hline & 1280 & 0.00000067 & 2.004760 & 0.2836 & 0.00034410 & 1.000059 & 0.2203 \\
\hline
\end{tabular}

O cálculo da TV para este problema numa malha de 40 células computacionais é apresentado na Figura 6.14, onde observa-se que a medida em que o tempo avança as TVs dos esquemas da classe FUS-RF decrescem, indicando estabilidade. 
(a) Antes do choque

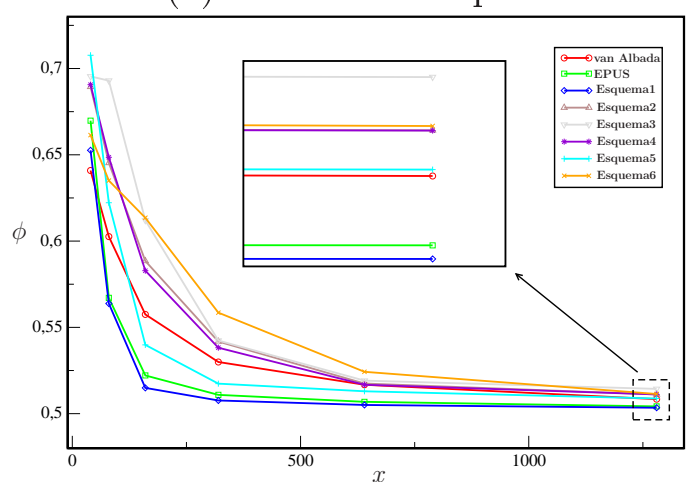

(b) Depois do choque

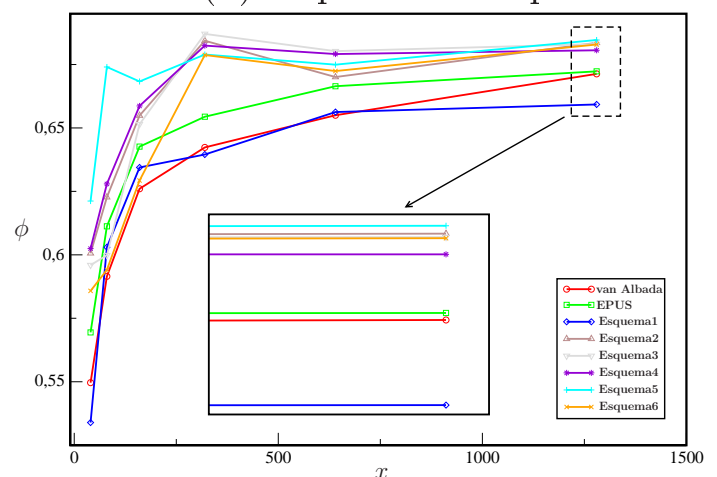

Figura 6.11: Constante de erro $C$ na norma $L_{1}$ para o Teste 3 antes e depois do choque.

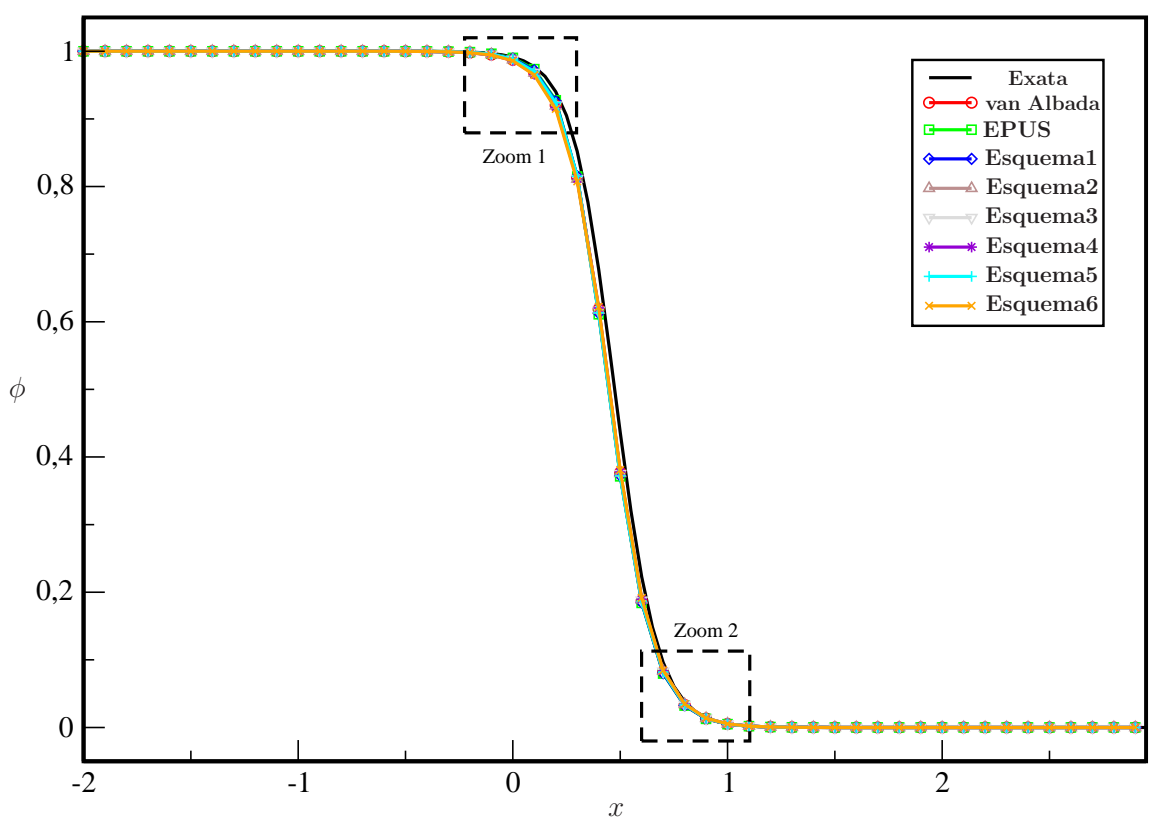

Figura 6.12: Comparação das soluções numéricas e aolução exata para o Teste 4 usando os esquema van Albada, EPUS e Esquemas 1-6 com os melhores parâmetros, mostrando regiões de ampliação.

(a) Zoom 1

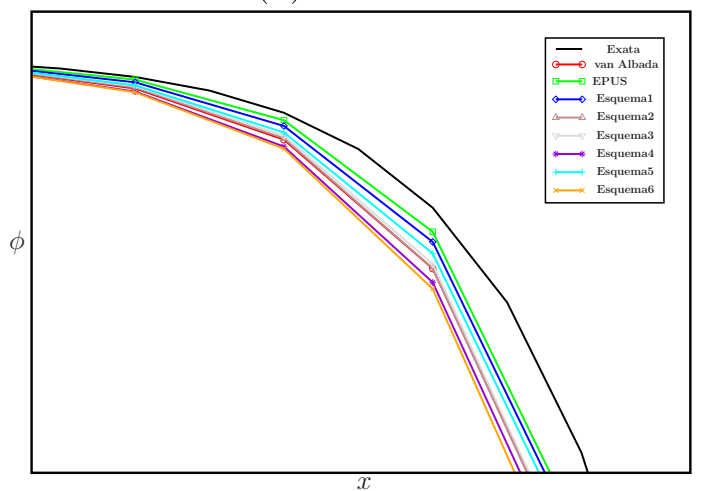

(b) Zoom 2

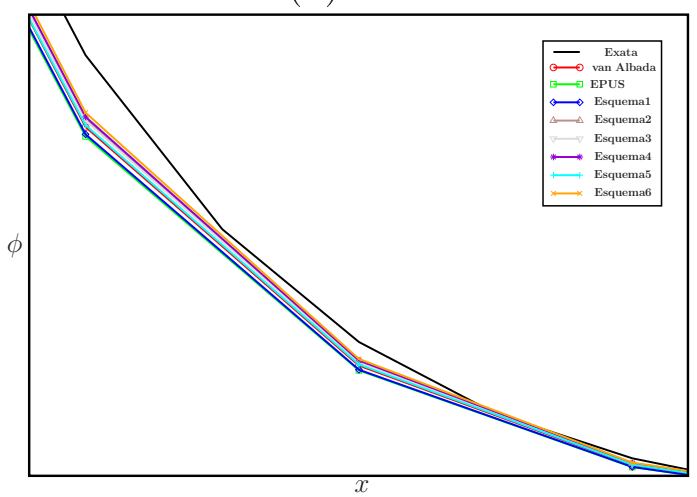

Figura 6.13: Continuação da Figura 6.12. 


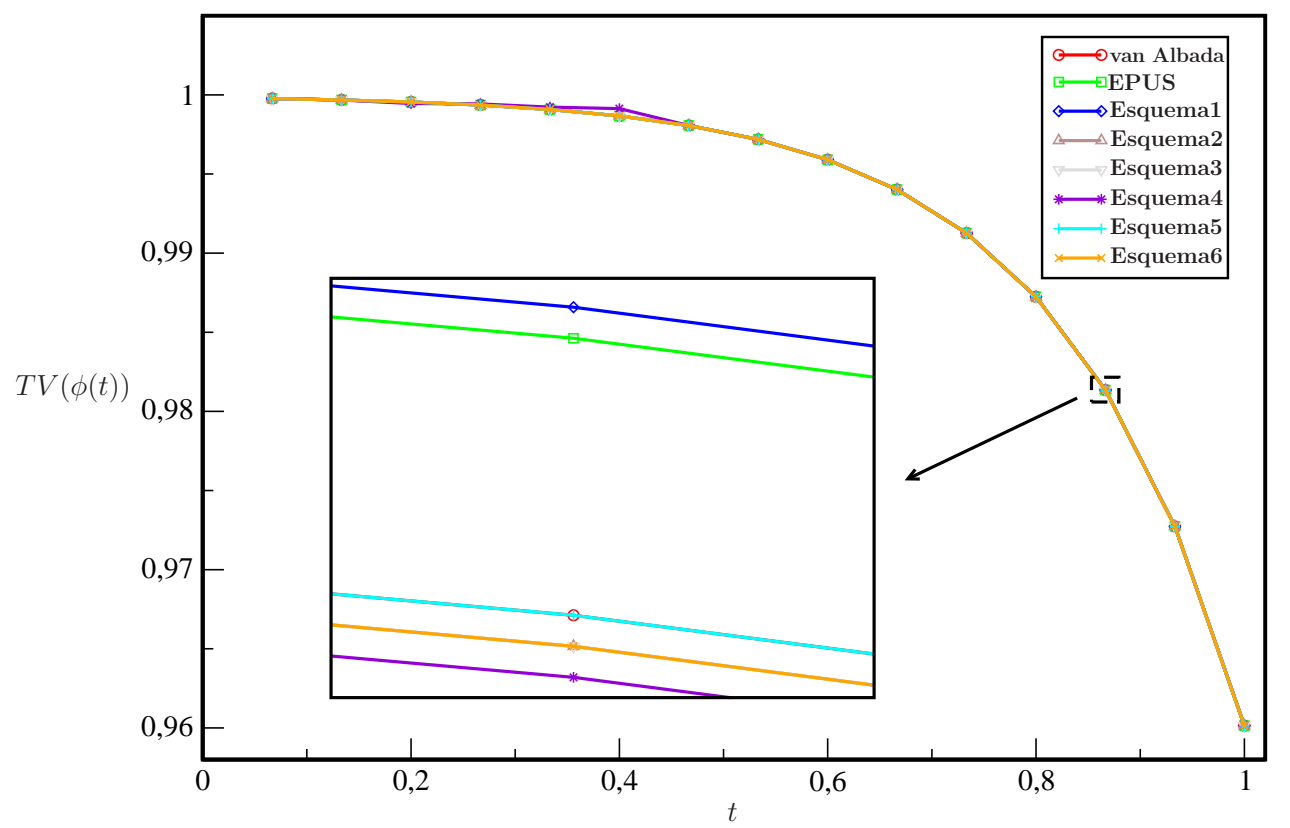

Figura 6.14: Variação total para o Teste 4 com respeito ao tempo.

\subsection{Resultados para equação de águas rasas 1D}

Apresentam-se nesta seção resultados para equações de águas rasas. Em particular, são apresentados resultados de dois testes bem conhecidos (ver LeVeque [21]). O primeiro deles, conhecido como Dam-break, simula o rompimento de uma barreira de contenção e o segundo, o Hydraulic jump, simula um salto abrupto num fluido em repouso.

Teste 5 (Dam-Break): Neste teste um fluido encontra-se em repouso e separado em $x=0$ por uma barreira. A altura $h$ da coluna de fluido é desigual em ambos os lados da barreira. Assume-se que a altura inicial é dada por

$$
h_{0}(x)= \begin{cases}4 & x \leq 0 \\ 1 & x>0\end{cases}
$$

O domínio de cálculo é $[-5,5]$ e é particionado por 150 células computacionais. Para a simulação desse problema, consideram-se $C F L=0.7$ e tempo final de simulação $T=1.2$. A solução de referência utilizada foi obtida com o esquema MC (ver [21]) numa malha de $N=1000$ células computacionais. Foram feitos vários testes com diferentes valores de $\beta$ e de $\epsilon$. Os valores desses parâmetros que forneceram os melhores resultados (isto é, $\beta=0.1545$; $\epsilon=0.01$ para os Esquemas 2 e $3 ; \epsilon=0.1$, para os Esquemas 4 e 6 ; e $\epsilon=0.01$ para o Esquema 5) foram selecionados e, então, mostrados nas Figuras 6.15 e 6.16 para a altura $h$ e suas ampliações, respectivamente, e Figuras 6.17 e 6.18 para a vazão $h u$ e suas ampliações, respectivamente. Dessas figuras, é possível verificar que o Esquema 1 apresenta o melhor 
resultado dentre os esquemas FUS-RF, porém em algumas regiões o esquema EPUS forneceu os melhores resultados.

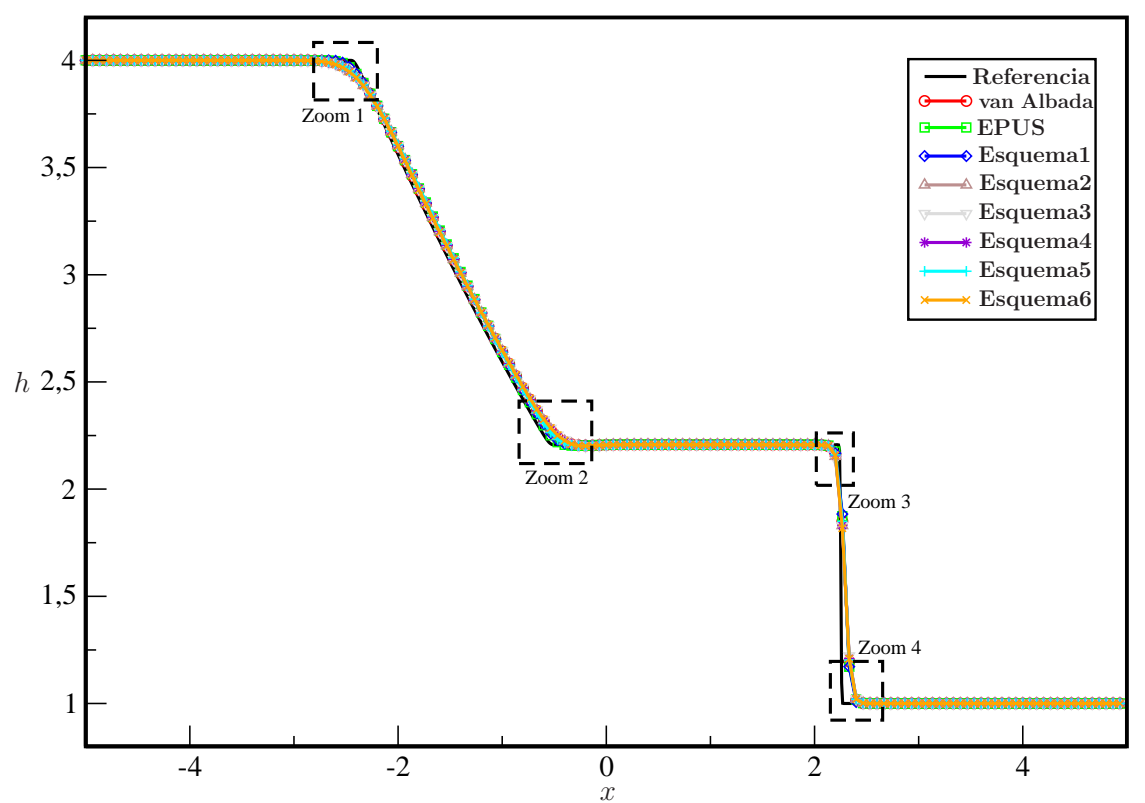

Figura 6.15: Comparação das soluções numéricas e aolução de referência para a altura $h$ do fluido no Teste 5 usando os esquema van Albada, EPUS e Esquemas 1-6 com os melhores parâmetros, mostrando regiões de ampliação.

(a) Zoom 1

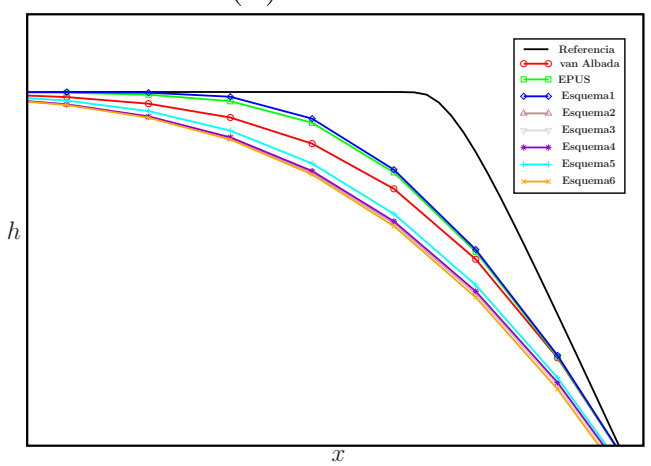

(c) Zoom 3

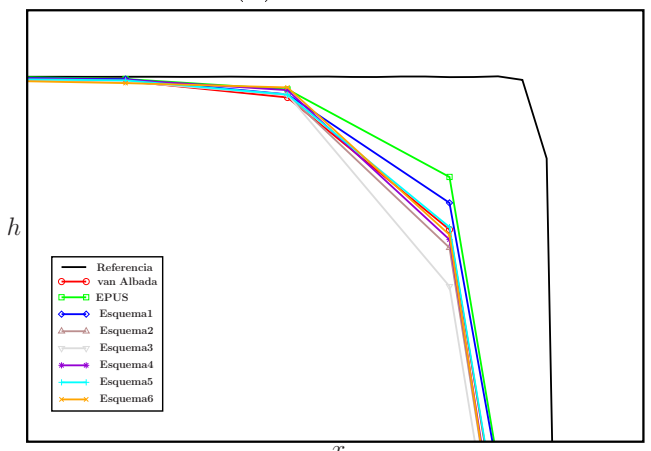

(b) Zoom 2

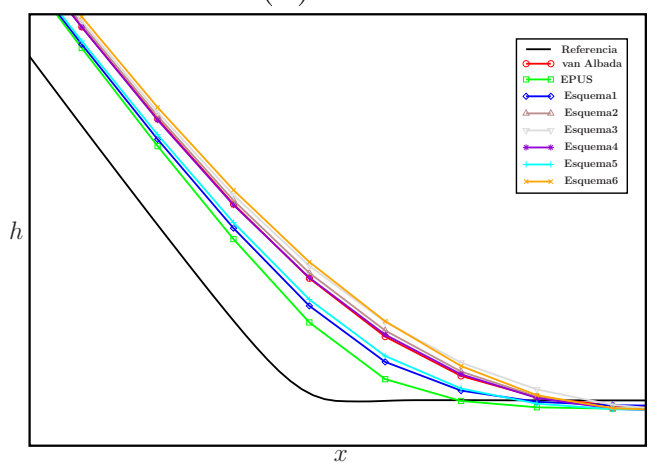

(d) Zoom 4

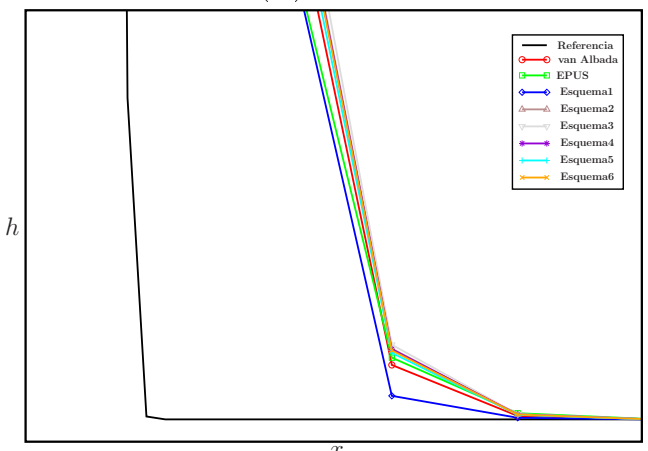

Figura 6.16: Continuação da Figura 6.15. 


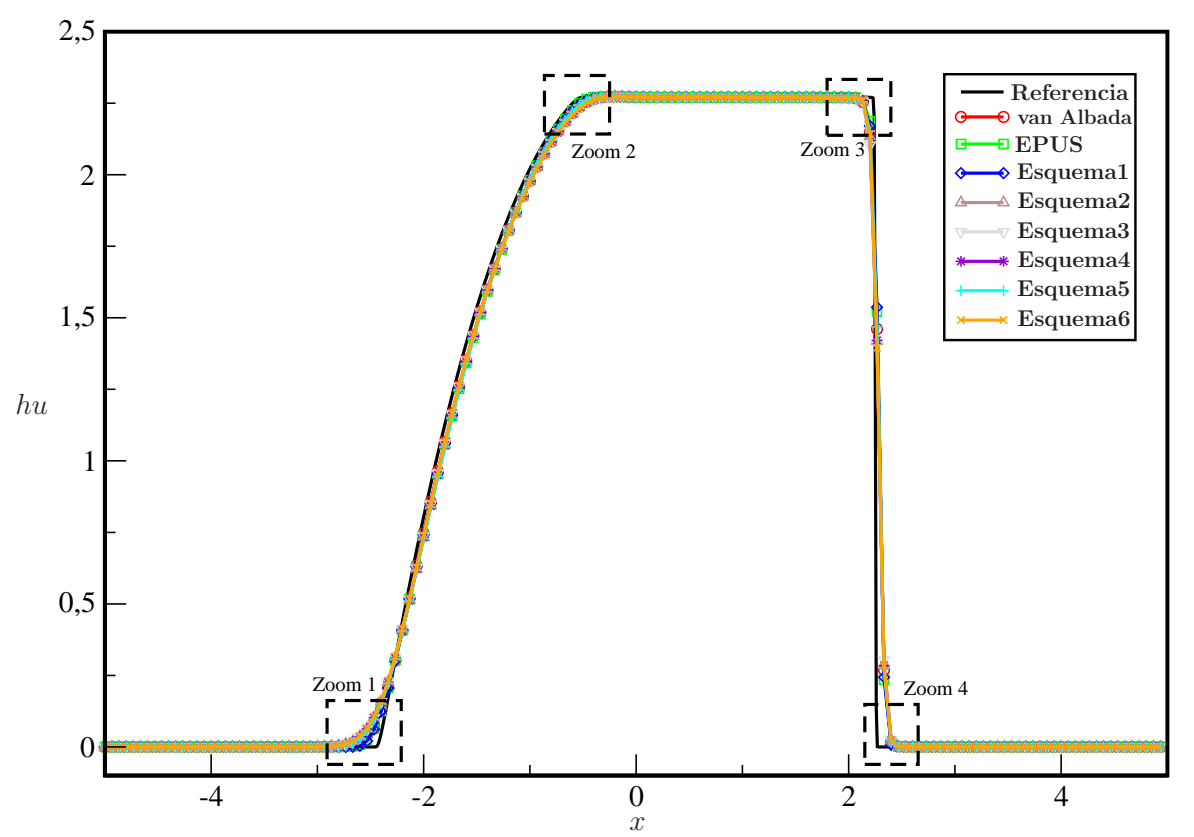

Figura 6.17: Comparação das soluções numéricas e solução de referência para a vazão hu do fluido no Teste 5 usando os esquema van Albada, EPUS e Esquemas 1-6 com os melhores parâmetros, mostrando regiões de ampliação.

(a) Zoom 1

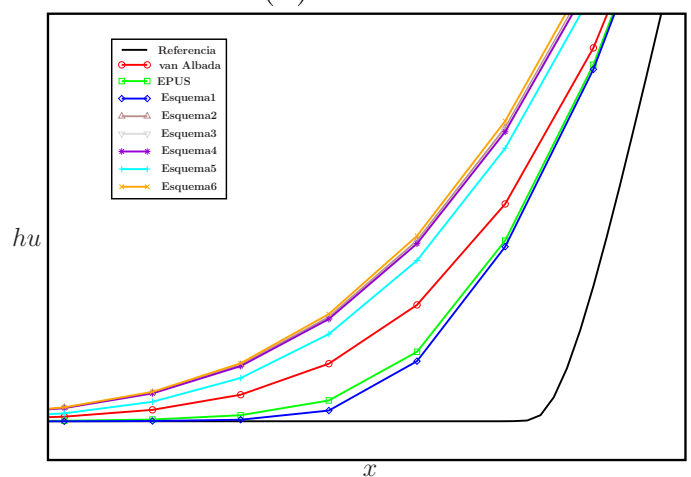

(c) Zoom 3

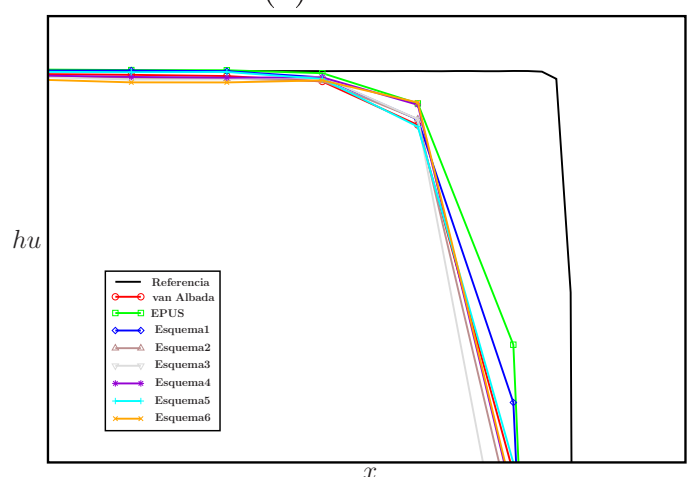

(b) Zoom 2

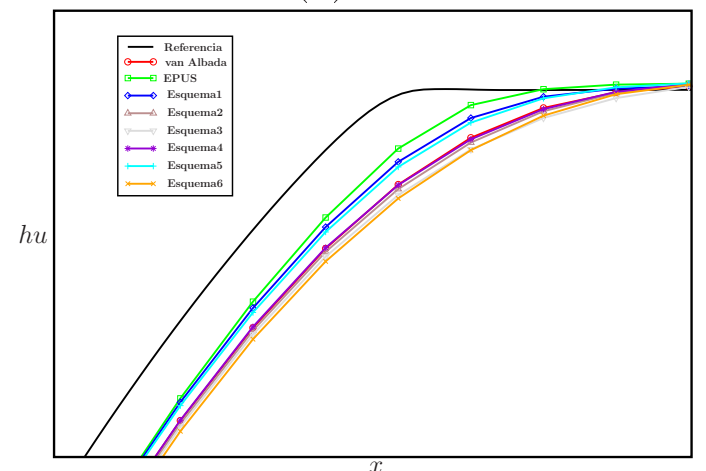

(d) Zoom 4

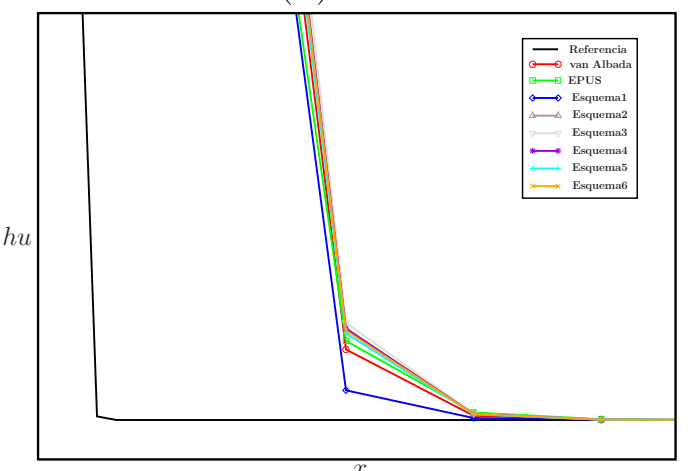

Figura 6.18: Continuação da Figura 6.17. 
As TVs dos métodos numéricos equipados com a família FUS-RF usando-se as malhas $N=10, N=15$ e $N=20$ estão apresentadas na Figura 6.19, onde pode-se observar que, no geral, as TVs decrescem ou permanecem constantes. Em particular, os Esquemas 1, 4, 5 e 6 apresentaram comportamentos semelhantes, enquanto que os Esquemas 2 e 3 diferiram deste comportamento na malha $N=15$.

(a) Esquema $1 \beta=0.1545$

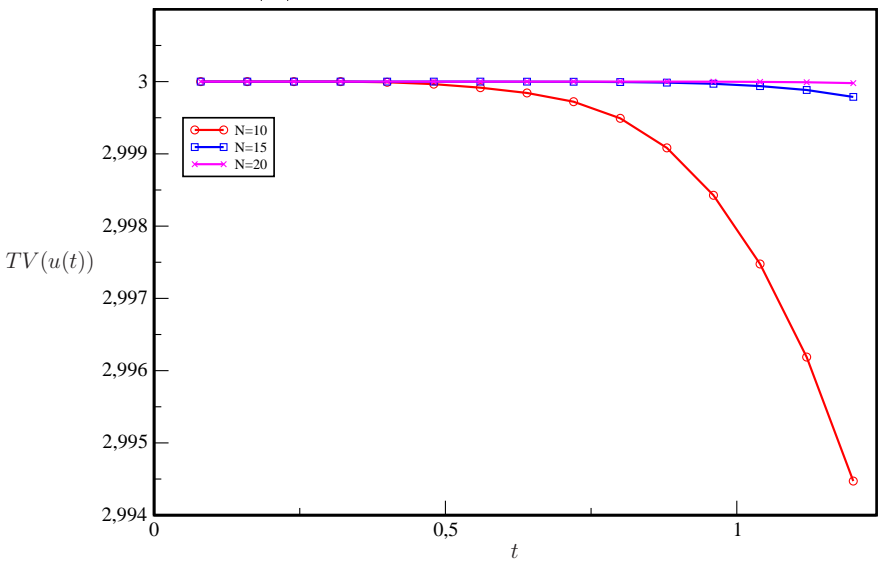

(c) Esquema $3 \epsilon=10^{-} 2$

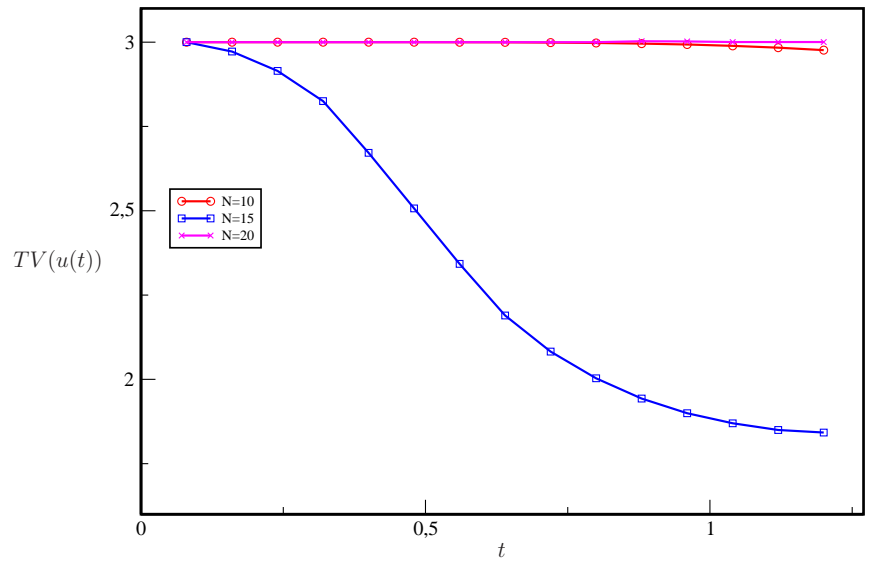

(e) Esquema $5 \epsilon=0$

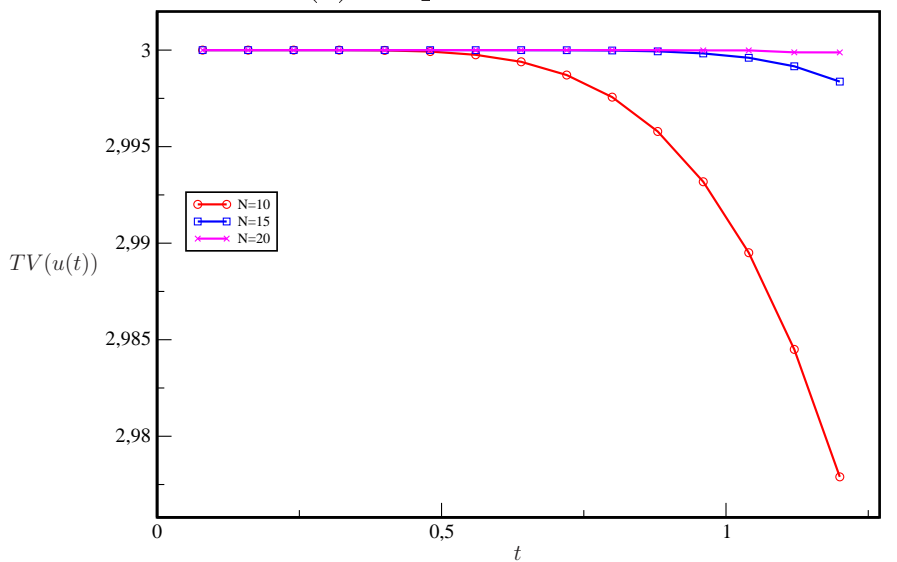

(b) Esquema $2 \epsilon=10^{-} 2$

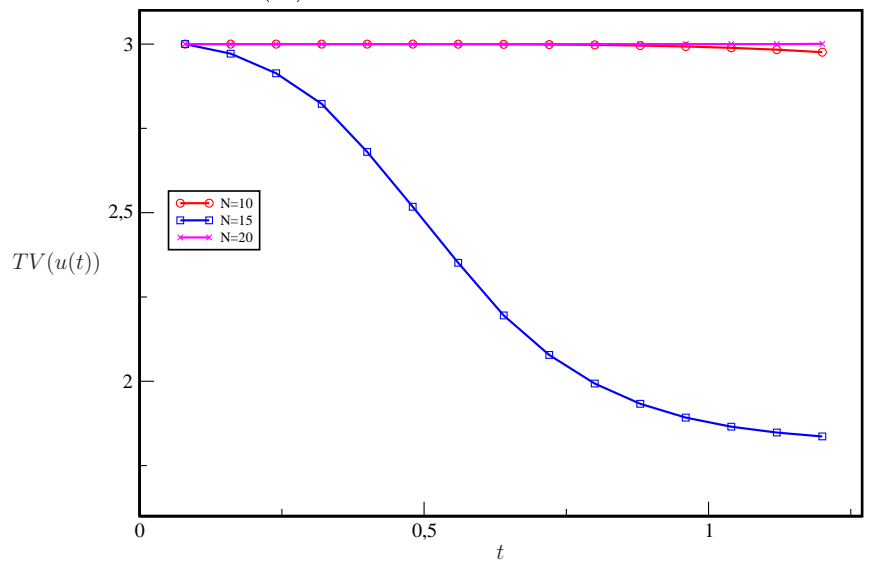

(d) Esquema $4 \epsilon=10^{-} 1$

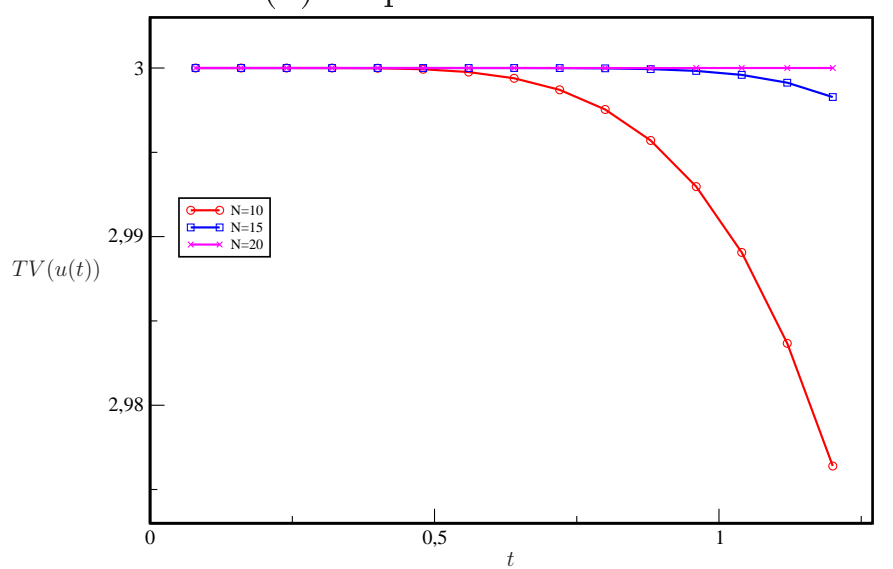

(f)Esquema $6 \epsilon=10^{-} 1$

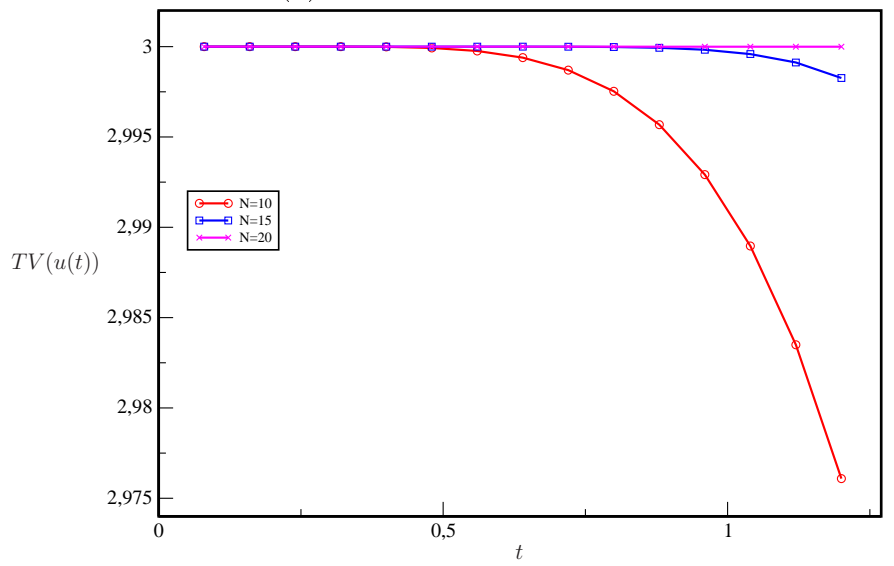

Figura 6.19: Variação total em várias malhas para o Teste 5 com respeito ao tempo. 
Teste 6 (Hydraulic jump): O problema do salto hidráulico é interessante pois parte-se de uma solução (inicialmente) suave, formada por uma única coluna de fluido, que se divide em duas, logo após o rompimento das condições de repouso. Este teste pode ser encontrado em LeVeque [21]. As condições iniciais são dadas por

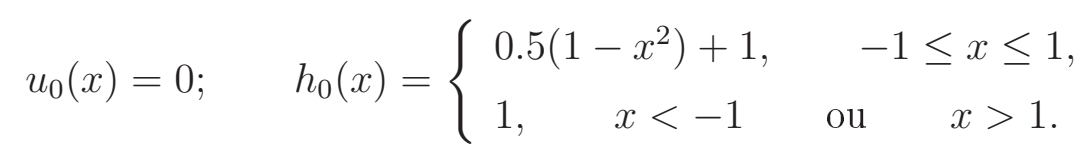

Para a simulação, consideram-se uma malha de 200 células no domínio $[-5,5]$, o número de Courant $\theta=0.9$ e tempo final $T=2.5$. A solução de referência foi calculada usando o esquema MC numa malha de 1000 células. Os resultados numéricos encontrados nas Figuras 6.20 e 6.21, para a vazão $h u$ e ampliações e Figuras 6.22 e 6.23, para a altura $h$ e ampliações mostram que o Esquema 1 com $\beta=0.1545$ foi, de maneira geral, o que melhor aproximou a solução de referência, porém em algumas regiões o esquema EPUS comportou-se melhor. O Esquema 5 com $\epsilon=0$ também apresentou bom resultado, mostrando-se melhor que o van Albada e, em algumas regiões, aproximando-se dos Esquemas 1 e EPUS.

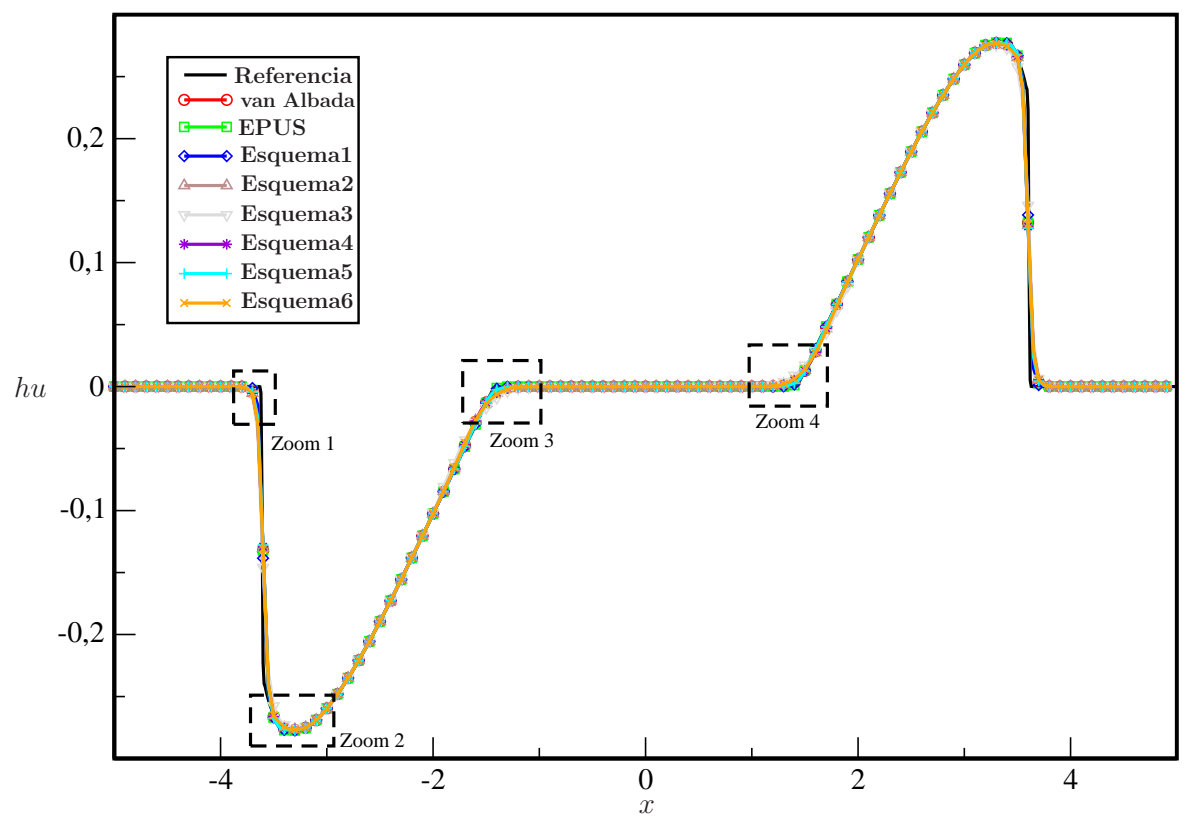

Figura 6.20: Comparação das soluções numéricas e solução de referência para a vazão hu do fluido no Teste 6 usando os esquema van Albada, EPUS e Esquemas 1-6 com os melhores parâmetros, mostrando regiões de ampliação.

Para esse problema, o erro e a ordem observada dos métodos numéricos foram calculados e os resultados encontram-se na Tabela 6.6. Vê-se que, dentre os esquemas estudados, o esquema EPUS forneceu a melhor ordem de convergência. Os Esquemas 1 e 5 proporcionaram bons resultados, com ordem de convergência superior à do esquema van Albada. Os demais esquemas, apesar de não superarem a ordem de convergência dos esquemas de comparação (o convencional) van Albada e (o atual) EPUS, forneceram resultados satisfatórios, uma vez que o erro decresce nas três normas ao se refinar a malha. 
(a) Zoom 1

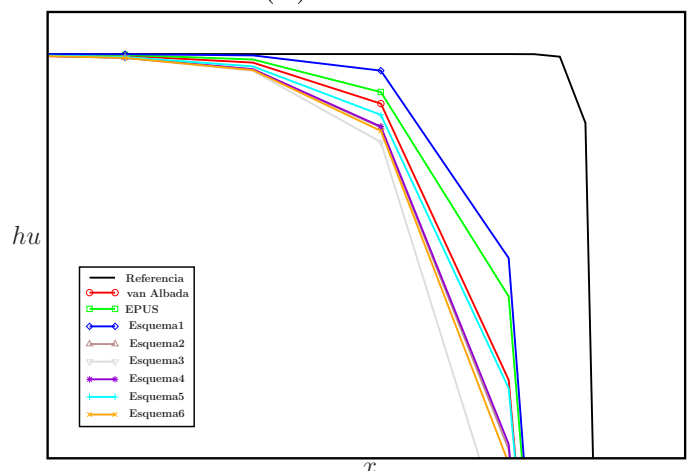

(a) Zoom 3

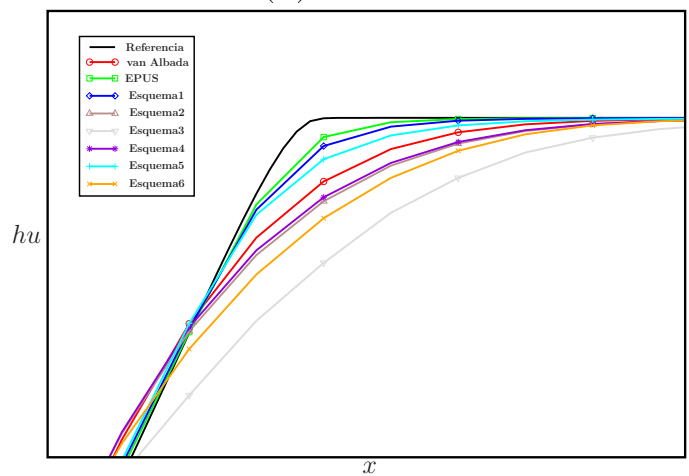

(b) Zoom 2

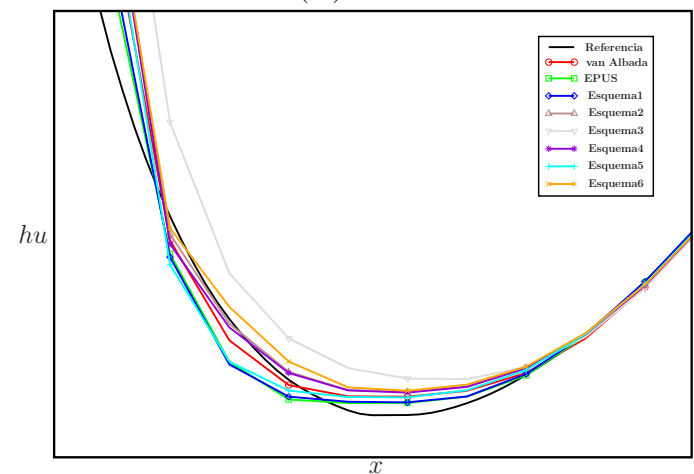

(b) Zoom 4

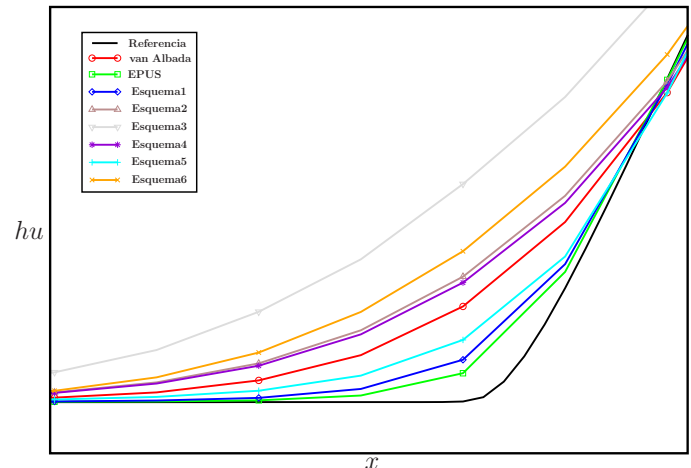

Figura 6.21: Continuação da Figura 6.20.

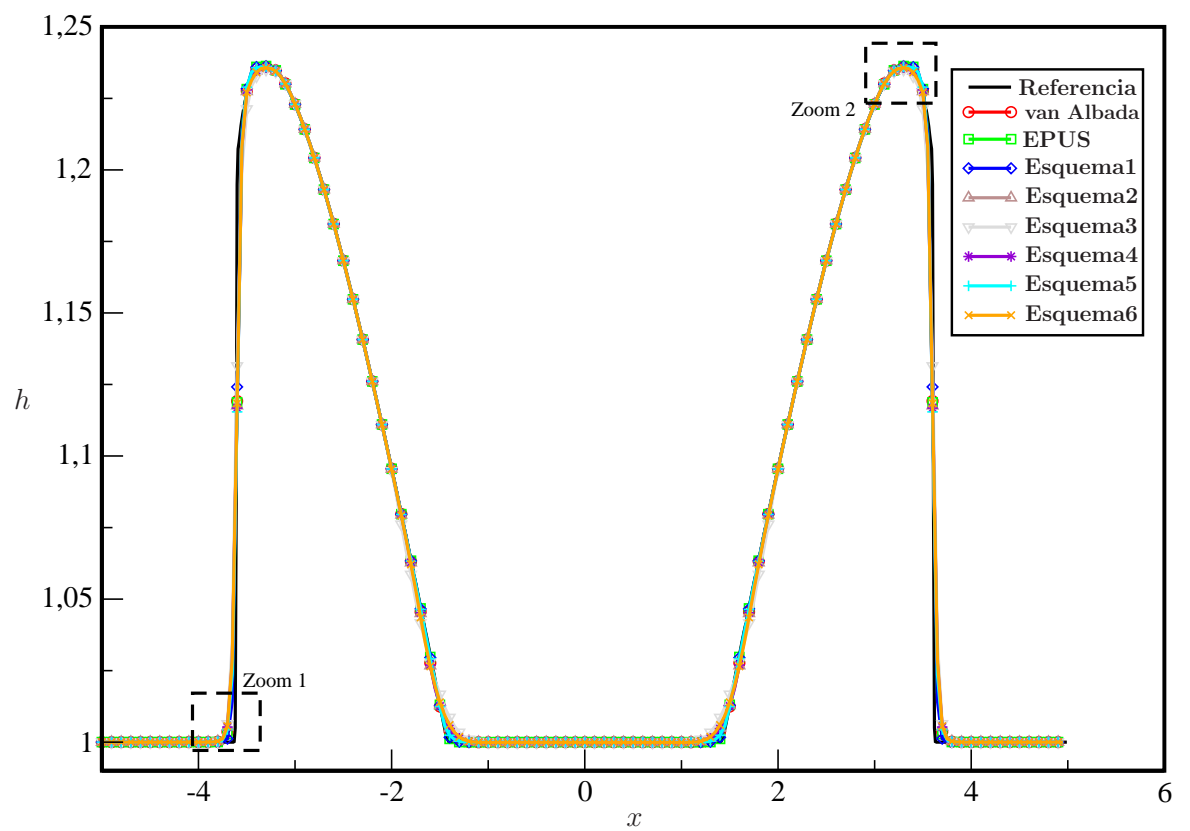

Figura 6.22: Comparação das soluções numéricas e solução de referência para a altura $h$ do fluido no Teste 6 usando os esquema van Albada, EPUS e Esquemas 1-6 com os melhores parâmetros, mostrando regiões de ampliação. 
(a) Zoom 1

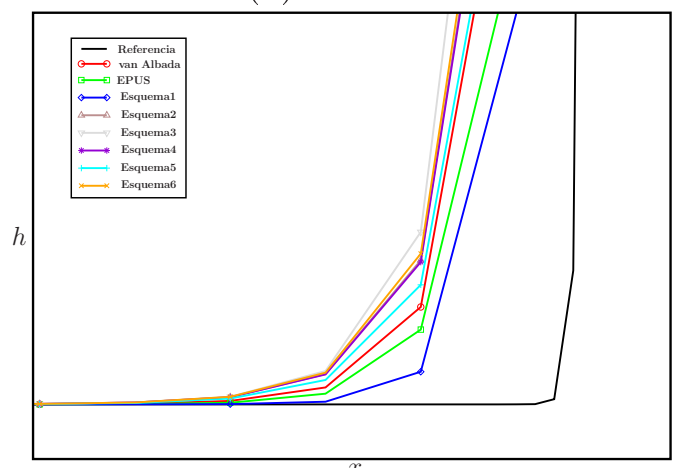

(b) Zoom 2

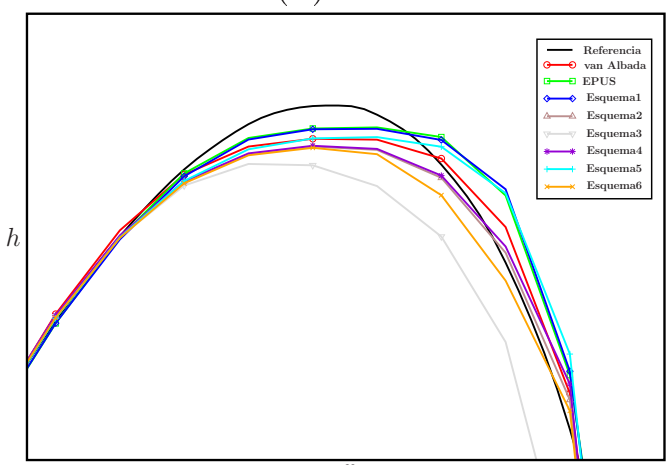

Figura 6.23: Continuação da Figura 6.22.

Tabela 6.6: Comparação dos erros e ordens observadas nas normas $L_{1}, L_{2}$ e $L_{\infty}$ para o Teste 6, usando os esquemas van Albada, EPUS e os Esquemas 1-6 com os melhores parâmetros.

\begin{tabular}{|c|c|c|c|c|c|c|c|}
\hline & \multirow[t]{2}{*}{$N$} & \multicolumn{2}{|c|}{$L_{1}$} & \multicolumn{2}{|c|}{$L_{2}$} & \multicolumn{2}{|c|}{$L_{\infty}$} \\
\hline & & Erro & $q$ & Erro & $q$ & Erro & $q$ \\
\hline \multirow[t]{4}{*}{ Van Albada } & 125 & 0.00187709 & - & 0.00005044 & - & 0.03887380 & - \\
\hline & 250 & 0.00102684 & 0.870291 & 0.00004779 & 0.077784 & 0.06379831 & 0.714720 \\
\hline & 500 & 0.00041999 & 1.289774 & 0.00001343 & 1.830396 & 0.04080667 & 0.644713 \\
\hline & 1000 & 0.00011136 & 1.915073 & 0.00000117 & 3.517214 & 0.01382453 & 1.561574 \\
\hline \multirow[t]{4}{*}{ EPUS } & 125 & 0.00135873 & - & 0.00003265 & - & 0.03143030 & - \\
\hline & 250 & 0.00089944 & 0.595164 & 0.00005054 & 0.630134 & 0.06707364 & 1.093590 \\
\hline & 500 & 0.00034964 & 1.363128 & 0.00001133 & 2.156197 & 0.03949987 & 0.763898 \\
\hline & 1000 & 0.00007755 & 2.172586 & 0.00000068 & 4.058924 & 0.01056764 & 1.902195 \\
\hline Esq 1 & 125 & 0.00127702 & - & 0.00002965 & - & 0.03168761 & - \\
\hline \multirow[t]{3}{*}{$\beta=0.1545$} & 250 & 0.00078893 & 0.694812 & 0.00004048 & 0.449421 & 0.06040755 & 0.930810 \\
\hline & 500 & 0.00031074 & 1.344171 & 0.00000916 & 2.144091 & 0.03482583 & 0.794571 \\
\hline & 1000 & 0.00007713 & 2.010321 & 0.00000077 & 3.570110 & 0.01195770 & 1.542218 \\
\hline \multirow{4}{*}{$\begin{array}{l}\text { Esq } 2 \\
\epsilon=0.01\end{array}$} & 125 & 0.00226587 & - & 0.00006606 & - & 0.04354266 & - \\
\hline & 250 & 0.00119134 & 0.927484 & 0.00005209 & 0.342872 & 0.06521020 & 0.582668 \\
\hline & 500 & 0.00050066 & 1.250664 & 0.00001642 & 1.665389 & 0.04459313 & 0.548276 \\
\hline & 1000 & 0.00013701 & 1.869534 & 0.00000155 & 3.402949 & 0.01671548 & 1.415637 \\
\hline \multirow{4}{*}{$\begin{array}{l}\text { Esq 3 } \\
\epsilon=0\end{array}$} & 125 & 0.00324340 & - & 0.00009053 & - & 0.05342830 & - \\
\hline & 250 & 0.00159348 & 1.025327 & 0.00004424 & 1.032975 & 0.05309097 & 0.009138 \\
\hline & 500 & 0.00072778 & 1.130593 & 0.00001876 & 1.237698 & 0.04226270 & 0.329082 \\
\hline & 1000 & 0.00026851 & 1.438505 & 0.00000375 & 2.320025 & 0.02391483 & 0.821479 \\
\hline \multirow{4}{*}{$\begin{array}{l}\text { Esq } 4 \\
\epsilon=0.1\end{array}$} & 125 & 0.00221489 & - & 0.00006520 & - & 0.04306088 & - \\
\hline & 250 & 0.00118054 & 0.907789 & 0.00005274 & 0.305946 & 0.06583369 & 0.612448 \\
\hline & 500 & 0.00049177 & 1.263392 & 0.00001641 & 1.683827 & 0.04494048 & 0.550810 \\
\hline & 1000 & 0.00013251 & 1.891852 & 0.00000149 & 3.459410 & 0.01657911 & 1.438649 \\
\hline \multirow{4}{*}{$\begin{array}{l}\text { Esq } 5 \\
\epsilon=0\end{array}$} & 125 & 0.00176175 & - & 0.00005037 & - & 0.03807707 & - \\
\hline & 250 & 0.00102947 & 0.775107 & 0.00005499 & 0.126409 & 0.06876466 & 0.852745 \\
\hline & 500 & 0.00041406 & 1.313964 & 0.00001477 & 1.895646 & 0.04372010 & 0.653371 \\
\hline & 1000 & 0.00010296 & 2.007706 & 0.00000121 & 3.602187 & 0.01492261 & 1.550796 \\
\hline \multirow{4}{*}{$\begin{array}{l}\text { Esq } 6 \\
\epsilon=0.1\end{array}$} & 125 & 0.00243438 & - & 0.00007078 & - & 0.04477129 & - \\
\hline & 250 & 0.00126548 & 0.943873 & 0.00005176 & 0.451520 & 0.06450636 & 0.526867 \\
\hline & 500 & 0.00053945 & 1.230120 & 0.00001661 & 1.639351 & 0.04417909 & 0.546078 \\
\hline & 1000 & 0.00015668 & 1.783618 & 0.00000156 & 3.407091 & 0.01668743 & 1.404602 \\
\hline
\end{tabular}




\subsection{Resultados para as equações de Euler 1D}

As equações de Euler são uma simplificação realista das equações completas de Navier-Stokes, pois elas são capazes de simular efeitos de viscosidade no fluido ou de condução de calor. Com este sistema de equações foram realizados três testes numéricos, à saber: um problema com condição inicial suave, e dois outros envolvendo descontinuidades na solução.

Teste 7 (Hump): As condições iniciais neste problema são suaves e definidas para cada ponto $i$, de uma malha de tamanho $N=400$, como

$$
\begin{gathered}
{\left[\rho_{0}, \quad u_{0}, \quad E_{0}\right]^{T}=\left[\begin{array}{ll}
\rho_{0}(i), \quad u_{0}(i), \quad E_{0}(i)
\end{array}\right]^{T}=} \\
=\left[1+0.5 \exp \left[80-\left(-1.5375+7.510^{-3} i\right)^{2}\right], 0, \frac{10}{4}\left(1+0.5 \exp \left[80-\left(-1.5375+7.510^{-3} i\right)^{2}\right]\right)\right]^{T},
\end{gathered}
$$

Foram utilizados na simulação desse problema tempo final $T=0.6$, número de Courant $\theta=0.7$ e domínio $[-1.5,1.5]$. Na Figura 6.24 pode-se verificar o perfil da pressão $p$ ao longo do domínio.

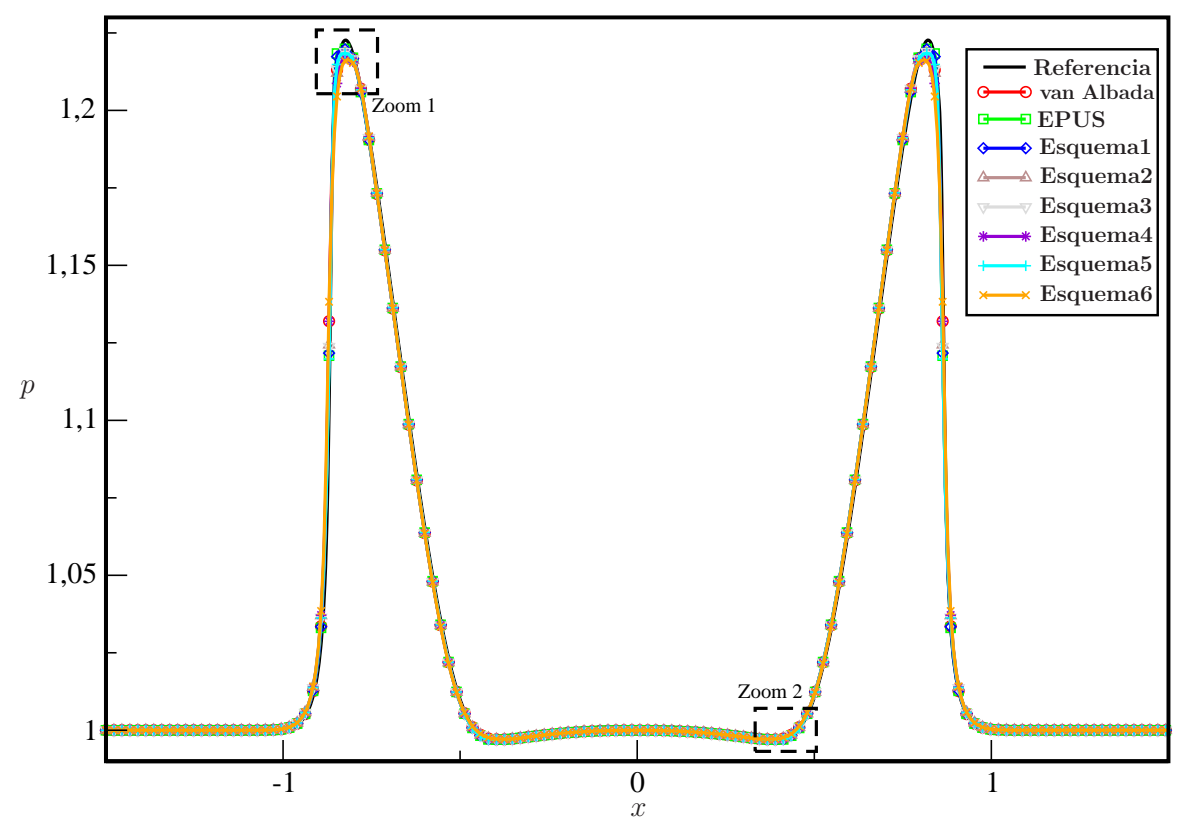

Figura 6.24: Comparação das soluções numéricas e a solução de referência para a pressão $p$ no Teste 7, usando os esquema van Albada, EPUS e Esquemas 1-6 com os melhores parâmetros, mostrando regiões de ampliação.

Na região de Zoom 1 (Figura 6.25 (a)) nota-se que o Esquema 1 e o esquema EPUS forneceram os melhores resultados quando comparados com a solução de referência de LeVeque [21]. As Figuras 6.26- 6.29 apresentam, respectivamente, as soluções para a densidade $\rho$ e a velocidade $u$ e regiões de ampliação, nas quais é possível evidenciar novamente que os Esquema 1 e EPUS produziram, de maneira geral, os melhores resultados. 
(a) Zoom 1

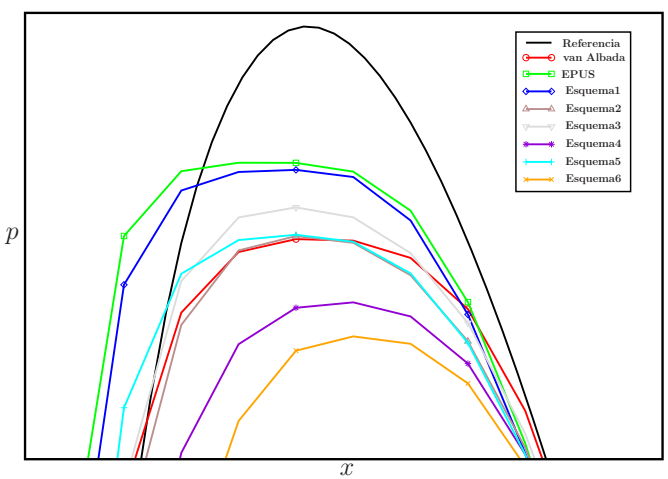

(b) Zoom 2

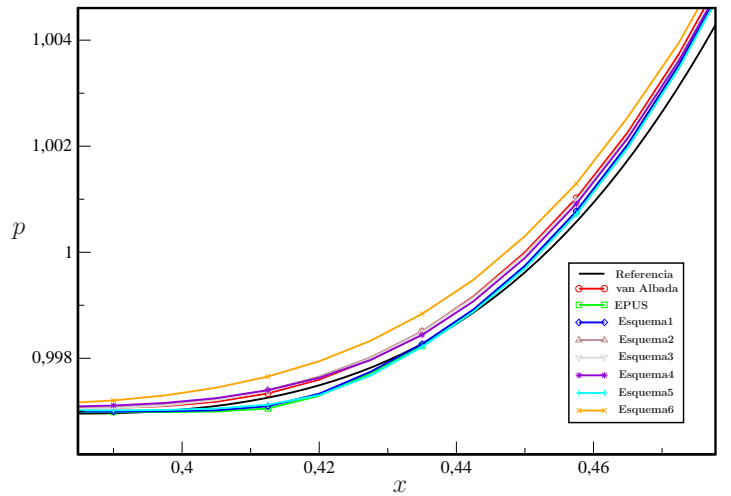

Figura 6.25: Continuação da Figura 6.24.

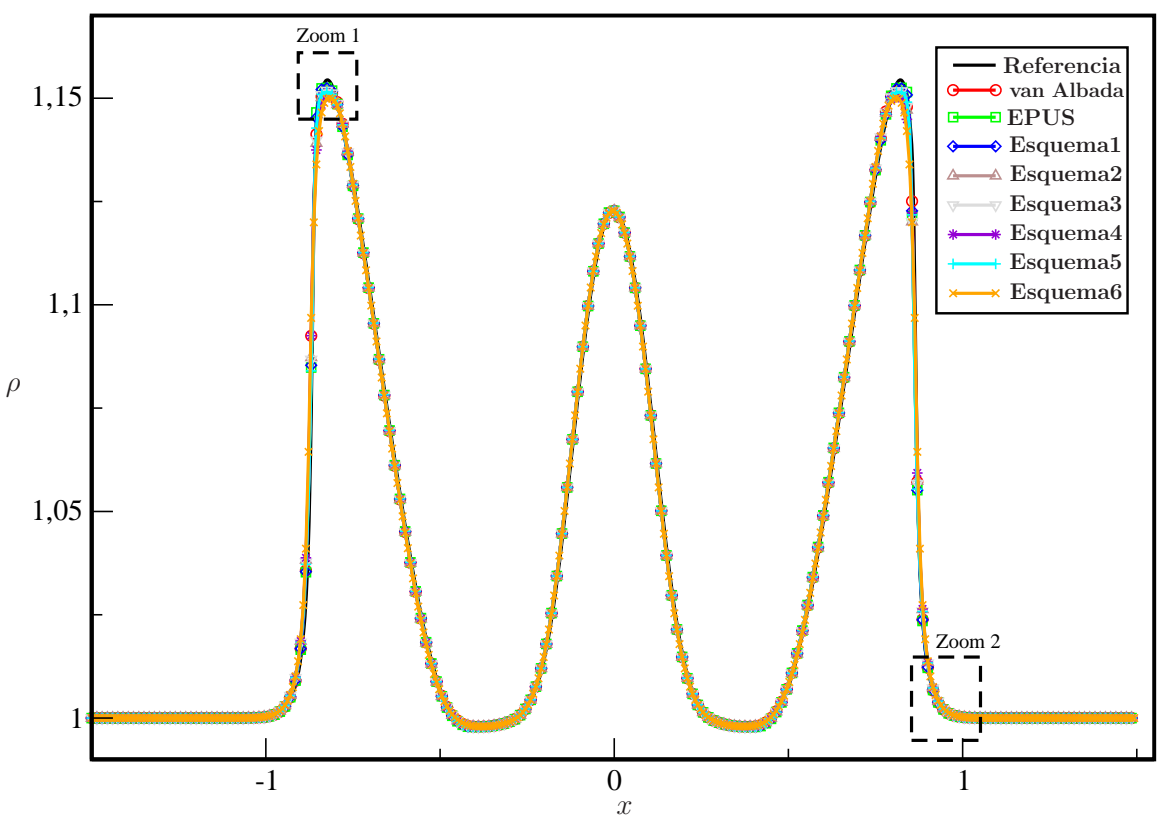

Figura 6.26: Comparação das soluções numéricas e a solução de referência para a densidade $\rho$ no Teste 7, usando os esquema van Albada, EPUS e Esquemas 1-6 com os melhores parâmetros, mostrando regiões de ampliação.

(a) Zoom 1

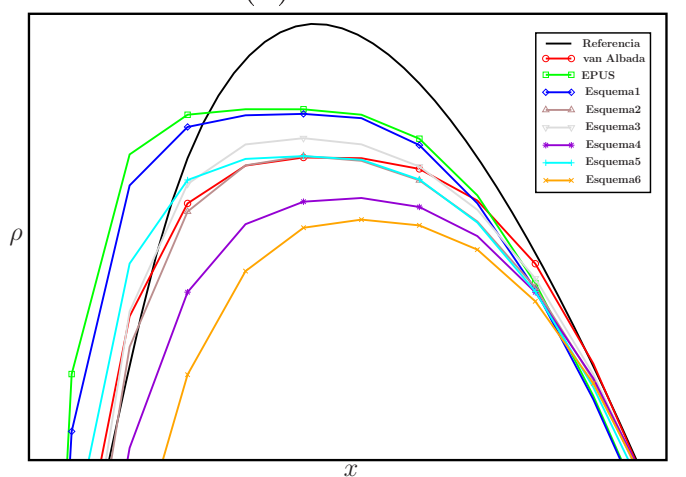

(b) Zoom 2

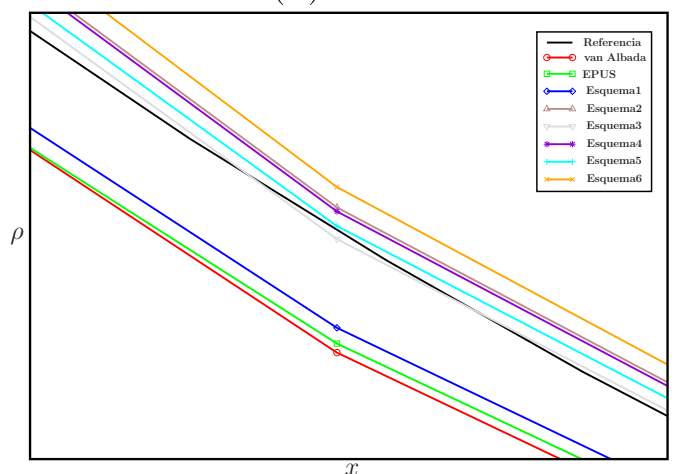

Figura 6.27: Continuação da Figura 6.26. 


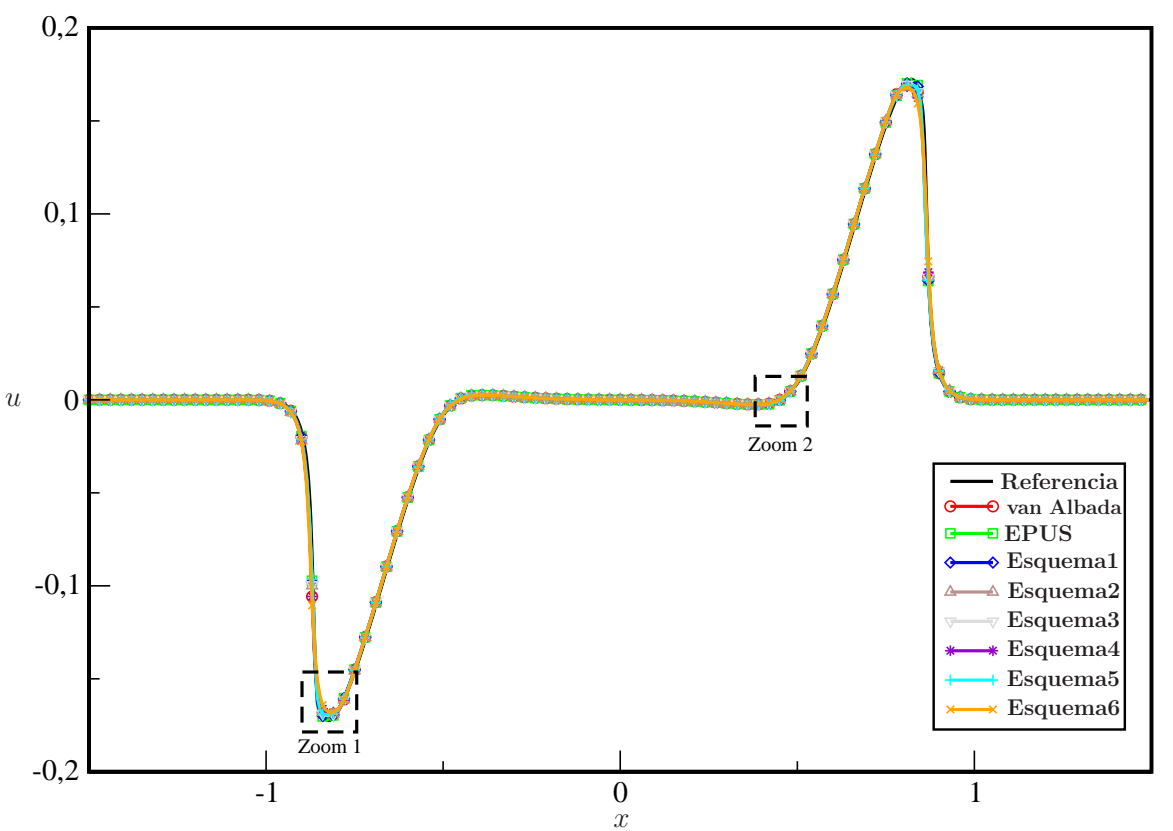

Figura 6.28: Comparação das soluções numéricas e a solução de referência para a velocidade $u$ no Teste 7, usando os esquema van Albada, EPUS e Esquemas 1-6 com os melhores parâmetros, mostrando regiões de ampliação.

(a) Zoom 1

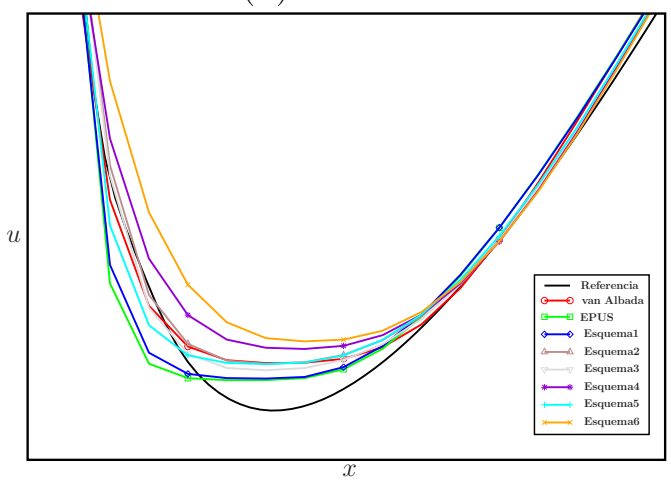

(b) Zoom 2

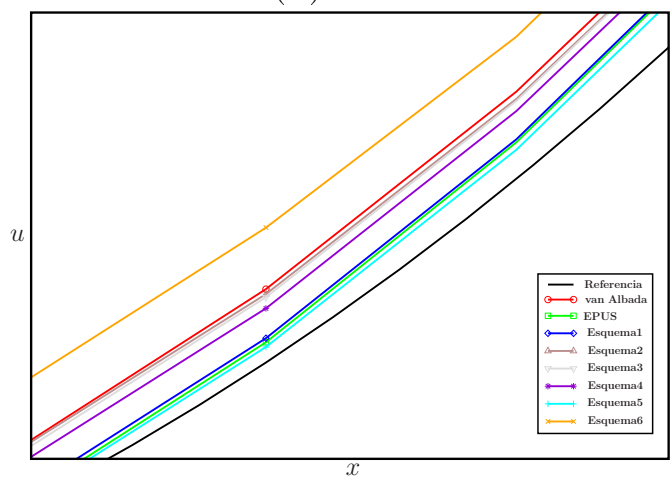

Figura 6.29: Continuação da Figura 6.28.

Nas Tabelas 6.7 e 6.8 encontram-se os dados para o erro e ordem de convergência para todos os esquemas. O Esquema 1 foi testado para três parâmetros $\beta(\beta=0.1545, \beta=0.1605$ e $\beta=0.1670)$ e os demais esquemas para três valores de $\epsilon(\epsilon=0, \epsilon=0.1$ e $\epsilon=0.01)$. Os novos esquemas propostos e os de comparação (van Albada e EPUS) atingiram terceira ordem de precisão na norma $L_{2}$ para ao menos um valor dos parâmetros, com exceção do Esquema 5 que atingiu apenas ordem próxima de 3 (2.894476).

Teste 8 (Tubo de choque de Sod): Esse problema é caracterizado por apresentar condições inicias (à direita e à esquerda de um ponto $x_{0}$ ) distintas para pressão $p$ e densidade $\rho$. Também, nesse problema, as definições $p_{l}$ e $\rho_{l}$ para $x<x_{0}$ e $p_{r}$ e $\rho_{r}$ para $x>x_{0}$ devem satisfazer $p_{l}>p_{r}$ 
Tabela 6.7: Comparação dos erros e ordens observadas nas normas $L_{1}, L_{2}$ e $L_{\infty}$ para o Teste 7, usando os esquemas van Albada, EPUS e os Esquemas 1-6 com vários parâmetros.

\begin{tabular}{|c|c|c|c|c|c|c|c|}
\hline & $N$ & $L$ & & $L$ & & $L_{\mathrm{c}}$ & \\
\hline & & Erro & $q$ & Erro & $q$ & Erro & $q$ \\
\hline Van & 125 & 0.00375119 & - & 0.00006214 & - & 0.04508877 & - \\
\hline Albada & 250 & 0.00158573 & 1.242198 & 0.00001240 & 2.324814 & 0.02871575 & 0.650926 \\
\hline & 500 & 0.00065942 & 1.265879 & 0.00000225 & 2.461425 & 0.01328186 & 1.112385 \\
\hline & 1000 & 0.00021970 & 1.585621 & 0.00000028 & 2.999946 & 0.00659800 & 1.009355 \\
\hline EPUS & 125 & 0.00347746 & - & 0.00005621 & - & 0.04751104 & - \\
\hline & 250 & 0.00153668 & 1.178213 & 0.00001116 & 2.332474 & 0.02428075 & 0.968450 \\
\hline & 500 & 0.00067252 & 1.192159 & 0.00000289 & 1.948372 & 0.01768146 & 0.457576 \\
\hline & 1000 & 0.00022723 & 1.565386 & 0.00000033 & 3.109058 & 0.00737427 & 1.261665 \\
\hline Esq 1 & 125 & 0.00351587 & - & 0.00005851 & - & 0.04929797 & - \\
\hline$\beta=0.1545$ & 250 & 0.00154121 & 1.189812 & 0.00001130 & 2.372043 & 0.02546655 & 0.952925 \\
\hline & 500 & 0.00066390 & 1.215024 & 0.00000276 & 2.030958 & 0.01729562 & 0.558196 \\
\hline & 1000 & 0.00022378 & 1.568832 & 0.00000031 & 3.120848 & 0.00706830 & 1.290971 \\
\hline Esq 1 & 125 & 0.00355292 & - & 0.00005917 & - & 0.04776447 & - \\
\hline$\beta=0.1605$ & 250 & 0.00154980 & 1.196917 & 0.00001147 & 2.365911 & 0.02515376 & 0.925164 \\
\hline & 500 & 0.00066410 & 1.222606 & 0.00000273 & 2.069468 & 0.01747286 & 0.525659 \\
\hline & 1000 & 0.00022288 & 1.575111 & 0.00000031 & 3.137808 & 0.00688448 & 1.343696 \\
\hline Esq 1 & 125 & 0.00361388 & - & 0.00006020 & - & 0.04642497 & - \\
\hline$\beta=0.1670$ & 250 & 0.00156040 & 1.211632 & 0.00001171 & 2.361024 & 0.02495336 & 0.895667 \\
\hline & 500 & 0.00066425 & 1.232106 & 0.00000270 & 2.116012 & 0.01757560 & 0.505660 \\
\hline & 1000 & 0.00022257 & 1.577437 & 0.00000030 & 3.148857 & 0.00668948 & 1.393607 \\
\hline Esq 2 & 125 & 0.00418938 & - & 0.00007242 & - & 0.04291203 & - \\
\hline$\epsilon=0$ & 250 & 0.00180235 & 1.216851 & 0.00001586 & 2.190576 & 0.03021389 & 0.506170 \\
\hline & 500 & 0.00069706 & 1.370520 & 0.00000269 & 2.557773 & 0.01454938 & 1.054254 \\
\hline & 1000 & 0.00022820 & 1.610968 & 0.00000029 & 3.210068 & 0.00473397 & 1.619834 \\
\hline Esq 2 & 125 & 0.00381970 & - & 0.00006354 & - & 0.04631684 & - \\
\hline$\epsilon=0.1$ & 250 & 0.00163907 & 1.220575 & 0.00001373 & 2.210203 & 0.02437632 & 0.926057 \\
\hline & 500 & 0.00066489 & 1.301688 & 0.00000254 & 2.434400 & 0.01498000 & 0.702442 \\
\hline & 1000 & 0.00022230 & 1.580616 & 0.00000027 & 3.190539 & 0.00459987 & 1.703371 \\
\hline Esq 2 & 125 & 0.00411968 & - & 0.00007022 & - & 0.04167598 & - \\
\hline$\epsilon=0.01$ & 250 & 0.00177379 & 1.215695 & 0.00001527 & 2.200343 & 0.02888773 & 0.528759 \\
\hline & 500 & 0.00068980 & 1.362585 & 0.00000262 & 2.540413 & 0.01364560 & 1.082020 \\
\hline & 1000 & 0.00022748 & 1.600403 & 0.00000029 & 3.176362 & 0.00476334 & 1.518391 \\
\hline Esq 3 & 125 & 0.00503130 & - & 0.00010263 & - & 0.04469766 & - \\
\hline$\epsilon=0$ & 250 & 0.00214165 & 1.232208 & 0.00002520 & 2.025879 & 0.03894924 & 0.198604 \\
\hline & 500 & 0.00097851 & 1.130062 & 0.00000798 & 1.657773 & 0.02945532 & 0.403067 \\
\hline & 1000 & 0.00035437 & 1.465294 & 0.00000153 & 2.383867 & 0.01624345 & 0.858670 \\
\hline Esq 3 & 125 & 0.00372947 & - & 0.00006231 & - & 0.04805551 & - \\
\hline$\epsilon=0.1$ & 250 & 0.00158672 & 1.232918 & 0.00001335 & 2.222021 & 0.02538450 & 0.920754 \\
\hline & 500 & 0.00065765 & 1.270655 & 0.00000247 & 2.431077 & 0.01513576 & 0.745987 \\
\hline & 1000 & 0.00022011 & 1.579081 & 0.00000026 & 3.223444 & 0.00439491 & 1.784053 \\
\hline Esq 3 & 125 & 0.00449332 & - & 0.00008358 & - & 0.04416584 & - \\
\hline$\epsilon=0.01$ & 250 & 0.00194748 & 1.206175 & 0.00002043 & 2.032188 & 0.03702473 & 0.254442 \\
\hline & 500 & 0.00074645 & 1.383485 & 0.00000337 & 2.597985 & 0.01813971 & 1.029337 \\
\hline & 1000 & 0.00023806 & 1.648706 & 0.00000038 & 3.129180 & 0.00840174 & 1.110390 \\
\hline
\end{tabular}


Tabela 6.8: Continuação da Tabela 6.7.

\begin{tabular}{|c|c|c|c|c|c|c|c|}
\hline & \multirow[t]{2}{*}{$N$} & \multicolumn{2}{|c|}{$L_{1}$} & \multicolumn{2}{|c|}{$L_{2}$} & \multicolumn{2}{|c|}{$L_{\infty}$} \\
\hline & & Erro & $q$ & Erro & $q$ & Erro & $q$ \\
\hline \multirow{4}{*}{$\begin{array}{l}\text { Esq } 4 \\
\epsilon=0\end{array}$} & 125 & 0.00419579 & - & 0.00007295 & - & 0.04331085 & - \\
\hline & 250 & 0.00180007 & 1.220887 & 0.00001588 & 2.199750 & 0.03061687 & 0.500402 \\
\hline & 500 & 0.00069091 & 1.381475 & 0.00000261 & 2.603323 & 0.01404149 & 1.124630 \\
\hline & 1000 & 0.00022895 & 1.593432 & 0.00000029 & 3.159537 & 0.00493815 & 1.507653 \\
\hline \multirow{4}{*}{$\begin{array}{l}\text { Esq } 4 \\
\epsilon=0.1\end{array}$} & 125 & 0.00409389 & - & 0.00006968 & - & 0.04150062 & - \\
\hline & 250 & 0.00175941 & 1.218384 & 0.00001503 & 2.212550 & 0.02869001 & 0.532584 \\
\hline & 500 & 0.00068308 & 1.364951 & 0.00000254 & 2.564496 & 0.01287757 & 1.155688 \\
\hline & 1000 & 0.00022781 & 1.584175 & 0.00000029 & 3.128099 & 0.00491000 & 1.391066 \\
\hline \multirow{4}{*}{$\begin{array}{l}\text { Esq } 4 \\
\epsilon=0.01\end{array}$} & 125 & 0.00418441 & - & 0.00007257 & - & 0.04312864 & - \\
\hline & 250 & 0.00179561 & 1.220551 & 0.00001577 & 2.201367 & 0.03040407 & 0.504382 \\
\hline & 500 & 0.00068992 & 1.379963 & 0.00000260 & 2.599238 & 0.01390973 & 1.128170 \\
\hline & 1000 & 0.00022884 & 1.592086 & 0.00000029 & 3.155085 & 0.00493746 & 1.494253 \\
\hline \multirow{4}{*}{$\begin{array}{l}\text { Esq } 5 \\
\epsilon=0\end{array}$} & 125 & 0.00382996 & - & 0.00006374 & - & 0.04211336 & - \\
\hline & 250 & 0.00162764 & 1.234549 & 0.00001277 & 2.318668 & 0.02391000 & 0.816663 \\
\hline & 500 & 0.00066620 & 1.288745 & 0.00000285 & 2.161517 & 0.01872921 & 0.352325 \\
\hline & 1000 & 0.00023172 & 1.523582 & 0.00000038 & 2.878042 & 0.00831988 & 1.170654 \\
\hline \multirow{4}{*}{$\begin{array}{l}\text { Esq } 5 \\
\epsilon=0.1\end{array}$} & 125 & 0.00383349 & - & 0.00006390 & - & 0.04227434 & - \\
\hline & 250 & 0.00162894 & 1.234724 & 0.00001279 & 2.320431 & 0.02369738 & 0.835054 \\
\hline & 500 & 0.00066555 & 1.291302 & 0.00000281 & 2.181737 & 0.01858748 & 0.350396 \\
\hline & 1000 & 0.00023053 & 1.529601 & 0.00000037 & 2.894476 & 0.00822589 & 1.176087 \\
\hline \multirow{4}{*}{$\begin{array}{l}\text { Esq } 5 \\
\epsilon=0.01\end{array}$} & 125 & 0.00383035 & - & 0.00006376 & - & 0.04213017 & - \\
\hline & 250 & 0.00162778 & 1.234567 & 0.00001278 & 2.318863 & 0.02388817 & 0.818557 \\
\hline & 500 & 0.00066613 & 1.289019 & 0.00000285 & 2.163594 & 0.01871500 & 0.352101 \\
\hline & 1000 & 0.00023159 & 1.524197 & 0.00000038 & 2.879706 & 0.00831053 & 1.171183 \\
\hline \multirow{4}{*}{$\begin{array}{l}\text { Esq } 6 \\
\epsilon=0\end{array}$} & 125 & 0.00477964 & - & 0.00009065 & - & 0.04287391 & - \\
\hline & 250 & 0.00220619 & 1.115345 & 0.00002768 & 1.711242 & 0.03842228 & 0.158157 \\
\hline & 500 & 0.00096120 & 1.198648 & 0.00000701 & 1.980098 & 0.02604985 & 0.560668 \\
\hline & 1000 & 0.00039813 & 1.271568 & 0.00000202 & 1.793405 & 0.01647076 & 0.661368 \\
\hline \multirow{4}{*}{$\begin{array}{l}\text { Esq } 6 \\
\epsilon=0.1\end{array}$} & 125 & 0.00419182 & - & 0.00007138 & - & 0.04164444 & - \\
\hline & 250 & 0.00186052 & 1.171869 & 0.00001728 & 2.046575 & 0.03165554 & 0.395666 \\
\hline & 500 & 0.00074834 & 1.313927 & 0.00000346 & 2.319257 & 0.01863504 & 0.764440 \\
\hline & 1000 & 0.00023880 & 1.647875 & 0.00000038 & 3.154688 & 0.00753245 & 1.306827 \\
\hline \multirow{4}{*}{$\begin{array}{l}\text { Esq } 6 \\
\epsilon=0.01\end{array}$} & 125 & 0.00465479 & - & 0.00008597 & - & 0.04272254 & - \\
\hline & 250 & 0.00214224 & 1.119593 & 0.00002577 & 1.737759 & 0.03775591 & 0.178295 \\
\hline & 500 & 0.00092762 & 1.207510 & 0.00000641 & 2.005512 & 0.02483136 & 0.604539 \\
\hline & 1000 & 0.00036462 & 1.347131 & 0.00000159 & 2.008434 & 0.01521771 & 0.706412 \\
\hline
\end{tabular}

e $\rho_{l}>\rho_{r}$; e a velocidade $u$ é tomada como nula. Este problema é interessante porque sua condição inicial provoca uma onda de rarefação à esquerda de $x_{o}$, além de uma onda de choque e uma descontinuidade de contato à direita deste ponto. A condição inicial considerada foi

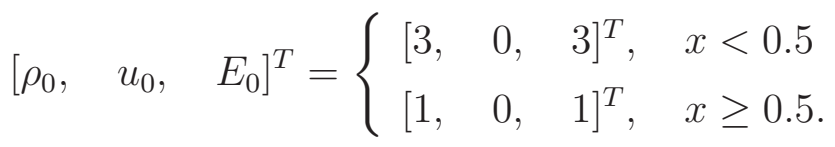

As Figuras 6.30-6.35 apresentam as soluções obtidas considerando uma malha de 300 células 
computacionais para um número de Courant $\theta=0.9, \gamma=1.4$ e um tempo final $T=0.1$ para o melhor $\beta$ e os melhores $\epsilon$. A solução de referência foi calculada pelo método de Godunov de segunda ordem, utilizando o limitador de fluxo do esquema MC (monotonized centered) tomando 1000 células computacionais. Nota-se que o Esquema 1 apresentou, em linhas gerais, a melhor solução dentre os esquemas testados.

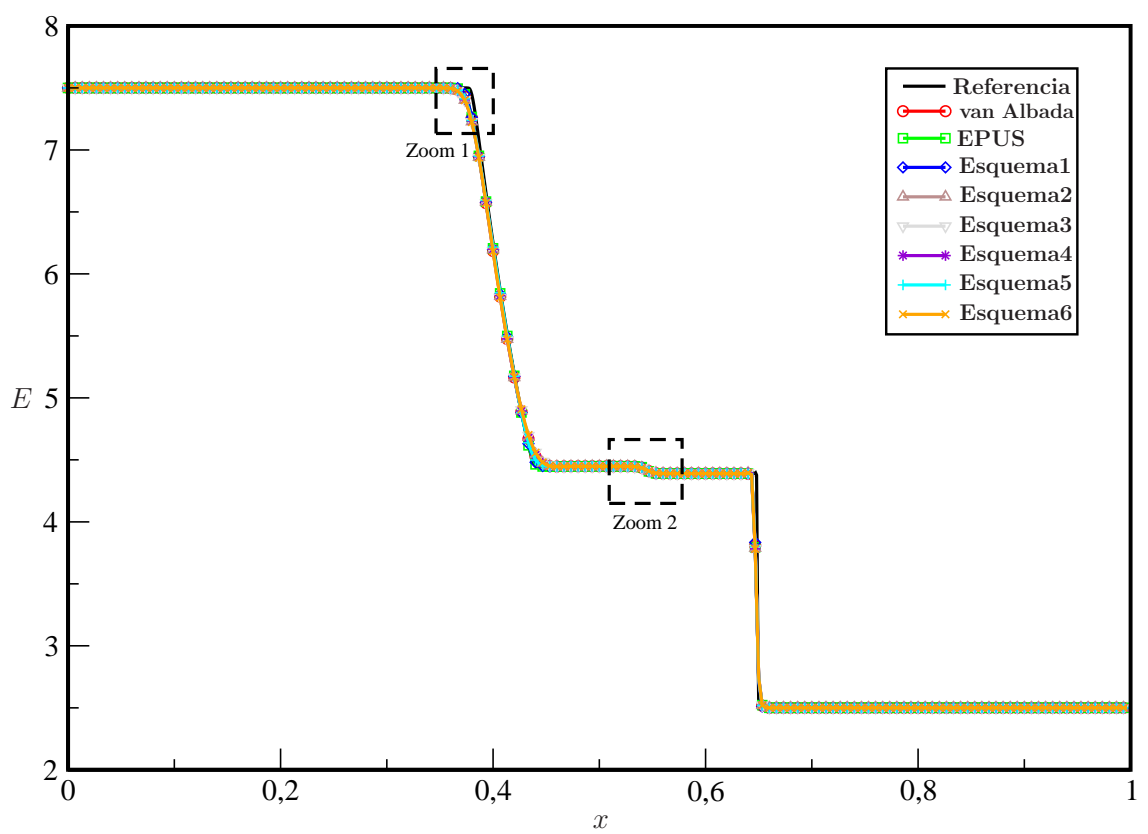

Figura 6.30: Comparação das soluções numéricas e a solução de referência para a energia $E$ no Teste 8, usando os esquema van Albada, EPUS e Esquemas 1-6 com os melhores parâmetros, mostrando regiões de ampliação.

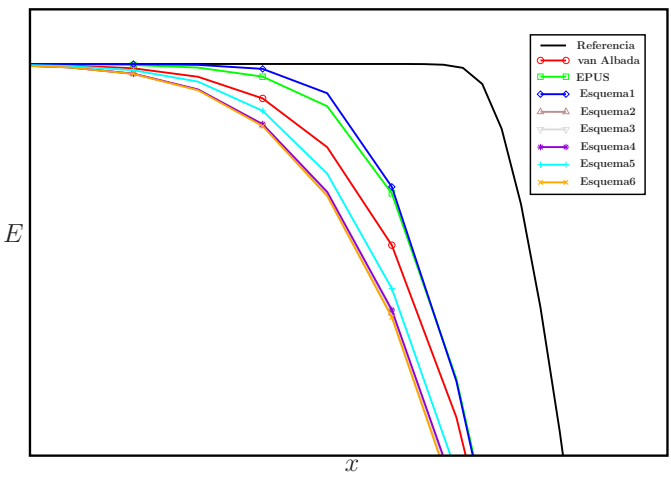

(a) Zoom 1

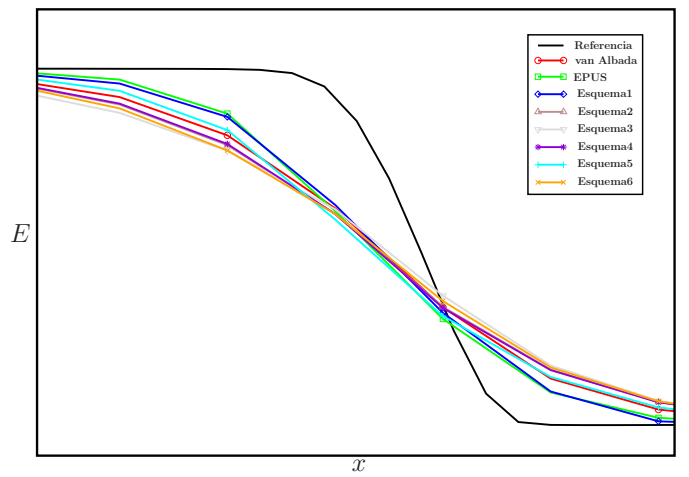

(b) Zoom 2

Figura 6.31: Continuação da Figura 6.30.

Na Figura 6.36 mostra-se a variação TV para os esquemas da família FUS-RF. A variação foi calculada para três malhas distintas, $N=10, N=25$ e $N=50$. Todos os esquemas comportaram-se de maneira bem parecida, mostrando-se TV para os melhores valores de $\beta$ e $\epsilon$. 


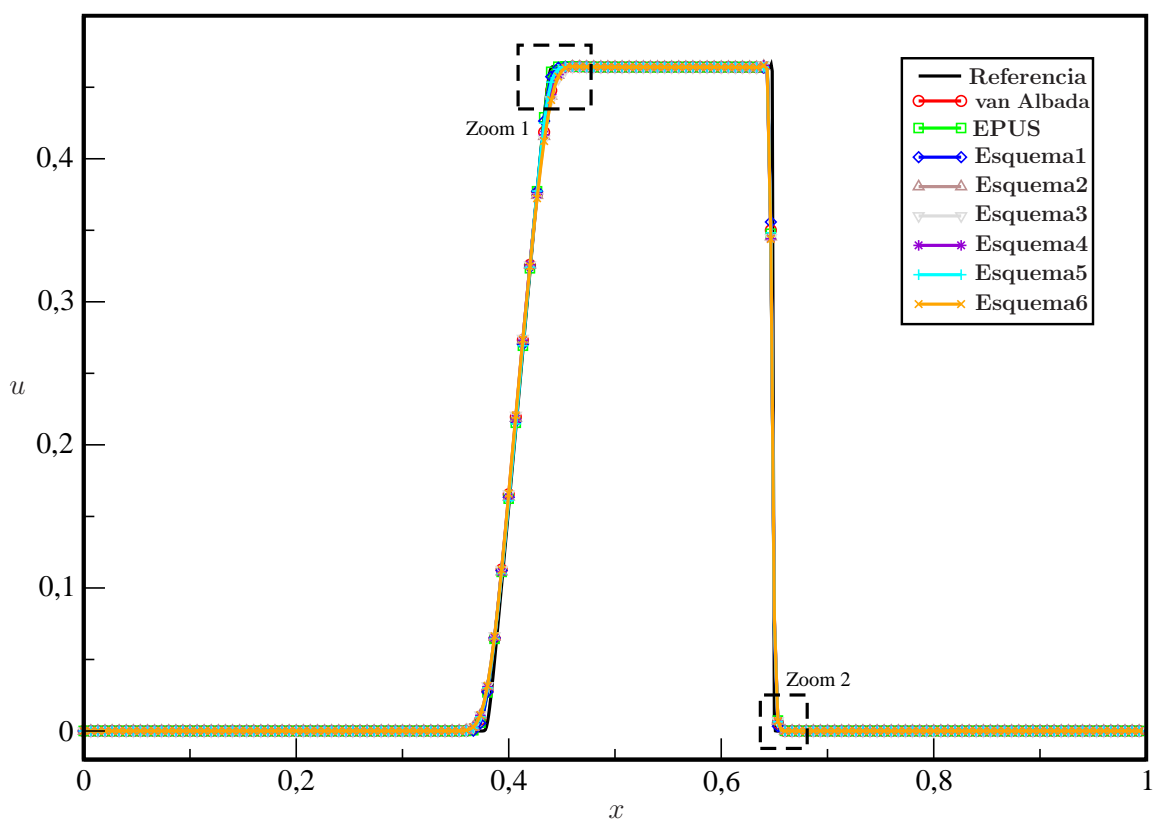

Figura 6.32: Comparação das soluções numéricas e a solução de referência para a velocidade $u$ no Teste 8, usando os esquema van Albada, EPUS e Esquemas 1-6 com os melhores parâmetros, mostrando regiões de ampliação.

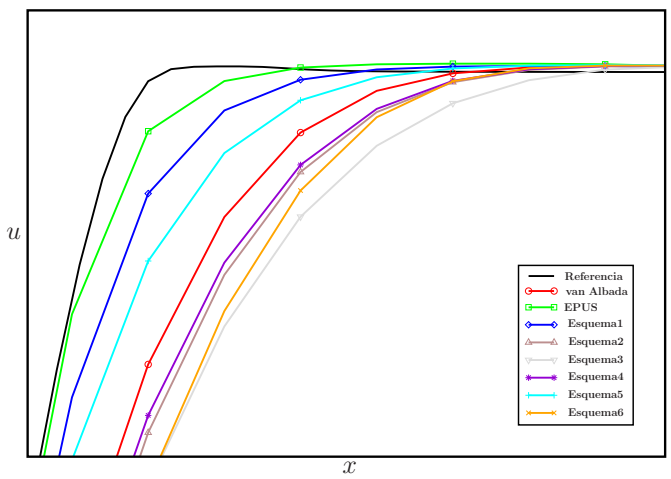

(a) Zoom 1

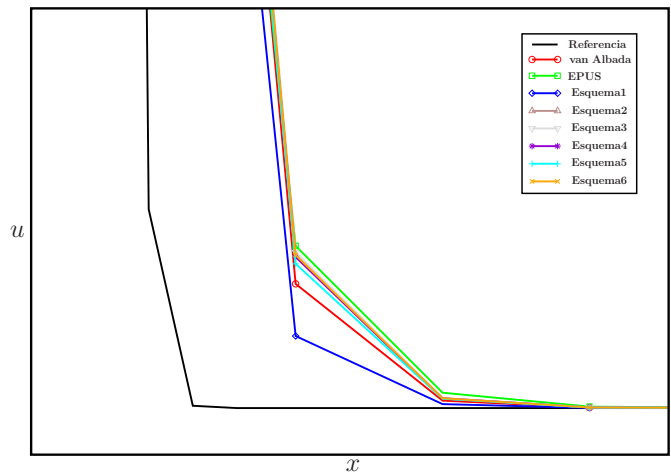

(b) Zoom 2

Figura 6.33: Continuação da Figura 6.32.

Teste 9 (Two Interacting Blast Waves): Este problema foi proposto por Wood e Collela [53] e, assim como no Teste 8, este problema se caracteriza pela formação de ondas de choque, de contato e de rarefação. Ele é caracterizado por três regiões de descontinuidade para a pressão $p$. Na primeira região assume-se uma alto gradiente de pressão. Na segunda região este gradiente é significantemente reduzido, e é substancialmente acrescido na região seguinte. Esta variação de pressão provoca o deslocamento de duas ondas de choque, que se propagam em direções opostas provocando o choque (a primeira viaja da região 1 para a região 2 e a segunda da região 3 para a 2).

Como condição inicial temos: 


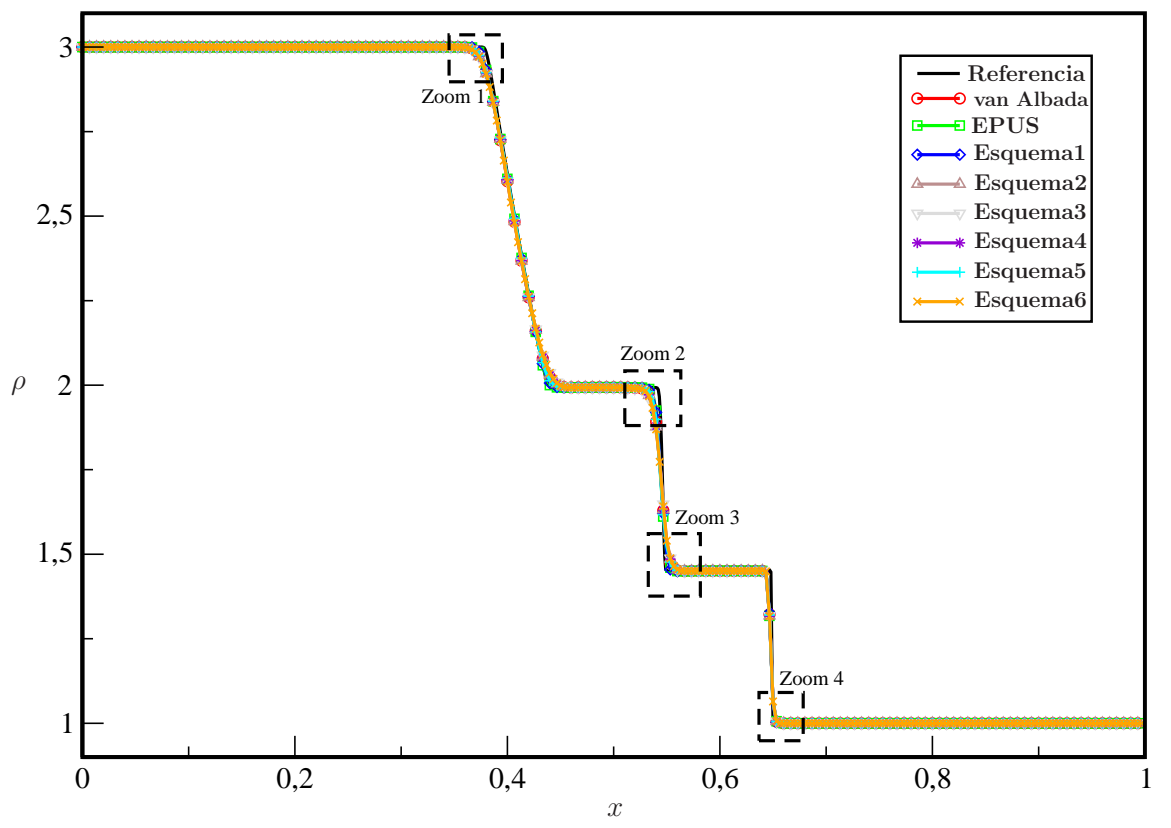

Figura 6.34: Comparação das soluções numéricas e a solução de referência para a densidade $\rho$ no Teste 8, usando os esquema van Albada, EPUS e Esquemas 1-6 com os melhores parâmetros, mostrando regiões de ampliação.

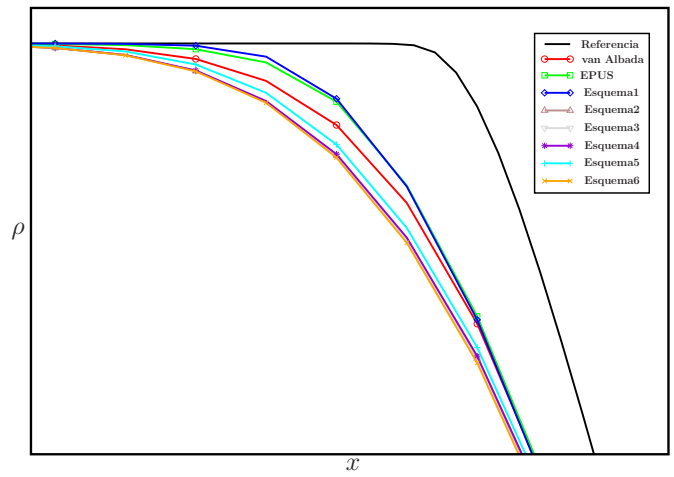

(a) Zoom 1

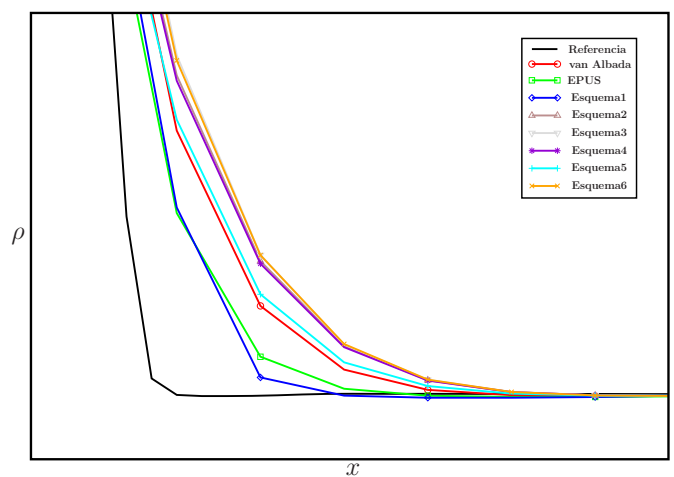

(a) Zoom 3

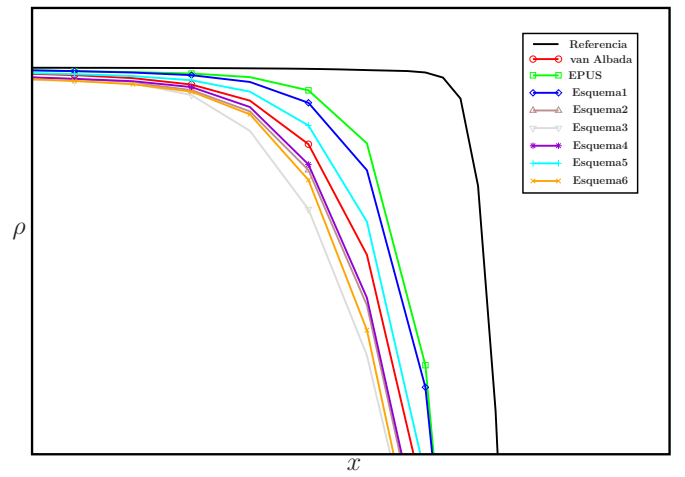

(b) Zoom 2

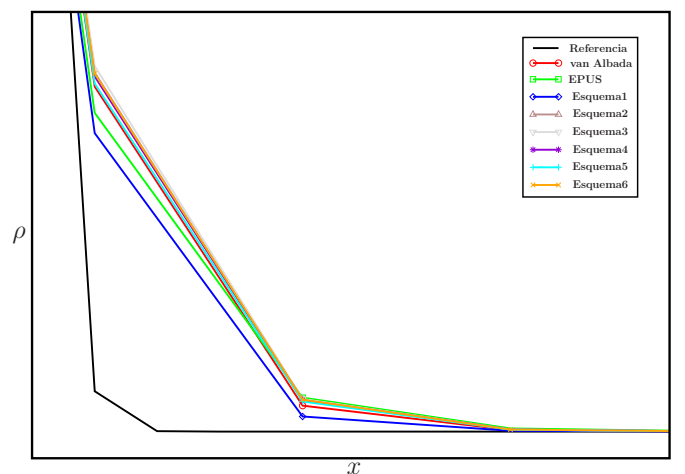

(b) Zoom 4

Figura 6.35: Continuação da Figura 6.34. 
(a) Esquema $1 \beta=0.1545$

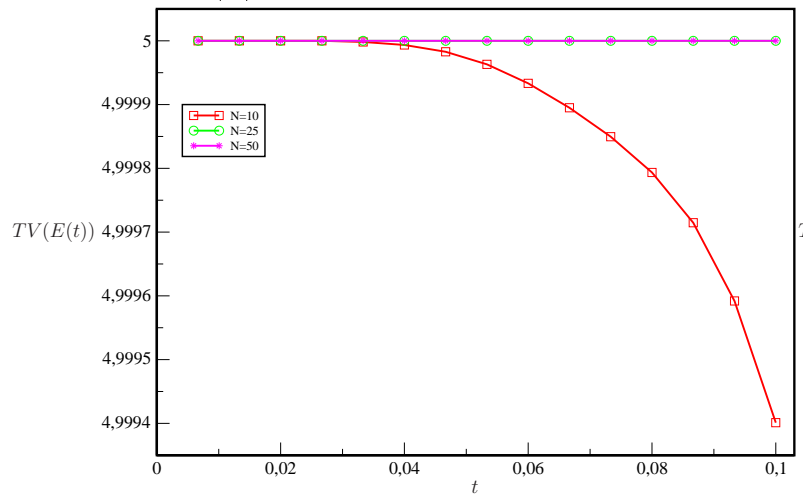

(c) Esquema $3 \epsilon=10^{-} 2$

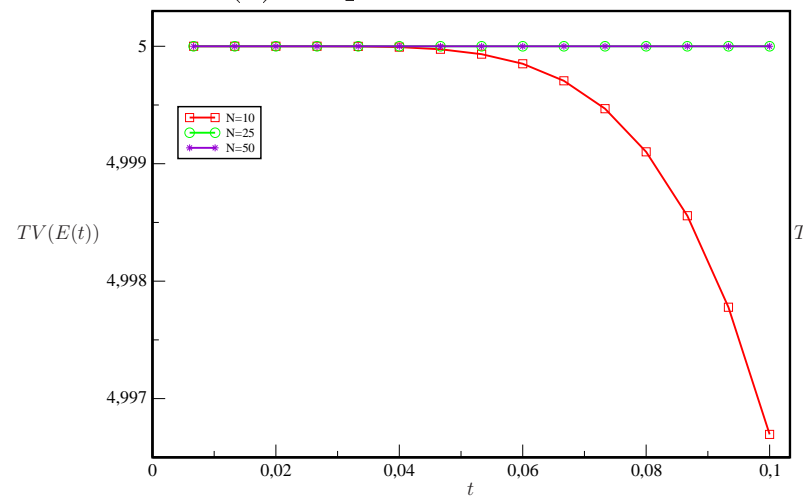

(e) Esquema $5 \epsilon=0$

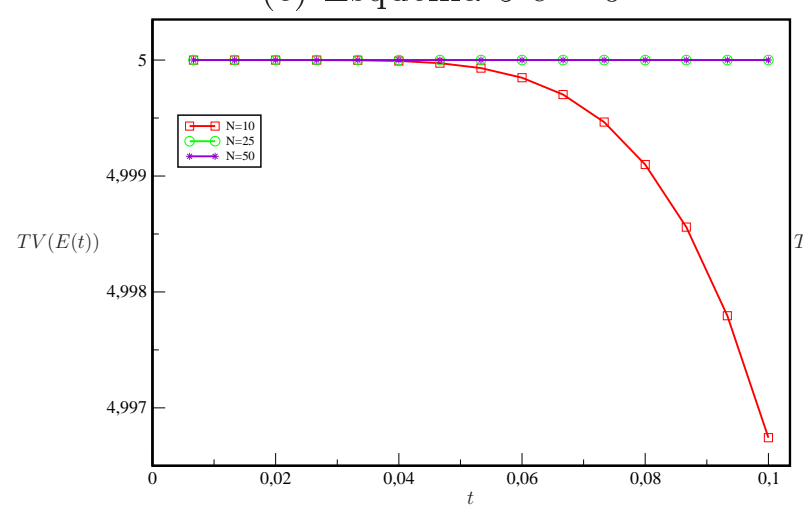

(b) Esquema $2 \epsilon=10^{-} 2$

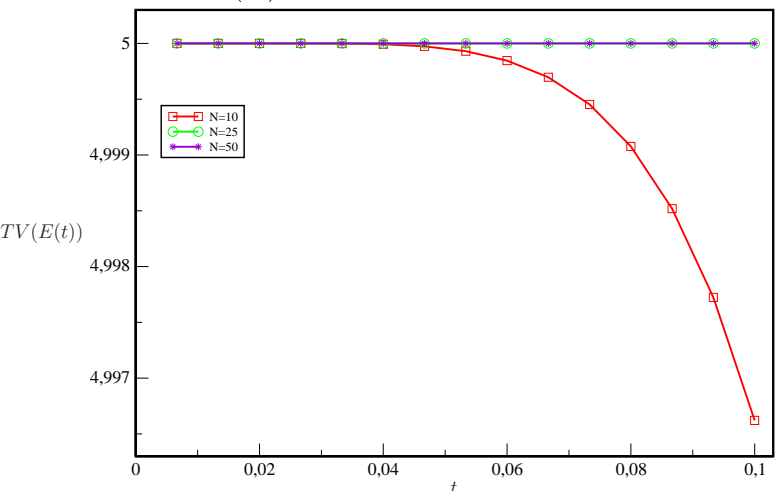

(d) Esquema $4 \epsilon=10^{-} 1$

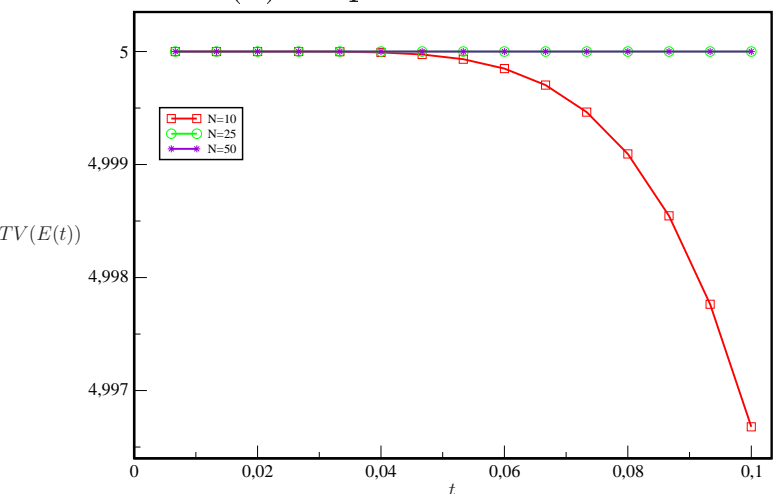

(f)Esquema $6 \epsilon=10^{-} 1$

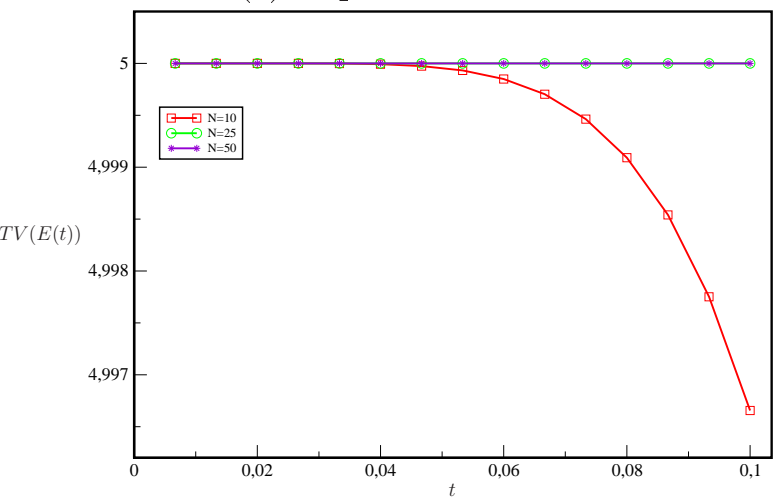

Figura 6.36: Variação total da variável conservada $E$ para o Teste 8 em várias malhas com respeito ao tempo.

$$
\left[\rho_{0}, \quad u_{0}, \quad E_{0}\right]^{T}=\left\{\begin{array}{lll}
{\left[\begin{array}{lll}
1, & 0, & \frac{1000}{\gamma}
\end{array}\right]^{T},} & 0 \leq x \geq 0.1 \\
{\left[\begin{array}{lll}
1, & 0, & \frac{0.01}{\gamma}
\end{array}\right]^{T},} & 0.1<x \geq 0.9 \\
{\left[\begin{array}{lll}
1, & 0, & \frac{100}{\gamma}
\end{array}\right]^{T},} & 0.9<x<\geq 1.0
\end{array}\right.
$$

Na simulação foi considerada uma malha de $N=700$ células tomadas em $[0,1]$ para $C F L=$ 0.9 e $T=0.038$. Nas Figuras 6.37-6.42 vê-se as soluções para $p, \rho$ e $u$ e as regiões de ampliação 
(zoom). Nota-se que o Esquema 1 obteve o melhor resultado, dentre os seis esquemas propostos.

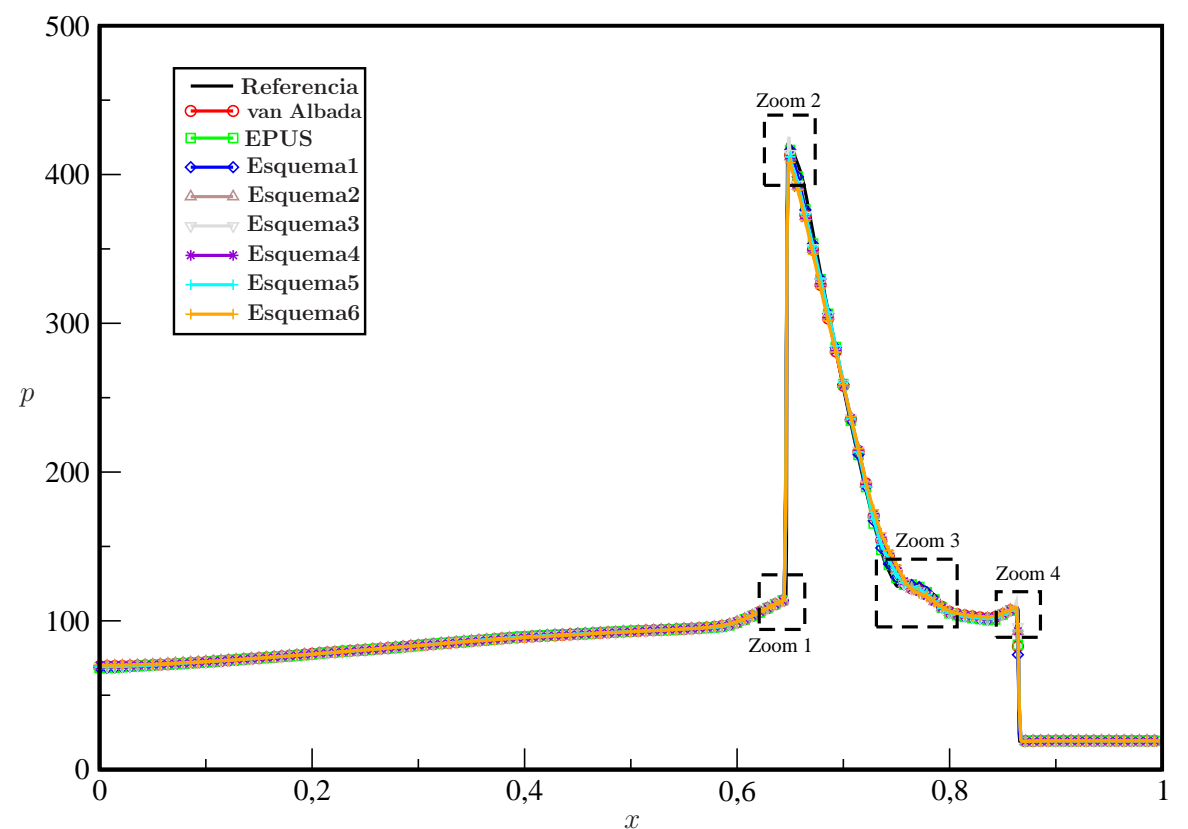

Figura 6.37: Comparação das soluções numéricas e a solução de referência para a pressão $p$ no Teste 9, usando os esquema van Albada, EPUS e Esquemas 1-6 com os melhores parâmetros, mostrando regiões de ampliação.

(a) Zoom 1

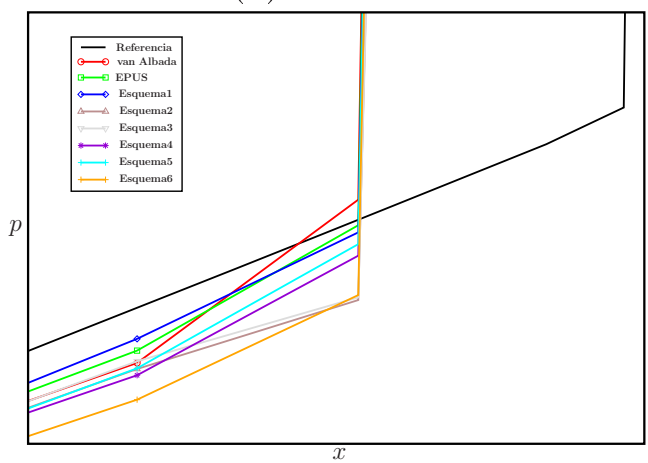

(a) Zoom 3

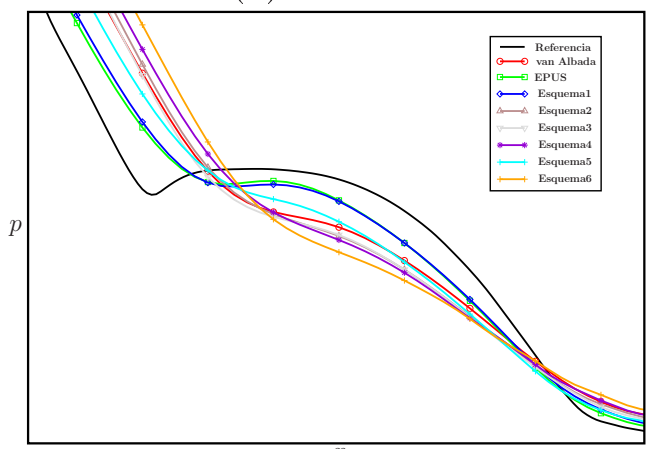

(b) Zoom 2

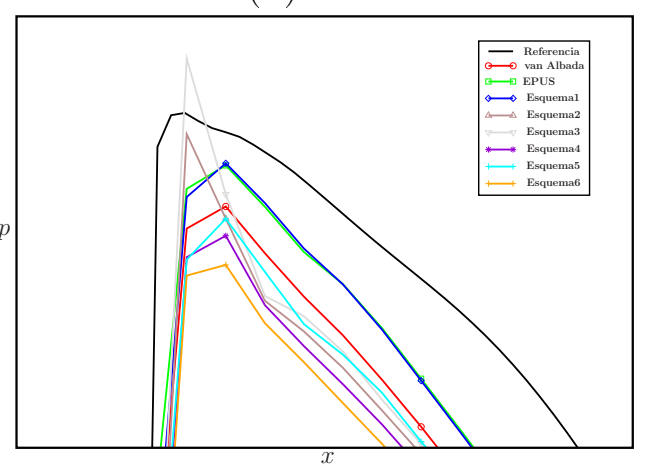

(b) Zoom 4

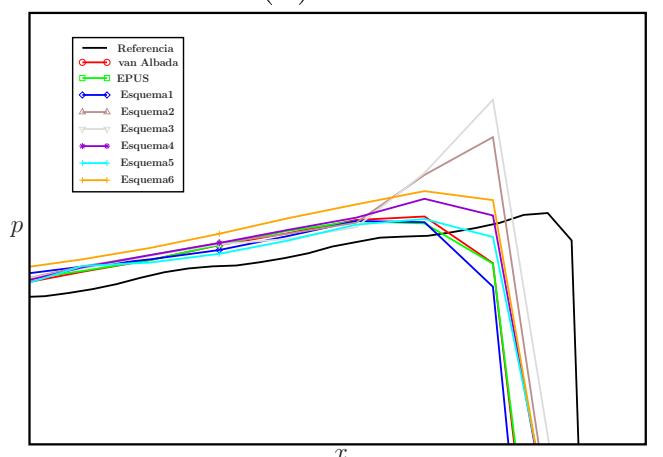

Figura 6.38: Continuação da Figura 6.37. 


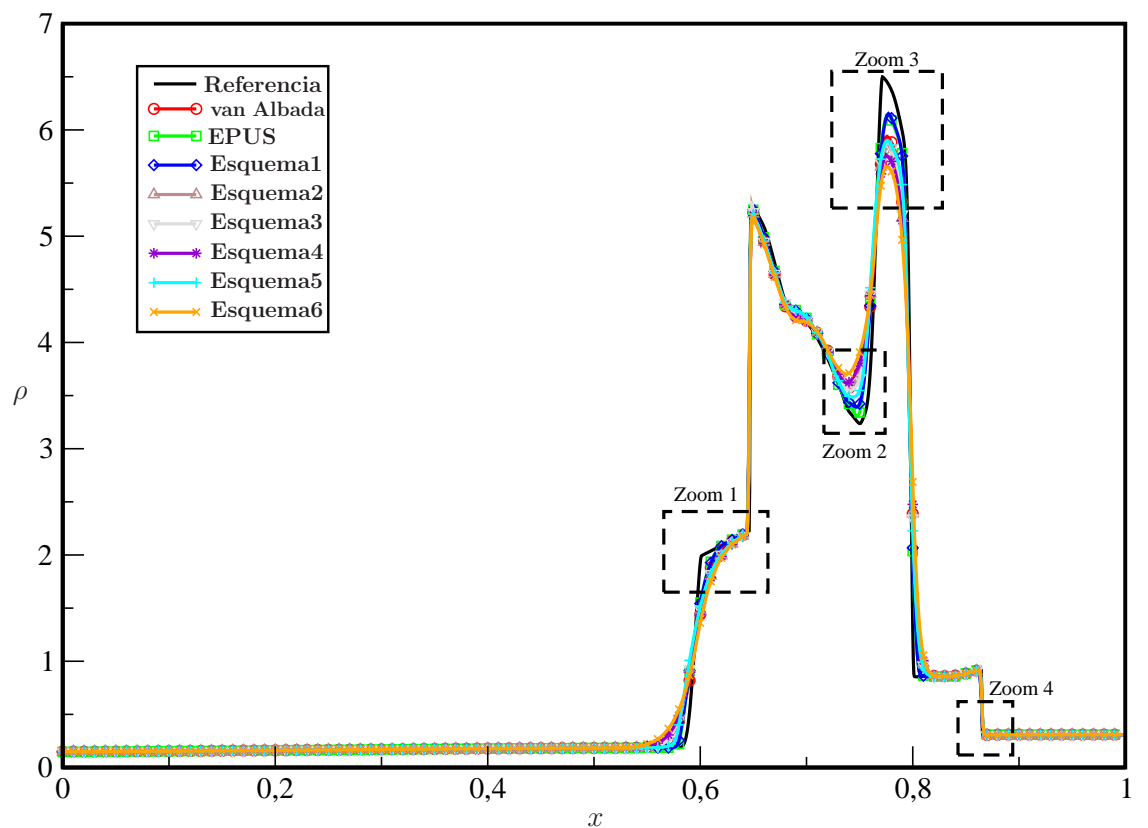

Figura 6.39: Comparação das soluções numéricas e a solução de referência para a densidade $\rho$ no Teste 9, usando os esquema van Albada, EPUS e Esquemas 1-6 com os melhores parâmetros, mostrando regiões de ampliação.

(a) Zoom 1

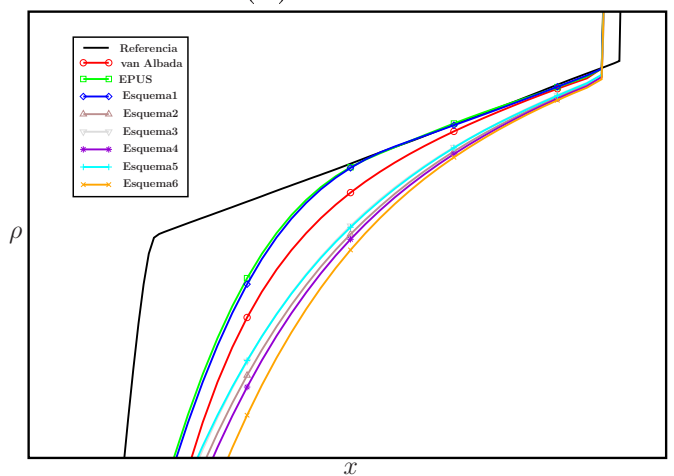

(a) Zoom 3

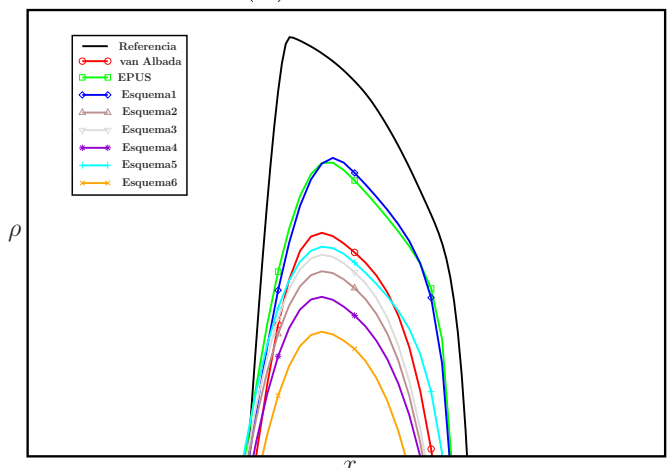

(b) Zoom 2

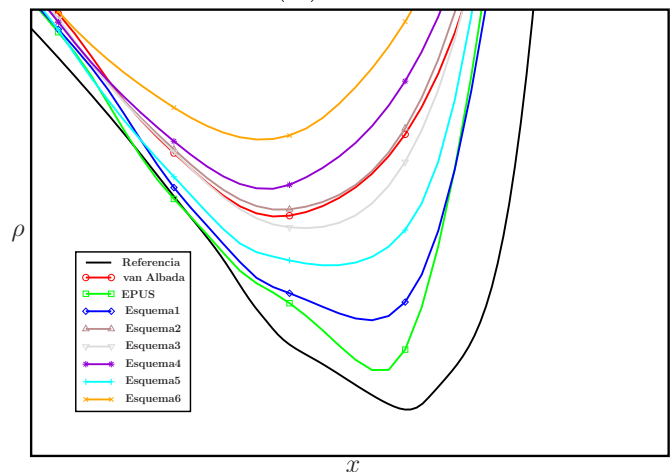

(b) Zoom 4

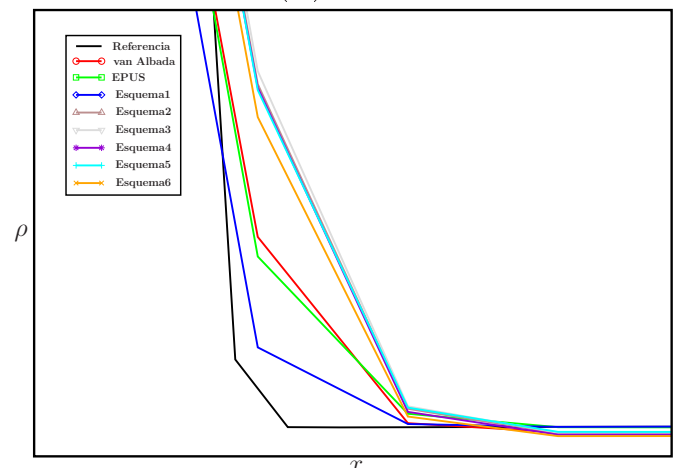

Figura 6.40: Continuação da Figura 6.39. 


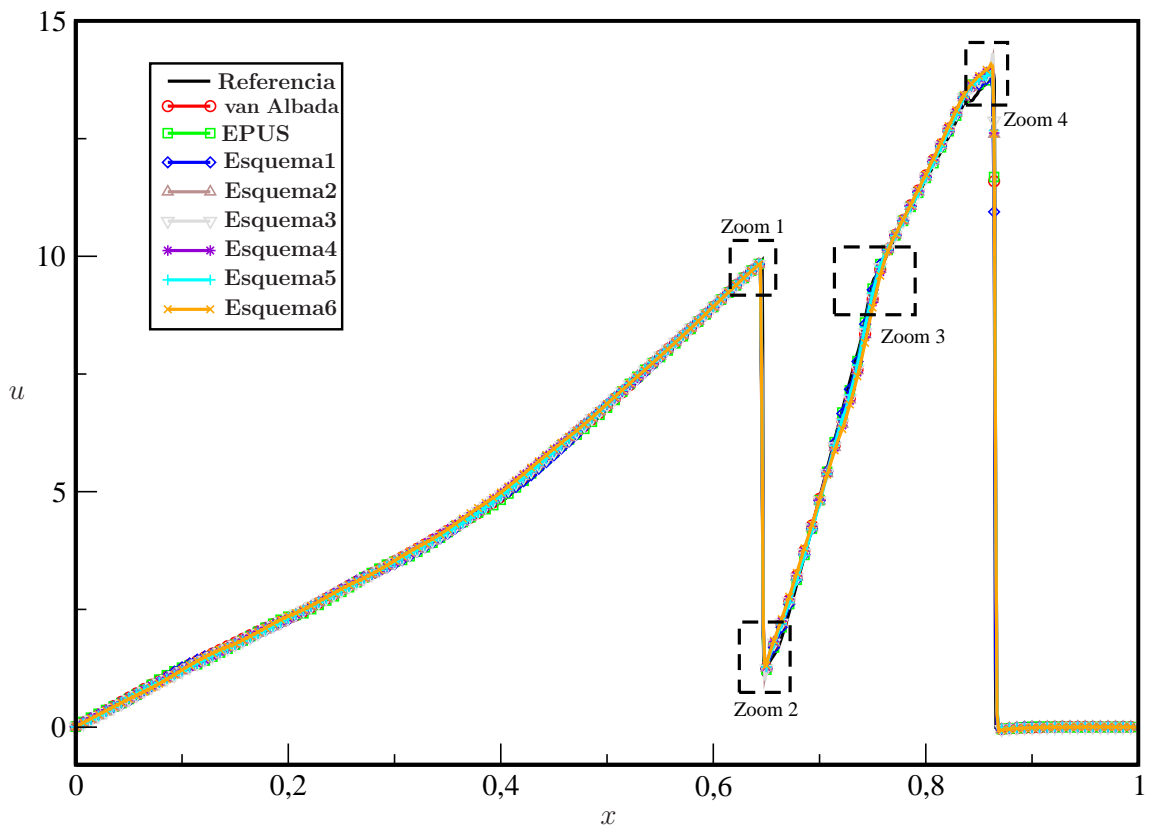

Figura 6.41: Comparação das soluções numéricas e a solução de referência para a velocidade $u$ no Teste 9, usando os esquema van Albada, EPUS e Esquemas 1-6 com os melhores parâmetros, mostrando regiões de ampliação.

(a) Zoom 1

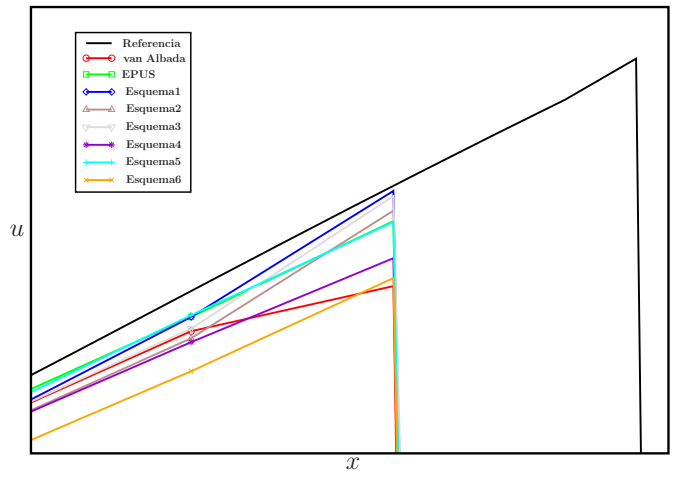

(a) Zoom 3

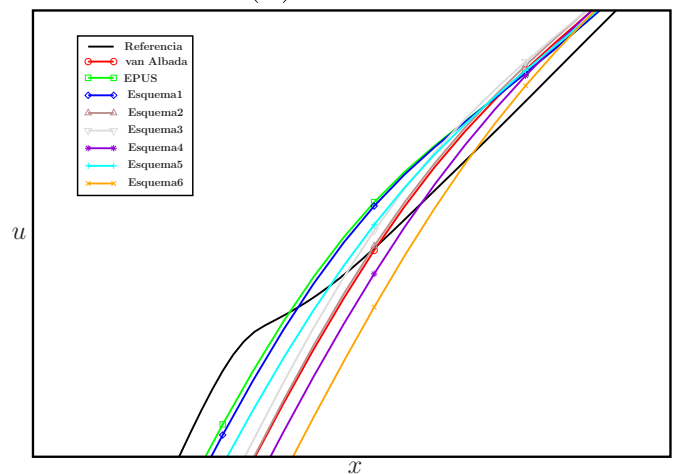

(b) Zoom 2

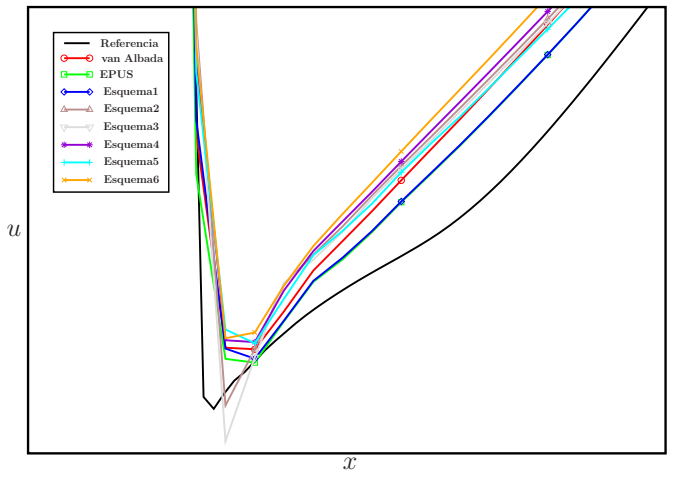

(b) Zoom 4

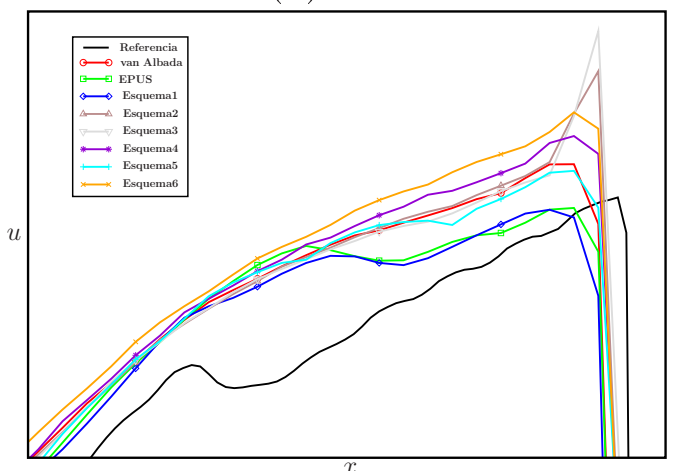

Figura 6.42: Continuação da Figura 6.41. 


\subsection{Resultados para equações de águas rasas 2D}

Simula-se nesta seção o rompimento de uma barreira cilíndrica, modelado pelas equações de águas rasas 2D;

Teste 10( Radial dam-break problem): O problema consiste numa porção cilíndrica de fluido retida por uma barreira e instantaneamente removida. Isto provoca uma mudança abrupta no movimento do fluido e provoca a formação de duas ondas que se propagam em direções opostas; uma propagando-se radialmente em direção à região de menor altura e a outra (onda de rarefação) propagando-se para o interior da região cilíndrica.

A Figura 6.43 (extraída de LeVeque [21]) apresenta a solução inicial e a formação das ondas de choque e rarefação no tempo $t=0.25$. Na simulação numérica definiu-se uma malha de $200 \times 200$ células computacionais no domínio $[-2.5,2.5] \times[-2.5,2.5]$. Foram usados o número de Courant $\theta=0.9$ e tempo final de simulação $T=1.5$. Como solução de referência foi escolhida a solução numérica $1 D$ (ver LeVeque [21]) utilizando 2000 células computacionais. Como condição inicial assume-se o raio $r_{0}=0.5 ; h=2$ para a região pertencente ao interior do cilindro e $h=1$ fora desta região. Assume-se ainda que o fluido está em repouso $\left(u_{0}=0\right.$ e $\left.v_{0}=0\right)$.
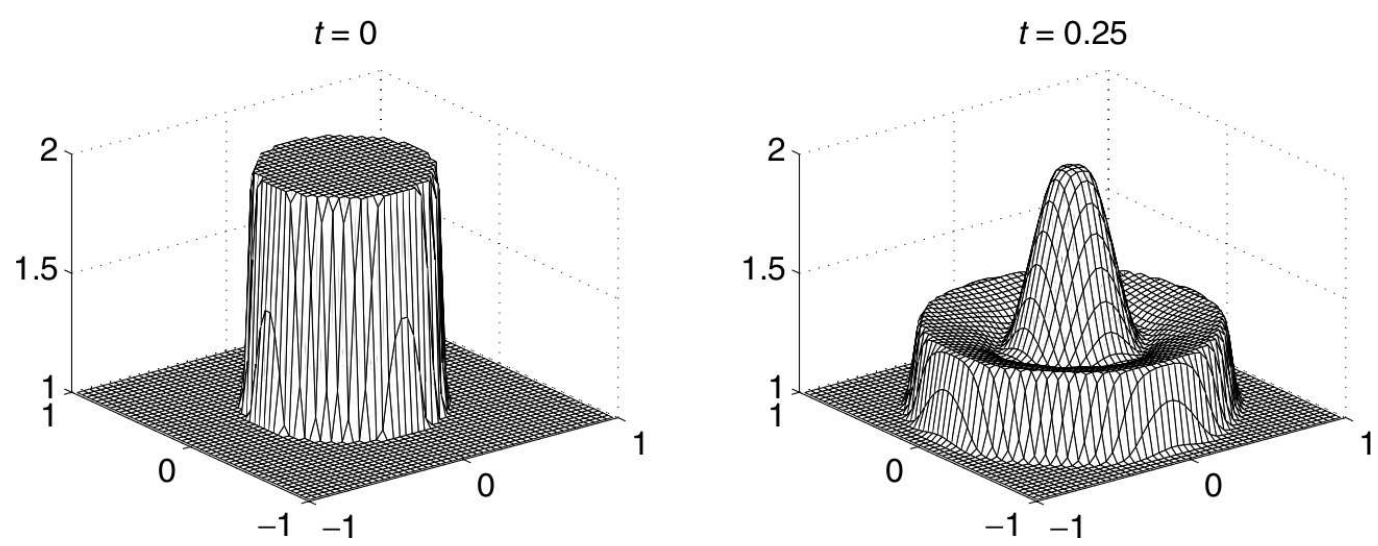

Figura 6.43: Comportamento da altura $h$ da porção de fluido nos instantes $t=0$ e $t=0.25$. Figura extraída de LeVeque [21]

Na Figura 6.44 é apresentada, ao longo da reta $y=0$, a comparação entre as soluções obtidas pelos esquemas da família FUS-RF para distintos valores de $\beta(\beta=0.1545,0.1605,0.1670)$ e de $\epsilon(\epsilon=0,0.1,0.01)$ e a solução de referência. O Esquema 1 apresentou melhor resultado $\operatorname{com} \beta=0.1545$, enquanto os Esquemas 2 e 3 foram melhores com $\epsilon=0.01$. O Esquema 5 não apresentou mudança significativa com a variação de $\epsilon$; já os Esquemas 4 e 6 foram melhores com $\epsilon=0.1$. Na Figura 6.45 apresentam-se uma comparação das soluções obtidas com os esquemas FUS-RF, EPUS e van Albada com a solução de referência. Vê-se que o Esquema 1 e o EPUS 
forneceram os melhores resultados.

(a) Esquema 1

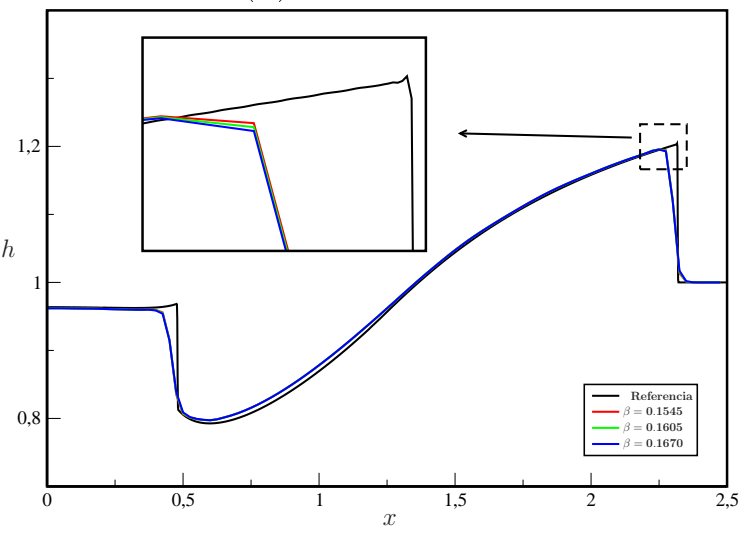

(c) Esquema 3

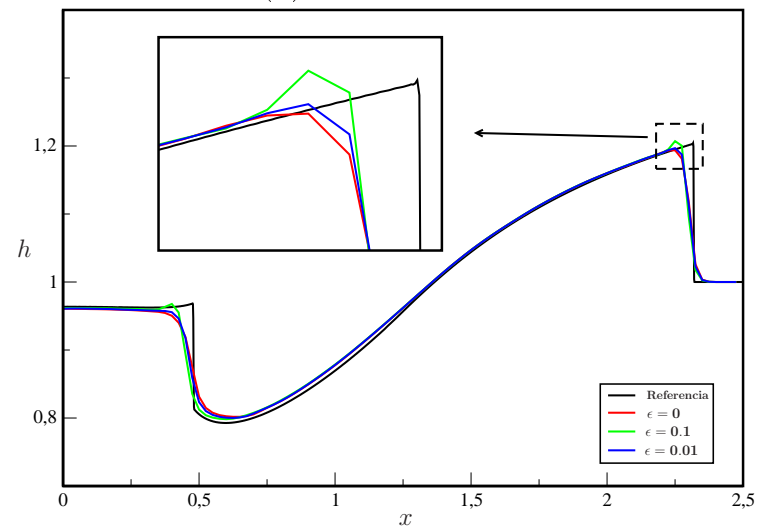

(e) Esquema 5

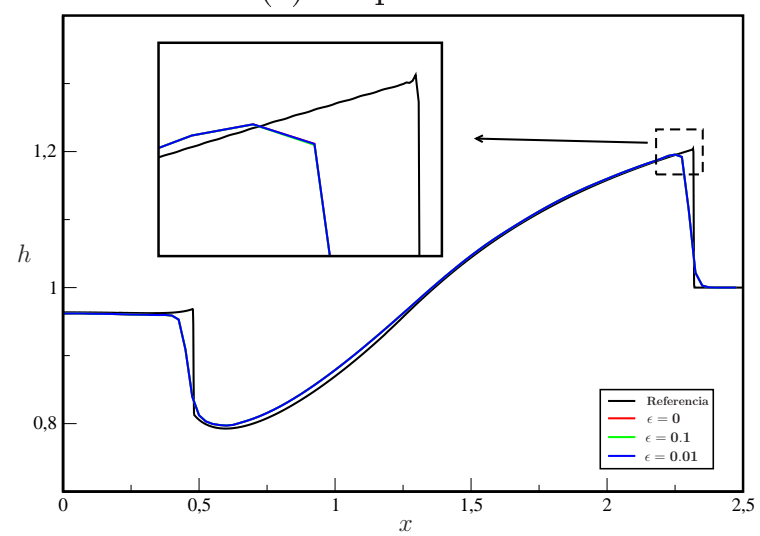

(b) Esquema 2

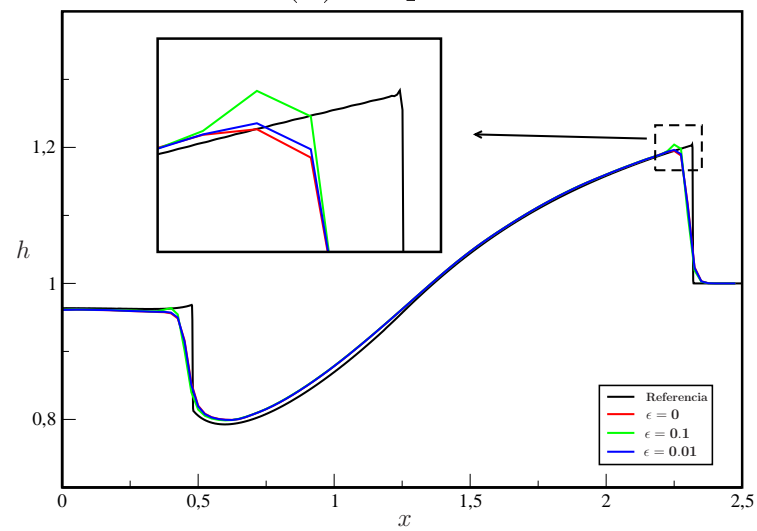

(d) Esquema 4

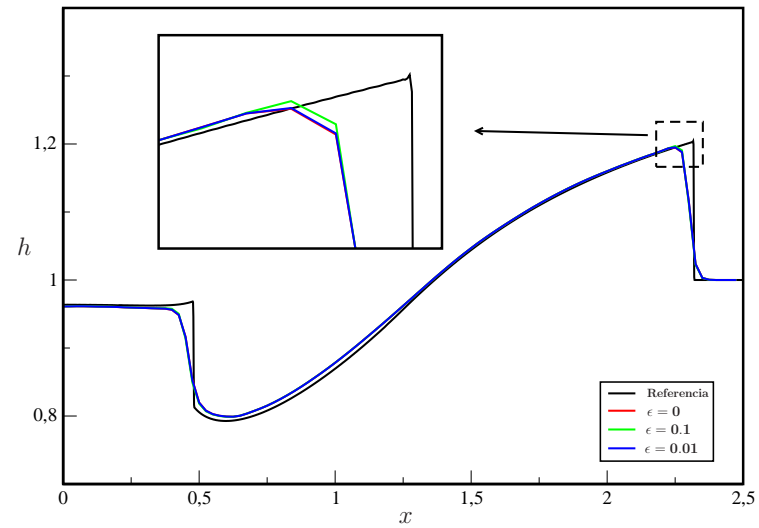

(f) Esquema 6

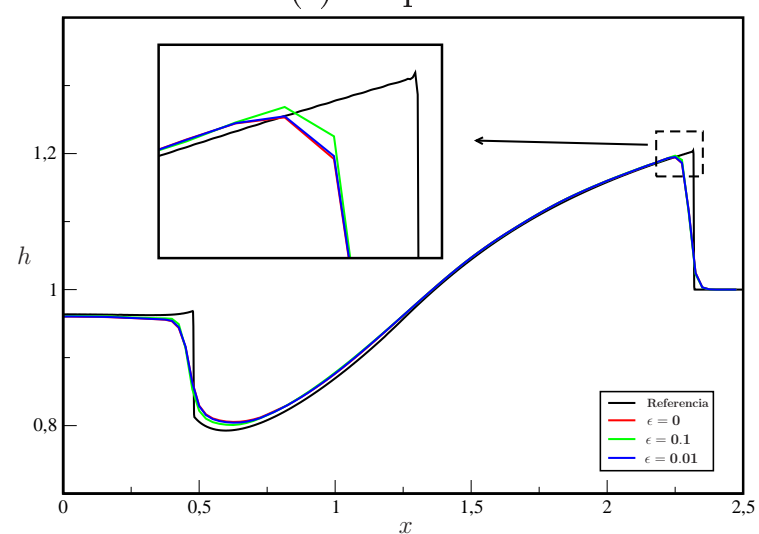

Figura 6.44: Solução de referência e resultados obtidos com os esquemas FUS-RF sob a reta $y=0$. (a) Esquema 1 utilizando vários valores do parâmetro $\beta$; (b)-(f) Esquemas 2-6 utilizando vários valores do parâmetro $\epsilon$. 


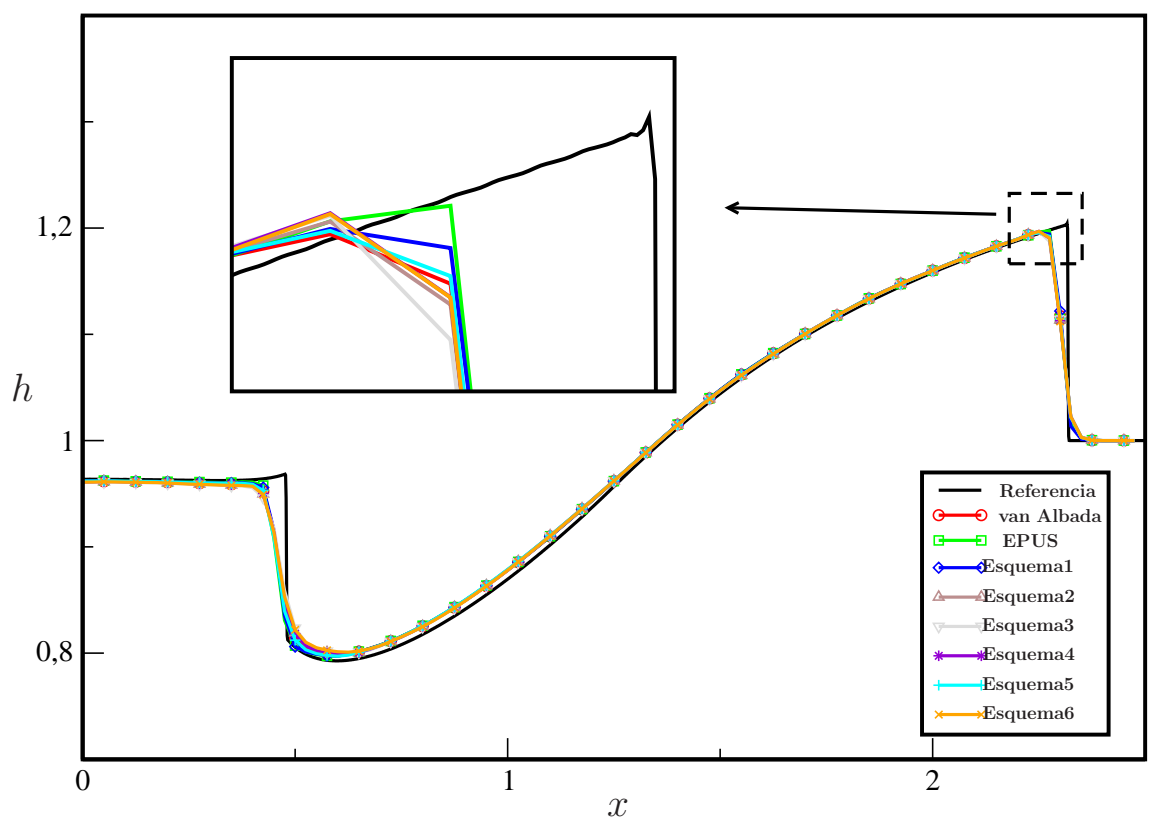

Figura 6.45: Solução de referência e resultados numéricos (Esquemas 1-6 com os melhores parâmetros, van Albada e EPUS) para o Teste 10, mostrando região de ampliação.

Nas Figuras 6.46 e 6.47 tem-se o perfil da variação da altura $h$ no plano $x \perp y$ em $t=1.5$ para os melhores valores dos parâmetros $\beta$ e $\epsilon$. Nota-se que o problema do rompimento de uma barreira cilíndrica foi simulado com sucesso com todos os esquemas estudados.

\subsection{Resultados para as equações de Euler 2D}

Nesta seção são apresentados resultados computacionais para 3 problemas modelados pelas equações de Euler 2D (2.9). O primeiro deles é um problema interessante com solução conhecida. O segundo é um problema difícil de se simular, conhecido como Four-Shocks, e o terceiro contempla instabilidades de Rayleigh-Taylor.

Teste 11: Este teste tem o objetivo de investigar a solução numérica dos esquemas FUS-RF em comparação com a solução analítica.

A condição inicial é dada por

$$
\left\{\begin{array}{l}
\rho(x, y, 0)=1+0.2 \sin (\pi(x+y)) \\
u(x, y, 0)=0.7 \\
v(x, y, 0)=0.3 \\
p(x, y, 0)=1.0 .
\end{array}\right.
$$


(a) van Albada

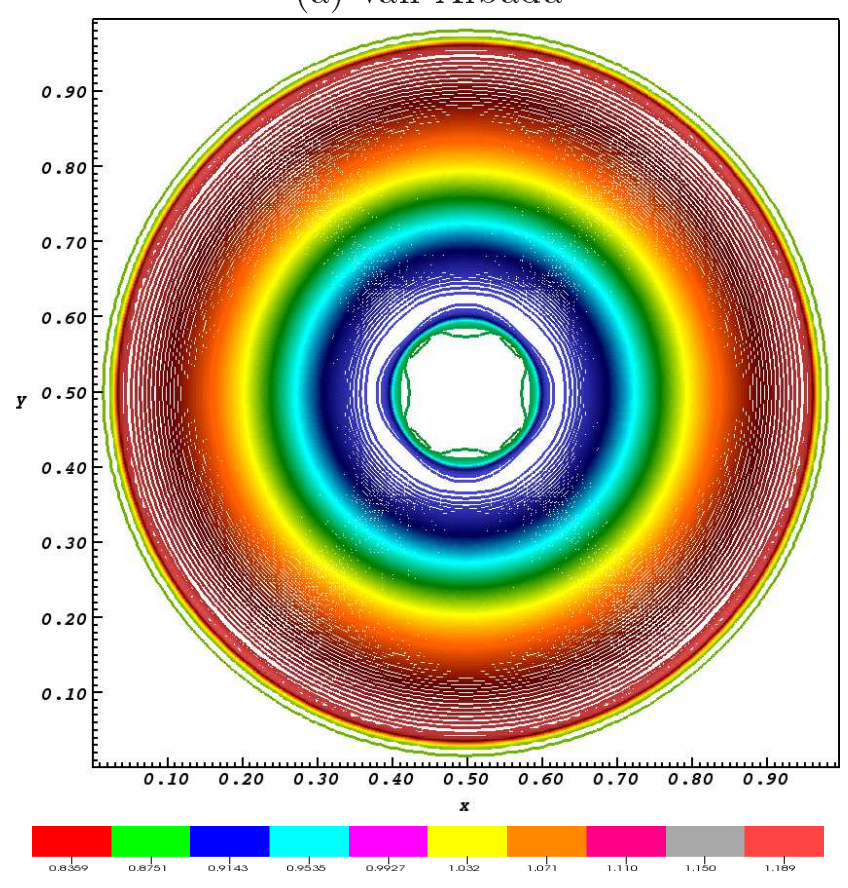

(c) Esquema 1

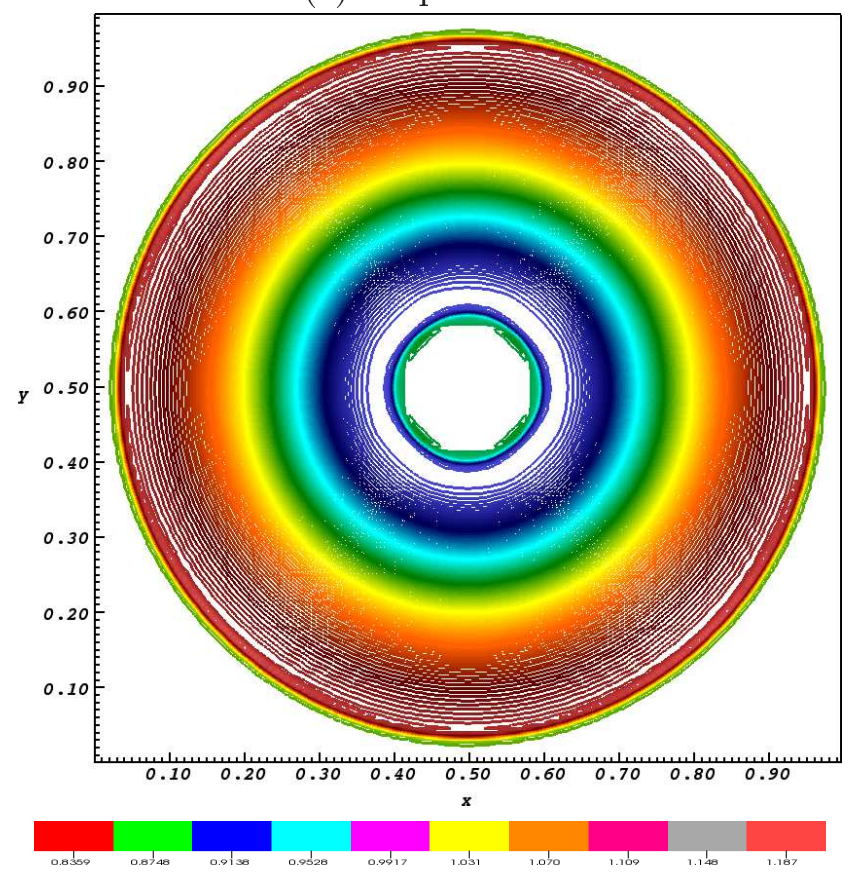

(b) EPUS

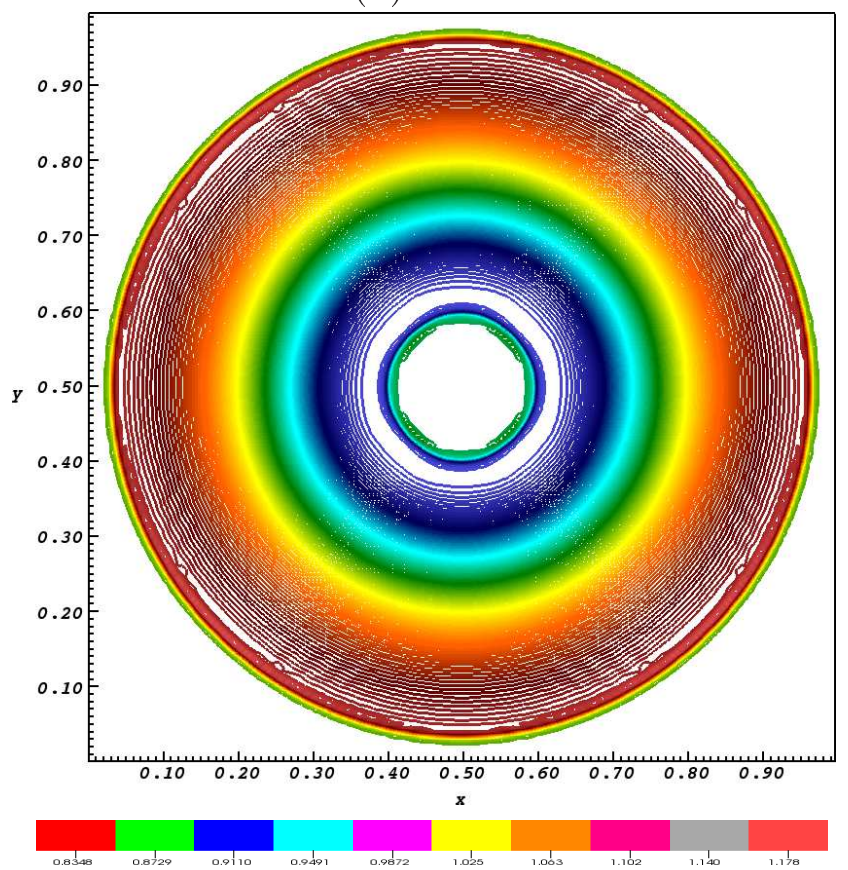

(d) Esquema 2

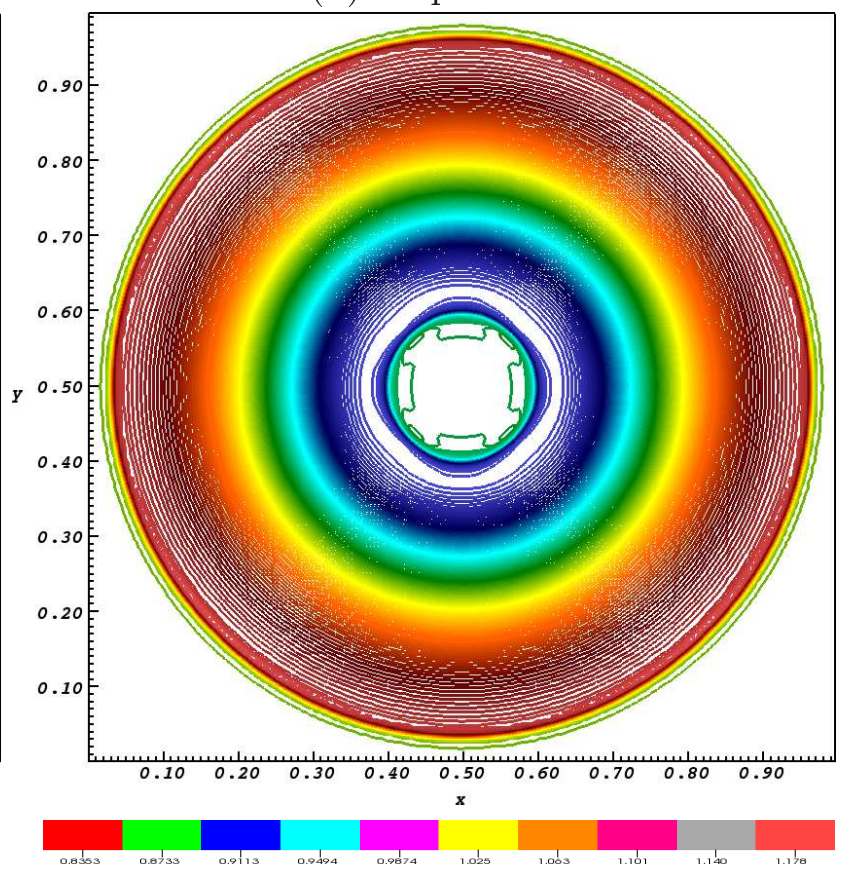

Figura 6.46: Perfil para a altura $h$ do fluido no Teste 10. (a) van Albada; (b) EPUS; (c)-(h) Esquemas 1-6 com os melhores parâmetros. 
(e) Esquema 3

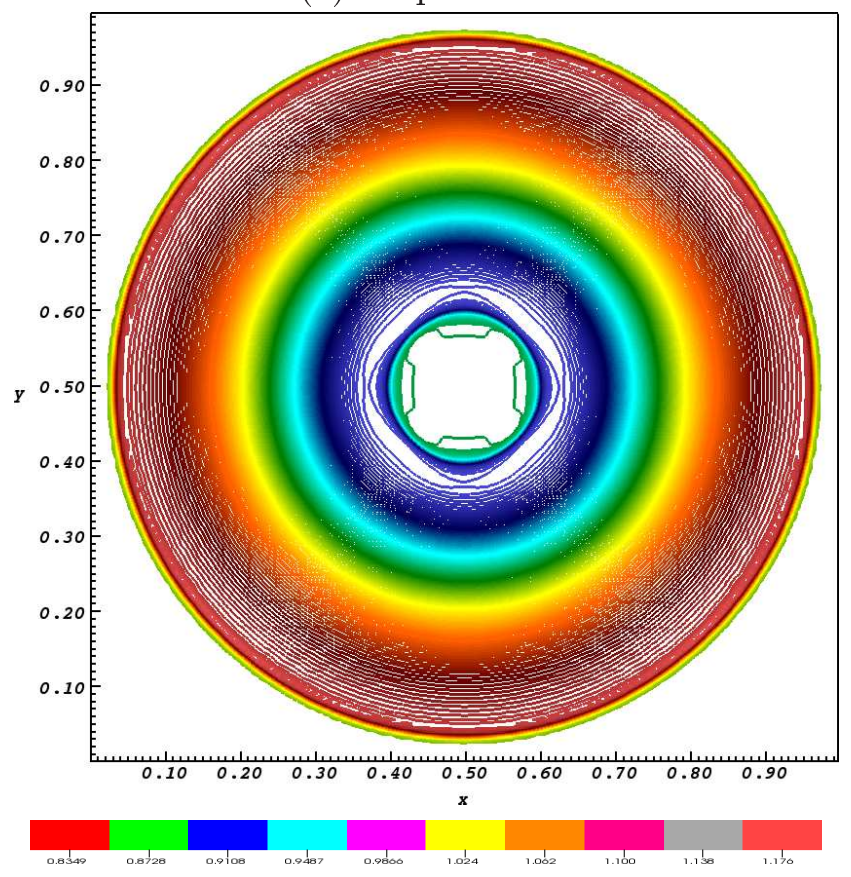

(g) Esquema 5

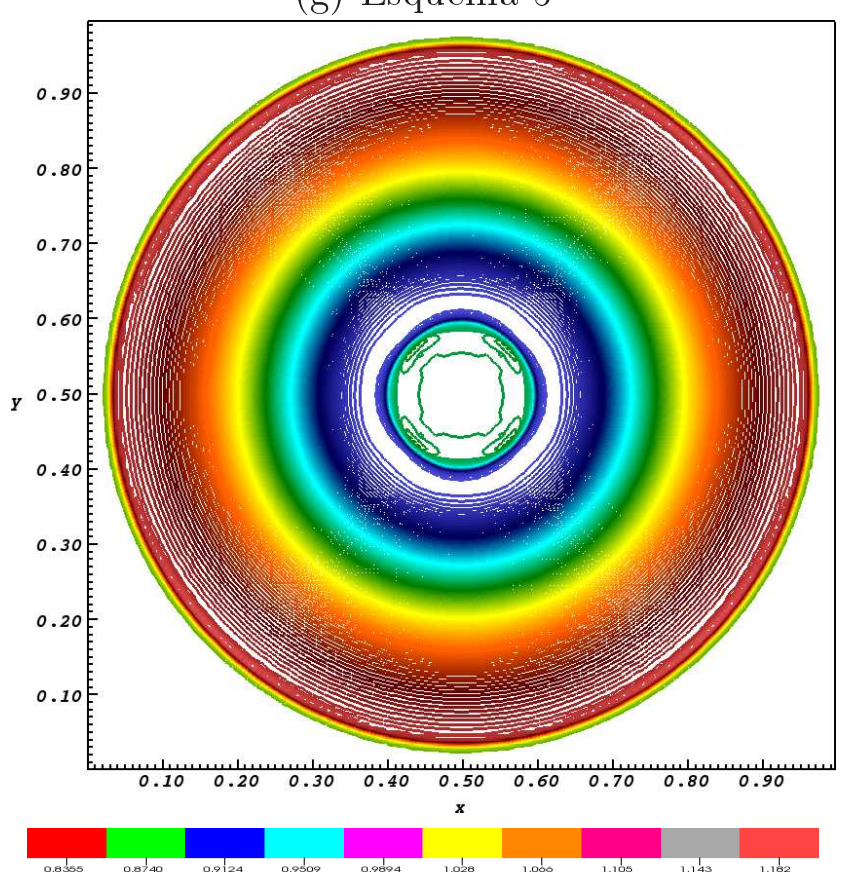

(f) Esquema 4
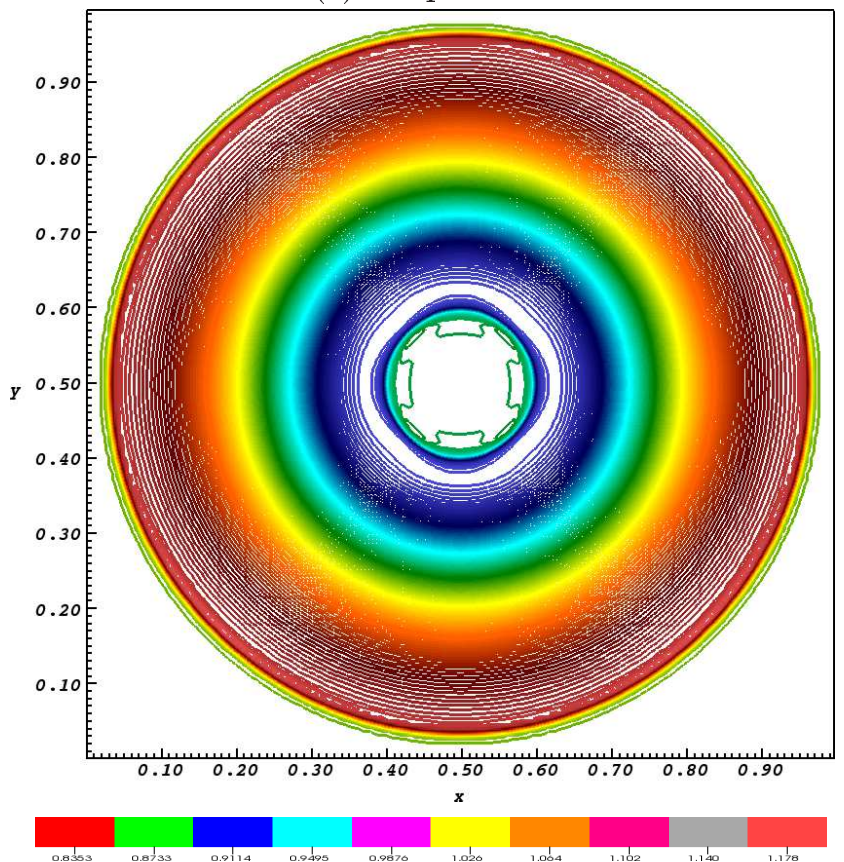

(h) Esquema 6

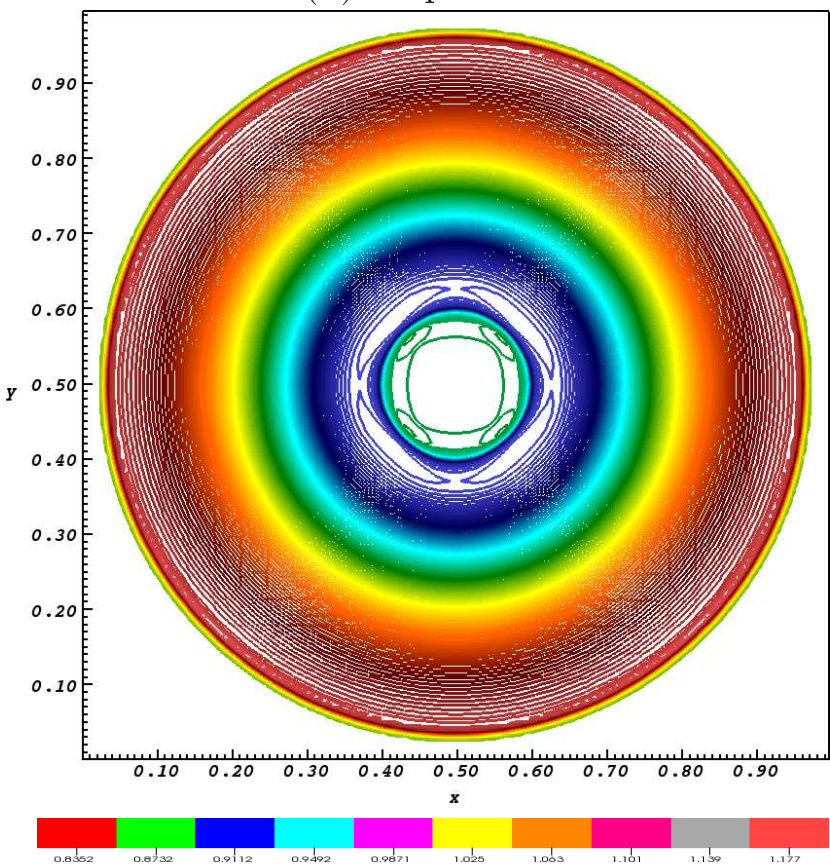

Figura 6.47: Continuação da Figura 6.46. 
Este problema, definido em $[0,2] \times[0,2]$, apresenta a seguinte solução analítica para a variável conservada $\rho$ (ver [32]):

$$
\rho(x, y, t)=1+0.2 \sin (\pi[x+y-(u+v) t]) .
$$

Para simulação foi considerada uma uma malha de $N=320$ pontos, a $C F L=0.5$ e tempo final $T=2$. Os resultados numéricos ao longo da reta $x=y$, obtidos com os esquemas FUS-RF utilizando $\beta=0.1545,0.1605,0.1670,0.1750$ e $\epsilon=0,0.1,0.01,0.001$, e a solução analítica ao longo dessa mesma reta são apresentados na Figura 6.48. A Figura 6.49 apresenta uma comparação dos esquemas FUS-RF, com os melhores valores dos parâmetros $\beta$ e $\epsilon(\beta=0.1545$ para o Esquema 1, $\epsilon=0.1$ para os Esquemas 2, 3, 4 ,6 e $\epsilon=0$ para o Esquema 5), com os resultados dos esquema EPUS e van Albada e com a solução analítica. Por essa figura, nota-se que os esquemas EPUS e Esquema 1 (com $\beta=0.1545$ ) proporcionaram os melhores resultados.

Os erros, as ordens de convergência observadas e as constantes dos erros são apresentados nas Tabelas 6.9-6.11 para vários valores de $\beta$ e $\epsilon$. Verifica-se que todos os esquemas atingiram segunda ordem de convergência na norma $L_{2}$, porém nas normas $L_{1}$ e $L_{\infty}$ alcançaram ordem $\approx 1$.

Teste 12: Este teste, conhecido como Four-Shocks Problem (ver [3], [21]), é caracterizado pela formação de quatro ondas de choque no domínio $[0,1] \times[0,1]$. Inicialmente, existem quatro fluidos com propriedades diferentes contidos em quatro quadrantes distintos, tendo o ponto $(0.8,0.8)$ como contato. A condição inicial é tomada como

$$
\left[\rho_{0}, u_{0}, v_{0}, p_{0}\right]^{T}= \begin{cases}{[0.13799,1.20604,1.20604,0.02903]^{T},} & {[0,0.8] \times[0,0.8]} \\ {[0.53225,0,1.20604,0.3]^{T},} & {[0.8,1] \times[0,0.8]} \\ {[1.5,0,0,1.5]^{T},} & {[0.8,1] \times[0.8,1]} \\ {[0.53225,1.20604,0,0.3]^{T},} & {[0,0.8] \times[0.8,1]}\end{cases}
$$

Este teste foi simulado numericamente considerando-se número de Courant $C F L=0.8$, tempo final $T=0.8$ e duas malhas computacionais de tamanhos $200 \times 200$ e $1000 \times 1000$. Como solução de referência utilizou-se o esquema van Albada na malha de $1000 \times 1000$ células. Na primeira malha os esquemas da família FUS-RF foram analisados resolvendo-se este problemas para vários valores dos parâmetros $\beta$ e $\epsilon$. A Figura 6.50 mostra os resultados para a densidade $\rho$ ao longo da reta $x=y$. Observa-se que os Esquemas 2, 3 e 6 apresentaram os melhores resultados para $\epsilon=0.1$; e os Esquemas 4 e 5 não apresentaram mudanças significativas com as variações do parâmetro $\epsilon$. O Esquema 1 apresentou pequena alteração na solução com a variação do parâmetro $\beta$, apresentando melhor resultado com $\beta=0.1545$. Nas Figuras 6.51 e 6.52, tem-se as comparações entre os esquemas e a solução de referência. Na região de Zoom (6.52) vê-se que o Esquema 1 forneceu resultados semelhantes aos do Esquema EPUS, 
Capítulo 6

Resultados numéricos para leis de conservação hiperbólicas

(a) Esquema 1

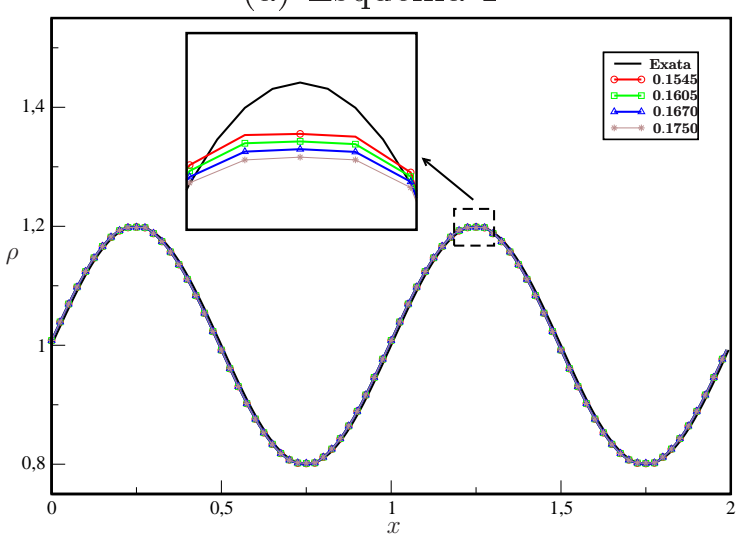

(c) Esquema 3

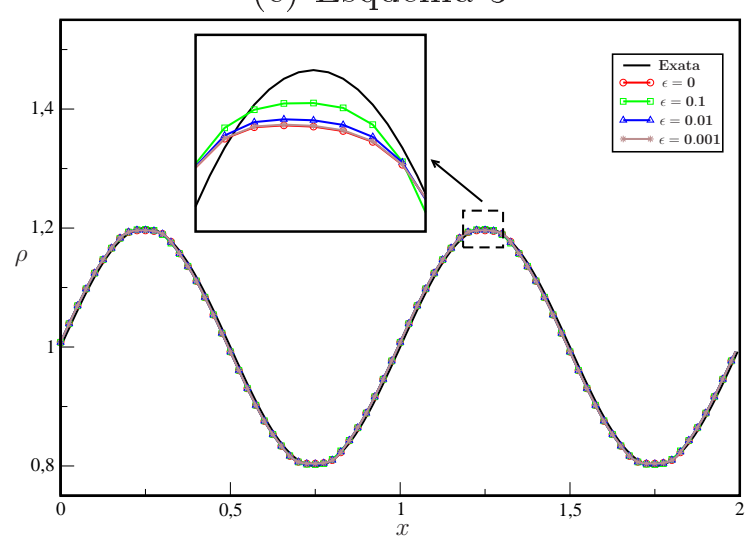

(e) Esquema 5

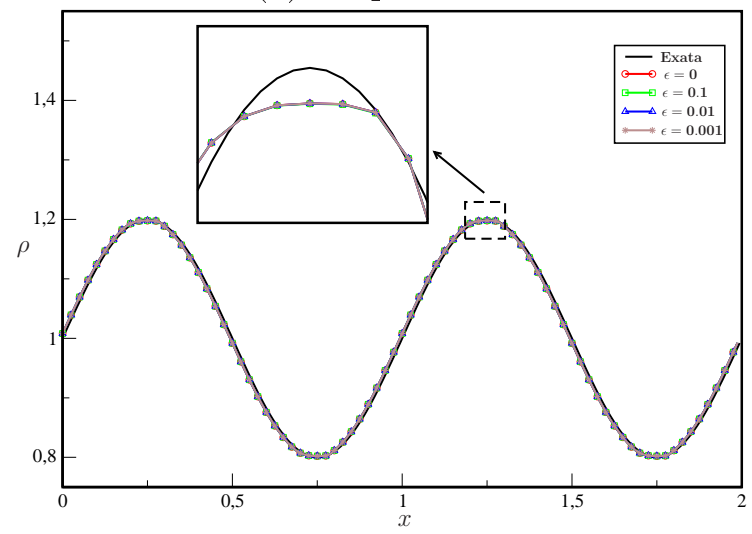

(b) Esquema 2

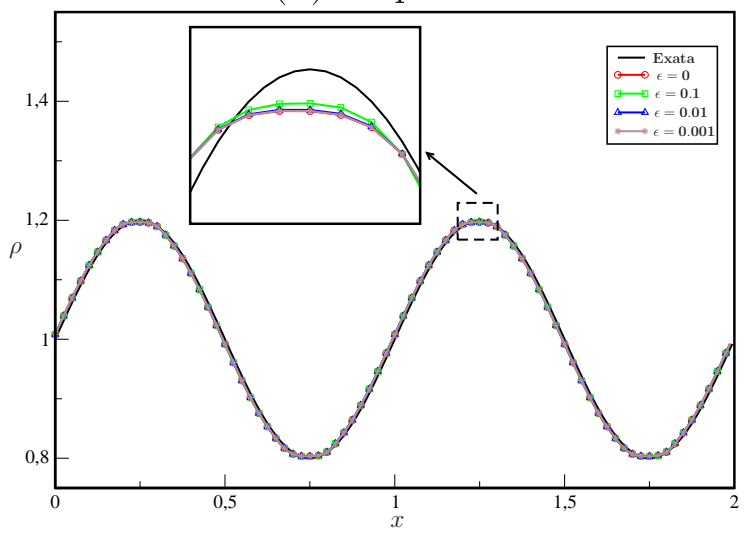

(d) Esquema 4

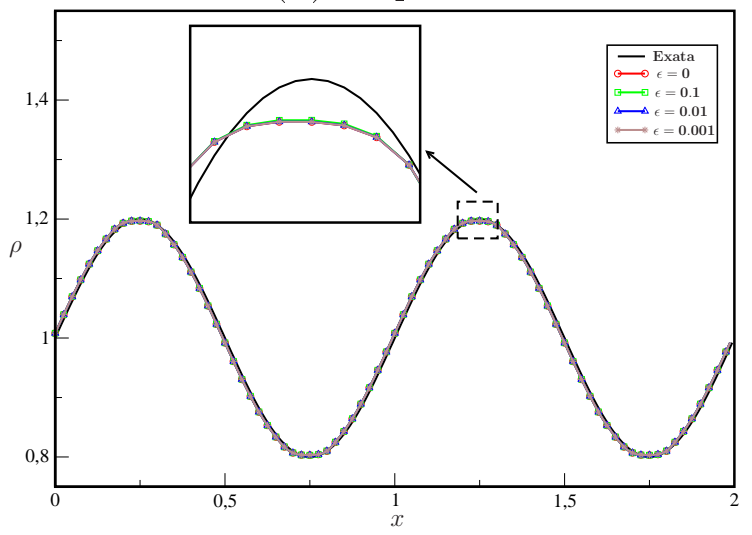

(f) Esquema 6

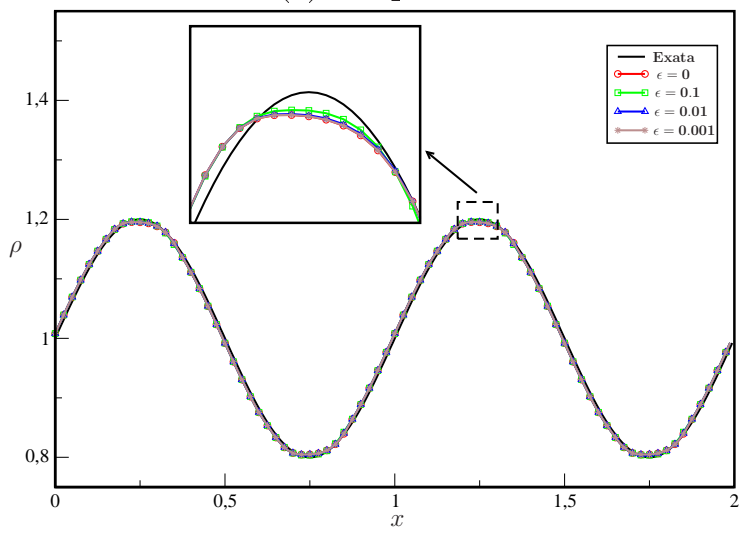

Figura 6.48: Solução exata e resultados obtidos com os esquemas FUS-RF para as equações de Euler - Teste 11. (a) Esquema 1 utilizando vários valores do parâmetro $\beta$; (b)-(f) Esquemas $2-6$ utilizando vários valores do parâmetro $\epsilon$.

90 


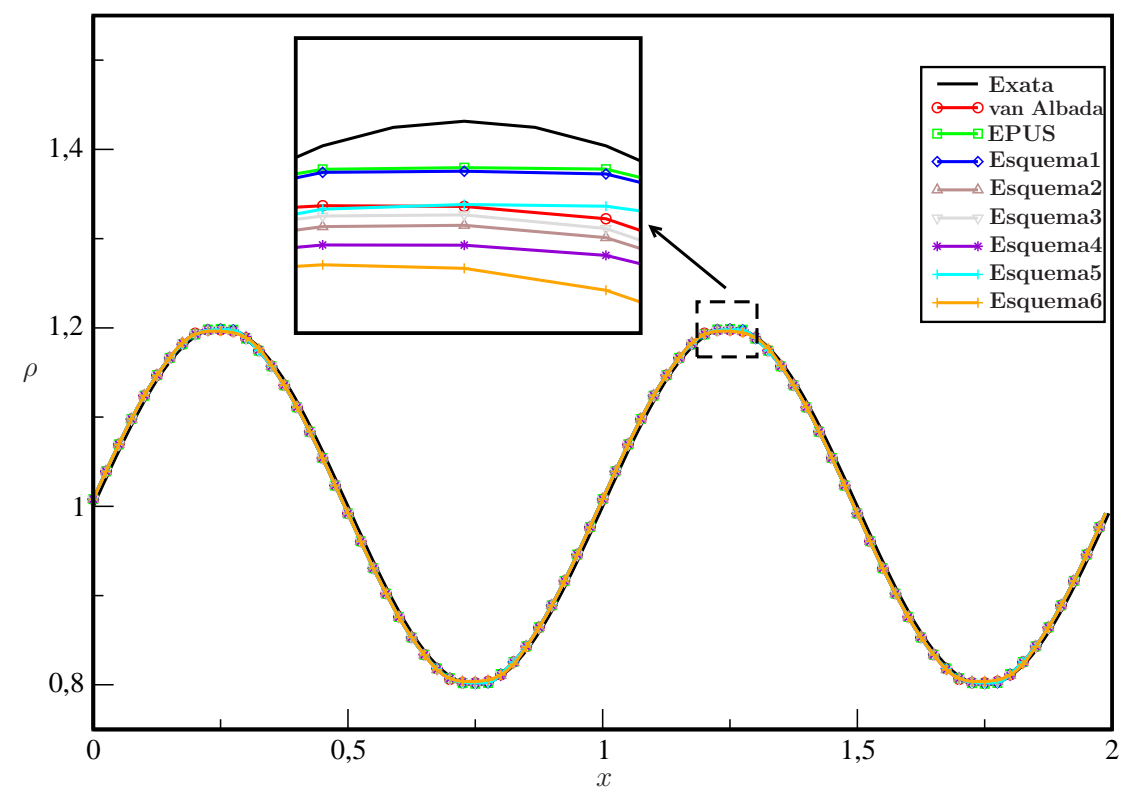

Figura 6.49: Comparação entre a solução exata e as soluções numéricos (Esquemas 1-6 com os melhores parâmetros e referências van Albada e EPUS) para as equações de Euler - Teste 11, mostrando região de ampliação.

Tabela 6.9: Comparação dos erros e ordens observadas nas normas $L_{1}, L_{2}$ e $L_{\infty}$ para o Teste 11, usando os esquemas van Albada, EPUS e os Esquemas 1-6 com vários parâmetros.

\begin{tabular}{l|r|c|c|c|c|c|c|c|c|c}
\hline \hline & $N$ & \multicolumn{3}{|c|}{$L_{1}$} & \multicolumn{4}{c}{$L_{2}$} & \multicolumn{3}{c}{$L_{\infty}$} \\
\cline { 3 - 11 } & & Erro & $q$ & $\mathrm{c}$ & Erro & $q$ & $\mathrm{c}$ & Erro & $q$ & $\mathrm{c}$ \\
\hline \hline Van & 20 & 0.0443643 & - & - & 0.0022410 & - & - & 0.0660403 & - & - \\
Albada & 40 & 0.0211463 & 1.0689 & 0.52 & 0.0005238 & 2.0968 & 0.28 & 0.0277880 & 1.2488 & 1.17 \\
& 80 & 0.0103430 & 1.0317 & 0.46 & 0.0001243 & 2.0742 & 0.26 & 0.0130323 & 1.0923 & 0.73 \\
& 160 & 0.0050784 & 1.0262 & 0.45 & 0.0000304 & 2.0284 & 0.22 & 0.0065402 & 0.9946 & 0.51 \\
& 320 & 0.0025210 & 1.0103 & 0.42 & 0.0000075 & 2.0092 & 0.20 & 0.0032889 & 0.9917 & 0.50 \\
\hline EPUS & 20 & 0.0435657 & - & - & 0.0022979 & - & - & 0.0645151 & - & - \\
& 40 & 0.0204569 & 1.0906 & 0.53 & 0.0005424 & 2.0827 & 0.27 & 0.0282908 & 1.1893 & 0.99 \\
& 80 & 0.0101978 & 1.0043 & 0.41 & 0.0001298 & 2.0622 & 0.26 & 0.0136799 & 1.0482 & 0.65 \\
& 160 & 0.0050673 & 1.0089 & 0.42 & 0.0000315 & 2.0430 & 0.24 & 0.0067152 & 1.0265 & 0.60 \\
& 320 & 0.0025172 & 1.0093 & 0.42 & 0.0000077 & 2.0275 & 0.22 & 0.0033326 & 1.0107 & 0.56 \\
\hline Esq 1 & 20 & 0.0427953 & - & - & 0.0021678 & - & - & 0.0620287 & - & - \\
$\beta=0.1545$ & 40 & 0.0202905 & 1.0766 & 0.51 & 0.0005329 & 2.0241 & 0.22 & 0.0281339 & 1.1406 & 0.85 \\
& 80 & 0.0101612 & 0.9977 & 0.40 & 0.0001295 & 2.0411 & 0.24 & 0.0136893 & 1.0392 & 0.63 \\
& 160 & 0.0050594 & 1.0060 & 0.41 & 0.0000315 & 2.0391 & 0.23 & 0.0067145 & 1.0276 & 0.60 \\
& 320 & 0.0025187 & 1.0062 & 0.41 & 0.0000077 & 2.0268 & 0.22 & 0.0033326 & 1.0106 & 0.56 \\
\hline Esq 1 & 20 & 0.0431095 & - & - & 0.0021906 & - & - & 0.0638671 & - & - \\
$\beta=0.1605$ & 40 & 0.0205426 & 1.0693 & 0.50 & 0.0005382 & 2.0250 & 0.23 & 0.0279436 & 1.1925 & 0.99 \\
& 80 & 0.0102207 & 1.0071 & 0.41 & 0.0001300 & 2.0490 & 0.24 & 0.0136819 & 1.0302 & 0.61 \\
& 160 & 0.0050693 & 1.0116 & 0.42 & 0.0000315 & 2.0432 & 0.24 & 0.0067137 & 1.0270 & 0.60 \\
& 320 & 0.0025226 & 1.0068 & 0.41 & 0.0000077 & 2.0282 & 0.22 & 0.0033326 & 1.0104 & 0.56 \\
\hline \hline Esq 1 & 20 & 0.0434917 & - & - & 0.0022241 & - & - & 0.0656693 & - & - \\
$\beta=0.1670$ & 40 & 0.0208109 & 1.0562 & 0.51 & 0.0005452 & 2.0330 & 0.21 & 0.0277824 & 1.1832 & 0.99 \\
& 80 & 0.0102871 & 1.0125 & 0.41 & 0.0001307 & 2.0472 & 0.22 & 0.0136812 & 1.0386 & 0.61 \\
& 160 & 0.0050781 & 1.0075 & 0.43 & 0.0000316 & 2.0158 & 0.24 & 0.0067145 & 1.0269 & 0.60 \\
& 320 & 0.0025273 & 1.0062 & 0.42 & 0.0000077 & 2.0223 & 0.22 & 0.0033326 & 1.0112 & 0.52 \\
\hline \hline
\end{tabular}


Tabela 6.10: Continuação da Tabela 6.9.

\begin{tabular}{|c|c|c|c|c|c|c|c|c|c|c|}
\hline & \multirow[t]{2}{*}{$\bar{N}$} & \multicolumn{3}{|c|}{$L_{1}$} & \multicolumn{3}{|c|}{$L_{2}$} & \multicolumn{3}{|c|}{$L_{\infty}$} \\
\hline & & Erro & $q$ & $\mathrm{c}$ & Erro & $q$ & $\mathrm{c}$ & Erro & $q$ & $\mathrm{c}$ \\
\hline Esq 2 & 20 & 0.0467928 & $=$ & & 0.0023814 & - & & 0.0608023 & & \\
\hline \multirow[t]{4}{*}{$\epsilon=0$} & 40 & 0.0222070 & 1.0752 & 0.55 & 0.0005841 & 2.0273 & 0.25 & 0.0333697 & 0.8655 & 0.44 \\
\hline & 80 & 0.0106097 & 1.0656 & & 0.0001351 & 2.1115 & 0.32 & 0.0141536 & 1.2373 & 1.35 \\
\hline & 160 & 0.0051927 & 1.0308 & 0.47 & 0.0000321 & 2.0728 & 0.28 & 0.0067274 & 1.0730 & 0.74 \\
\hline & 320 & 0.0025546 & 1.0233 & 0.46 & 0.0000077 & 2.0427 & 0.24 & 0.0033321 & 1.0135 & 0.57 \\
\hline \multirow{5}{*}{$\begin{array}{l}\text { Esq 2 } \\
\epsilon=0.1\end{array}$} & 20 & 0.0469260 & - & - & 0.0024906 & - & & 0.0663234 & - & \\
\hline & 40 & 0.0221675 & 1.0819 & 0.56 & 0.0005859 & 2.0877 & 0.30 & 0.0325762 & 1.0256 & 0.70 \\
\hline & 80 & 0.0106242 & 1.0610 & 0.53 & 0.0001339 & 2.1286 & 0.34 & 0.0139190 & 1.2267 & 1.28 \\
\hline & 160 & 0051811 & 1.0360 & & 0.0000318 & 2.0723 & 0.27 & .0066 & 1.0555 & 0.68 \\
\hline & 320 & 0.0025472 & 1.0243 & 0.46 & 0.0000077 & 2.0380 & 0.24 & 0.0033 & 1.0097 & 0.55 \\
\hline \multirow{5}{*}{$\begin{array}{l}\text { Esq 2 } \\
\epsilon=0.01\end{array}$} & 20 & 0.0468146 & & - & 0.0023939 & & & 0.0618 & & \\
\hline & 40 & .0222040 & 1.0761 & 0.55 & 0.0005 & 2.0352 & 0.25 & .0332 & 0.8966 & 0.48 \\
\hline & 80 & 0.0106136 & 1.0649 & 0.53 & 0.00013 & 2.1133 & 0.32 & .0141 & 1.2351 & 1.34 \\
\hline & 160 & 0.0051913 & 1.0317 & 0.47 & 0.0000320 & 2.0726 & 0.28 & 229 & 1.0707 & 0.73 \\
\hline & 320 & 0.0025533 & 1.0237 & 0.46 & 0.0000077 & 2.0420 & 0.24 & .003 & 1.0129 & 0.56 \\
\hline Esq 3 & 20 & & 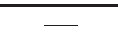 & & & & & & & \\
\hline \multirow[t]{4}{*}{$\epsilon=0$} & 40 & & 1.0737 & & & 2.0180 & 0.24 & & .7188 & 0.29 \\
\hline & 80 & 0.0106 & 1.0605 & 0.53 & 0.00 & 2.1128 & 0.3 & 74 & 1.2728 & 1.55 \\
\hline & 160 & 0.0052117 & 1.0287 & 0.47 & 0.0000 & 2.0773 & 0.29 & 90 & 1.0761 & 0.75 \\
\hline & & 0.00257 & 1.0193 & 0.45 & 0.0000 & 2.0495 & 0.25 & .003 & .0139 & 0.57 \\
\hline Esq 3 & 20 & 0.0470041 & & & 0.0025399 & & & 49 & & \\
\hline \multirow[t]{4}{*}{$\epsilon=0.1$} & 40 & & 1.0928 & & & 2.1355 & 0.34 & & 1.1060 & 0.86 \\
\hline & 80 & & & & & & & & & 1.21 \\
\hline & 160 & 0. & 1.0376 & 0. & 0.00 & 2.0662 & 0.2 & & 1.0320 & 0.61 \\
\hline & 320 & 0.002 & 1.0213 & 0.45 & 0.000 & 2.0315 & 0.23 & 5 & 1.0041 & 0.54 \\
\hline \multirow{5}{*}{$\begin{array}{l}\text { Esq 3 } \\
\epsilon=0.01\end{array}$} & 20 & & & & & & & & & \\
\hline & 40 & 0.02223 & 1.0904 & & & 2.0407 & 0.26 & & 0.6838 & 0.26 \\
\hline & 80 & & 1.0701 & & & & 0.3 & & 1.2585 & 1.45 \\
\hline & 160 & & & & & & & & & 0.71 \\
\hline & 320 & & 1.0191 & 0.4 & & 2.0438 & 0.2 & & .0121 & 0.56 \\
\hline \multirow{5}{*}{$\begin{array}{l}\text { Esq } 4 \\
\epsilon=0\end{array}$} & 20 & 0 . & & 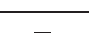 & 0.00 & & & & & \\
\hline & 40 & 0.02 & 1.0748 & 0.55 & 44 & 2.0252 & 0.25 & & 0.8896 & 0.47 \\
\hline & 80 & & & & & 2.11 & & & & 1.35 \\
\hline & 160 & & & & 0.000 & & 0.2 & & 1.0679 & 0.72 \\
\hline & 320 & 0.00255 & 1.0220 & 0.45 & 0.0000 & 2.0432 & 0.24 & 22 & 1.0130 & 0.56 \\
\hline \multirow{5}{*}{$\begin{array}{l}\text { Esq 4 } \\
\epsilon=0.1\end{array}$} & 20 & & & & & & - & & & \\
\hline & 40 & & & & & 2.036 & ת 0 & & & 0.53 \\
\hline & 80 & & 0638 & & & 2.1134 & 0.3 & & 1.2348 & 1.33 \\
\hline & 160 & 0.0051 & 1.0323 & & 0.000 & 2.0740 & 0.2 & 84 & 1.0647 & 0.71 \\
\hline & 320 & 0.00255 & 1.0228 & 0.45 & 0.0000 & 2.0420 & 0.24 & 0.0033 & 1.0120 & 0.56 \\
\hline \multirow{5}{*}{$\begin{array}{l}\text { Esq 4 } \\
\epsilon=0.01\end{array}$} & 20 & 0.0467041 & - & - & $0.002:$ & - & - & & & - \\
\hline & 40 & & & & & & 0.26 & & & 0.58 \\
\hline & 80 & & & & & & & & & 1.63 \\
\hline & 160 & & & 0.4 & & & 0.2 & & & 0.75 \\
\hline & 320 & & 1.0221 & 0.46 & & 2.0421 & 0.25 & 0.003 & 1.0130 & 0.58 \\
\hline \multirow{5}{*}{$\begin{array}{l}\text { Esq } 5 \\
\epsilon=0\end{array}$} & 20 & 0.04575 & - & - & $\overline{25}$ & - & - & & & \\
\hline & 40 & 0.0217258 & 1.0743 & 0.54 & 0.0005 & 2.0394 & 0.2 & 030907 & 1.1601 & 0.99 \\
\hline & 80 & 0.0105195 & 1.0463 & 0.49 & 0.0001374 & 2.1059 & 0.3 & 0.014308 & 1.1110 & 0.86 \\
\hline & 160 & 0.00515 & 1.028 & 0.4 & 0.000 & & 0.2 & & 1.0707 & 0.74 \\
\hline & 320 & 0.0025490 & 1.0163 & 0.44 & 0.0000078 & 2.0488 & 0.25 & 0.003353 & 1.0223 & 0.60 \\
\hline
\end{tabular}


Tabela 6.11: Continuação da Tabela 6.10.

\begin{tabular}{l|r|c|c|c|c|c|c|c|c|c}
\hline \hline & $N$ & \multicolumn{3}{|c|}{$L_{1}$} & \multicolumn{4}{c|}{$L_{2}$} & \multicolumn{4}{c}{$L_{\infty}$} \\
\cline { 3 - 11 } & & Erro & $q$ & $\mathrm{c}$ & Erro & $q$ & $\mathrm{c}$ & Erro & $q$ & $\mathrm{c}$ \\
\hline \hline Esq 5 & 20 & 0.0456892 & - & - & 0.0024215 & - & - & 0.0689677 & - & - \\
$\epsilon=0.1$ & 40 & 0.0217180 & 1.0729 & 0.54 & 0.0005903 & 2.0363 & 0.26 & 0.0308959 & 1.1585 & 0.99 \\
& 80 & 0.0105188 & 1.0459 & 0.49 & 0.0001372 & 2.1050 & 0.32 & 0.0142841 & 1.1129 & 0.86 \\
& 160 & 0.0051561 & 1.0286 & 0.46 & 0.0000324 & 2.0809 & 0.29 & 0.0068081 & 1.0690 & 0.73 \\
& 320 & 0.0025487 & 1.0164 & 0.44 & 0.0000078 & 2.0482 & 0.25 & 0.0033524 & 1.0220 & 0.59 \\
\hline Esq 5 & 20 & 0.0457437 & - & - & 0.0024313 & - & - & 0.0690591 & - & - \\
$\epsilon=0.01$ & 40 & 0.0217249 & 1.0742 & 0.54 & 0.0005916 & 2.0390 & 0.26 & 0.0309060 & 1.1599 & 0.99 \\
& 80 & 0.0105195 & 1.0462 & 0.49 & 0.0001374 & 2.1058 & 0.32 & 0.0143063 & 1.1112 & 0.86 \\
& 160 & 0.0051563 & 1.0286 & 0.46 & 0.0000324 & 2.0819 & 0.29 & 0.0068118 & 1.0705 & 0.74 \\
& 320 & 0.0025490 & 1.0163 & 0.44 & 0.0000078 & 2.0488 & 0.25 & 0.0033536 & 1.0223 & 0.60 \\
\hline Esq 6 & 20 & 0.0465562 & - & - & 0.0024703 & - & - & 0.0595994 & - & - \\
$\epsilon=0$ & 40 & 0.0221708 & 1.0703 & 0.54 & 0.0005635 & 2.1320 & 0.33 & 0.0316874 & 0.9113 & 0.48 \\
& 80 & 0.0106902 & 1.0523 & 0.51 & 0.0001345 & 2.0659 & 0.27 & 0.0143888 & 1.1389 & 0.96 \\
& 160 & 0.0052073 & 1.0376 & 0.49 & 0.0000324 & 2.0512 & 0.26 & 0.0067275 & 1.0968 & 0.82 \\
& 320 & 0.0025832 & 1.0113 & 0.43 & 0.0000078 & 2.0433 & 0.25 & 0.0033323 & 1.0135 & 0.57 \\
\hline Esq 6 & 20 & 0.0471364 & - & - & 0.0023859 & - & - & 0.0545634 & - & - \\
$\epsilon=0.1$ & 40 & 0.0222525 & 1.0828 & 0.57 & 0.0005708 & 2.0634 & 0.27 & 0.0331910 & 0.7171 & 0.28 \\
& 80 & 0.0106274 & 1.0661 & 0.54 & 0.0001330 & 2.1013 & 0.30 & 0.0140850 & 1.2366 & 1.34 \\
& 160 & 0.0052041 & 1.0300 & 0.47 & 0.0000319 & 2.0583 & 0.26 & 0.0066901 & 1.0740 & 0.74 \\
& 320 & 0.0025569 & 1.0252 & 0.46 & 0.0000077 & 2.0367 & 0.24 & 0.0033241 & 1.0090 & 0.55 \\
\hline Esq 6 & 20 & 0.0466229 & - & - & 0.0024257 & - & - & 0.0576290 & - & - \\
$\epsilon=0.01$ & 40 & 0.0222252 & 1.0688 & 0.54 & 0.0005661 & 2.0992 & 0.30 & 0.0322364 & 0.8381 & 0.39 \\
& 80 & 0.0106993 & 1.0546 & 0.52 & 0.0001350 & 2.0675 & 0.27 & 0.0143463 & 1.1680 & 1.06 \\
& 160 & 0.0052099 & 1.0381 & 0.49 & 0.0000324 & 2.0585 & 0.26 & 0.0067236 & 1.0933 & 0.80 \\
& 320 & 0.0025778 & 1.0150 & 0.44 & 0.0000078 & 2.0445 & 0.25 & 0.0033312 & 1.0131 & 0.56 \\
\hline \hline
\end{tabular}

e ambos apresentaram resultados superiores aos outros esquemas. O Esquema 5 com $\epsilon=0,1$ também forneceu um resultado satisfatório, apresentando melhores resultados que o esquema van Albada.

Nas Figuras 6.53 e 6.54 estão os resultados para a densidade na malha $1000 \times 1000$ células usando os esquemas FUS-RF (selecionando-se os melhores parâmetros das simulações apresentadas na Figura 6.50), EPUS e van Albada. Observa-se que o Esquema 1 forneceu resultados bastantes semelhantes aos do esquema EPUS; sendo este último o que introduziu menos viscosidade artificial (dissipação) na simulação computacional. Nota-se também que o Esquema 5 forneceu mais estruturas vorticais que o esquema van Albada. Os outros esquemas, isto é os Esquemas 2, 3, 4 e 6, introduziram praticamente a mesma viscosidade numérica apresentada pelo van Albada.

Teste 13: Neste teste, que utiliza as equações de Euler 2D com termo fonte, simulam-se as instabilidades de Rayleigh-Taylor. O problema consiste na interação entre dois gases com densidades distintas. Na simulação numérica toma-se o domínio $\left[0, \frac{1}{4}\right] \times[0,1]$ e assumem-se as 
(a) Esquema 1

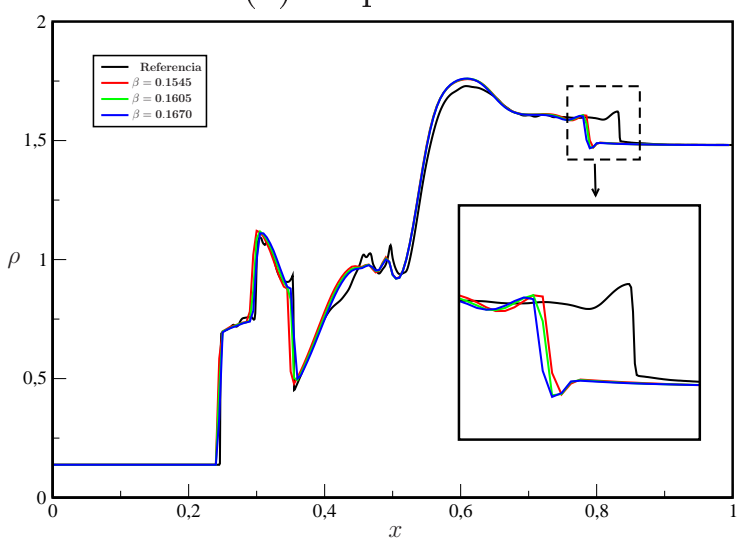

(c) Esquema 3

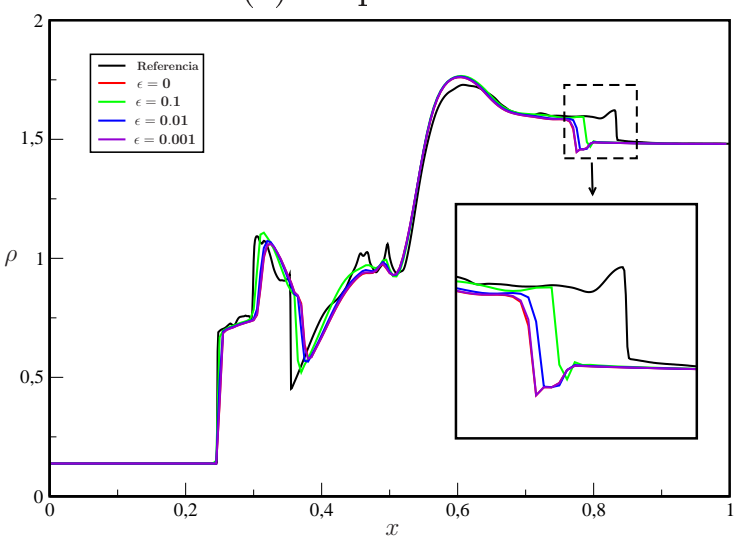

(e) Esquema 5

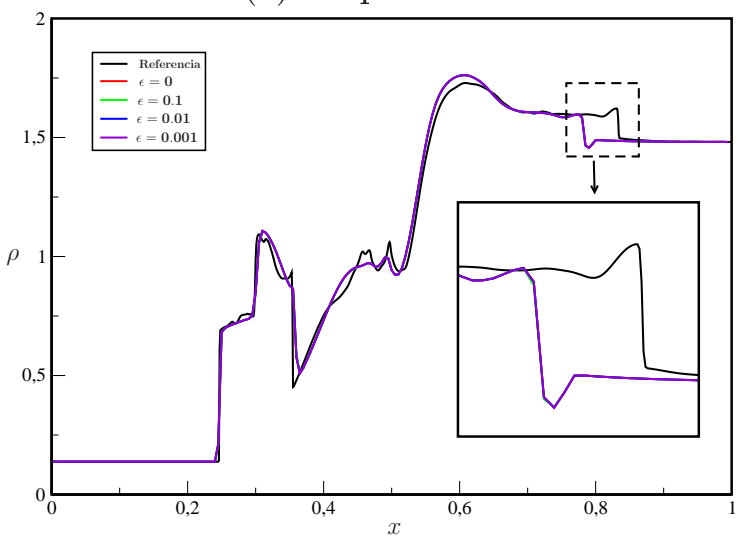

(b) Esquema 2

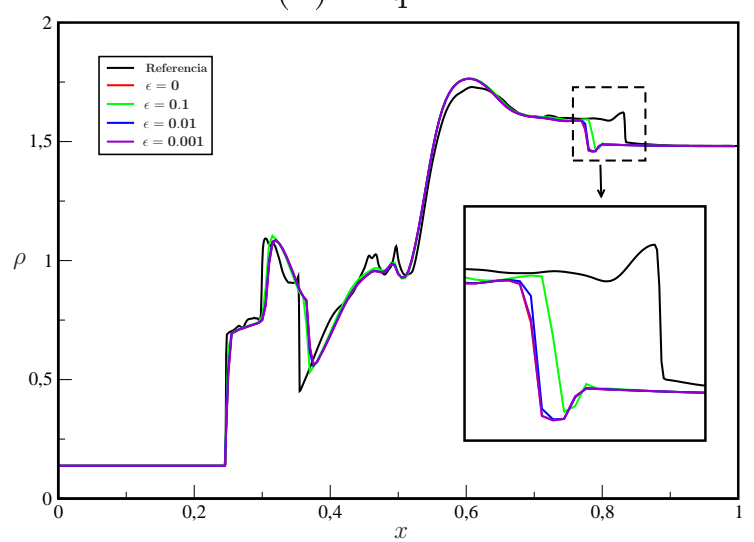

(d) Esquema 4

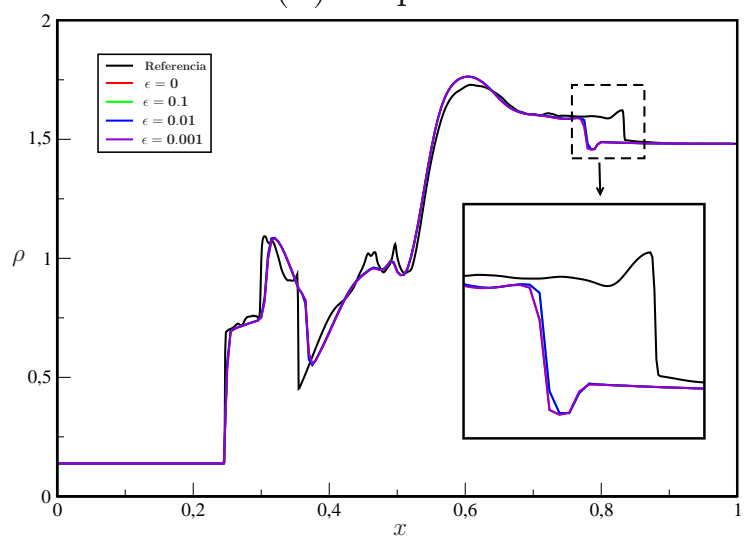

(f) Esquema 6

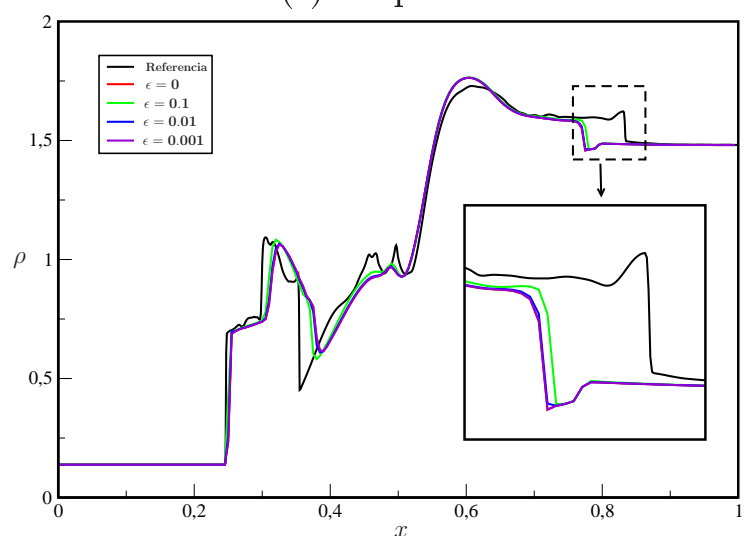

Figura 6.50: Solução de referência e resultados obtidos com os esquemas FUS-RF sob a reta $x=y$ com regiões de ampliação . (a) Esquema 1 utilizando vários valores do parâmetro $\beta$; (b)-(f) Esquemas $2-6$ utilizando vários valores do parâmetro $\epsilon$. 


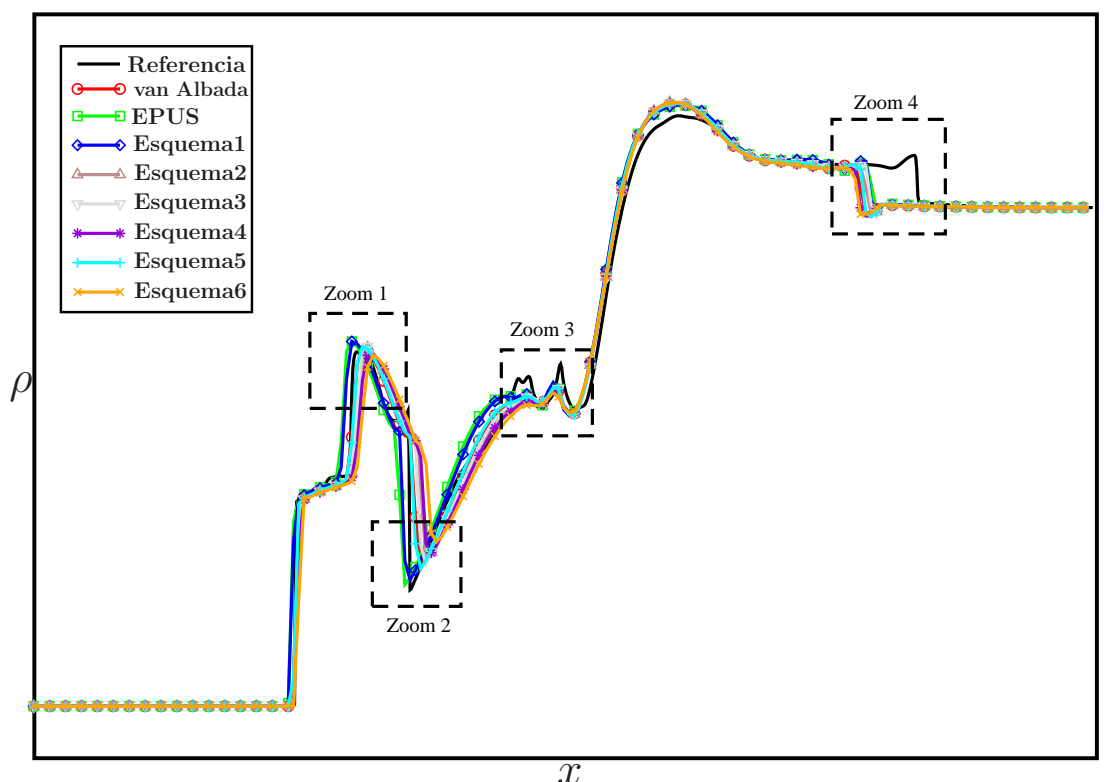

Figura 6.51: Solução de referência e resultados numéricos (Esquemas 1-6 com os melhores parâmetros, van Albada e EPUS) para o Teste 12, mostrando região de ampliação (zoom 1 zoom 4).

(a) Zoom 1

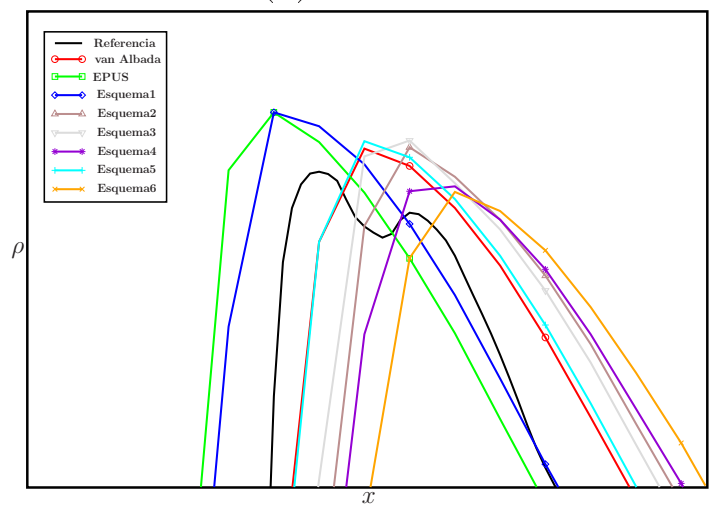

(c) Zoom 3

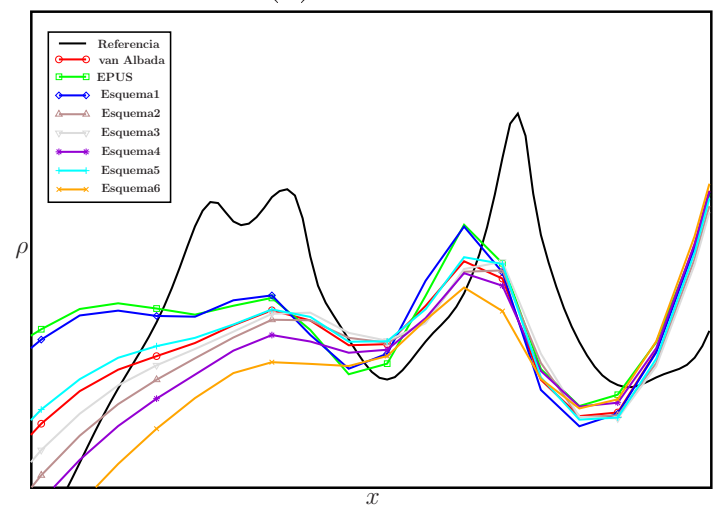

(b) Zoom 2

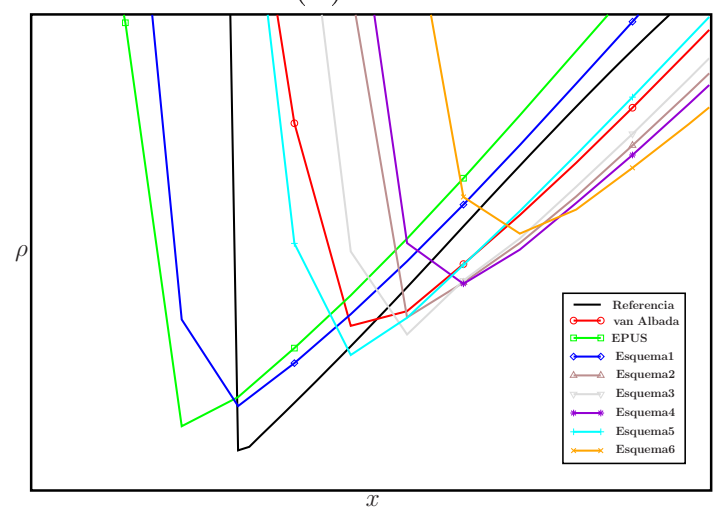

(d) Zoom 4

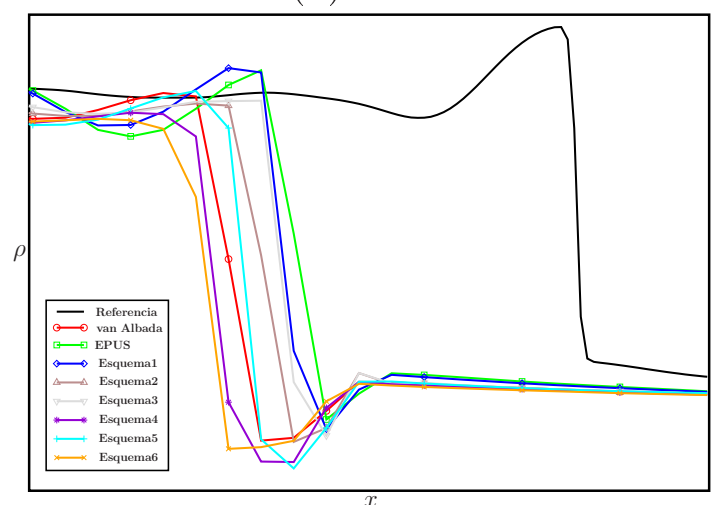

Figura 6.52: Continuação da Figura 6.51. 
(a) van Albada

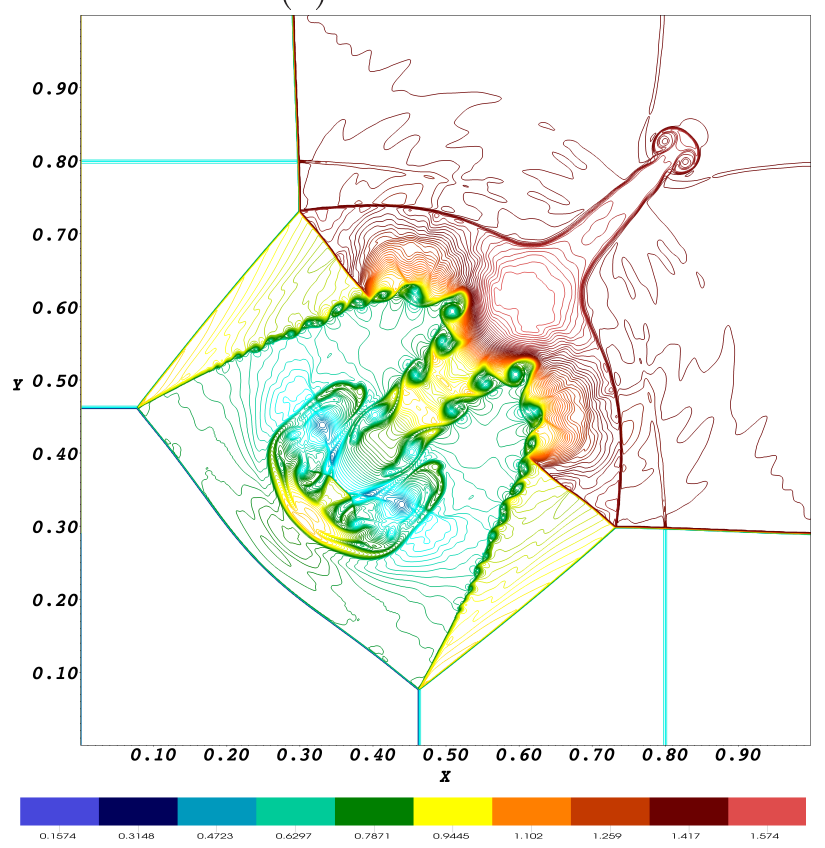

(c) Esquema 1

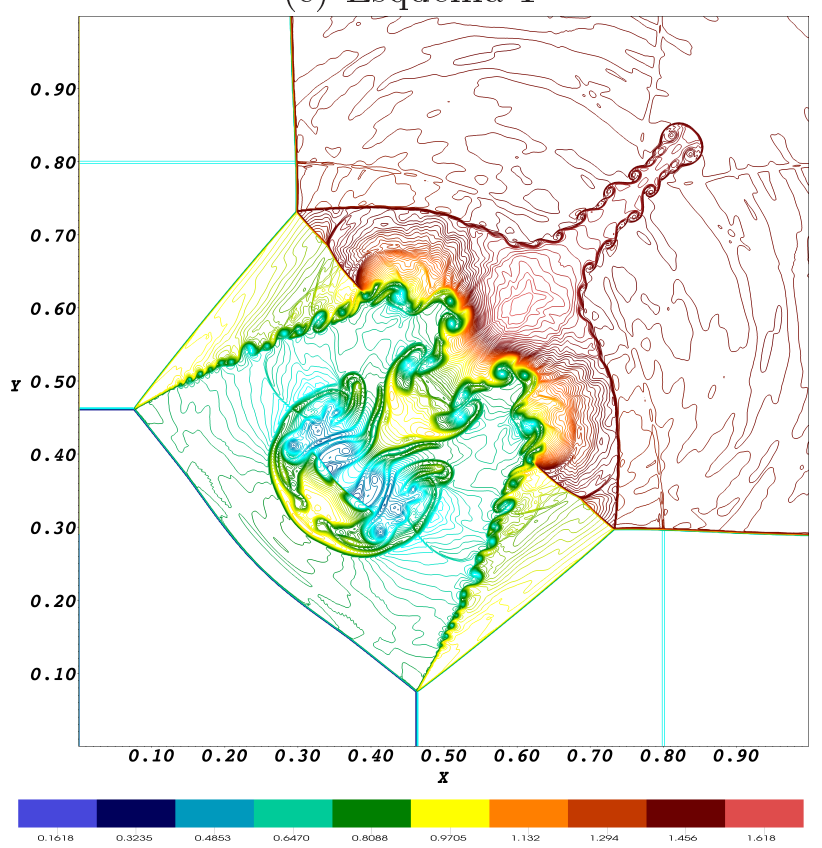

(b) EPUS

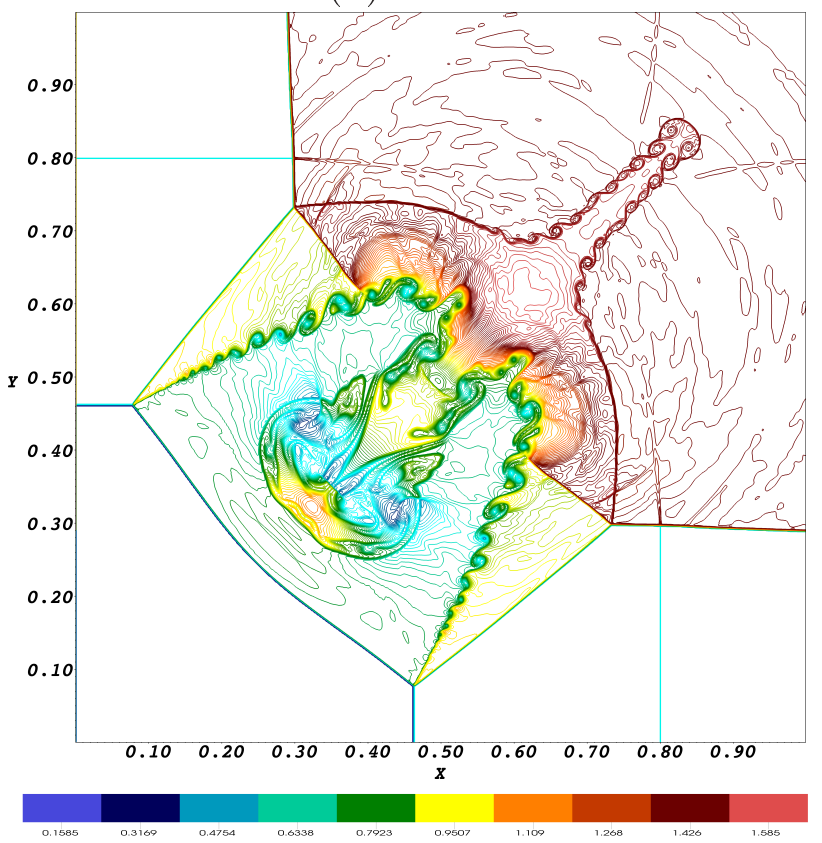

(d) Esquema 2

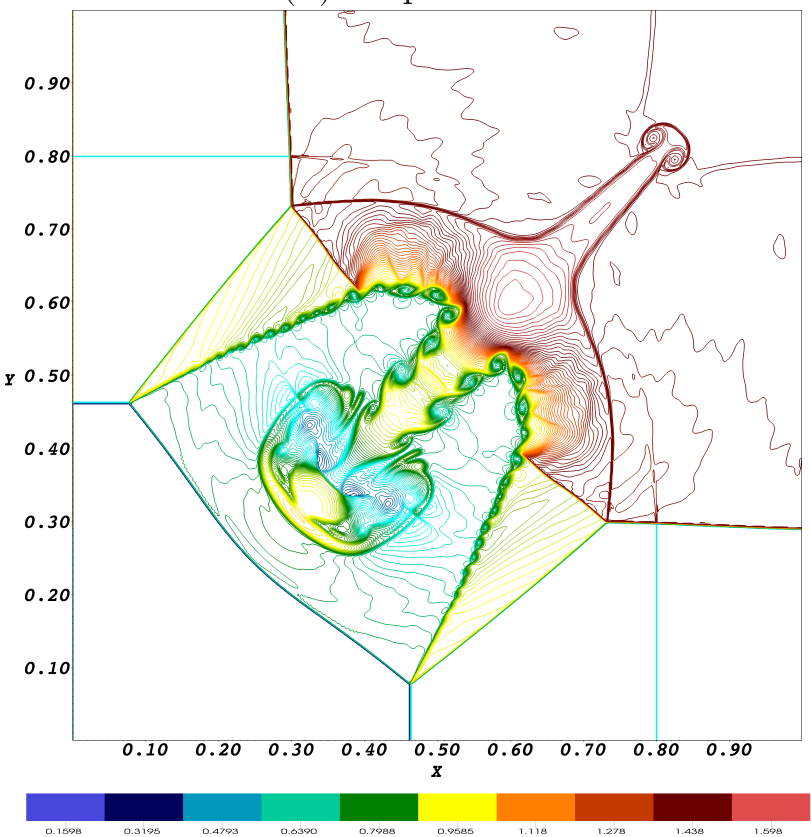

Figura 6.53: Perfil para a densidadde $\rho$ do fluido no Teste 12. (a) van Albada; (b) EPUS; (c)-(h) Esquemas 1-6 com os melhores parâmetros. 
(e) Esquema 3

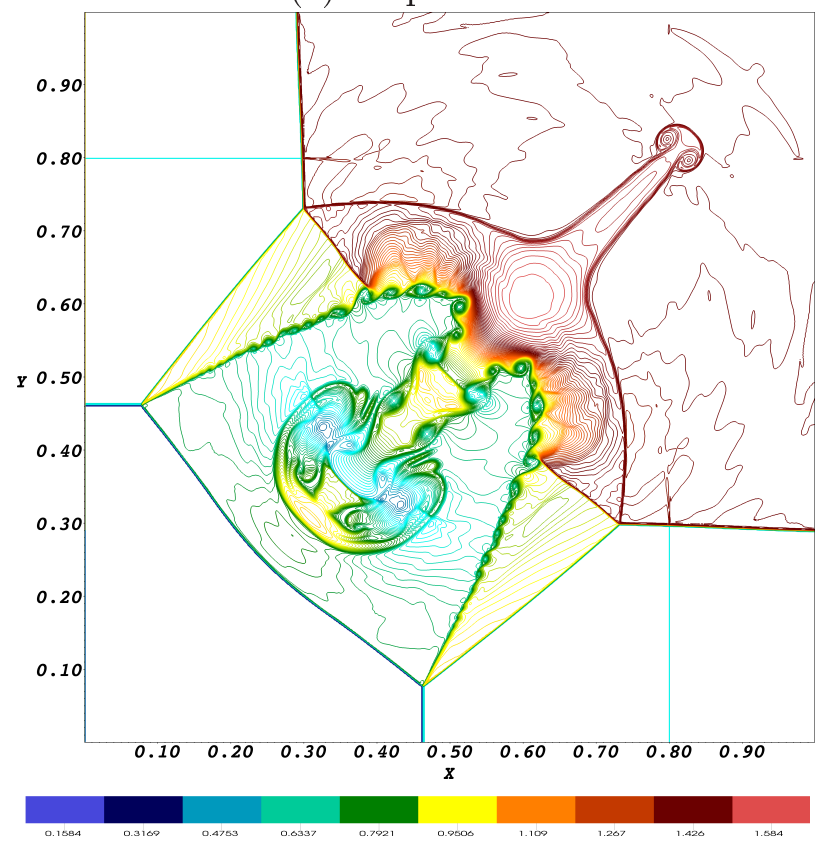

(g) Esquema 5

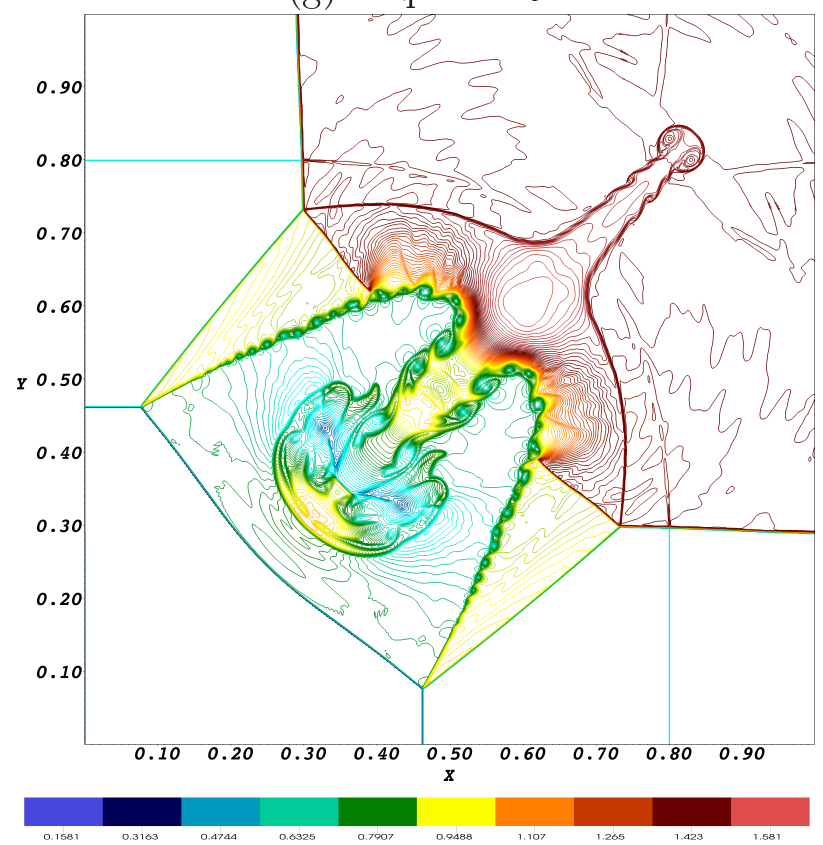

(f) Esquema 4

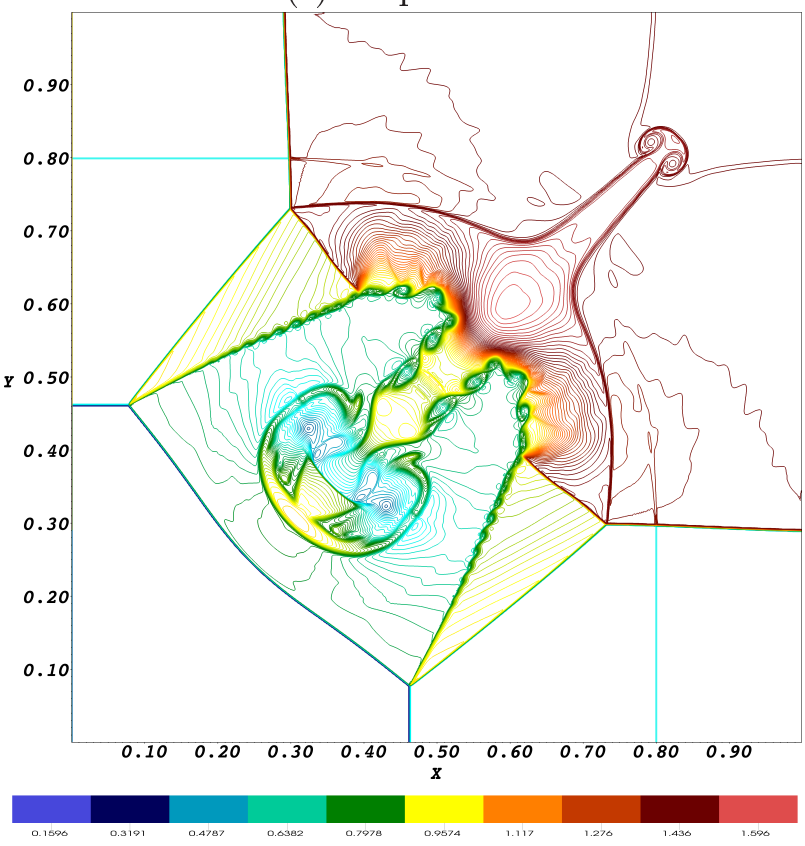

(h) Esquema 6

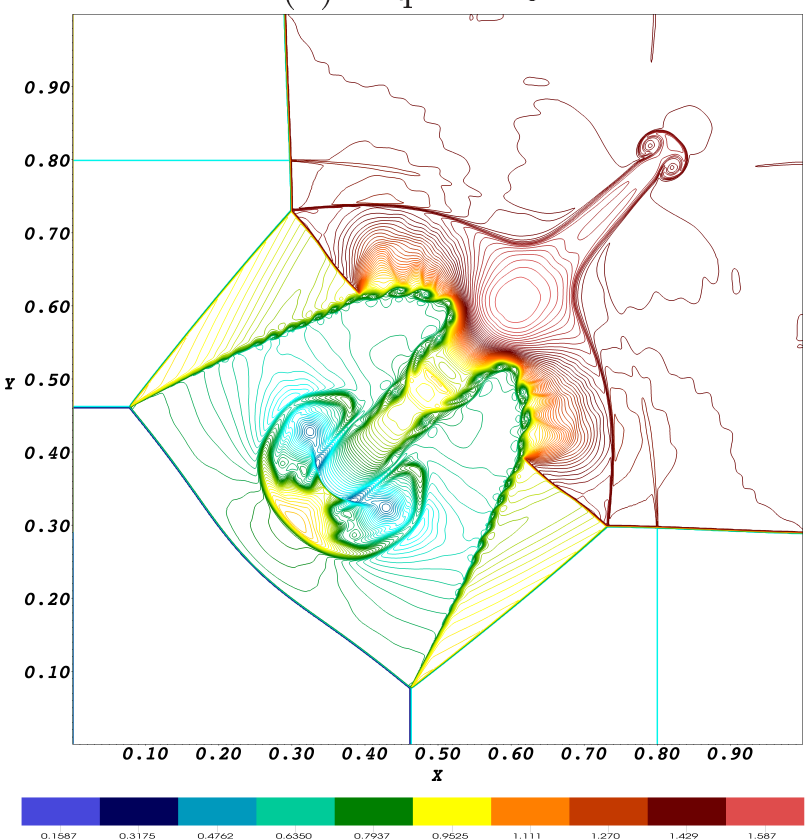

Figura 6.54: Continuação da Figura 6.53. 
seguintes condições iniciais, propostas por Shi et al. [38]

$$
\left[\rho_{0}, u_{0}, v_{0}, p_{0}\right]^{T}= \begin{cases}{[2,0,-0.025 c \cos (8 \pi x), 2 y+1]^{T},} & 0 \leq y<\frac{1}{2} \\ {\left[1,0,-0.025 c \cos (8 \pi x), y+\frac{3}{2}\right]^{T},} & \frac{1}{2} \leq y \leq 1\end{cases}
$$

onde $c=\sqrt{\frac{\gamma p}{\rho}}$ e $\gamma=\frac{5}{3}$. A solução numérica é calculada até o tempo final $T=1.95$, com $C F L=0.6$, nas malhas $240 \times 960$ (grossa) e $480 \times 1920$ (fina) células computacionais. Os resultados obtidos com os esquemas FUS-RF ( $\operatorname{com} \beta=0.1545$ pra o Esquema $1 ; \epsilon=0.1$ para os Esquemas 2, 3 e 6; $\epsilon=0$ para o Esquema 5; e $\epsilon=0.01$ para o Esquema 4), são comparados com os resultados obtidos com os esquemas EPUS, van Albada e WENO5 de quinta ordem (ver Shi et al. [38]). A figura 6.55 contém as soluções computacionais na malha grossa. Como pode ser visto por essa figura (e também da Figura 6.56 que corresponde à malha fina), os resultados proporcionam as mesmas informações daqueles apresentadas no Teste 12 já comentadas anteriormente. Observa-se claramente por essas figuras que os esquemas EPUS e Esquema 1 forneceram resultados bem semelhantes e, surpreendentemente, superiores aos resultados fornecidos pelo WENO5. 
(a) Weno5

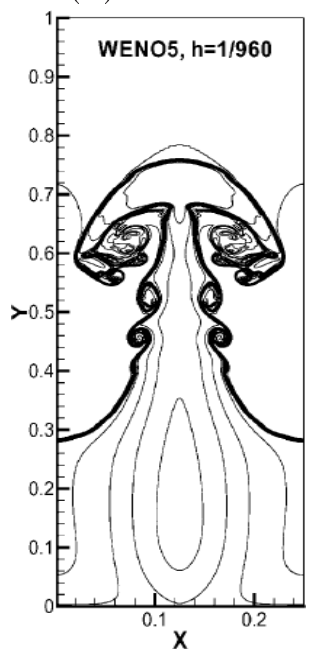

(d) Esquema 1

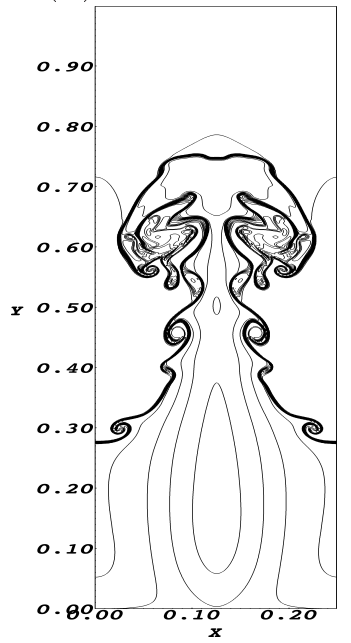

(g) Esquema 4

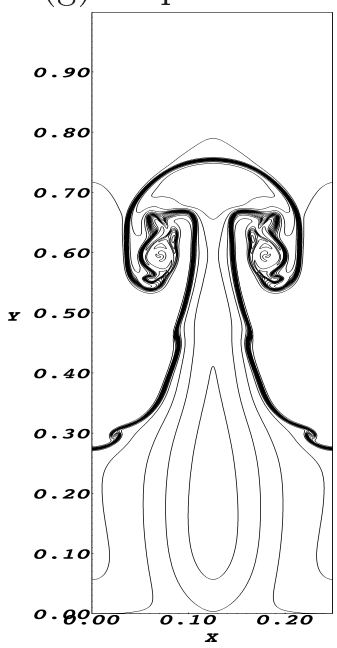

(b) van Albada

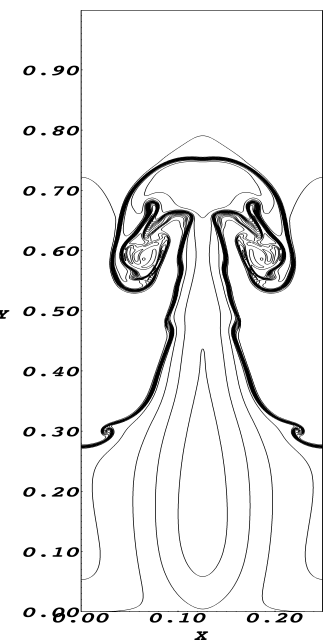

(e) Esquema 2

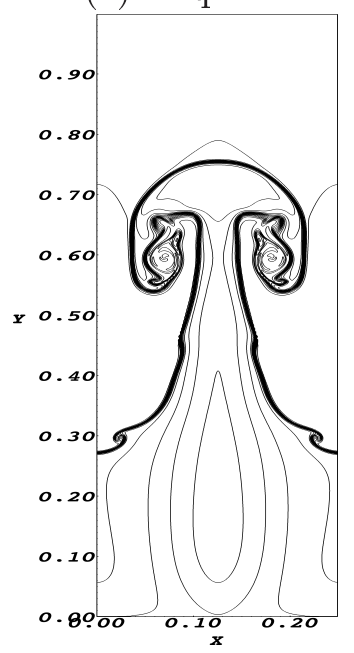

(h) Esquema 5

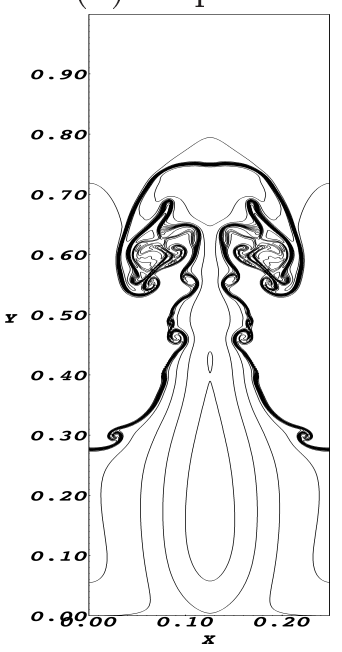

(c) EPUS

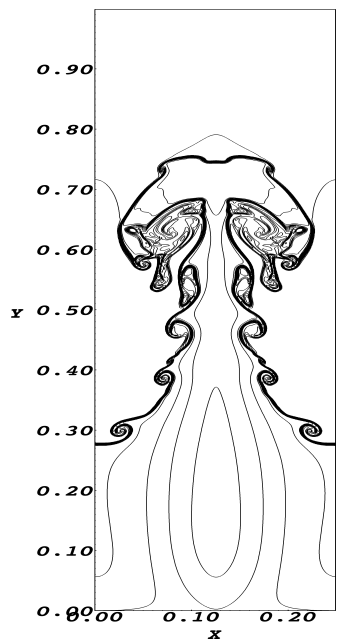

(f) Esquema 3

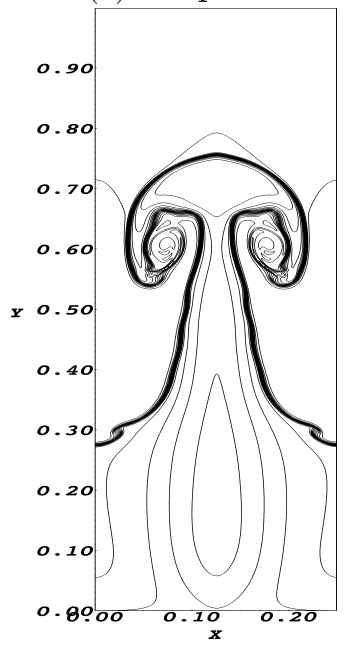

(i) Esquema 6

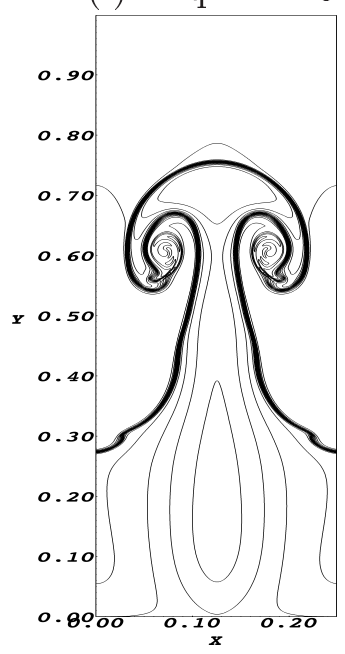

Figura 6.55: Perfil para a densidadde $\rho$ do fluido no Teste 13. (a) WENO5 (b) van Albada; (c) EPUS; (d)-(i) Esquemas 1-6 com os melhores parâmetros na malha $240 \times 960$ (grossa). 
(a) Weno5

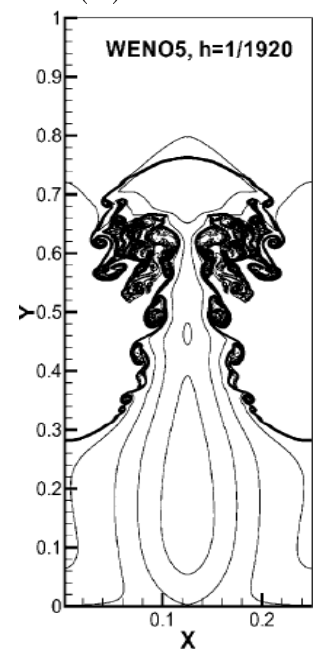

(d) Esquema 1

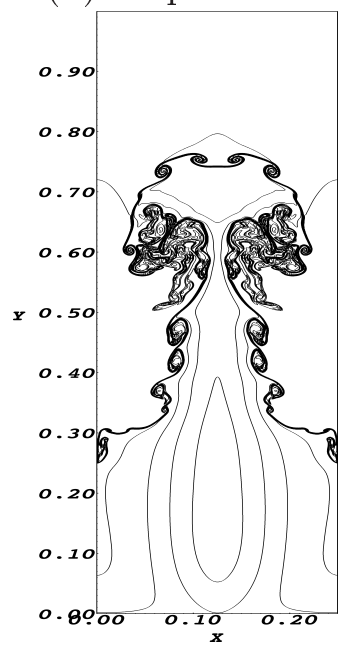

(g) Esquema 4

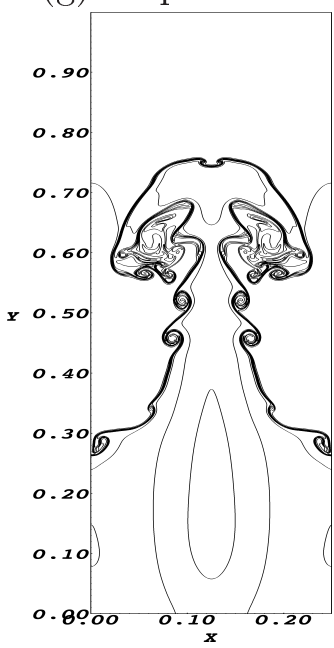

(b) van Albada

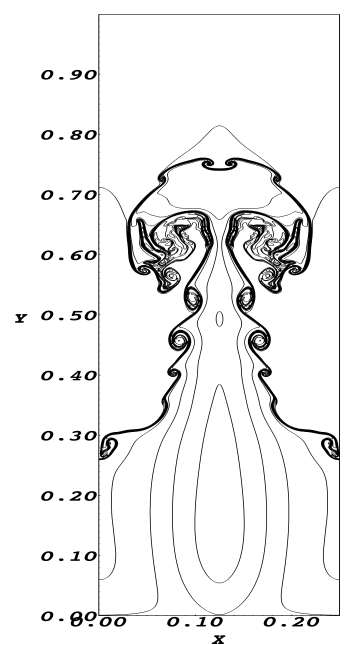

(e) Esquema 2

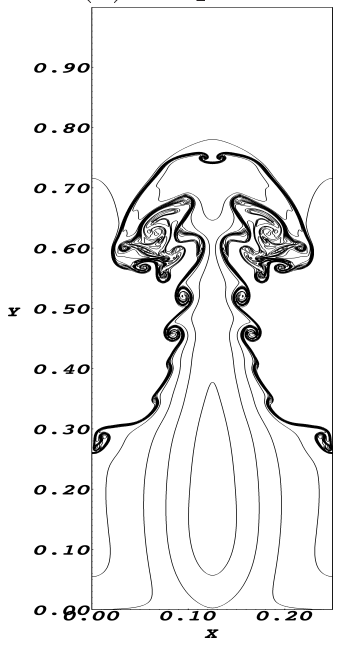

(h) Esquema 5

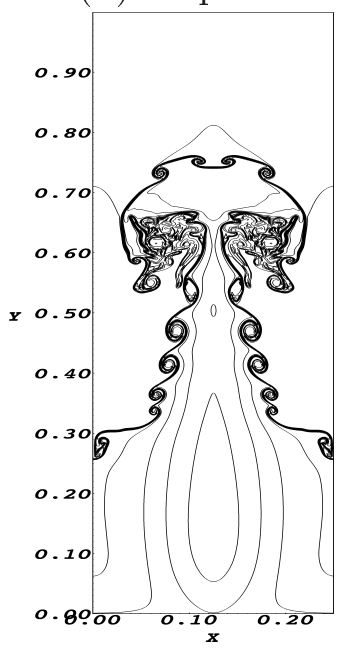

(c) EPUS

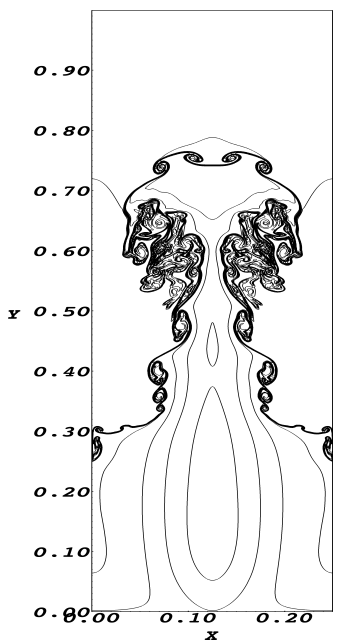

(f) Esquema 3

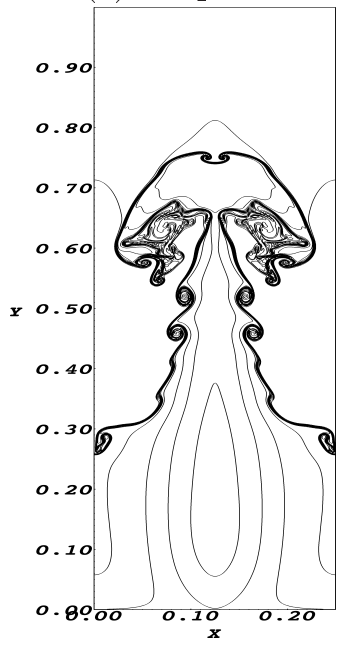

(i) Esquema 6

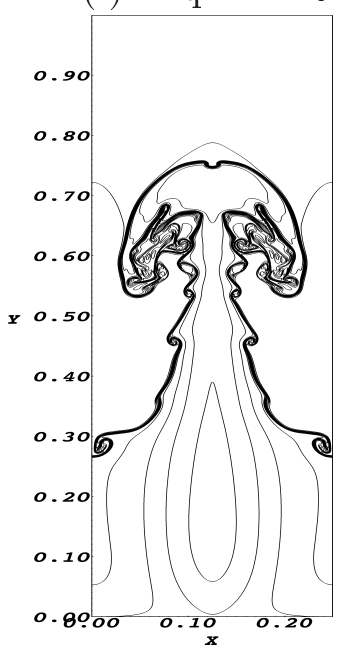

Figura 6.56: Perfil para a densidade $\rho$ do fluido no Teste 13. (a) WENO5 (b) van Albada; (c) EPUS; (d)-(i) Esquemas 1-6 com os melhores parâmetros na malha $480 \times 1920$ (fina). 


\section{Aplicação do Esquema 1 às equações de Navier-Stokes}

Neste capítulo são apresentados resultados computacionais usando-se o Esquema 1 (com $\beta=0.1545$ ) para problemas complexos de escoamentos de fluidos newtonianos envolvendo superfícies livres móveis. Esses problemas são modelados pelas equações de Navier-Stokes incompressíveis e simulados para os casos 2D, 2.5D (simetria radial) e 3D. O propósito das simulações apresentadas neste capítulo é mostrar que o Esquema 1 é útil também na simulação desses problemas complexos com superfícies livres. O Esquema 1 com esse valor de $\beta$ foi selecionado em virtude dos bons resultados obtidos por ele em equações de conservação gerais simuladas no capítulo anterior.

\subsection{Simulação de problemas $2 \mathrm{D}$ e $2.5 \mathrm{D}$}

Os resultados apresentados a seguir são para escoamentos incompressíveis 2D e 2.5D modelados pelas equações (2.10)-(2.11) e (2.12)-(2.14), respectivamente.

\section{Jato livre sobre um parede rígida}

Nesta primeira aplicação o Esquema 1 é usado para simular uma jato livre incidindo perpendicularmente numa superfície rígida impermeável, como ilustrado na Figura 7.1. Para a simulação desse problema, foram utilizados os seguintes dados::

$\diamond$ Domínio: $0.1 m \times 0.025 m$;

$\diamond$ Malha I: $100 \times 25 \Rightarrow \delta x=\delta y=0.001$; 


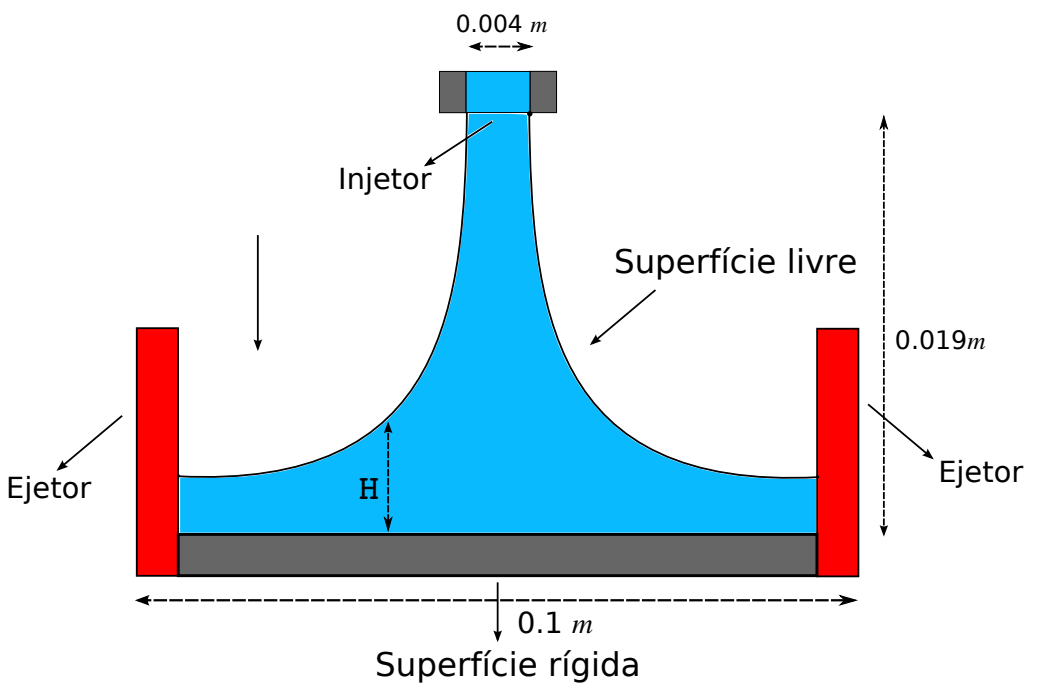

Figura 7.1: Esquematização de um jato livre incidindo perpendicularmente sobre uma superfície rígida impermeável

$\diamond$ Malha II: $200 \times 50 \Rightarrow \delta x=\delta y=0.0005$

$\diamond$ Malha III: $400 \times 100 \Rightarrow \delta x=\delta y=0.00025$;

$\diamond$ Malha IV: $800 \times 200 \Rightarrow \delta x=\delta y=0.000125$;

$\diamond$ Raio do injetor: $a=0.002 m$;

$\diamond$ Distância ao injetor: $h_{i}=0.019 m$;

$\diamond$ Numero de Reynolds: $R_{e}=2000$;

$\diamond$ Gravidade: $g=9.81 \mathrm{~m} / \mathrm{s}^{2}$;

$\diamond$ Escala de velocidade: $U_{0}=1 \mathrm{~m} / \mathrm{s}$;

$\diamond$ Escala de comprimento: $L_{0}=2 a=0.004$.

A Figura 7.2 apresenta a evolução do escoamento, mostrando os campos de velocidade em $x, y$ e o campo de pressão $p$ obtidos com a malha $I V$.

Este problema tem solução analítica (ver [51]) para a altura $H$ da superfície livre dada por

$$
H(x)= \begin{cases}\frac{\pi}{\sqrt{3}} \frac{\nu(x+l)}{Q}, & x \geq x_{0}, \\ a+\left(\frac{1-2 \pi}{3 \sqrt{3} c^{2}}\right) \delta(x), & x<x_{0}\end{cases}
$$

onde

$$
\begin{gathered}
Q=a U_{0}, \quad c=1.402, \quad x_{0}=\left(\frac{3 \sqrt{3} c(\pi-c) \sqrt{3}}{2 \pi^{2}}\right) a R_{e}, \\
l=\left(\frac{3 \sqrt{3} c(2 \sqrt{3} c-\pi) \sqrt{3}}{2 \pi^{2}}\right) a R_{e}, \quad \delta^{2}(x)=\frac{3 \sqrt{3} c^{3}}{2(\pi-c \sqrt{3})} \frac{\nu x}{U_{0}} .
\end{gathered}
$$


Contorno da velocidade na direção $\mathrm{x}$
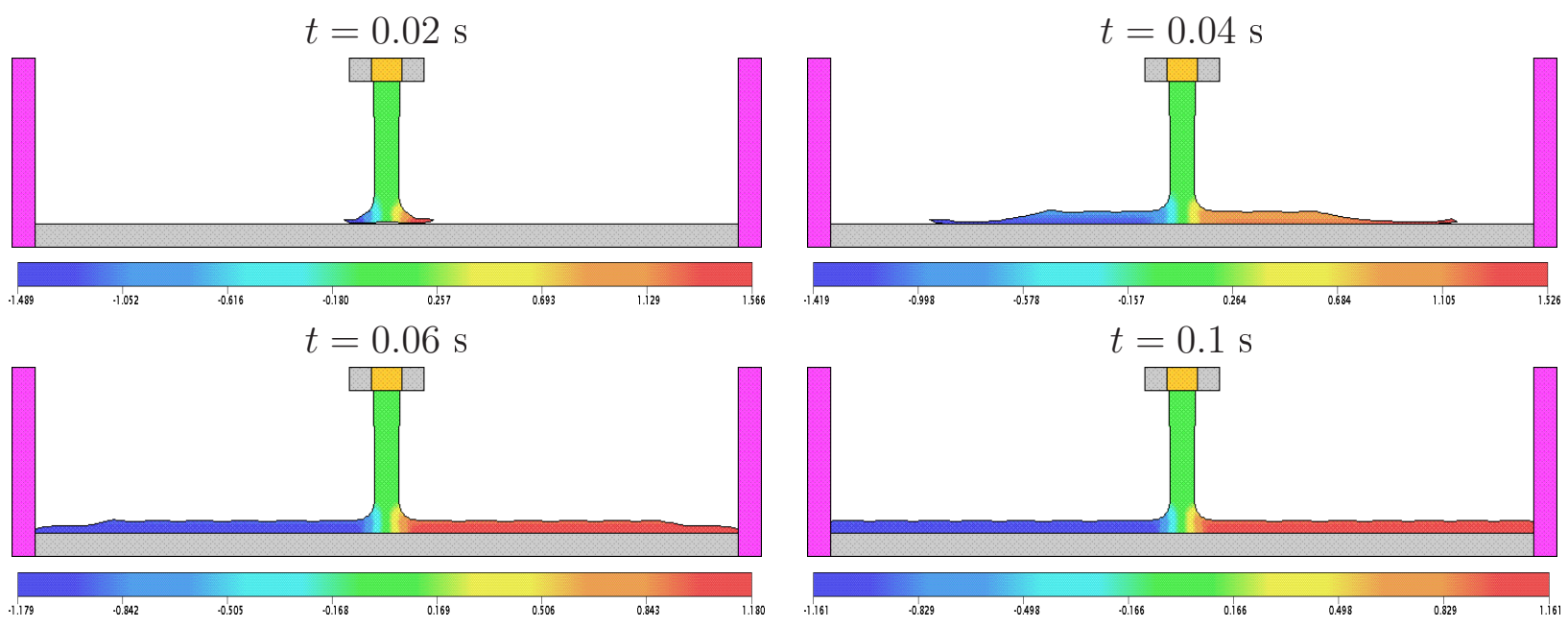

Contorno da velocidade na direção y
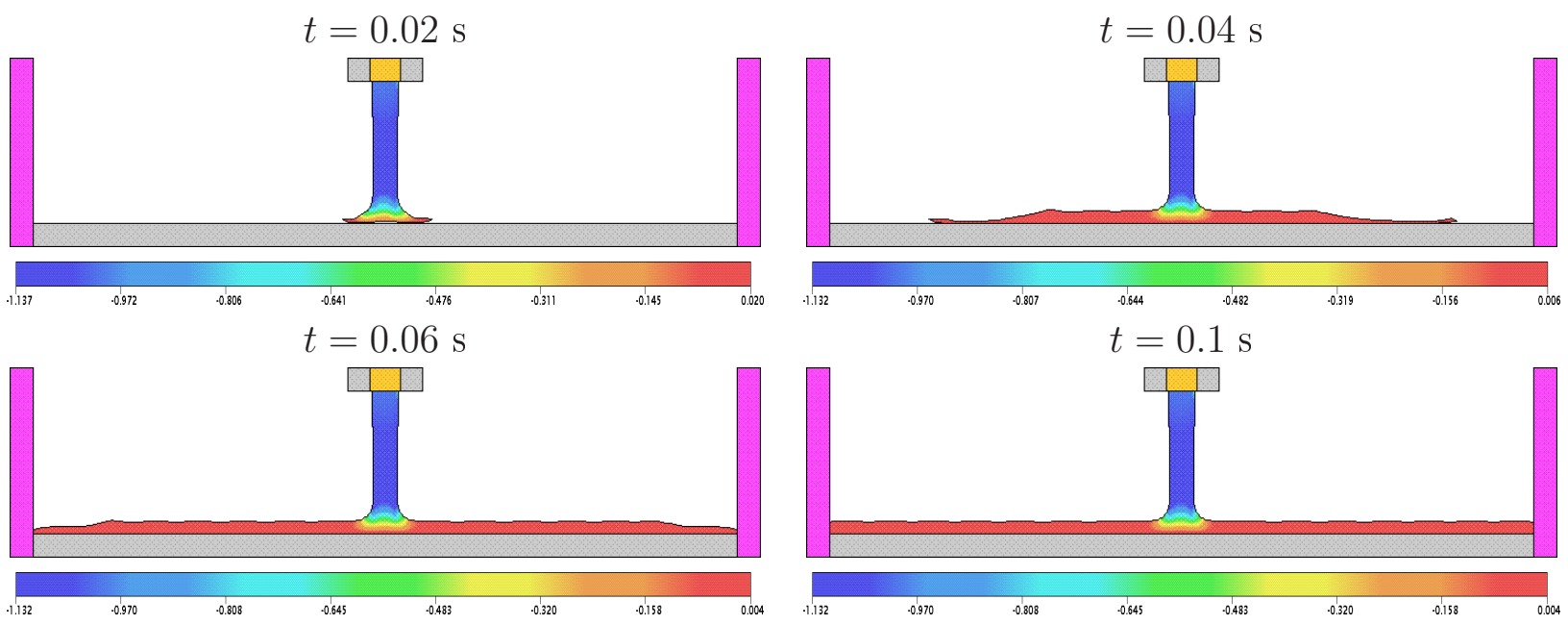

\section{Contorno da pressão}
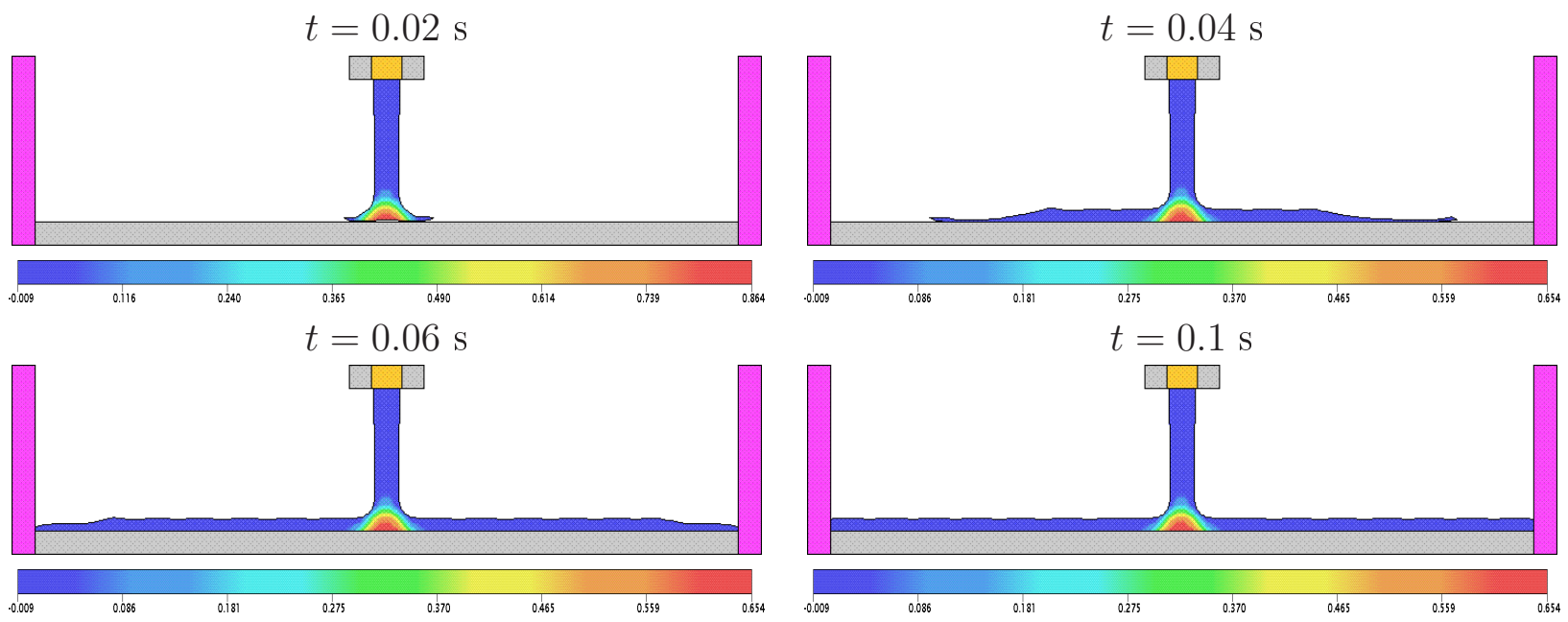

Figura 7.2: Contornos da velocidade em x, em y e da pressão para o problema do jato livre em diferentes tempos. 
A Figura 7.3 mostra a comparação da solução numérica nas malhas I, II, III e IV com a solução analítica de Watson [51]. Por essa figura, é possível notar que as soluções numéricas têm uma tendência em convergir para a solução analítica para valores pequenos do adimensional $x /\left(0.5 L_{0} R_{e}\right)$.

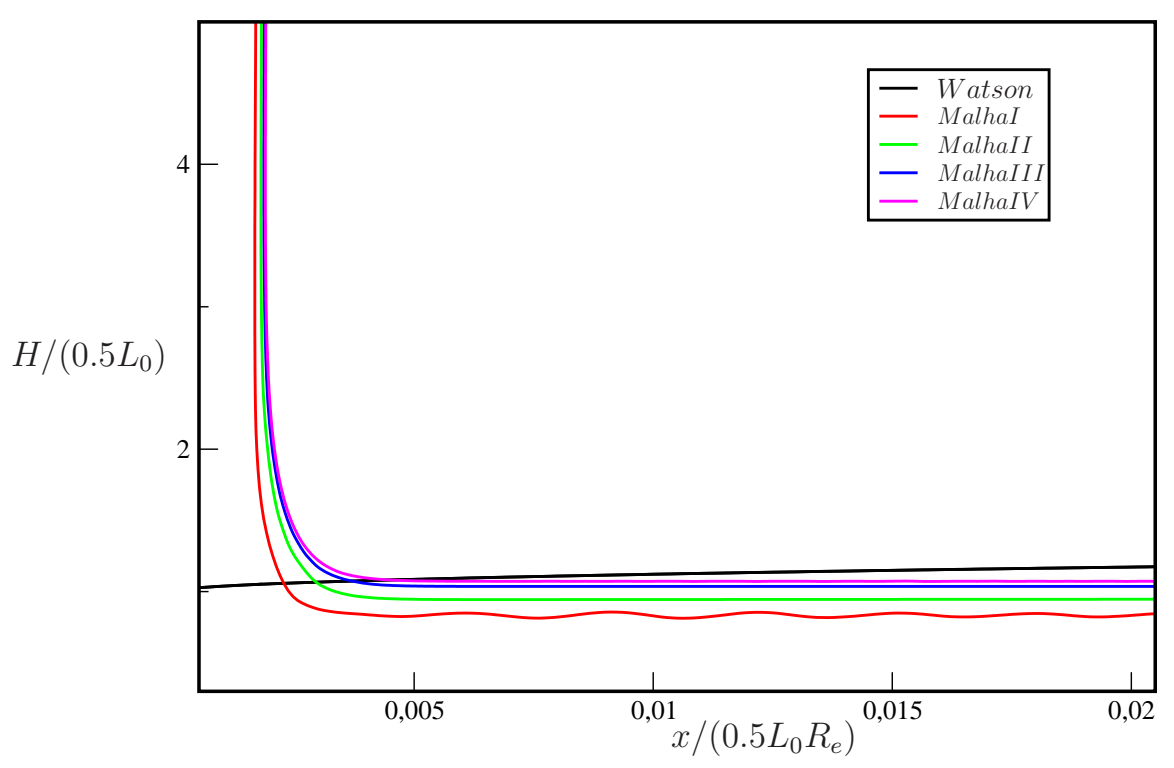

Figura 7.3: Comparação entre a solução analítica de Watson [51] e a solução numérica para diferentes malhas.

\section{Colapso de coluna de fluido}

Nesta aplicação considera-se um bloco de fluido de lados $b$ em equilíbrio hidrostático e sob a ação do campo gravitacional. O bloco é posto em movimento e o fluido escoa sobre uma superfície rígida impermeável, conforme ilustra a Figura 7.4.

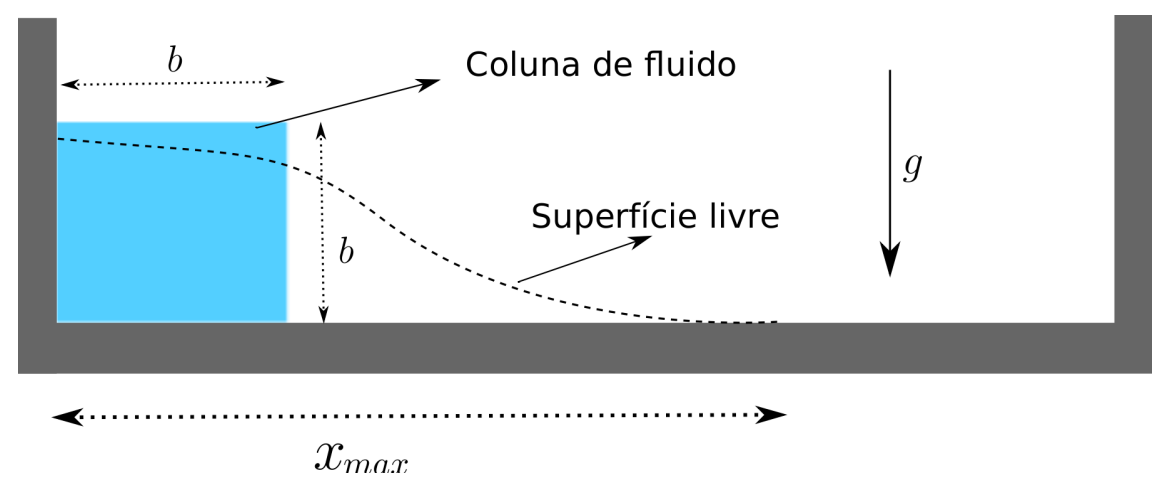

Figura 7.4: Esquematização do problema de colapso de uma coluna de fluido 2D.

Os seguintes dados foram utilizados na simulação:

$\diamond$ Domínio: $0.5 m \times 0.1 m$; 
$\diamond$ Malha: $1000 \times 200 \Rightarrow \delta x=\delta y=0.0005$;

$\diamond$ Dimensão da coluna: $b=0.057 m$;

$\diamond$ Numero de Reynolds: $R_{e}=42623.27$;

$\diamond$ Escala de velocidade: $U_{0}=\sqrt{g L_{0}}=0.74778 \mathrm{~m} / \mathrm{s}$;

$\diamond$ Escala de comprimento: $L_{0}=b=0.057$.

O valor máximo do espalhamento horizontal $\left(x_{\max }\right)$ (ver Figura 7.4) é conhecido por dados experimentais devido a Martin e Moyce [27], e também via simulação numérica por Colagrossi e Landrini [6] usando técnicas numéricas consagradas. Estes dados foram confrontados com o espalhamento horizontal máximo obtido da solução numérica com o Esquema 1, como mostra a Figura 7.5. Por essa figura, constata-se que o código Freeflow equipado com o Esquema 1 simulou bem o problema, derivando uma solução numérica consistente com as soluções numéricas obtidas com as técnicas Level Set, BEM e SPH.

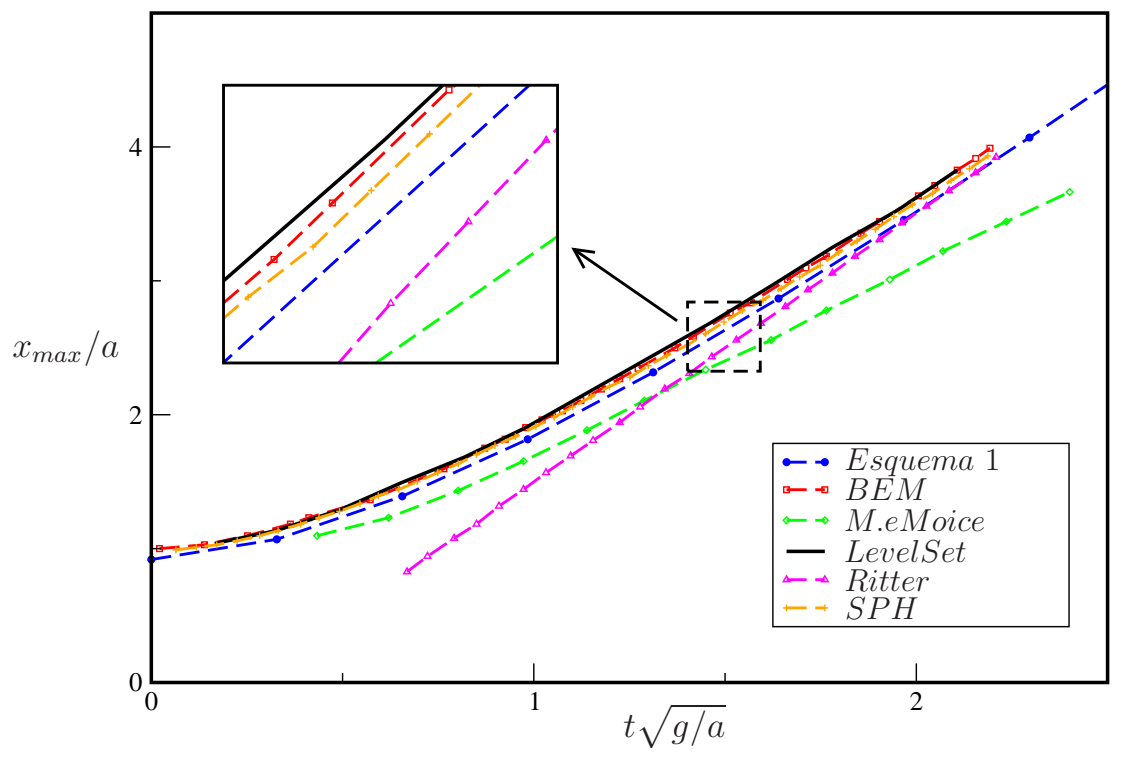

Figura 7.5: Comparação entre as soluções experimentais, numéricas e teóricas para o fenômeno do colapso de uma coluna de fluido.

Para ilustração, a Figura 7.6 mostra a evolução do escoamento, mostrando os campos de velocidade e pressão.

\section{Experimento de Taylor}

Simula-se nesta seção o experimento de Taylor [42], que consiste em um jato de fluido incidindo perpendicularmente numa porção do mesmo fluido em repouso. Uma esquematização do problema em questão é apresentada na Figura 7.7. Para simulação numérica do problema, na qual a superfície rígida é um contorno sem escorregamento, utilizam-se os seguintes dados: 


\section{Contorno da velocidade na direção $\mathrm{x}$}

$$
t=0.025 \mathrm{~s}
$$

$t=0.075 \mathrm{~s}$
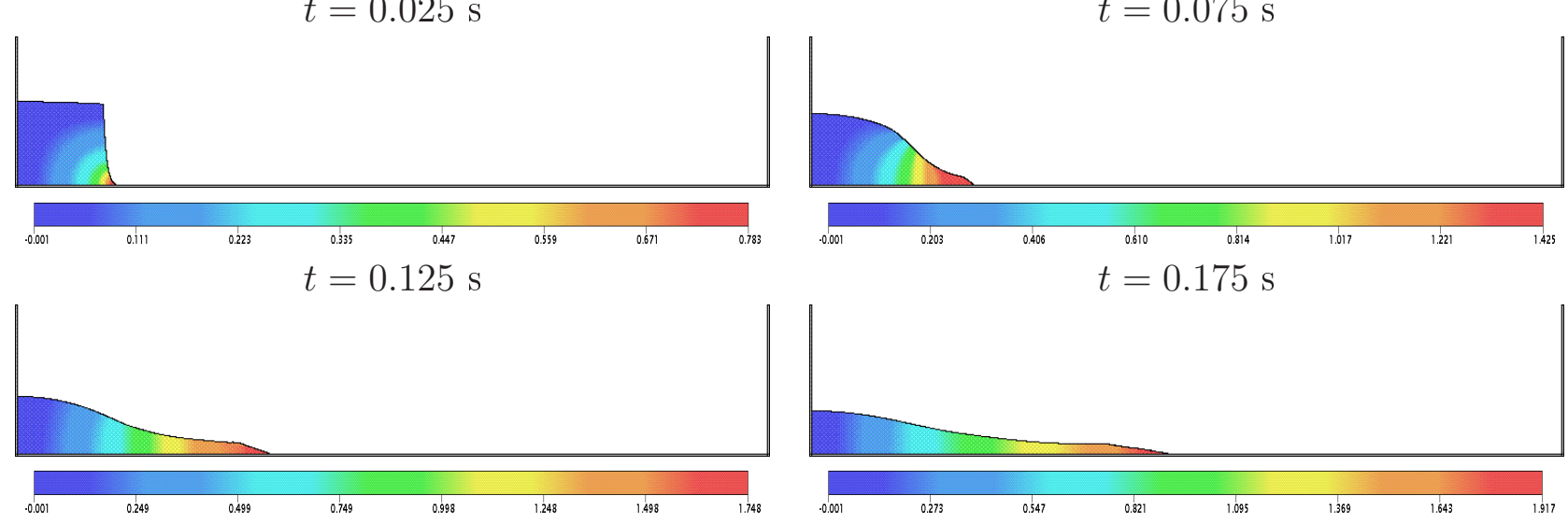

Contorno da velocidade na direção y

$t=0.025 \mathrm{~s}$
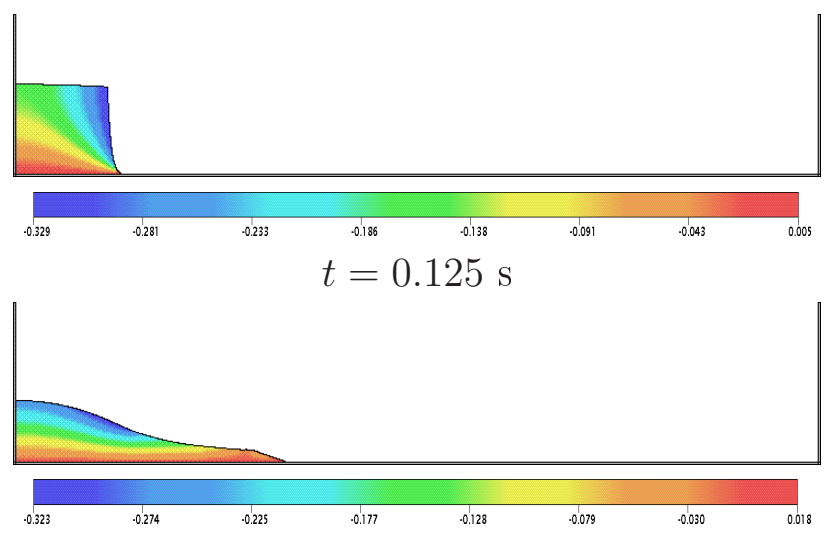

$t=0.075 \mathrm{~s}$
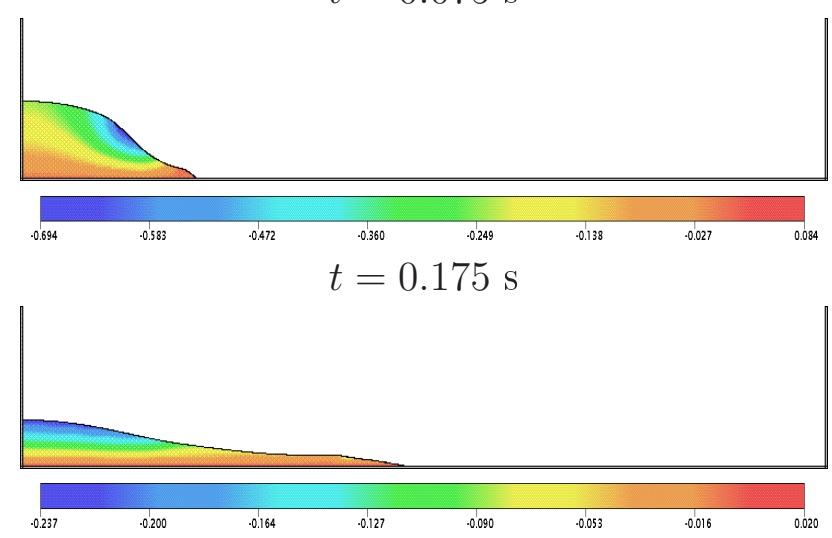

Contorno da pressão

$t=0.025 \mathrm{~s}$
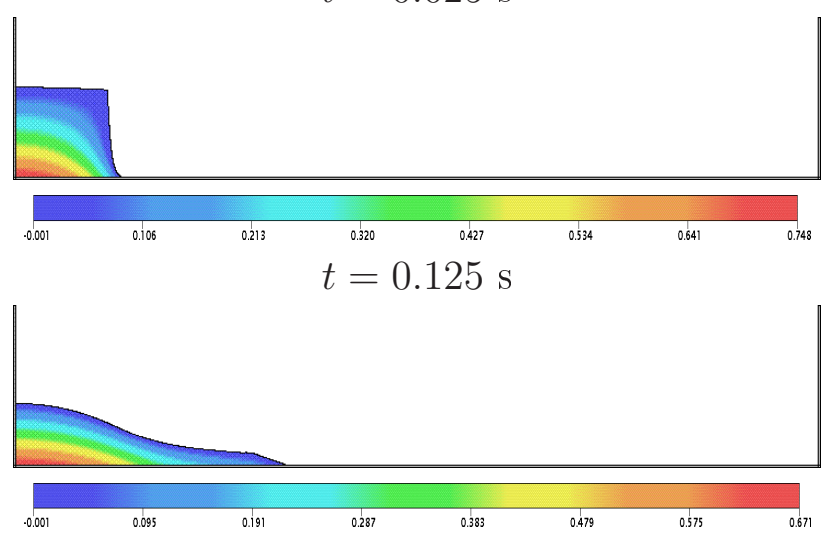

$$
t=0.075 \mathrm{~s}
$$
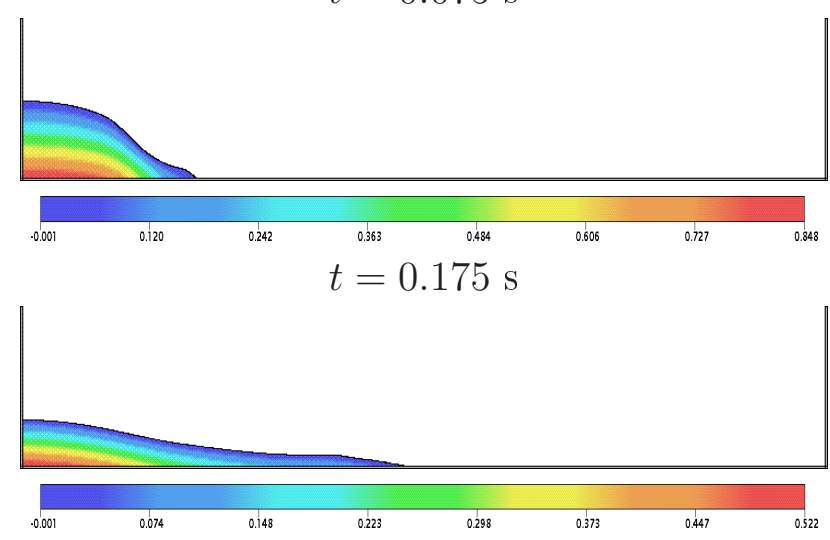

Figura 7.6: Contornos da velocidade em x, em y e da pressão para o problema do colapso de uma coluna de fluido em $2 \mathrm{D}$ para diferentes tempos. 


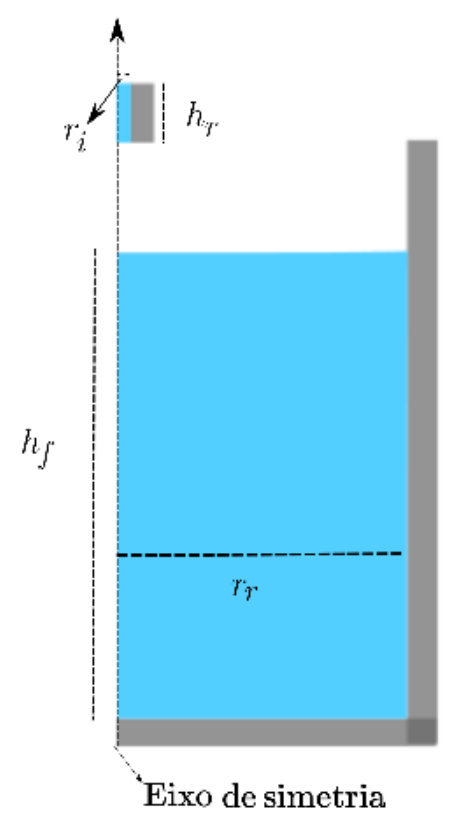

Figura 7.7: Esquematização para o experimento de Taylor.

$\diamond$ Domínio: $0.0615 m \times 0.2015 m$;

$\diamond$ Malha: $123 \times 403 \Rightarrow \delta x=\delta y=0.0005$;

$\diamond$ Raio do cilindro: $r_{r}=0.06 m$;

$\diamond$ Raio do injetor: $r_{i}=0.002 m$;

$\diamond$ Altura do injetor: $h_{i}=0.03 m$;

$\diamond$ Altura do fluido: $h_{f}=0.16 m$;

$\diamond$ Numero de Reynolds: $R_{e}=200$;

$\diamond$ Escala de velocidade: $U_{0}=0.5 \mathrm{~m} / \mathrm{s}$;

$\diamond$ Escala de comprimento: $L_{0}=2 r_{i}=0.004 m$.

A Figura 7.8 apresenta a solução experimental de Taylor e a simulação computacional com o Esquema 1 nos tempos $t=0.75$ e $t=2.5$. Observa-se nas comparações que os resultados numéricos com o Esquema 1 capturaram com sucesso as estruturas em forma de toro, mostrando bastante consistência com o resultado experimental. Para simples ilustração, a Figura 7.9 apresenta a evolução desse escoamento complexo com superfícies livres em movimento. Apresenta-se também na Figura 7.10 o escoamento no tempo $t=7 \mathrm{~s}$, mostrando a formação de uma estrutura complexa durante o movimento do fluido incidente. 
Perfil da solução no tempo $t=0.75 \mathrm{~s}$

(a)

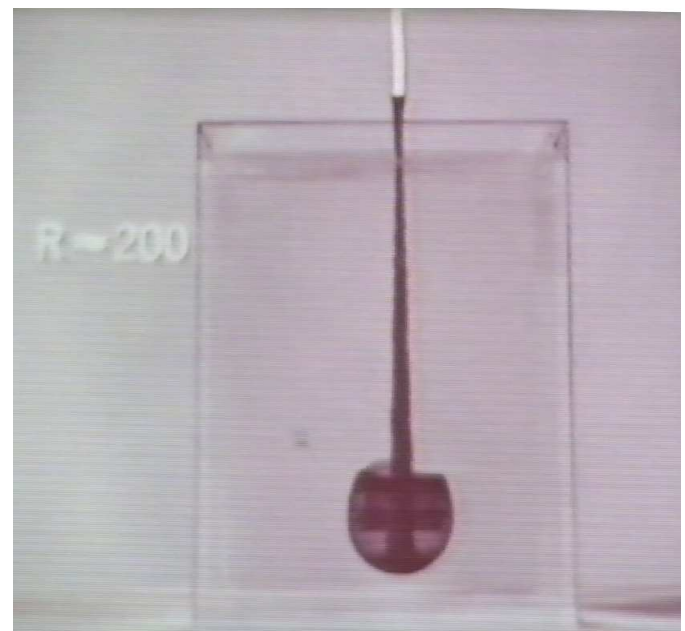

Perfil da solução no tempo $t=2.5 \mathrm{~s}$

(c)

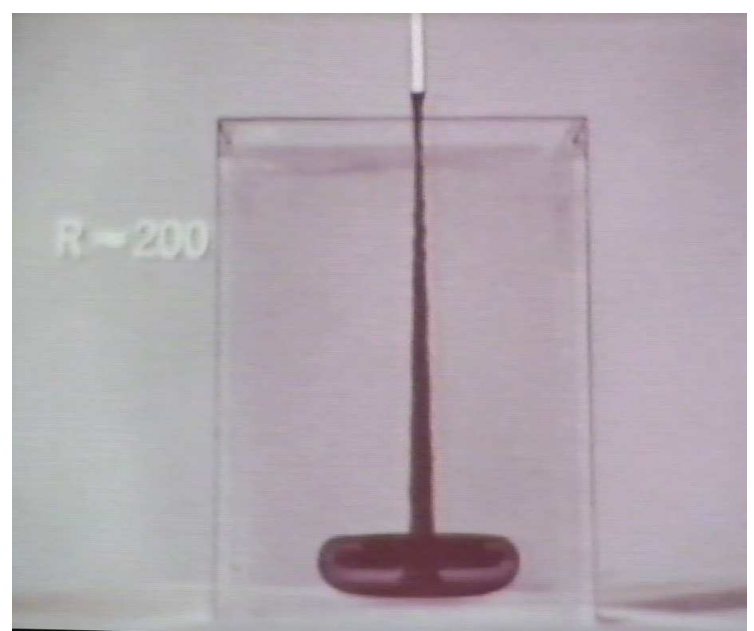

(b)

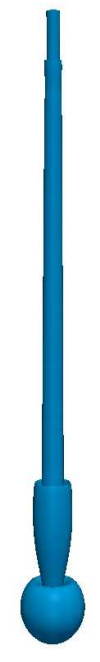

(d)

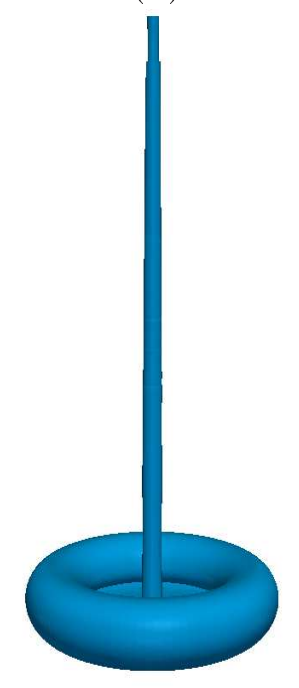

Figura 7.8: Ilustração do experimento de Taylor em diferentes tempos. (a) e (c) solução experimental; (b) e (d) solução numérica. 


\section{Evolução temporal para o experimento de Taylor}

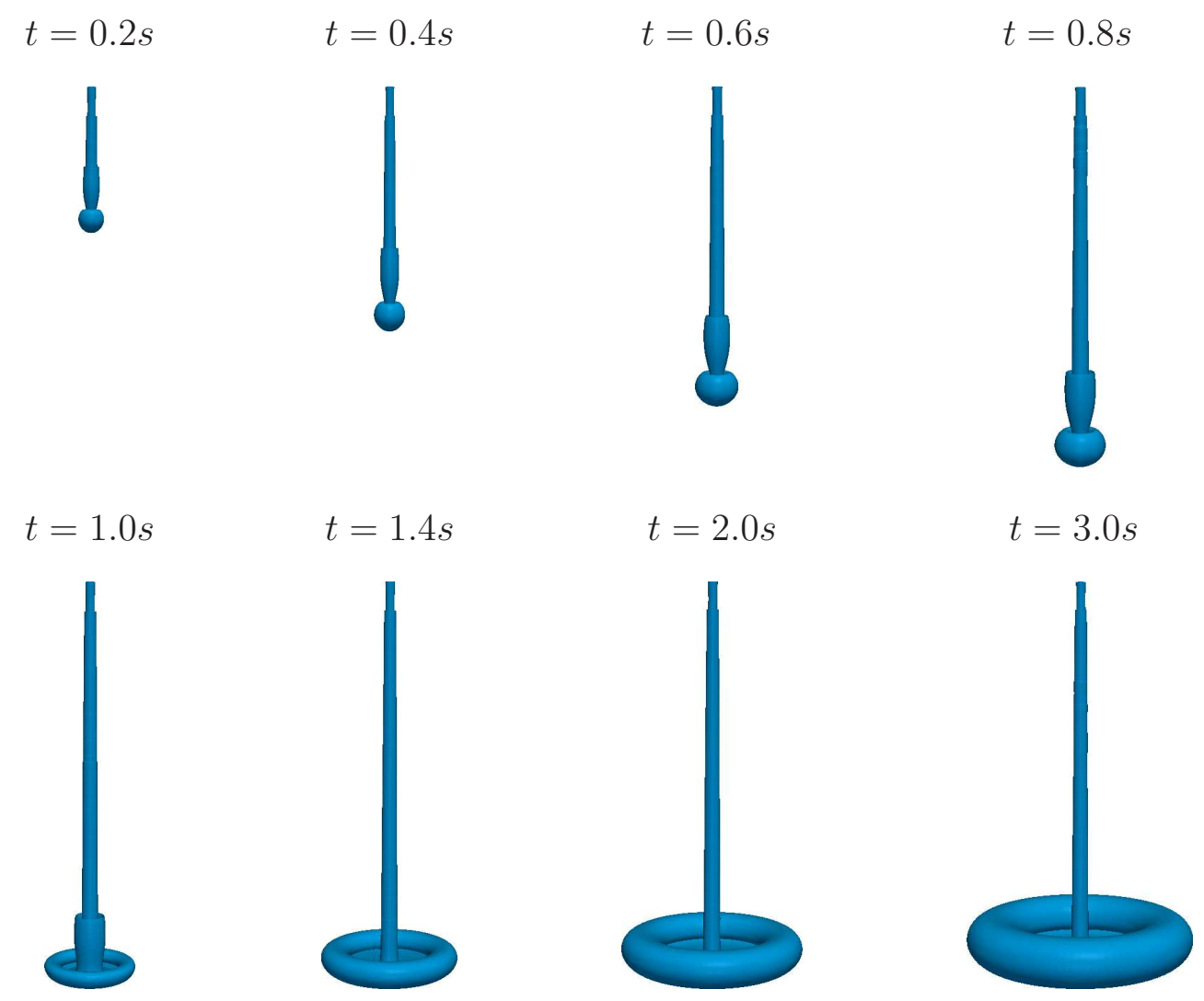

Figura 7.9: Ilustração da solução para o experimento de Taylor em diferentes tempos.

$$
t=7 s
$$
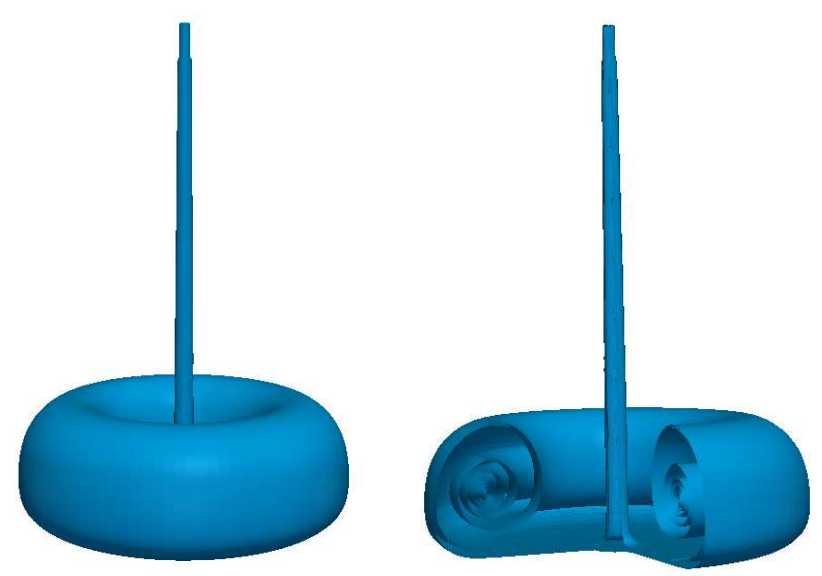

Figura 7.10: Ilustração do experimento de Taylor em $T=7 \mathrm{~s}$ e corte transversal evidenciando a formação de estruturas complexas. 


\section{Ressalto hidráulico circular}

O fenômeno interessante denominado ressalto hidráulico circular é simulado nesta seção. Tal fenômeno, útil para testar esquemas upwind, aparece quando um jato livre incide perpendicularmente sobre uma superfície rígida sob a ação da gravidade. Sob certas condições do escoamento, sobre os números de Reynolds e de Froude, o ressalto hidráulico circular é acompanhado por perda de energia e produção de turbulência. O conhecimento da altura da película de fluido antes e depois do ressalto permite a determinação de taxas de transferência de calor na superfície (ver [34]). A Figura 7.11 ilustra o fenômeno.

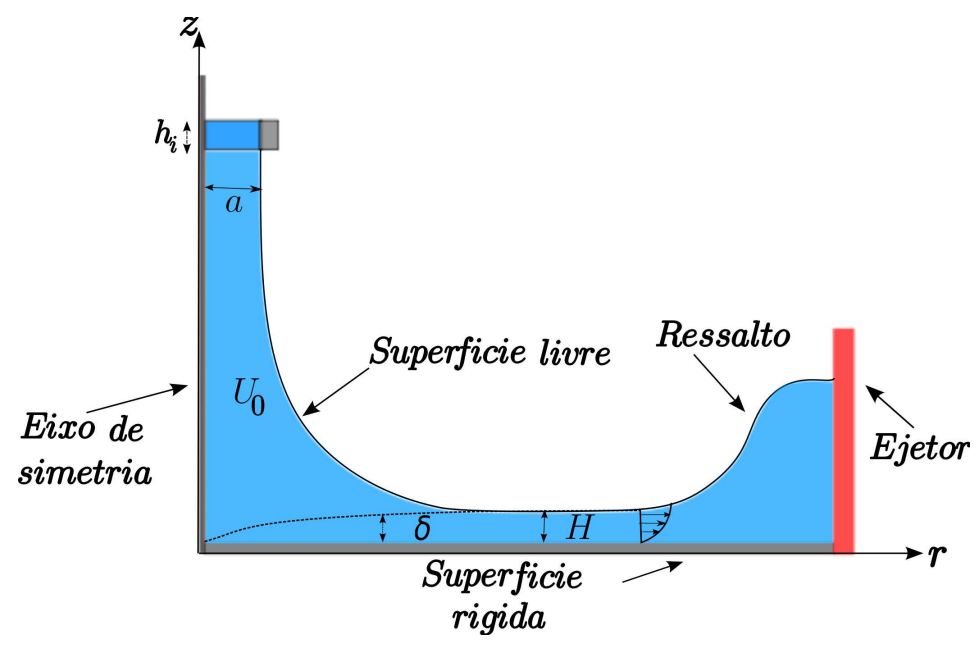

Figura 7.11: Esquematização para o fenômeno do ressalto hidráulico circular.

Para a simulação do fenômeno ressalto hidráulico, foi aplicada a condição de não escorregamento no contorno rígido e foram usados os seguintes dados:

$\diamond$ Domínio: $0.05 m \times 0.0315 m$;

$\diamond$ Malha I: $200 \times 126 \Rightarrow \delta x=\delta y=0.00025$;

$\diamond$ Malha II: $400 \times 252 \Rightarrow \delta x=\delta y=0.000125$;

$\diamond$ Malha III: $800 \times 504 \Rightarrow \delta x=\delta y=0.0000625$;

$\diamond$ Raio do injetor: $a=0.004 m$;

$\diamond$ Altura do injetor: $h_{i}=0.00075 m$;

$\diamond$ Numero de Reynolds: $R_{e}=250$;

$\diamond$ Escala de velocidade: $U_{0}=0.375 \mathrm{~m} / \mathrm{s}$;

$\diamond$ Escala de comprimento: $L_{0}=2 a=0.008 m$.

Para verificar a solução obtida via esquema numérico, é feita a comparação com a solução analítica de Watson [51] dada por 


$$
H(r)= \begin{cases}\frac{a^{2}}{2 r}+\left(1-\frac{2 \pi}{3 \sqrt{3} c^{2}}\right) \delta, & r<r_{0}, \\ \frac{2 \pi^{2}}{3 \sqrt{3}} \frac{\nu\left(r^{3}+l^{2}\right)}{Q r}, & r \geq r_{0},\end{cases}
$$

em que

$$
\delta=\sqrt{\frac{\pi \sqrt{3} c^{3}}{\pi-c \sqrt{3}} \frac{\nu r a^{2}}{Q}},
$$

sendo $r_{0}=0.3155 a R_{e}^{\frac{1}{3}}$ e $l=0.567 a R_{e}^{\frac{1}{3}}$. Vale ressaltar que esta solução analítica é valida após a região de impacto e antes do ressalto. Na região de interesse, é possível ver a partir da Figura 7.12 que, ao se refinar a malha, a solução numérica converge para uma solução próxima da solução de Watson. A Figura 7.13 mostra a comparação 3D da solução numérica com o experimento de Rai [34].

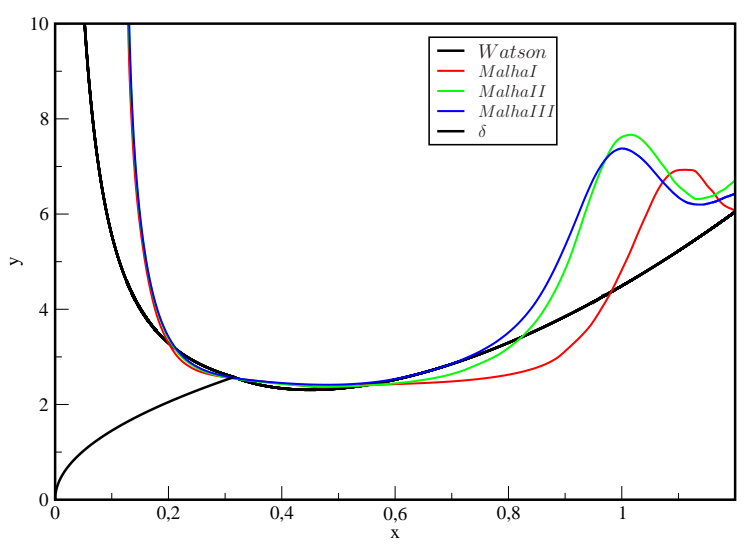

Figura 7.12: Comparação entre a solução analítica e a solução numérica para o ressalto hidráulico circular, considerando diferentes malhas.

(a)

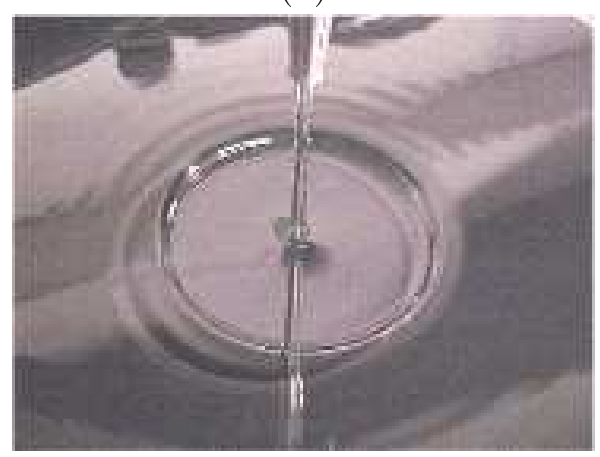

(b)

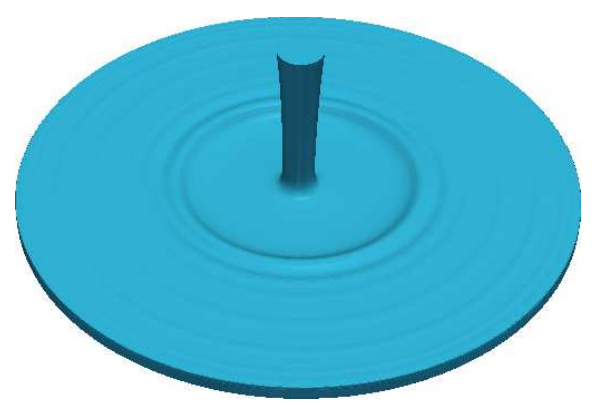

Figura 7.13: Ilustração do ressalto hidráulico circular. (a) solução experimental de Rai [34] (b) solução numérica. 


\subsection{Simulação de problemas 3D}

Os resultados apresentados a seguir são para escoamentos incompressíveis 3D modelados pelas equações (2.10)-(2.11).

\section{Colapso de bloco de fluido 3D}

Nesta primeira simulação (similar à apresentada para o caso 2D), apresentam-se os resultados para o problema Broken-dam, esquematizado na Figura 7.14. Objetiva-se validar o Freeflow3D equipado com o Esquema 1 no caso tridimensional. Na simulação foram utilizados os seguintes dados:

$\diamond$ Domínio: $0.3 m \times 0.1 m \times 0.16 m$;

$\diamond$ Malha: $150 \times 50 \times 80 \Rightarrow \delta x=\delta y=\delta z=0.002$;

$\diamond$ Numero de Reynolds: $R_{e}=99045.444$;

$\diamond$ Escala de velocidade: $U_{0}=0.99045444 \mathrm{~m} / \mathrm{s}$;

$\diamond$ Escala de comprimento: $L_{0}=0.1 \mathrm{~m}$.

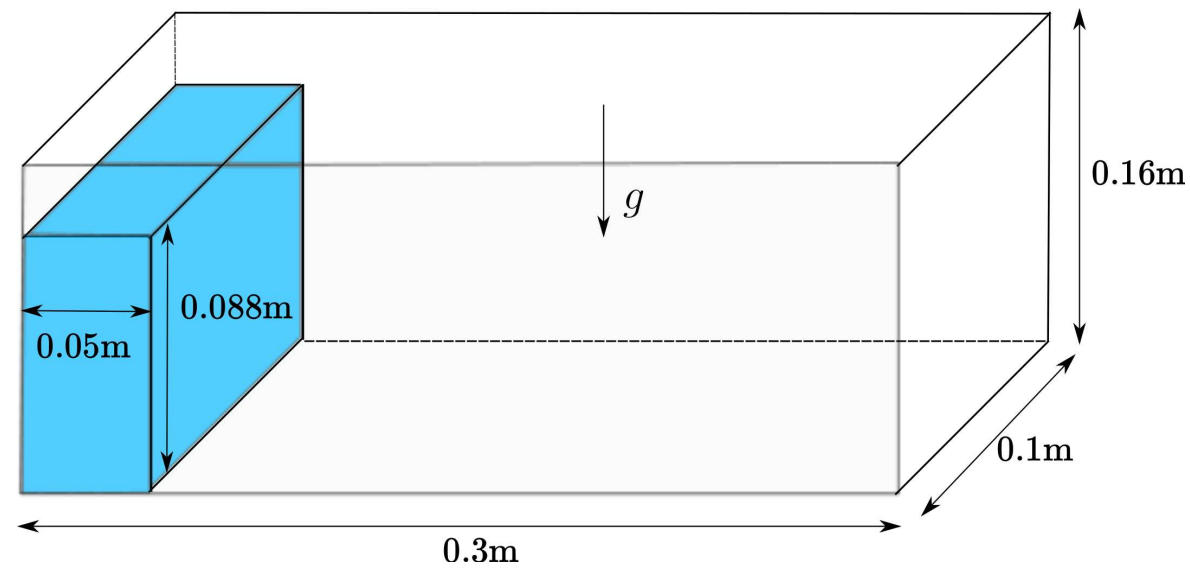

Figura 7.14: Esquematização para o colapso de fluido 3D.

A Figura 7.15 apresenta a solução numérica obtida com o Esquema 1 para o valor máximo do espalhamento horizontal $\left(x_{\max }\right)$ em comparação com os dados experimentais de Martin e Moyce [27], e numéricos de Colagrossi e Landrini [6]. É possível verificar que a solução numérica obtida com o Esquema 1 está em concordância com os dados da literatura.

A Figura 7.16 apresenta a evolução temporal da solução numérica, mostrando os campos de pressão e velocidade. 


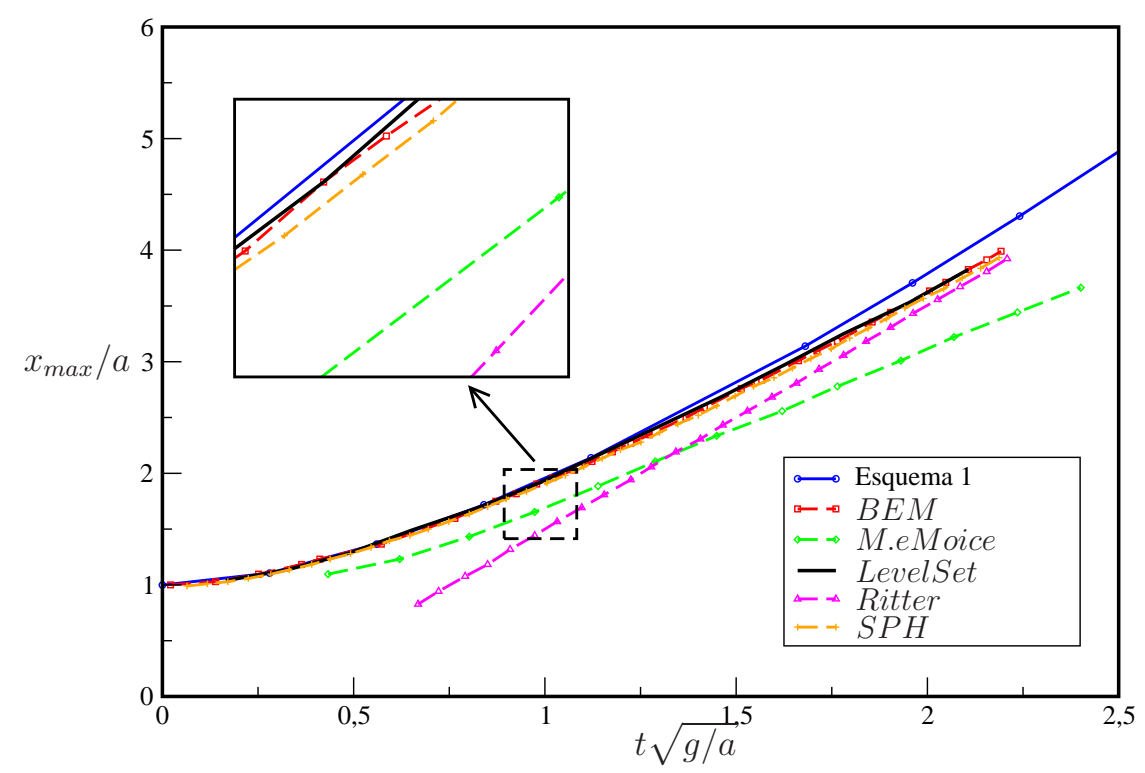

Figura 7.15: Comparação entre as soluções experimentais, numéricas e teóricas para o fenômeno do colapso de uma coluna de fluido em 3D.

\section{Jatos oscilantes}

Nesta aplicação, apresentam-se resultados computacionais para o problema clássico do jato oscilante: as instabilidades físicas em jatos livres altamente viscosos incidentes sobre superfícies rígidas. Este é um problema instável de grande interesse em mecânica dos fluidos, com várias aplicações (ver, por exemplo, [35] e [49]). Para o surgimento de instabilidades é necessário estabelecer condições favoráveis. Por exemplo, Cruickshank [8] propôs que para o aparecimento das instabilidades, a razão $H / d$ ( $H$ sendo a altura do injetor até a base e $d$ o diâmetro do injetor) deve ser maior que 7.2 e o número de Reynolds deve satisfazer $R_{e}<1.2$. Atualmente, o problema do jato oscilante é um excelente teste para validar métodos numéricos para escoamentos viscosos com superfícies livres. Objetiva-se com este teste mostrar que, muito embora tenha sido planejado para problemas a altos valores do número de Reynolds, o Esquema 1 é útil também em problemas em que $R_{e}<1$. A seguir apresentam-se três simulações do jato oscilante, com aplicação da condição de não escorregamento no contorno rígido.

\section{Jato planar}

Nesta simulação considera-se o caso do jato planar oscilante, em que o número de Reynolds $R_{e}$ e a razão $H / d$ são tomados como 0.72 e 50, respectivamente, condições estas satisfazendo a proposta de Cruickshank [8]. Na Figura 7.17 tem-se a representação esquemática do problema; e os dados restantes para a simulação são como segue:

$\diamond$ Domínio: $0.73 m \times 0.33 m \times 1.065 m$;

$\diamond$ Malha: $146 \times 66 \times 213 \Rightarrow \delta x=\delta y=\delta z=0.005$; 


\section{Campo de pressão}

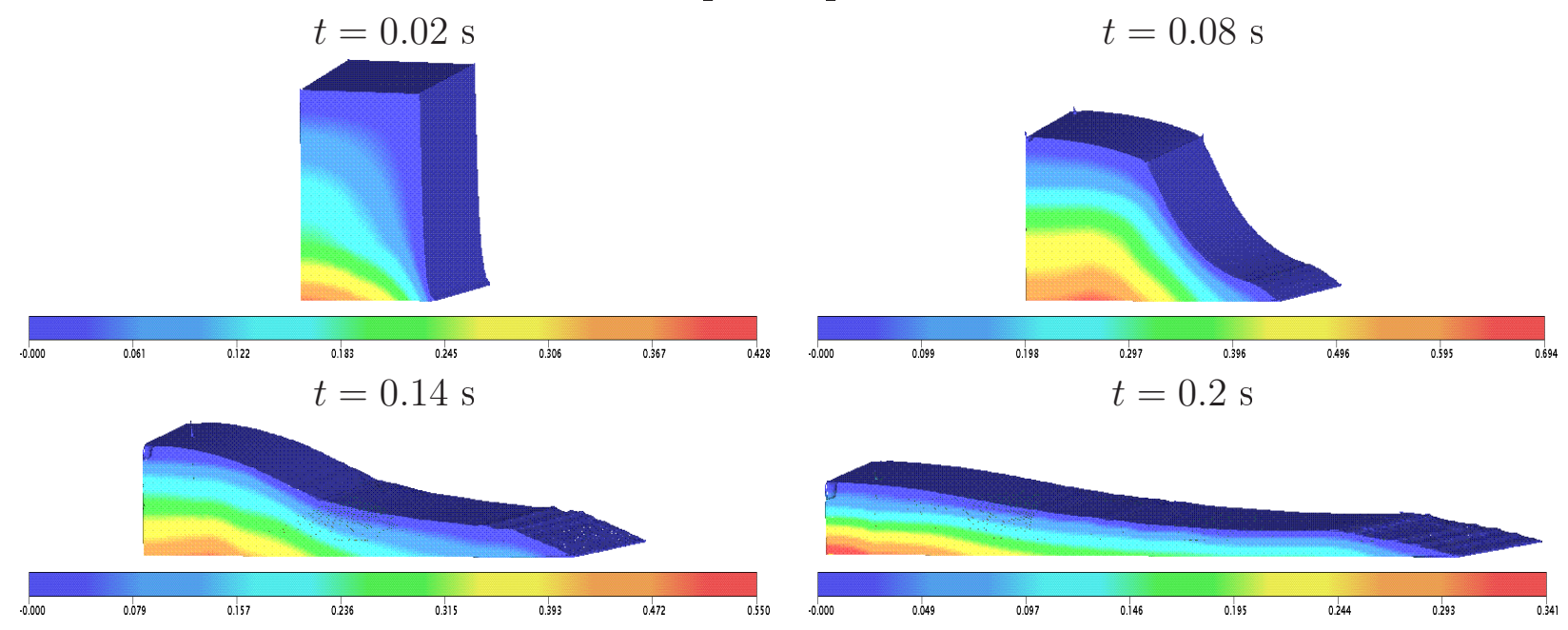

Campo de velocidade em $\mathrm{x}$
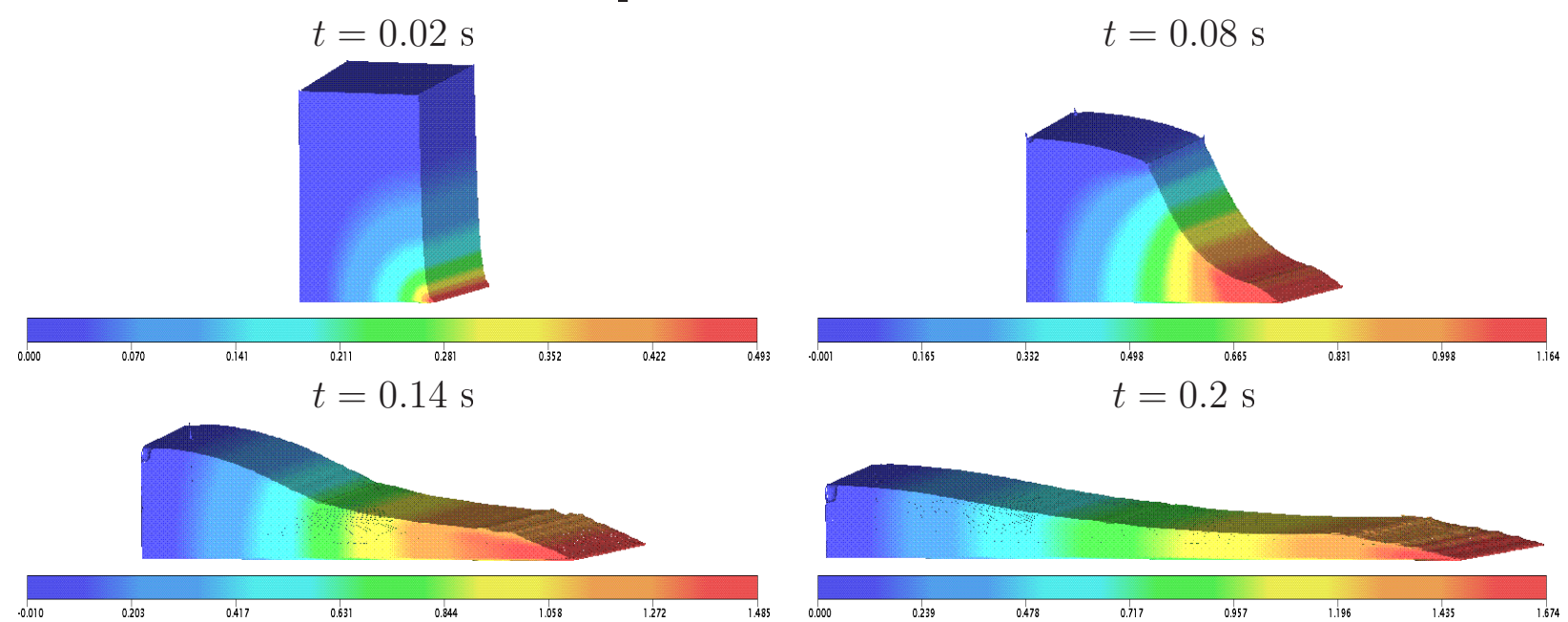

Campo de velocidade em $\mathrm{z}$
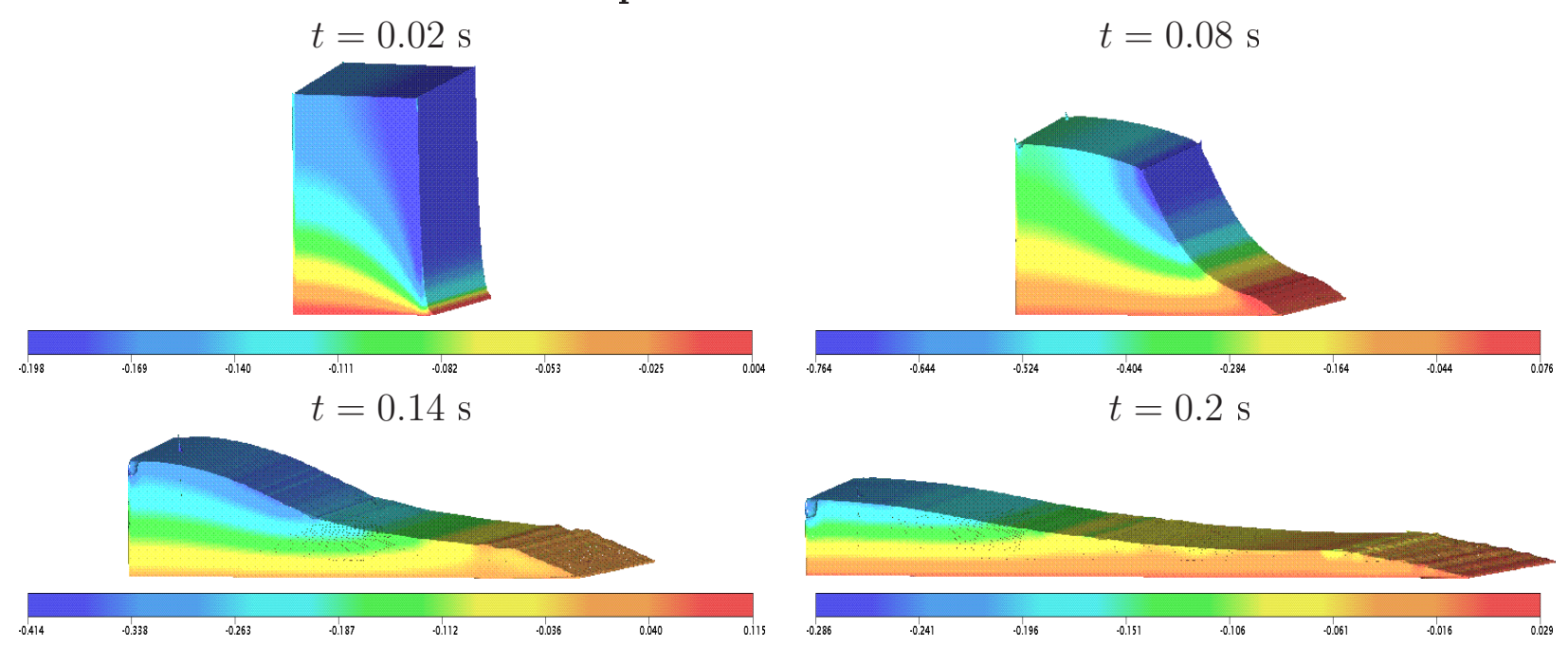

Figura 7.16: Campos de pressão e de velocidade em x e z para o problema do colapso de uma coluna de fluido em 3D para diferentes tempos. 
$\diamond$ Diâmetro do injetor: $d=0.02 m$;

$\diamond$ Comprimento do injetor: $L_{i}=0.2 m$;

$\diamond$ Altura do injetor: $h_{i}=0.05 m$;

$\diamond$ Altura livre: $H=1.0 \mathrm{~m}$;

$\diamond$ Escala de velocidade: $U_{0}=1 \mathrm{~m} / \mathrm{s}$;

$\diamond$ Escala de comprimento: $L_{0}=L_{i}=0.2 m$.

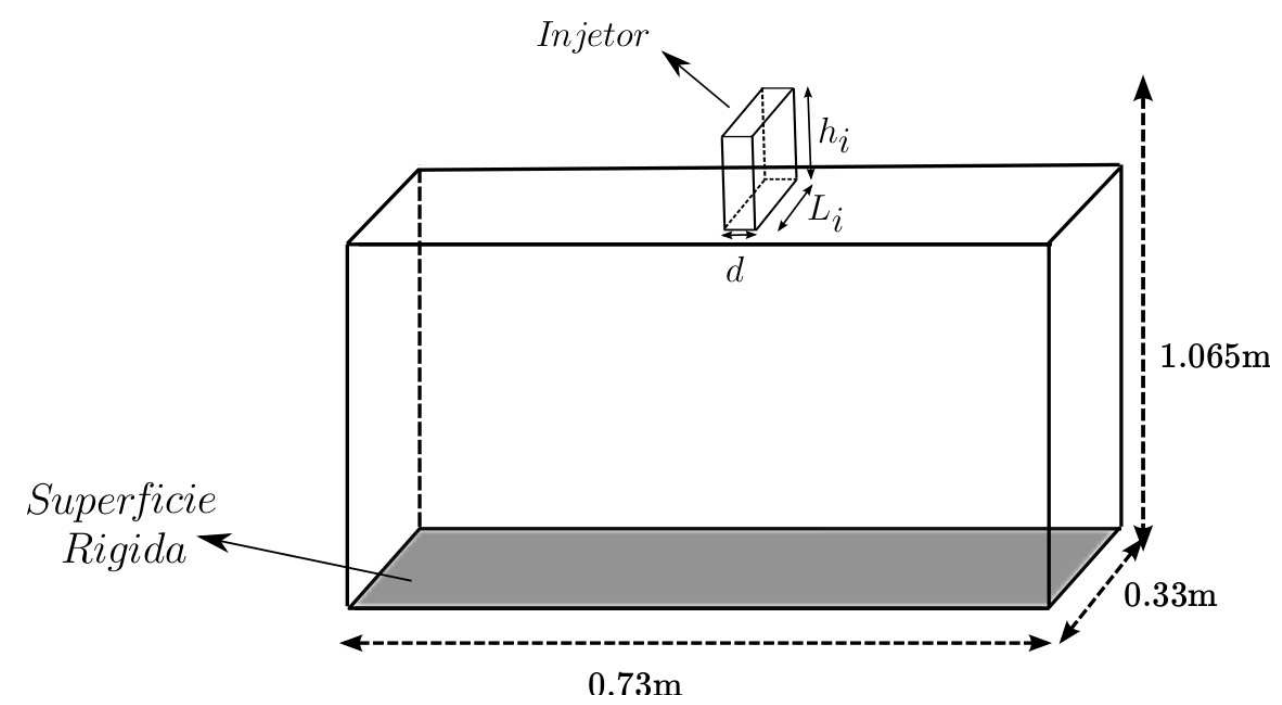

Figura 7.17: Esquematização para o problema do jato planar 3D.

Na Figura 7.18 estão os resultados da simulação em vários tempos, pode-se notar que o fenômeno de dobras (as instabilidades físicas) foi simulado com sucesso com o uso do Esquema 1.

\section{Jato circular}

Para o jato oscilante no caso do injetor circular, duas simulações foram feitas: uma delas (Caso 1) com diâmetro do injetor $d=0.08 \mathrm{~m}$ e a outra (Caso 2) com $d=0.04 \mathrm{~m}$. A Figura 7.19 ilustra esquematicamente o problema.

\section{Caso 1}

Para a simulação, utilizou-se os seguintes dados:

$\diamond$ Domínio: $1 m \times 1 m \times 1.26 m$;

$\diamond$ Malha: $100 \times 100 \times 126 \Rightarrow \delta x=\delta y=\delta z=0.01$;

$\diamond$ Numero de Reynolds: $R_{e}=0.288$; 


\section{Evolução temporal para o problema do jato planar.}
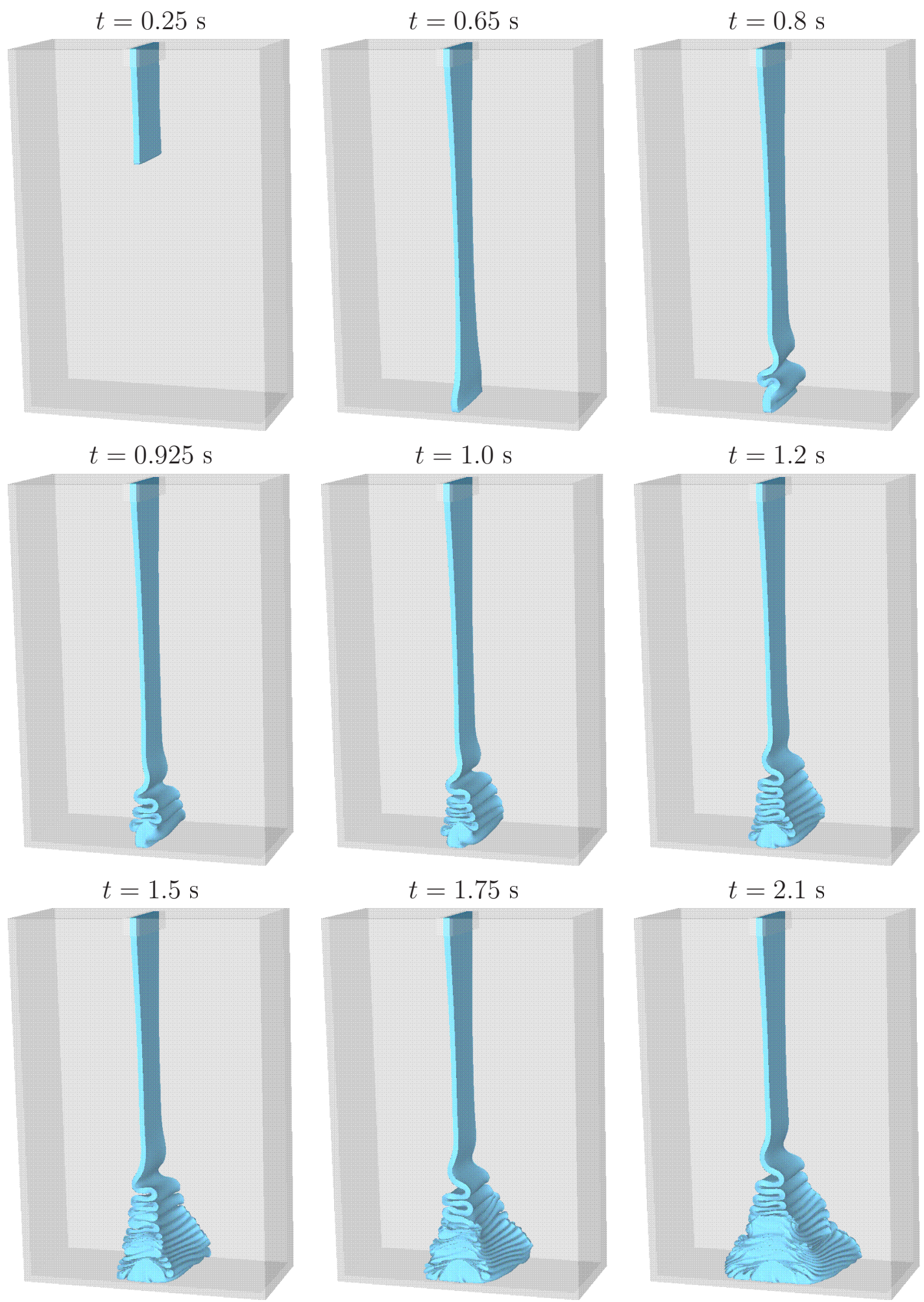

Figura 7.18: Ilustração da solução para o problema do jato planar em 3D para diferentes tempos. 


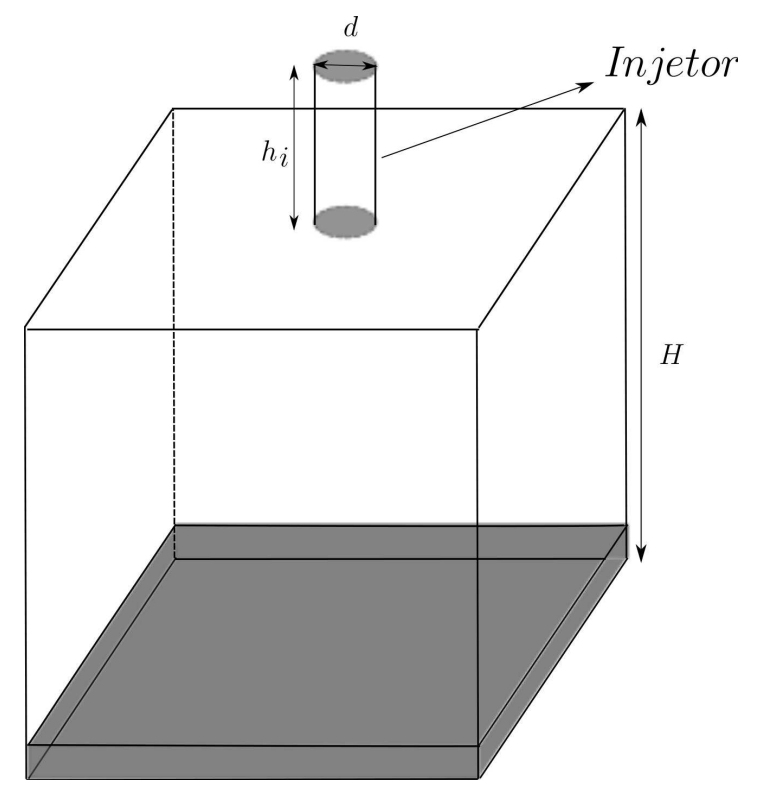

Figura 7.19: Esquematização para o problema do jato circular em $3 D$

$\diamond$ Altura do injetor: $h_{i}=0.2 m$;

$\diamond$ Relação $H / d=12.5$;

$\diamond$ Escala de velocidade: $U_{0}=1 \mathrm{~m} / \mathrm{s}$

$\diamond$ Escala de comprimento: $L_{0}=d=0.08 \mathrm{~m}$.

A solução numérica fornecida com a utilização do Esquema 1 é apresentada na Figura 7.20, onde verifica-se claramente a captura, com sucesso, do fenômeno de instabilidades físicas.

\section{Caso 2}

Nesse caso toma-se os seguintes dados:

$\diamond$ Domínio: $1 m \times 1 m \times 1.22 m$;

$\diamond$ Malha: $150 \times 150 \times 183 \Rightarrow \delta x=\delta y=\delta z=0.01$;

$\diamond$ Numero de Reynolds: $R_{e}=0.288$;

$\diamond$ Altura do injetor: $h_{i}=0.2 m$;

$\diamond$ relação $H / d=25$;

$\diamond$ Escala de velocidade: $U_{0}=1 \mathrm{~m} / \mathrm{s}$

$\diamond$ Escala de comprimento: $L_{0}=d=0.04 m$;

A simulação do jato circular oscilante referente a este caso é apresentada na Figura 7.21, onde compara-se a solução numérica com dados experimentais (ver [28]). Nota-se que o esquema é capaz de capturar o fenômeno, estando em concordância com tais dados experimentais. 


\section{Evolução temporal para o problema do jato circular 3D.}
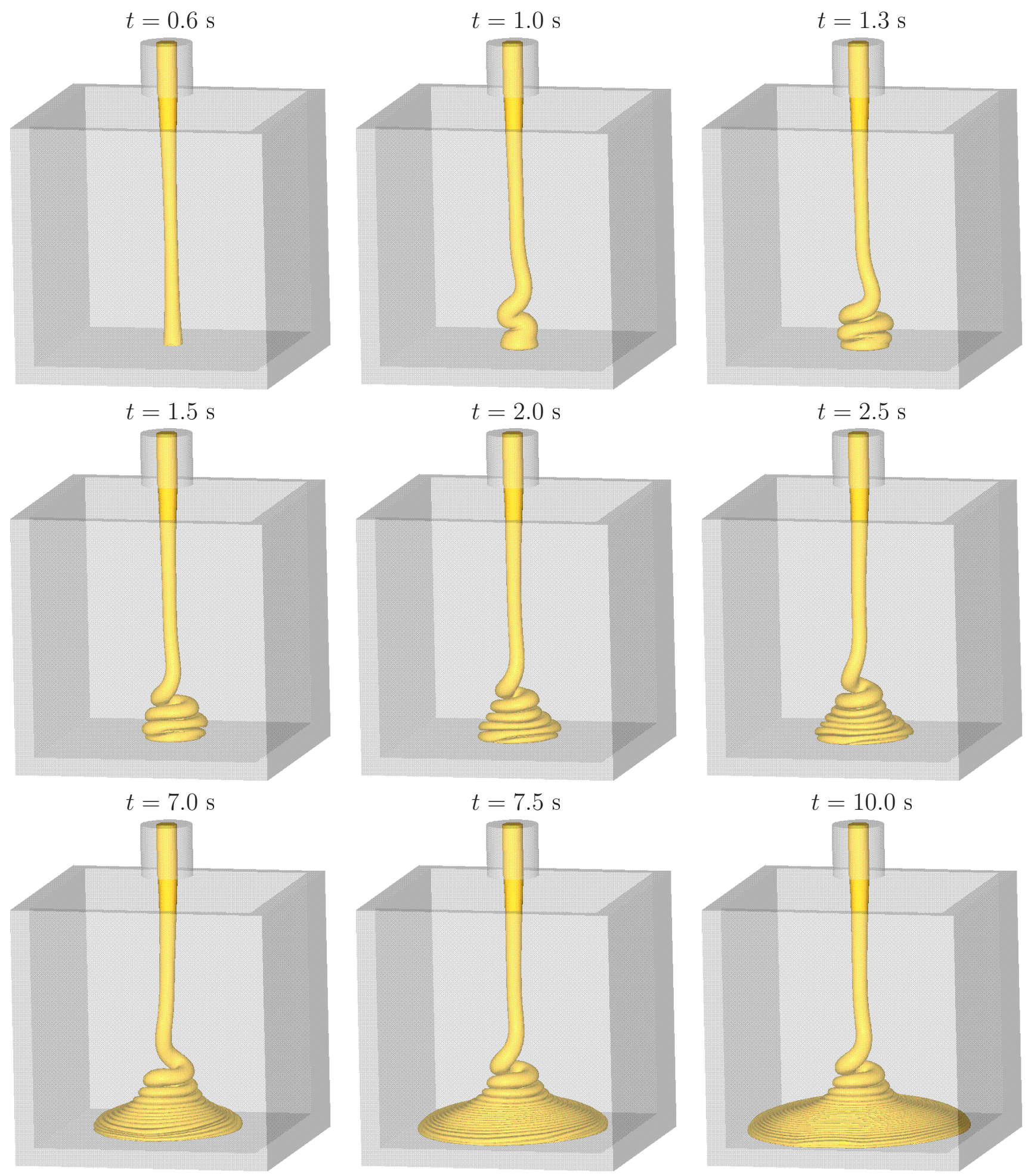

Figura 7.20: Ilustração da solução para o problema do jato circular em 3D para diferentes tempos-Caso 1. 
(a)

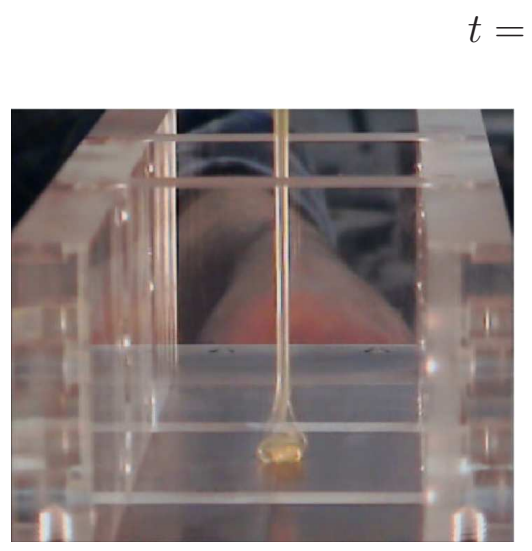

$t=1.4 \mathrm{~s}$
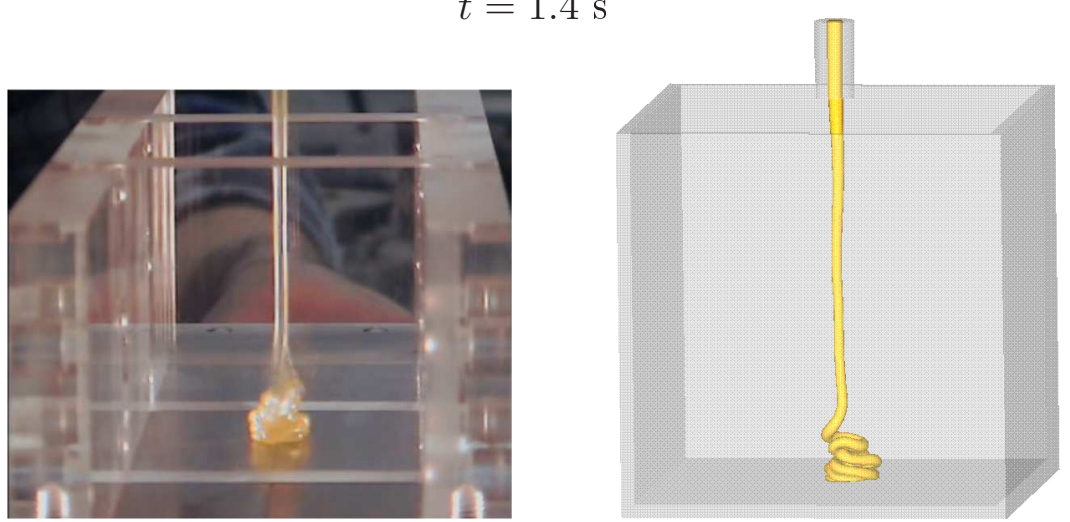

$t=3.6 \mathrm{~s}$
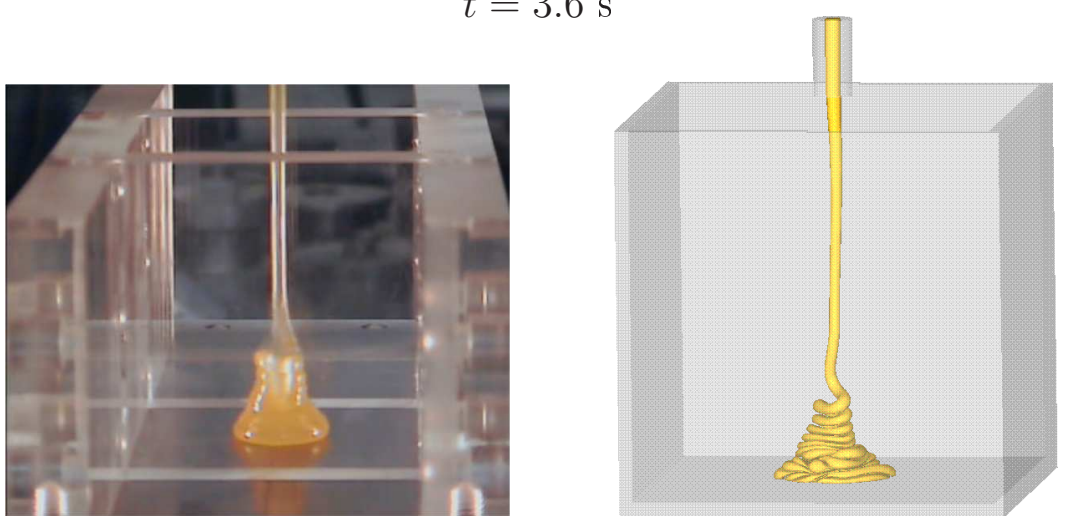

$$
t=9.6 \mathrm{~s}
$$
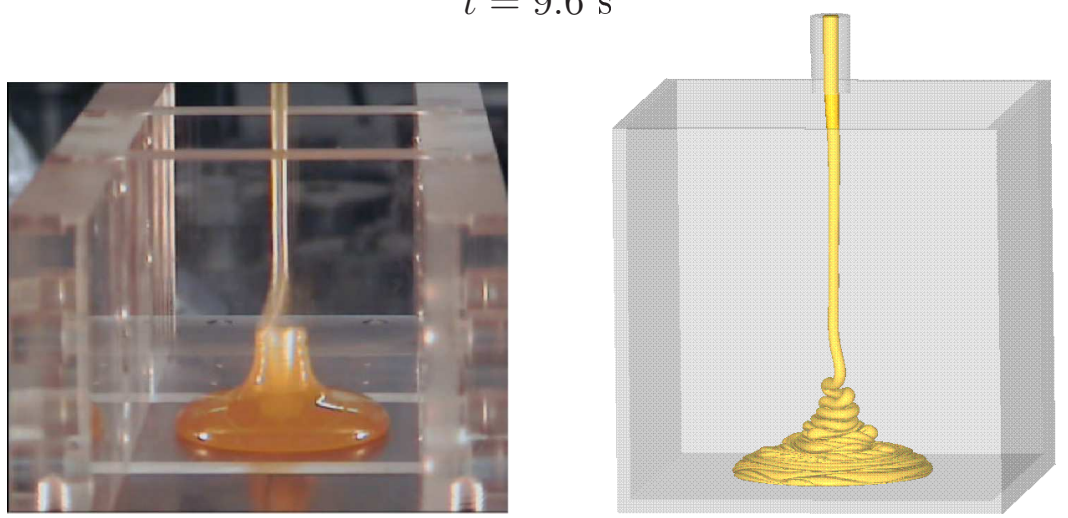

Figura 7.21: Ilustração do jato circular oscilante em 3D para diferentes tempos - Caso 2. (a) solução experimental; (b) solução numérica. 


\section{Ressalto hidráulico circular}

Outro problema interessante para testar o Esquema 1 é o fenômeno do ressalto hidráulico circular, discutido anteriormente neste capítulo para o caso $2.5 \mathrm{D}$, porém considerando agora as equações incompressíveis completas (3D) de Navier-Stokes. Apresentam-se dois casos, que diferem quanto ao numero de Reynolds. No caso 1 assume-se $R_{e}=250$ (laminar) e no caso 2 determina-se um problema mais instável, com $R_{e}=1000$ (transição para turbulência).

\section{Caso 1}

Para a simulação desse caso, os seguintes dados foram considerados:

$\diamond$ Domínio: $0.025 m \times 0.025 m \times 0.016 m$;

$\diamond$ Malha: $100 \times 100 \times 64 \Rightarrow \delta x=\delta y=\delta z=0.00025$;

$\diamond$ Diâmetro do injetor: $d=0.004 m$;

$\diamond$ Raio do injetor: $r_{i}=0.002 m$;

$\diamond$ Escala de velocidade: $U_{0}=0.375 \mathrm{~m} / \mathrm{s}$;

$\diamond$ Escala de comprimento: $L_{0}=2 d=0.008 \mathrm{~m}$.

Os resultados obtidos com o Esquema 1 e solução experimental de Rai [34] para o ressalto hidráulico circular em regime laminar estão apresentados nas Figuras 7.22 (b) e 7.22 (a), respectivamente. Como pode ser visto por essas figuras, o método numérico Freeflow3D equipado com o Esquema 1 capturou o mecanismo essencial desse escoamento com superfície livre.

(a)

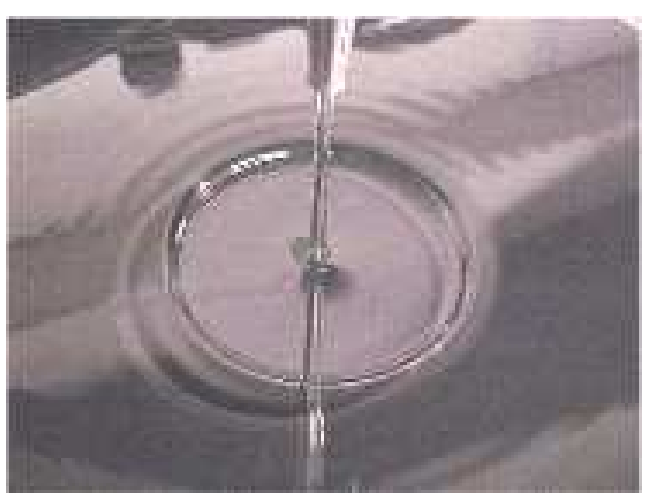

(b)

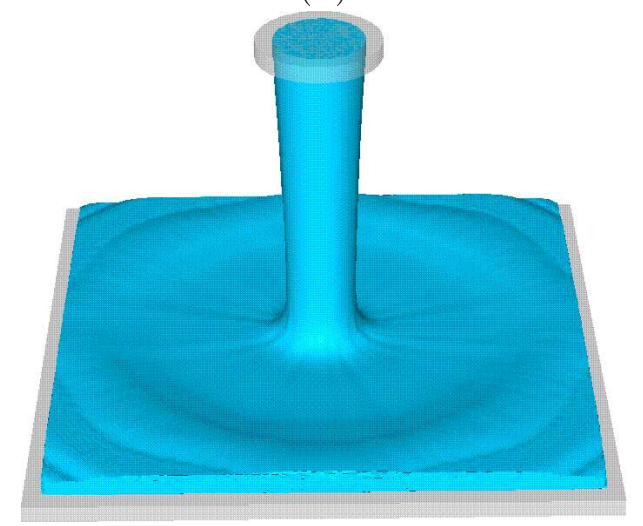

Figura 7.22: Ilustração do fenômeno do ressalto hidráulico circular- Caso 1. (a) solução experimental de Rai [34] ; (b) solução numérica.

Exibe-se a Figura 7.23 para ilustrar a evolução com o tempo do escoamento, mostrando a formação do ressalto hidráulico circular. 
Evolução temporal da solução numérica tomando $R_{e}=250$

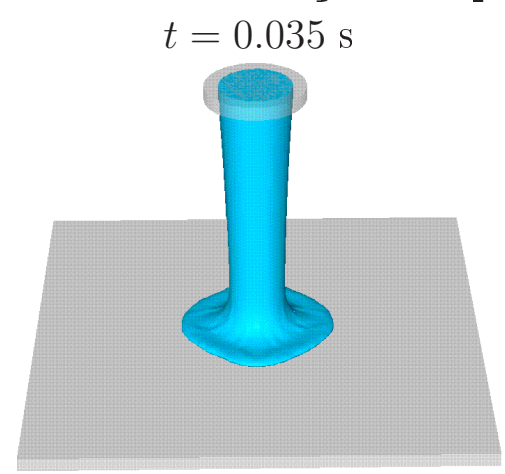

$t=0.115 \mathrm{~s}$

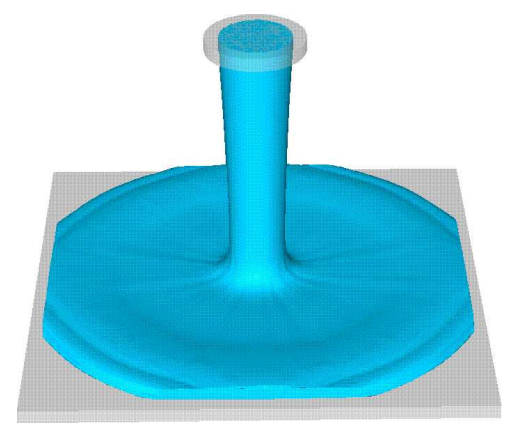

$t=0.07 \mathrm{~s}$

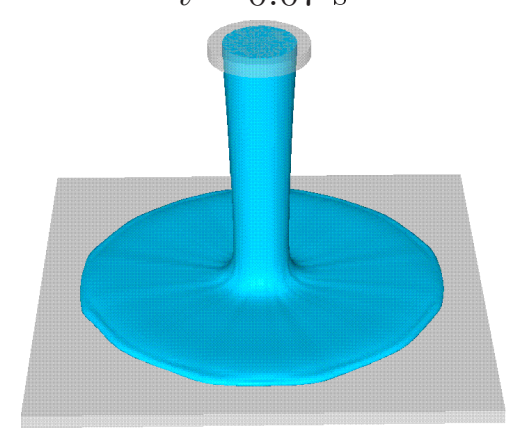

$t=0.15 \mathrm{~s}$

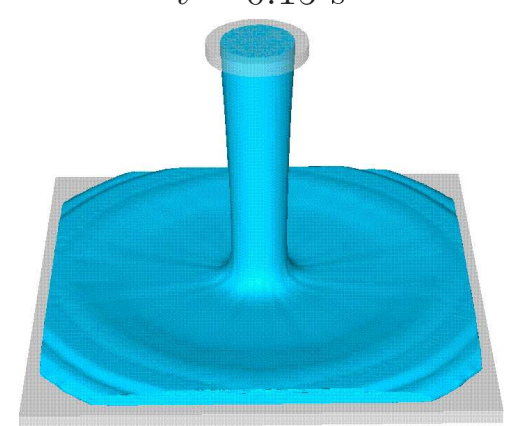

$t=0.1 \mathrm{~s}$

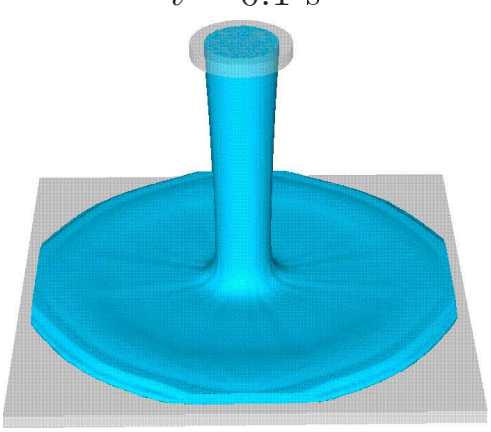

$t=0.175 \mathrm{~s}$

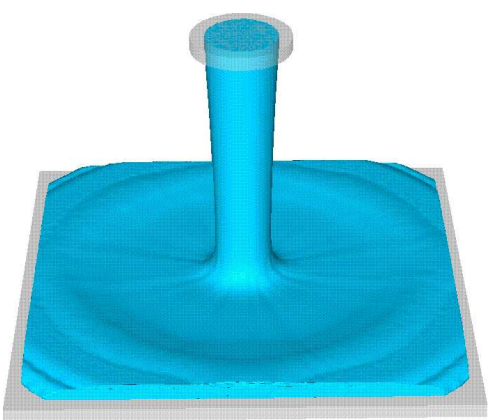

Figura 7.23: Ilustração da solução para o fenômeno do ressalto hidráulico em diferentes tempos para $R_{e}=250$ - Caso 1 .

\section{Caso 2}

Neste caso assume-se $R_{e}=1000$, o que torna mais evidente as instabilidades físicas após a região de ressalto. O seguintes dados foram empregados para a simulação:

$\diamond$ Domínio: $0.6 m \times 0.6 m \times 0.055 m$;

$\diamond$ Malha: $120 \times 120 \times 11 \Rightarrow \delta x=\delta y=\delta z=0.005$;

$\diamond$ Diâmetro do injetor: $d=0.05 m$;

$\diamond$ Raio do injetor: $r_{i}=0.025 \mathrm{~m}$;

$\diamond$ Escala de velocidade: $U_{0}=1 \mathrm{~m} / \mathrm{s}$;

$\diamond$ Escala de comprimento: $L_{0}=0.05 m$.

Na Figura 7.24 estão os resultados obtidos com o uso do Esquema 1 (ver 7.24 (b)), os quais são comparados com o experimento de Ellergard [9] (ver 7.24 (a)). Devido ao alto valor do número de Reynolds, a solução apresenta uma região de transição para turbulência após o ressalto. Por essas figuras, pode-se aferir que o esquema numérico conseguiu capturar a física correta do problema.

Exibe-se a Figura 7.25 para ilustrar o escoamento em vários tempos, onde é possível acompanhar a formação do ressalto hidráulico e a formação das instabilidades físicas após ocorrer o ressalto. 
(a)

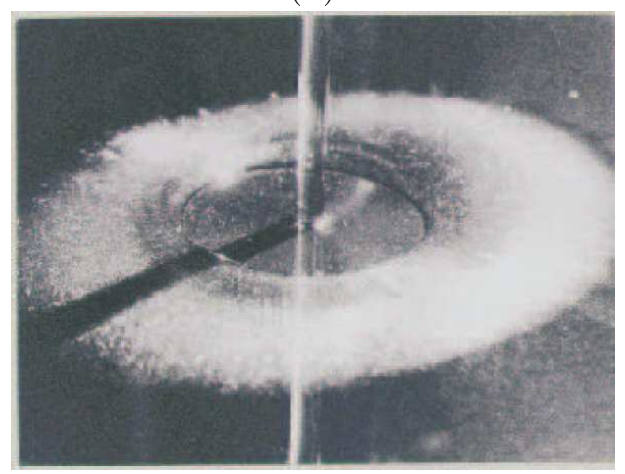

(b)

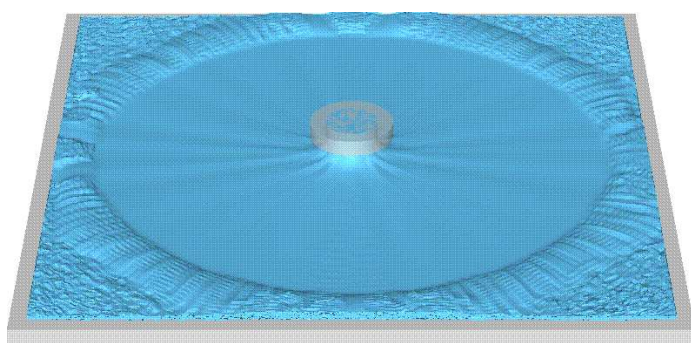

Figura 7.24: Ilustração do fenômeno do ressalto hidráulico circular- Caso 2. (a) solução experimental de Ellergard [9] ; (b) solução numérica.

Evolução temporal da solução numérica tomando $R_{e}=1000$

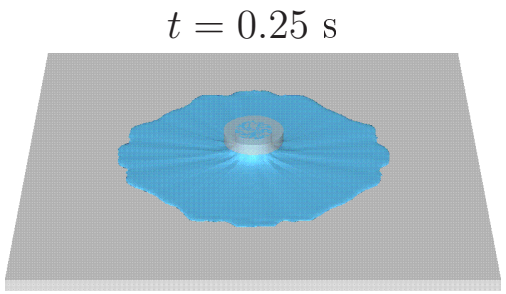

$t=0.8 \mathrm{~s}$

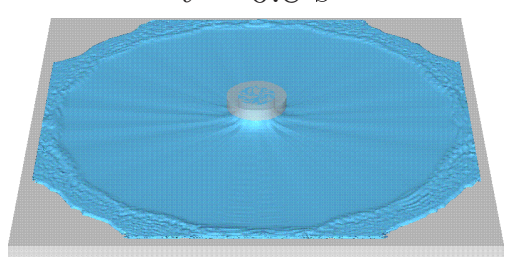

$t=0.5 \mathrm{~s}$

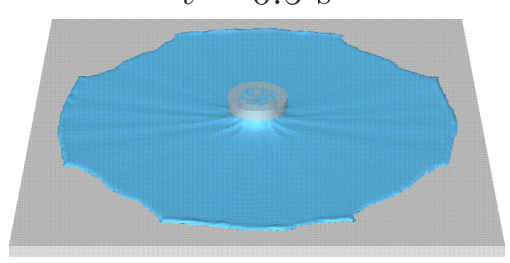

$t=0.95 \mathrm{~s}$

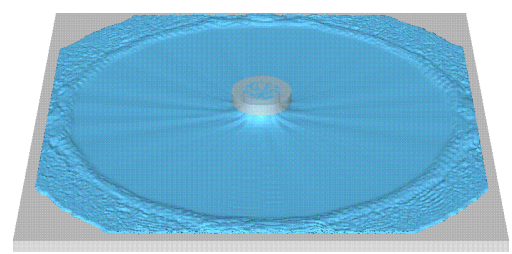

$t=0.65 \mathrm{~s}$

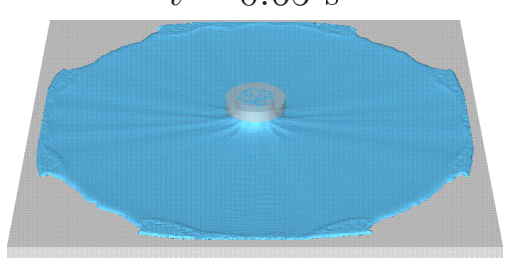

$t=1.15 \mathrm{~s}$

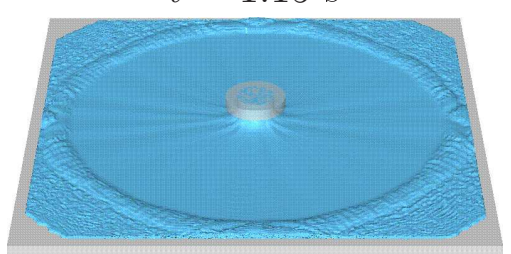

Figura 7.25: Ilustração da solução para o fenômeno do ressalto hidráulico em diferentes tempos para $R_{e}=1000$ - Caso 2 . 
CAPÍTULO

\section{8}

\section{Conclusões e Trabalhos Futuros}

Neste trabalho de mestrado, desenvolveu-se, analisou-se e implementou-se uma família de esquemas upwind dependentes de parâmetros, denominada FUS-RF, para aproximar termos convectivos (lineares e não lineares) de equações de conservação gerais. Em particular, seis esquemas dessa família denominados Esquema 1, Esquema 2, Esquema 3, Esquema 4, Esquema 5 e Esquema 6 foram testados. O Esquema 1 foi desenvolvido de maneira tradicional, tomando-se como base os critérios de estabilidade TVD e CBC, e os demais esquemas foram derivados via técnica de minimização mínimos quadrados ponderados.

Com o propósito de investigar o comportamento dos esquemas em leis de conservação hiperbólicas - e posterior classificação desses com respeito aos parâmetros livres - foi realizado uma bateria de testes numéricos variando os parâmetros $\beta$ para o Esquema 1 e $\epsilon$ para os demais esquemas. Investigou-se inicialmente o desempenho da família FUS-RF em problemas lineares (advecção de escalares) e não lineares (equação de Burgers 1D, águas rasas 1D/2D e equação de Euler 1D/2D); e comparações com os esquemas van Albada, EPUS e o WENO de quinta ordem foram realizadas. Verificou-se que, em todos esses testes, os seis esquemas apresentaram resultados confiáveis, com precisão igual (ou superior) à dos outros esquemas de referência, com destaque para os esquemas Esquema 1 e Esquema 5 que resolveram com sucesso as instabilidades de Rayleight Taylor (comportamento similar foi observado no problema dos quatro quadrantes). No geral, o Esquema 1 forneceu os melhores resultados com o uso do parâmetro livre $\beta=0.1545$; ao passo que os outros esquemas (de 2 a 6 ) forneceram resultados satisfatórios para vários valores do parâmetro $\epsilon$.

Tendo em vista os resultados obtidos em leis de conservação, o Esquema 1 com $\beta=0.1545$ foi então utilizado na simulação numérica de escoamentos incompressíveis com superfícies livres móveis, nos casos 2D, 2.5D e 3D. Em particular, o método numérico global (Freeflow atualizado com Esquema 1) foi capaz de simular com sucesso os problemas jet buckling, ressalto hidráulico 
circular e colapso de uma coluna de fluido,entre outros.

Em síntese, de um lado, o Esquema $1 \operatorname{com} \beta=0.1545$ é uma alternativa para a família de esquemas upwind por simular com sucesso problemas com descontinuidades e outros fenômenos em que os termos não lineares advectivos requerem atenção especial. Simulações em engenharia geralmente exigem (ao menos) segunda ordem de precisão espacial, e isto pode ser alcançado facilmente com este esquema. Por outro lado, a números moderados de Courant, recomenda-se também o uso dos esquemas Esquema 2, Esquema 3, Esquema 4, Esquema 5 e Esquema 6 com o parâmetro livre $\epsilon<10^{-1}$.

Para o futuro, pretende-se desenvolver novos esquemas para a família FUS-RF por meio do estudo/análise dos pesos $w_{1}$ e $w_{2}$ na técnica de mínimos quadrados ponderados. Objetiva-se ainda utilizar o Esquema 1 com $\beta=0.1545$ na simulação numérica de escoamentos viscoelásticos (com EDPs constitutivas tipo hiperbólicas) tridimensionais com superfície livres móveis. $\mathrm{E}$, finalmente, reunir-se-ão os resultados obtidos neste estudo num artigo para revista internacional. 
APÊNDICE

\section{Equações hiperbólicas na forma quase-linear}

Como visto no capítulo 2, as equações hiperbólicas podem ser escritas na forma quase-linear, ou seja,

$$
\frac{\partial \phi}{\partial t}+A_{1} \frac{\partial \phi}{\partial x_{1}}+A_{2} \frac{\partial \phi}{\partial x_{2}}+\cdots+A_{n} \frac{\partial \phi}{\partial x_{n}}=\zeta,
$$

onde $n$ é a dimensão, $\phi\left(x_{1}, x_{2}, \cdots, x_{n}, t\right): \mathbb{R}^{n} \times \mathbb{R} \rightarrow \mathbb{R}^{m}$ é um vetor das incógnitas do problema ( pressão, velocidade,... ) a ser determinado, e $A_{i_{m \times m}}$ uma matriz real, diagonalizável e com autovalores reais (ver LeVeque, [21]) e $\zeta$ são as fontes.

Neste apêndice, apresenta-se a transformação para a forma quase-linear das equações utilizadas neste trabalho.

\section{A.1 Equação de Advecção}

No caso $1 D$ considerando-se que não há termo fonte (ou seja, $\zeta=0$ ) tem-se:

$$
\frac{\partial \phi}{\partial t}+\frac{\partial F_{1}(\phi)}{\partial x_{1}}=0, \quad x_{1} \in\left[x_{L}, x_{R}\right], \quad t \in[0, T],
$$

onde $F_{1}(\phi)=u \phi$. Para escrever a equação em forma quasi-linear, calcula-se o Jacobiano $(J)$ do fluxo $F_{1}(\phi)$, ou seja

$$
A_{1}=J=\frac{\partial F_{1}(\phi)}{\partial \phi}=\frac{\partial \phi u}{\partial \phi}=u,
$$

e assim, a forma quasi-linear é

$$
\frac{\partial \phi}{\partial t}+u \frac{\partial \phi}{\partial x_{1}}=0, \quad x_{1} \in\left[x_{L}, x_{R}\right], \quad t \in[0, T] .
$$




\section{A.2 Equação de Burgers}

A equação de burgers no caso 1D é

$$
\frac{\partial \phi}{\partial t}+\frac{\partial F_{1}(\phi)}{\partial x_{1}}=\zeta, \quad x_{1} \in\left[x_{L}, x_{R}\right], \quad t \in[0, T],
$$

onde $F_{1}(\phi)=\frac{\phi^{2}}{2}$ e $\zeta$ é o termo fonte. Calculando o Jacobiano tem-se

$$
A_{1}=J=\frac{\partial F_{1}(\phi)}{\partial \phi}=\frac{\partial\left(\frac{\phi^{2}}{2}\right)}{\partial \phi}=\phi
$$

que resulta na forma quasi-linear

$$
\frac{\partial \phi}{\partial t}+\phi \frac{\partial \phi}{\partial x_{1}}=\zeta, \quad x_{1} \in\left[x_{L}, x_{R}\right], \quad t \in[0, T]
$$

\section{A.3 Equações de águas rasas}

No caso unidimensional, o sistema de águas rasas é

$$
\frac{\partial \phi}{\partial t}+\frac{\partial F_{1}(\phi)}{\partial x_{1}}=0
$$

no qual

$$
\phi=\left[\begin{array}{c}
h \\
h u
\end{array}\right]=\left[\begin{array}{l}
q_{1} \\
q_{2}
\end{array}\right], \quad F_{1}=\left[\begin{array}{c}
h u \\
h u^{2}+\frac{1}{2} g h^{2}
\end{array}\right]=\left[\begin{array}{c}
q_{2} \\
\frac{q_{2}^{2}}{q_{1}}+\frac{g q_{1}^{2}}{2}
\end{array}\right]=\left[\begin{array}{c}
A \\
B
\end{array}\right] .
$$

Calculando o Jacobiano tem-se

$$
A_{1}=J=\left[\begin{array}{ll}
\frac{\partial A}{\partial q_{1}} & \frac{\partial A}{\partial q_{2}} \\
\frac{\partial B}{\partial q_{1}} & \frac{\partial B}{\partial q_{2}}
\end{array}\right]=\left[\begin{array}{cc}
0 & 1 \\
\frac{-q_{2}^{2}}{q_{1}^{2}}+g q_{1} & \frac{2 q_{2}}{q_{1}}
\end{array}\right]=\left[\begin{array}{cc}
0 & 1 \\
-u^{2}+g h & 2 u
\end{array}\right]
$$

assim,

$$
\frac{\partial \phi}{\partial t}+\left[\begin{array}{cc}
0 & 1 \\
-u^{2}+g h & 2 u
\end{array}\right] \frac{\partial \phi}{\partial x_{1}}=0, \quad x_{1} \in\left[x_{L}, x_{R}\right], \quad t \in[0, T] .
$$

No caso 2D, o sistema é 


$$
\frac{\partial \phi}{\partial t}+\frac{\partial F_{1}(\phi)}{\partial x_{1}}+\frac{\partial F_{2}(\phi)}{\partial x_{2}}=0
$$

onde

$$
\begin{gathered}
\phi=\left[\begin{array}{c}
h \\
h u \\
h v
\end{array}\right]=\left[\begin{array}{l}
q_{1} \\
q_{2} \\
q_{3}
\end{array}\right], \quad F_{1}=\left[\begin{array}{c}
h u \\
h u^{2}+\frac{1}{2} g h^{2} \\
h u v
\end{array}\right]=\left[\begin{array}{c}
q_{2} \\
\frac{q_{2}^{2}}{q_{1}}+\frac{g q_{1}^{2}}{2} \\
\frac{q_{2} q_{3}}{q_{1}}
\end{array}\right], \\
F_{2}=\left[\begin{array}{c}
h v \\
h u v \\
h v^{2}+\frac{1}{2} g h^{2}
\end{array}\right]=\left[\begin{array}{c}
q_{3} \\
\frac{q_{2} q_{3}}{q_{1}} \\
\frac{q_{3}^{2}}{q_{1}}+\frac{g q_{1}^{2}}{2}
\end{array}\right]
\end{gathered}
$$

Calculando o Jacobiano tem-se

$$
A_{1}=J=\left[\begin{array}{ccc}
0 & 1 & 0 \\
\frac{-q_{2}^{2}}{q_{1}^{2}}+g q_{1} & \frac{2 q_{2}}{q_{1}} & 0 \\
\frac{-q_{3} q_{2}}{q_{1}^{2}} & \frac{q_{3}}{q_{1}} & \frac{q_{2}}{q_{1}}
\end{array}\right]=\left[\begin{array}{ccc}
0 & 1 & 0 \\
-u^{2}+g h & 2 u & 0 \\
-u v & v & u
\end{array}\right]
$$

e

$$
A_{2}=J=\left[\begin{array}{ccc}
0 & 0 & 1 \\
\frac{-q_{3} q_{2}}{q_{1}^{2}} & \frac{q_{3}}{q_{1}} & \frac{q_{2}}{q_{1}} \\
\frac{-q_{3}^{2}}{q_{1}^{2}}+g q_{1} & 0 & \frac{2 q_{3}}{q_{1}}
\end{array}\right]=\left[\begin{array}{ccc}
0 & 0 & 1 \\
-u v & v & u \\
-v^{2}+g h & 0 & 2 v
\end{array}\right]
$$

O sistema, na forma não conservativa, resulta em

$$
\frac{\partial \phi}{\partial t}+\left[\begin{array}{ccc}
0 & 1 & 0 \\
-u^{2}+g h & 2 u & 0 \\
-u v & v & u
\end{array}\right] \frac{\partial \phi}{\partial x_{1}}+\left[\begin{array}{ccc}
0 & 0 & 1 \\
-u v & v & u \\
-v^{2}+g h & 0 & 2 v
\end{array}\right] \frac{\partial \phi}{\partial x_{2}}=0 .
$$

\section{A.4 Equações de Euler}

No caso unidimensional, tem-se o sistema

$$
\frac{\partial \phi}{\partial t}+\frac{\partial F_{1}(\phi)}{\partial x_{1}}=\zeta
$$


em que

$$
\phi=\left[\begin{array}{c}
\rho \\
\rho u \\
E
\end{array}\right]=\left[\begin{array}{c}
q_{1} \\
q_{2} \\
q_{3}
\end{array}\right], \quad F_{1}=\left[\begin{array}{c}
\rho u \\
\rho u^{2}+p \\
u(E+p)
\end{array}\right]=\left[\begin{array}{c}
q_{2} \\
q_{3}(\gamma-1)+\frac{q_{2}^{2}}{q_{1}}\left(\frac{3}{2}-\frac{\gamma}{2}\right) \\
\frac{\gamma q_{3} q_{2}}{q_{1}}+\frac{1}{2} \frac{q_{2}^{3}(1-\gamma)}{q_{1}^{2}}
\end{array}\right],
$$

em que $p=(\gamma-1)\left(E-\frac{1}{2} \rho u^{2}\right)$

O Jacobiano correspondente é

$$
\begin{aligned}
A_{1}=J & =\left[\begin{array}{ccc}
0 & 1 & 0 \\
\frac{-q_{2}{ }^{2}}{q_{1}{ }^{2}}\left(\frac{3}{2}-\frac{\gamma}{2}\right) & \frac{2 q_{2}}{q_{1}}\left(\frac{3}{2}-\frac{\gamma}{2}\right) & (\gamma-1) \\
\frac{-\gamma q_{3} q_{2}}{q_{1}{ }^{2}}-\frac{q_{2}^{3}}{q_{1}^{3}}(1-\gamma) & \frac{\gamma q_{3}}{q_{1}}+\frac{3}{2} \frac{q_{2}^{2}}{q_{1}^{2}}(1-\gamma) & \frac{\gamma q_{2}}{q_{1}}
\end{array}\right] \\
= & {\left[\begin{array}{ccc}
0 & 1 & 0 \\
\frac{1}{2}(\gamma-3) u^{2} & (3-\gamma) u & \gamma-1 \\
\frac{-\gamma E u}{\rho}-u^{3}(1-\gamma) & \frac{\gamma E}{\rho}+\frac{3}{2} u^{2}(1-\gamma) & \gamma u
\end{array}\right], }
\end{aligned}
$$

assim,

$$
\frac{\partial \phi}{\partial t}+\left[\begin{array}{ccc}
0 & 1 & 0 \\
\frac{1}{2}(\gamma-3) u^{2} & (3-\gamma) u & \gamma-1 \\
\frac{-\gamma E u}{\rho}-u^{3}(1-\gamma) & \frac{\gamma E}{\rho}+\frac{3}{2} u^{2}(1-\gamma) & \gamma u
\end{array}\right] \frac{\partial \phi}{\partial x_{1}}=\zeta, \quad x_{1} \in\left[x_{L}, x_{R}\right], \quad t \in[0, T] .
$$

No caso bidimensional, o sistema de Euler é

$$
\frac{\partial \phi}{\partial t}+\frac{\partial F_{1}(\phi)}{\partial x_{1}}+\frac{\partial F_{2}(\phi)}{\partial x_{2}}=\zeta,
$$


em que

$$
\begin{aligned}
& \phi=\left[\begin{array}{c}
\rho \\
\rho u \\
\rho v \\
E
\end{array}\right]=\left[\begin{array}{c}
q_{1} \\
q_{2} \\
q_{3} \\
q_{4}
\end{array}\right], \quad F_{1}=\left[\begin{array}{c}
\rho u \\
\rho u^{2}+p \\
\rho u v \\
u(E+p)
\end{array}\right]=\left[\begin{array}{c}
q_{2} \\
\frac{q_{2}^{2}}{q_{1}}+(\gamma-1)\left(q_{4}-\frac{q_{2}^{2}}{2 q_{1}}-\frac{q_{3}^{2}}{2 q_{1}}\right) \\
\frac{q_{2} q_{3}}{q_{1}} \\
\frac{\gamma q_{4} q_{2}}{q_{1}}-(\gamma-1)\left(\frac{q_{2}^{3}}{2 q_{1}^{2}}+\frac{q_{2} q_{3}^{2}}{2 q_{1}^{2}}\right)
\end{array}\right], \\
& F_{2}=\left[\begin{array}{c}
\rho v \\
\rho u v \\
\rho v^{2}+p \\
v(E+p)
\end{array}\right]=\left[\begin{array}{c}
q_{3} \\
\frac{q_{2} q_{3}}{q_{1}} \\
\frac{q_{3}^{2}}{q_{1}}+(\gamma-1)\left(q_{4}-\frac{q_{3}^{2}}{2 q_{1}}-\frac{q_{2}^{2}}{2 q_{1}}\right) \\
\frac{\gamma q_{4} q_{3}}{q_{1}}-(\gamma-1)\left(\frac{q_{3}^{3}}{2 q_{1}^{2}}+\frac{q_{3} q_{2}^{2}}{2 q_{1}^{2}}\right)
\end{array}\right]
\end{aligned}
$$

Calculando o Jacobiano tem-se

$$
\begin{aligned}
& A_{1}=\left[\begin{array}{cccc}
0 & 1 & 0 & 0 \\
\frac{-q_{2}^{2}}{q_{1}^{2}}+(\gamma-1)\left(\frac{q_{2}^{2}}{2 q_{1}^{2}}+\frac{q_{3}^{2}}{2 q_{1}^{2}}\right) & \frac{2 q_{2}}{q_{1}}-(\gamma-1) \frac{q_{2}}{q_{1}} & -(\gamma-1) \frac{q_{3}}{q_{1}} & \gamma-1 \\
-\frac{q_{2} q_{3}}{q_{1}^{2}} & \frac{q_{3}}{q_{1}} & \frac{q_{2}}{q_{1}} & 0 \\
\frac{-\gamma q_{4} q_{2}}{q_{1}^{2}}-(\gamma-1)\left(-\frac{q_{2}^{3}}{q_{1}^{3}}-\frac{q_{2} q_{3}^{2}}{q_{1}^{3}}\right) & \frac{\gamma q_{4}}{q_{1}}-(\gamma-1)\left(\frac{3 q_{2}^{2}}{2 q_{1}^{2}}+\frac{q_{3}^{2}}{2 q_{1}^{2}}\right) & -(\gamma-1) \frac{q_{2} q_{3}}{q_{1}^{2}} & \frac{\gamma q_{2}}{q_{1}}
\end{array}\right] \\
& =\left[\begin{array}{cccc}
0 & 1 & 0 & 0 \\
-u^{2}+(\gamma-1)\left(\frac{u^{2}}{2}+\frac{v^{2}}{2}\right) & (3-\gamma) u & -v(\gamma-1) & (\gamma-1) \\
-u v & v & u & 0 \\
\frac{-\gamma E u}{\rho}+(\gamma-1)\left(u^{3}+u v^{2}\right) & \frac{\gamma E}{\rho}-(\gamma-1)\left(\frac{3 u^{2}}{2}+\frac{v^{2}}{2}\right) & -(\gamma-1) u v & \gamma u
\end{array}\right],
\end{aligned}
$$




$$
\begin{aligned}
& A_{2}=\left[\begin{array}{cccc}
0 & 0 & 1 & 0 \\
-\frac{q_{2} q_{3}}{q_{1}^{2}} & \frac{q_{3}}{q_{1}} & \frac{q_{2}}{q_{1}} & 0 \\
\frac{-q_{3}^{2}}{q_{1}^{2}}+(\gamma-1)\left(\frac{q_{3}^{2}}{2 q_{1}^{2}}+\frac{q_{2}^{2}}{2 q_{1}^{2}}\right) & -(\gamma-1) \frac{q_{2}}{q_{1}} & \frac{2 q_{3}}{q_{1}}-(\gamma-1) \frac{q_{3}}{q_{1}} & \gamma-1 \\
\frac{-\gamma q_{4}}{q_{1}}-(\gamma-1)\left(\frac{q_{3}^{3}}{2 q_{1}^{2}}+\frac{q_{3} q_{2}^{2}}{2 q_{1}^{2}}\right) & -(\gamma-1) \frac{q_{2} q_{3}}{q_{1}^{2}} & \frac{\gamma q_{4}}{q_{1}}-(\gamma-1)\left(\frac{3 q_{3}^{2}}{2 q_{1}^{2}}+\frac{q_{2}^{2}}{2 q_{1}^{2}}\right) & \frac{\gamma q_{3}}{q_{1}}
\end{array}\right] \\
& =\left[\begin{array}{cccc}
0 & 0 & 1 & 0 \\
-u v & v & u & 0 \\
-v^{2}+(\gamma-1)\left(\frac{u^{2}}{2}+\frac{v^{2}}{2}\right) & -u(\gamma-1) & (3-\gamma) v & (\gamma-1) \\
\frac{-\gamma E v}{\rho}+(\gamma-1)\left(v^{3}+v u^{2}\right) & -(\gamma-1) u v & \frac{\gamma E}{\rho}-(\gamma-1)\left(\frac{3 v^{2}}{2}+\frac{u^{2}}{2}\right) & \gamma v
\end{array}\right] .
\end{aligned}
$$

O sistema, na forma não conservativa, resulta em

$$
\frac{\partial \phi}{\partial t}+\left[\begin{array}{cccc}
0 & 1 & 0 & 0 \\
-u^{2}+(\gamma-1)\left(\frac{u^{2}}{2}+\frac{v^{2}}{2}\right) & (3-\gamma) u & -v(\gamma-1) & (\gamma-1) \\
-u v & v & u & 0 \\
\frac{-\gamma E u}{\rho}+(\gamma-1)\left(u^{3}+u v^{2}\right) & \frac{\gamma E}{\rho}-(\gamma-1)\left(\frac{3 u^{2}}{2}+\frac{v^{2}}{2}\right) & -(\gamma-1) u v & \gamma u
\end{array}\right]{\frac{\partial \phi}{\partial x_{1}}+}^{-}
$$

$$
\left[\begin{array}{cccc}
0 & 0 & 1 & 0 \\
-u v & v & u & 0 \\
-v^{2}+(\gamma-1)\left(\frac{u^{2}}{2}+\frac{v^{2}}{2}\right) & -u(\gamma-1) & (3-\gamma) v & (\gamma-1) \\
\frac{-\gamma E v}{\rho}+(\gamma-1)\left(v^{3}+v u^{2}\right) & -(\gamma-1) u v & \frac{\gamma E}{\rho}-(\gamma-1)\left(\frac{3 v^{2}}{2}+\frac{u^{2}}{2}\right) & \gamma v
\end{array}\right] \frac{\partial \phi}{\partial x_{2}}=\zeta
$$




\section{Discretização dos termos convectivos}

Neste apêndice apresenta-se a discretização computacional dos termos convectivos para as equações de Navier-Stokes. Assumindo o eixo $x$ (para os outros eixos a aproximação é análoga), tem-se as faces $f$ em $\left(i+\frac{1}{2}, j, k\right)$ e $g$ em $\left(i-\frac{1}{2}, j, k\right)$. Tomando a face $f$ (a face $g$ é construída de forma similar) constrói-se a seguinte aproximação:

$$
\begin{aligned}
\left.\left(\frac{\partial(u u)}{\partial x}+\frac{\partial(u v)}{\partial y}+\frac{\partial(u w)}{\partial z}\right)\right|_{i+\frac{1}{2}, j, k} \approx & \frac{\bar{u}_{1} u_{i+1, j, k}-\bar{u}_{2} u_{i, j, k}}{\delta x}+\frac{\bar{v}_{1} u_{i+\frac{1}{2}, j+\frac{1}{2}, k}-\bar{v}_{2} u_{i+\frac{1}{2}, j-\frac{1}{2}, k}}{\delta y} \\
& +\frac{\bar{w}_{1} u_{i+\frac{1}{2}, j, k+\frac{1}{2}}-\bar{w}_{2} u_{i+\frac{1}{2}, j, k-\frac{1}{2}}}{\delta z}
\end{aligned}
$$

Os valores das velocidades convectivas $\bar{u}_{1}, \bar{u}_{2}, \bar{v}_{1}, \bar{v}_{2}, \bar{w}_{1}$ e $\bar{w}_{2}$ em (B.1) são aproximados por médias. Por exemplo, os valores $\bar{v}_{1}$ e $\bar{v}_{2}$ são aproximados por

$$
\bar{v}_{1}=\bar{v}_{i+\frac{1}{2}, j+\frac{1}{2}, k} \approx 0.5\left(v_{i+1, j+\frac{1}{2}, k}+v_{i, j+\frac{1}{2}, k}\right)
$$

e

$$
\bar{v}_{2}=\bar{v}_{i+\frac{1}{2}, j-\frac{1}{2}, k} \approx 0.5\left(v_{i+1, j-\frac{1}{2}, k}+v_{i, j-\frac{1}{2}, k}\right) .
$$

Os demais termos da equação B.1 são aproximados pelos esquemas Upwind, observando o sinal da velocidade de convecção. Desta forma, em NV definem-se unicamente os pontos $D, U$ e $R$.

(1) Aproximações para $u_{i+1, j, k}$ : 
- Quando $\bar{u}_{1}=u_{i+1, j, k}>0$ tem-se $\hat{u}_{i+\frac{1}{2}, j, k}=\frac{u_{i+\frac{1}{2}, j, k}-u_{i-\frac{1}{2}, j, k}}{u_{i+\frac{3}{2}, j, k}-u_{i-\frac{1}{2}, j, k}}$ e assim

$$
u_{i+1, j, k}=\left\{\begin{array}{lll}
u_{R}+\left(u_{D}-u_{R}\right) \phi_{f}\left(\hat{u}_{U}\right), & \text { se } & \hat{u}_{U} \in[0,1] \\
u_{u}, & \text { se } & \hat{u}_{U} \notin[0,1]
\end{array}\right.
$$

onde

$$
D=\left(i+\frac{3}{2}, j, k\right), \quad U=\left(i+\frac{1}{2}, j, k\right), \quad R=\left(i-\frac{1}{2}, j, k\right)
$$

- Quando $\bar{u}_{1}=u_{i+1, j, k}<0$ tem-se $\hat{u}_{i+\frac{3}{2}, j, k}=\frac{u_{i+\frac{3}{2}, j, k}-u_{i+\frac{5}{2}, j, k}}{u_{i+\frac{1}{2}, j, k}-u_{i+\frac{5}{2}, j, k}} \operatorname{logo}$

$$
u_{i+1, j, k}=\left\{\begin{array}{lll}
u_{R}+\left(u_{D}-u_{R}\right) \hat{\phi}_{f}\left(\hat{u}_{U}\right), & \text { se } & \hat{u}_{U} \in[0,1] \\
u_{u}, & \text { se } & \hat{u}_{U} \notin[0,1]
\end{array}\right.
$$

na qual

$$
D=\left(i+\frac{1}{2}, j, k\right), \quad U=\left(i+\frac{3}{2}, j, k\right), \quad R=\left(i+\frac{5}{2}, j, k\right)
$$

(2) Aproximações para $u_{i, j, k}$ :

- Quando $\bar{u}_{2}=u_{i, j, k}>0$ tem-se $\hat{u}_{i-\frac{1}{2}, j, k}=\frac{u_{i-\frac{1}{2}, j, k}-u_{i-\frac{3}{2}, j, k}}{u_{i+\frac{1}{2}, j, k}-u_{i-\frac{3}{2}, j, k}}$ e assim

$$
u_{i, j, k}=\left\{\begin{array}{lll}
u_{R}+\left(u_{D}-u_{R}\right) \hat{\phi}_{f}\left(\hat{u}_{U}\right), & \text { se } & \hat{u}_{U} \in[0,1] \\
u_{u}, & \text { se } & \hat{u}_{U} \notin[0,1]
\end{array}\right.
$$

onde

$$
D=\left(i+\frac{1}{2}, j, k\right), \quad U=\left(i-\frac{1}{2}, j, k\right), \quad R=\left(i-\frac{3}{2}, j, k\right) \text {; }
$$

- Quando $\bar{u}_{2}=u_{i, j, k}<0$ tem-se $\hat{u}_{i+\frac{1}{2}, j, k}=\frac{u_{i+\frac{1}{2}, j, k}-u_{i+\frac{3}{2}, j, k}}{u_{i-\frac{1}{2}, j, k}-u_{i+\frac{3}{2}, j, k}} \operatorname{logo}$

$$
u_{i, j, k}=\left\{\begin{array}{lll}
u_{R}+\left(u_{D}-u_{R}\right) \hat{\phi}_{f}\left(\hat{u}_{U}\right), & \text { se } & \hat{u}_{U} \in[0,1] \\
u_{u}, & \text { se } & \hat{u}_{U} \notin[0,1]
\end{array}\right.
$$

na qual

$$
D=\left(i-\frac{1}{2}, j, k\right), \quad U=\left(i+\frac{1}{2}, j, k\right), \quad R=\left(i+\frac{3}{2}, j, k\right) \text {; }
$$

(3) Aproximações para $u_{i+\frac{1}{2}, j+\frac{1}{2}, k}$ : 
- Quando $\bar{v}_{1}=v_{i+\frac{1}{2}, j+\frac{1}{2}, k}>0$ tem-se $\hat{u}_{i+\frac{1}{2}, j, k}=\frac{u_{i+\frac{1}{2}, j, k}-u_{i+\frac{1}{2}, j-1, k}}{u_{i+\frac{1}{2}, j+1, k}-u_{i+\frac{1}{2}, j-1, k}}$ e assim

$$
u_{i+\frac{1}{2}, j+\frac{1}{2}, k}=\left\{\begin{array}{lll}
u_{R}+\left(u_{D}-u_{R}\right) \hat{\phi}_{f}\left(\hat{u}_{U}\right), & \text { se } & \hat{u}_{U} \in[0,1] \\
u_{u}, & \text { se } & \hat{u}_{U} \notin[0,1]
\end{array}\right.
$$

onde

$$
D=\left(i+\frac{1}{2}, j+1, k\right), \quad U=\left(i+\frac{1}{2}, j, k\right), \quad R=\left(i+\frac{1}{2}, j-1, k\right)
$$

- Quando $\bar{v}_{1}=v_{i+\frac{1}{2}, j+\frac{1}{2}, k}<0$ tem-se $\hat{u}_{i+\frac{1}{2}, j+1, k}=\frac{u_{i+\frac{1}{2}, j+1, k}-u_{i+\frac{1}{2}, j+2, k}}{u_{i+\frac{1}{2}, j, k}-u_{i+\frac{1}{2}, j+2, k}} \operatorname{logo}$

$$
u_{i+\frac{1}{2}, j+\frac{1}{2}, k}=\left\{\begin{array}{lll}
u_{R}+\left(u_{D}-u_{R}\right) \hat{\phi}_{f}\left(\hat{u}_{U}\right), & \text { se } & \hat{u}_{U} \in[0,1] \\
u_{u}, & \text { se } & \hat{u}_{U} \notin[0,1]
\end{array}\right.
$$

na qual

$$
D=\left(i+\frac{1}{2}, j, k\right), \quad U=\left(i+\frac{1}{2}, j+1, k\right), \quad R=\left(i+\frac{1}{2}, j+2, k\right)
$$

(4) Aproximações para $u_{i+\frac{1}{2}, j-\frac{1}{2}, k}$ :

- Quando $\bar{v}_{2}=v_{i+\frac{1}{2}, j-\frac{1}{2}, k}>0$ tem-se $\hat{u}_{i+\frac{1}{2}, j-1, k}=\frac{u_{i+\frac{1}{2}, j-1, k}-u_{i+\frac{1}{2}, j-2, k}}{u_{i+\frac{1}{2}, j, k}-u_{i+\frac{1}{2}, j-2, k}}$ e assim

$$
u_{i+\frac{1}{2}, j-\frac{1}{2}, k}=\left\{\begin{array}{lll}
u_{R}+\left(u_{D}-u_{R}\right) \hat{\phi}_{f}\left(\hat{u}_{U}\right), & \text { se } & \hat{u}_{U} \in[0,1], \\
u_{u}, & \text { se } & \hat{u}_{U} \notin[0,1]
\end{array}\right.
$$

onde

$$
D=\left(i+\frac{1}{2}, j, k\right), \quad U=\left(i+\frac{1}{2}, j-1, k\right), \quad R=\left(i+\frac{1}{2}, j-2, k\right)
$$

- Quando $\bar{v}_{2}=v_{i+\frac{1}{2}, j-\frac{1}{2}, k}<0$ tem-se $\hat{u}_{i+\frac{1}{2}, j, k}=\frac{u_{i+\frac{1}{2}, j, k}-u_{i+\frac{1}{2}, j+1, k}}{u_{i+\frac{1}{2}, j-1, k}-u_{i+\frac{1}{2}, j+1, k}} \operatorname{logo}$

$$
u_{i+\frac{1}{2}, j-\frac{1}{2}, k}=\left\{\begin{array}{lll}
u_{R}+\left(u_{D}-u_{R}\right) \hat{\phi}_{f}\left(\hat{u}_{U}\right), & \text { se } & \hat{u}_{U} \in[0,1], \\
u_{u}, & \text { se } & \hat{u}_{U} \notin[0,1]
\end{array}\right.
$$

na qual

$$
D=\left(i+\frac{1}{2}, j-1, k\right), \quad U=\left(i+\frac{1}{2}, j, k\right), \quad R=\left(i+\frac{1}{2}, j+1, k\right) ;
$$

(5) Aproximações para $u_{i+\frac{1}{2}, j, k+\frac{1}{2}}$ : 
- Quando $\bar{w}_{1}=w_{i+\frac{1}{2}, j, k+\frac{1}{2}}>0$ tem-se $\hat{u}_{i+\frac{1}{2}, j, k}=\frac{u_{i+\frac{1}{2}, j, k}-u_{i+\frac{1}{2}, j, k-1}}{u_{i+\frac{1}{2}, j, k+1}-u_{i+\frac{1}{2}, j, k-1}}$ e assim

$$
u_{i+\frac{1}{2}, j, k+\frac{1}{2}}=\left\{\begin{array}{lll}
u_{R}+\left(u_{D}-u_{R}\right) \hat{\phi}_{f}\left(\hat{u}_{U}\right), & \text { se } & \hat{u}_{U} \in[0,1], \\
u_{u}, & \text { se } & \hat{u}_{U} \notin[0,1]
\end{array}\right.
$$

onde

$$
D=\left(i+\frac{1}{2}, j, k+1\right), \quad U=\left(i+\frac{1}{2}, j, k\right), \quad R=\left(i+\frac{1}{2}, j, k-1\right) ;
$$

- Quando $\bar{w}_{1}=w_{i+\frac{1}{2}, j, k+\frac{1}{2}}<0$ tem-se $\hat{u}_{i+\frac{1}{2}, j, k+1}=\frac{u_{i+\frac{1}{2}, j, k+1}-u_{i+\frac{1}{2}, j, k+2}}{u_{i+\frac{1}{2}, j, k}-u_{i+\frac{1}{2}, j, k+2}} \operatorname{logo}$

$$
u_{i+\frac{1}{2}, j, k+\frac{1}{2}}=\left\{\begin{array}{lll}
u_{R}+\left(u_{D}-u_{R}\right) \hat{\phi}_{f}\left(\hat{u}_{U}\right), & \text { se } & \hat{u}_{U} \in[0,1] \\
u_{u}, & \text { se } & \hat{u}_{U} \notin[0,1]
\end{array}\right.
$$

na qual

$$
D=\left(i+\frac{1}{2}, j, k\right), \quad U=\left(i+\frac{1}{2}, j, k+1\right), \quad R=\left(i+\frac{1}{2}, j, k+2\right) ;
$$

(6) Aproximações para $u_{i+\frac{1}{2}, j, k-\frac{1}{2}}$ :

- Quando $\bar{w}_{2}=w_{i+\frac{1}{2}, j, k-\frac{1}{2}}>0$ tem-se $\hat{u}_{i+\frac{1}{2}, j, k-1}=\frac{u_{i+\frac{1}{2}, j, k-1}-u_{i+\frac{1}{2}, j, k-2}}{u_{i+\frac{1}{2}, j, k}-u_{i+\frac{1}{2}, j, k-2}}$ e assim

$$
u_{i+\frac{1}{2}, j, k-\frac{1}{2}}=\left\{\begin{array}{lll}
u_{R}+\left(u_{D}-u_{R}\right) \hat{\phi}_{f}\left(\hat{u}_{U}\right), & \text { se } & \hat{u}_{U} \in[0,1], \\
u_{u}, & \text { se } & \hat{u}_{U} \notin[0,1]
\end{array}\right.
$$

onde

$$
D=\left(i+\frac{1}{2}, j, k\right), \quad U=\left(i+\frac{1}{2}, j, k-1\right), \quad R=\left(i+\frac{1}{2}, j, k-2\right)
$$

- Quando $\bar{w}_{2}=w_{i+\frac{1}{2}, j, k-\frac{1}{2}}<0$ tem-se $\hat{u}_{i+\frac{1}{2}, j, k}=\frac{u_{i+\frac{1}{2}, j, k}-u_{i+\frac{1}{2}, j, k+1}}{u_{i+\frac{1}{2}, j, k-1}-u_{i+\frac{1}{2}, j, k+1}} \operatorname{logo}$

$$
u_{i+\frac{1}{2}, j, k-\frac{1}{2}}=\left\{\begin{array}{lll}
u_{R}+\left(u_{D}-u_{R}\right) \hat{\phi}_{f}\left(\hat{u}_{U}\right), & \text { se } & \hat{u}_{U} \in[0,1], \\
u_{u}, & \text { se } & \hat{u}_{U} \notin[0,1]
\end{array}\right.
$$

na qual

$$
D=\left(i+\frac{1}{2}, j, k-1\right), \quad U=\left(i+\frac{1}{2}, j, k\right), \quad R=\left(i+\frac{1}{2}, j, k+1\right) ;
$$




\section{Referências Bibliográficas}

[1] P. J. Alves, M. A. Oliveira and F.T. Pinho. A convergent and universally bounded interpolation scheme for the treatment of advection. International Journal for Numerical Methods in Fluids, 41(1):47-75, 2003.

[2] C. Berthon. Stability of the MUSCL schemes for the Euler equations. Communications in Mathematical Sciences, 3(2):133-157, 2005.

[3] M. Cada and M. Torrilhon. Compact third-order limiter functions for finite volume methods. Journal of Computational Physics, 228(11):4118-4145, 2009.

[4] A. F. Castelo, M. F. Tomé, C. N. L. César, S. McKee, and J. A. Culminato. Freeflow: An integrated simulation system for three-dimensional free-surface flows. Journal of Computers and Visualization in Science, 2:199-210, 2000.

[5] A. J. Chorin. Numerical solution of the Navier-Stokes equations. Mathematics Computational, 22:745-762, 1968.

[6] A. Colagrossi and M Landrini. Numerical simulation of interfacial flows by smoothed particle hydrodynamics. Journal of Computational Physics, 191(2):448-475, 2003.

[7] L. Corrêa. Um novo esquema upwind de alta resolução para equações de conservação não estacionárias dominadas por convecção. Dissertação de Mestrado, Universidade de São Paulo - Instituto de Ciências Matemáticas e de computação, 2011.

[8] J.O. Cruickshank. Low-Reynolds-number instabilities in stagnating jet flows. Journal of Fluid Mechanics, 193(1):111-127, 1988.

[9] C. Ellegaard, A.E. Hansen, A. Haaning, K. Hansen, A. Marcussen, T. Bohr, J.L. Hansen, and S. Watanabe. Cover illustration: Polygonal hydraulic jumps. Nonlinearity, 12:1, 1999.

[10] V.G. Ferreira, F.A. Kurokawa, R.A.B. Queiroz, M.K. Kaibara, C.M. Oishi, J.A. Cuminato, A. Castelo, M.F. Tomé, and S. Mckee. Assessment of a high-order finite difference 
upwind scheme for the simulation of convection-diffusion problems. International Journal for Numerical Methods in Fluids, 60:1-26, 2009.

[11] V.G. Ferreira, R.A.B. Queiroz, F.A. Kurokawa, R.G. Cuenca, C.M. Oishi, G. A. B. Lima, and S. Mckee. An upwind differencing scheme for conservation laws and related fluid dynamics problems. Submetido ao Journal of Computational Physics, 2009.

[12] P. H. Gaskell and A. K. C. Lau. Curvature-compensated convective transport: Smart, a new boundedness preserving transport algorithm. International Journal for Numerical Methods in Fluids, 8:617-641, 1988.

[13] F. H. Harlow and E. Welch. Numerical calculation of time-dependent viscous incompressible flow of fluid with free surface. The Physics of Fluids, 8:2182-2189, 1965.

[14] A. Harten. High resolution schemes for hyperbolic conservation laws. Journal of Computational Physics, 49:357-393, 1983.

[15] S. Jin and Z. Xin. The relaxation schemes for systems of conservation laws in arbitrary space dimensions. Communications on Pure and Applied Mathematics, 48(3):235-276, 1995.

[16] K. B. Kuan and C. A. Lin. Adaptive QUICK-Based scheme to approximate convective transport. AIAA Journal, 38(12):2233-2237, 2000.

[17] B. P. Leonard. The QUICK algorithm: a uniformly third-order finite difference method for highly convective flows. Computational Methods Applicate Mechanic Engineering, 19:59, 1979.

[18] B. P. Leonard. A stable and accurate convective modelling procedure based on quadratic interpolation. Computer Methods in Applied Mechanics and Engineering, 19:59-98, 1979.

[19] B. P. Leonard. Simple high-accuracy resolution program for convective modelling of discontinuities. International Journal for Numerical Methods in Fluids, 8:1291-1318, 1988.

[20] B.P. Leonard. Universal limiter for transient interpolation modeling of the advective transport equations: The ultimate conservative difference scheme. NASA Technical Memorandum, 100916, 1988.

[21] R. J. LeVeque. Finite volumes methods for hyperbolic problems. Press Syndicate of the University of Cambridge, 2004.

[22] C. H. Lin and C. A. Lint. Simple high-order bounded convection scheme to model discontinuities. AIAA Journal, 35(3):563-565, 1997. 
[23] H. Lin and C.C. Chieng. Characteristic-based flux limiters of an essentially third-order flux-splitting method for hyperbolic conservation laws. International Journal for Numerical Methods in Fluids, 13:287-307, 1991.

[24] P. J. Oliveira M. A. Alves and F. T. Pinho. A convergent and universally bounded interpolation schemes for the treatment of advection. International Journal for Numerical Methods in Fluids, 41:47-75, 2003.

[25] V. G. Ferreira M. F. Tomé, A. Castelo and S. McKee. A finite difference technique for solving the Oldroyd-b model for 3d-unsteady free surface flows. Journal Non-Newtonian Fluid Mechanics, 154:179-206, 2008.

[26] J.C. Mandal and J. Subramanian. On the link between weighted least-squares and limiters used in higher-order reconstructions for finite volume computations of hyperbolic equations. Applied Numerical Mathematics, 58(5):705-725, 2008.

[27] J.C. Martin and W.J. Moyce. Part IV. An experimental study of the collapse of liquid columns on a rigid horizontal plane. Philosophical Transactions of the Royal Society of London. Series A, Mathematical and Physical Sciences, 244(882):312, 1952.

[28] J.M. Nóbrega, O.S. Carneiro, F.T. Pinho, G.S. Paulo, M.F. Tomé, A. Castelo, and J.A. Cuminato. The phenomenon of jet buckling: Experimental results and numerical predictions. In The Polymer Processing Society 23rd Annual Meeting, 2007.

[29] C. M. Oishi, J. A. Cuminato, V. G. Ferreira, M. F. Tomé, A. Castelo, N. Mangiavacchi, and S. McKee. A Stable semi-implicit method for free surface flow. Trans. ASME Journal of Applied Mechanics, 73:940-947, 2006.

[30] M. B. Oliveira. Freeflow-axis: um ambiente de simulação de escoamentos axissimétricos com superfícies livres. Dissertação de Mestrado, Universidade de São Paulo - Instituto de Ciências Matemáticas e de computação, 2002.

[31] R. Peyret and T. D. Taylor. Computational methods for fluid flow. New York, 1983.

[32] J. Qiu and C.W Shu. Finite difference WENO schemes with Lax-Wendroff-type time discretizations. SIAM Journal on Scientific Computing, 24(6):2185-2200, 2003.

[33] R.A.B Queiroz. Desenvolvimento e teste de esquemas upwind de alta resolução e suas aplicações em escoamentos incompressíveis com superfícies livres. Dissertação de Mestrado, Universidade de São Paulo - Instituto de Ciências Matemáticas e de computação, 2009.

[34] A. Rai, B. S. Dandapat, and S Poria. Circular hydraulic jump in generalized-Newtonian fluids. ArXiv:0809.2231, 2008. 
[35] N.M. Ribe, E. Stutzmann, Y. Ren, and R. Van Der Hilst. Buckling instabilities of subducted lithosphere beneath the transition zone. Earth and Planetary Science Letters, 254(1-2):173-179, 2007.

[36] W.J.Rider, J.A. Greenough, and J.R. Kamm. Accurate monotonicity-and extrema-preserving methods through adaptive nonlinear hybridizations. Journal of Computational Physics, 225(2):1827-1848, 2007.

[37] V. G. Ferreira J. A. Cuminato A. Castelo F. S. Sousa S. McKee, M. F. Tomé and N. Mangiavacchi. The MAC method. Computers \& Fluids, 37:907-930, 2008.

[38] J. Shi, Y.T. Zhang, and C.W. Shu. Resolution of high order weno schemes for complicated flow structures. Journal of Computational Physics, 186(2):690-696, 2003.

[39] B. Song, G. R. Liu, K.Y. Lam, and R.S. Amano. On a higher-order bounded discretization scheme. International Journal for Numerical Methods in Fluids, 32(7):881-897, 2000.

[40] B. Liu, G. R. Lam, K. Y. Song and R. S. Amano. On a higer-order bounded discretization scheme. International Journal for Numerical Methods in Fluids, pages 32:881-887, 2000.

[41] P. K. Sweby. High resolution schemes using flux limiters for hyperbolic conservation laws. SIAM Journal on Numerical Analysis, 21:995-1011, 1884.

[42] S.G.I. Taylor, J. Friedman, J. Hirschfeld, Inc Educational Services, Encyclopaedia Britannica Educational Corporation, and National Committee for Fluid Mechanics Films. Low Reynolds number flows. Encyclopaedia Britannica Educational Corporation distributor, 1988.

[43] Zhen H. Teng. Exact boundary conditions for the initial value problem of convex conservation laws. Journal of Computational Physics, 229:3792-3801, 2010.

[44] M.F Tomé, J. A. Cuminato, N. Mangiavacchi, and S. McKee. GENSMAC3D: a numerical method for solving unsteady three-dimensional free surface flows. International Journal for Numerical Methods in Fluids, 37(7):747-796, 2001.

[45] E. F. Toro. Rieman solvers and numerical methods for fluids dynamics. New York, 1999.

[46] G.D. V. Albada, B. V. Leer, and J. W.W. Roberts. A comparative study of computational methods in cosmic gas dynamics. Astronomy and Astrophysics, 108:76-84, 1982.

[47] A. Varonos and G. Bergeles. Development and assessment of a variable-order non-oscillatory scheme for convection term discretization. International Journal for $\mathrm{Nu}$ merical Methods in Fluids, 26(1):1-16, 1998. 
[48] V. Venkatakrishnan. Preconditioned conjugate gradient methods for the compressible navier-stokes equations. In AIAA, Aerospace Sciences Meeting, volume 1, 1990.

[49] L. Ville, L. Silva, and T. Coupez. Convected level set method for the numerical simulation of fluid buckling. International Journal for Numerical Methods in Fluids, 66(3):324-344, 2011.

[50] N. P. Waterson and H. Deconinck. Design principles for bounded higher-order convection schemes - a unified approach. Journal of Computational Physics, 224:182-207, 2007.

[51] E. J Watson. The radial spread of a liquid jet over a horizontal plane. Journal of Fluid Mechanics, 20(03):481-499, 1964.

[52] G. W. Wei and Y. Gu. Conjugated filter approach for solving Burger's equation. Journal of Computational and Applied Mathematics, 149:439-456, 2002.

[53] P. Woodward and P. Colella. The numerical simulation of two-dimensional fluid flow with strong shocks. Journal of Computational Physics, 54(1):115-173, 1984.

[54] S.C. Xue, N. Phan-Thien, and R.I Tanner. Upwinding with deferred correction (UPDC): an effective implementation of higher-order convection schemes for implicit finite volume methods. Journal of Non-Newtonian Fluid Mechanics, 108(1-3):1-24, 2002.

[55] J. Zhu. On the higher-order bounded discretization schemes for finite volume computations of incompressive flows. In Computer Methods in Applied Mechanics and Engineering,345-360. 